\title{
Paradoxical Roles of Nanoparticles in Cancer Therapeutics and Carcinogenesis
}

\author{
Emily Despeaux
}

Follow this and additional works at: https://researchrepository.wvu.edu/etd

\section{Recommended Citation}

Despeaux, Emily, "Paradoxical Roles of Nanoparticles in Cancer Therapeutics and Carcinogenesis" (2016). Graduate Theses, Dissertations, and Problem Reports. 7080.

https://researchrepository.wvu.edu/etd/7080

This Dissertation is protected by copyright and/or related rights. It has been brought to you by the The Research Repository @ WVU with permission from the rights-holder(s). You are free to use this Dissertation in any way that is permitted by the copyright and related rights legislation that applies to your use. For other uses you must obtain permission from the rights-holder(s) directly, unless additional rights are indicated by a Creative Commons license in the record and/ or on the work itself. This Dissertation has been accepted for inclusion in WVU Graduate Theses, Dissertations, and Problem Reports collection by an authorized administrator of The Research Repository @ WVU.

For more information, please contact researchrepository@mail.wvu.edu. 


\title{
Paradoxical Roles of Nanoparticles in Cancer Therapeutics and Carcinogenesis
}

\author{
Emily Despeaux
}

Dissertation submitted to the School of Pharmacy at West Virginia University in partial fulfillment of the requirements for the degree

\section{Doctor of Philosophy}

\section{Pharmaceutical and Pharmacological Sciences}

\author{
Yon Rojanasakul, PhD, Chair \\ Vincent Castranova, PhD, Chair \\ Mohindar Seehra, PhD \\ Raymond Raylman, PhD \\ William Petros, Pharm.D \\ Liying Wang, PhD \\ School of Pharmacy \\ Morgantown, West Virginia \\ 2016
}

Keywords: Nanotoxicity, Nanotherapeutics, SPION, CNT, survivin, mesothelin 


\section{Abstract}

\section{Paradoxical Roles of Nanoparticles in Cancer Therapeutics and Carcinogenesis}

\section{Emily Despeaux}

Nanoparticles (NPs) are becoming increasingly common in consumer goods and are under investigation for a variety of industrial and biomedical applications. However, challenges in determining NP toxicity may prevent them from reaching their full potential. NPs cannot be treated as single class for toxicity evaluations. Even among particles made from the same material, particle-specific physical properties, including size, shape, surface charge, agglomeration state, and surface modifications have a strong effect on the toxicity. Even so, the obstacles to conclusively and reproducibly evaluating toxicity span all NP classes. NP literature is riddled with confusing and often contradictory reports regarding the biocompatibility of both engineered NPs, designed with biocompatibility as a priority, and NPs from occupational or environmental exposures. Incomplete NP characterization and sample inhomogeneity represent major confounding factors in disparate results from seemingly comparable study setups. Additionally, NPs can interfere with many conventional toxicity screening methods. Inappropriate doses, exposure routes, and toxicity endpoints further diminish the utility of many published studies.

Given the burgeoning interest in NP-based therapeutic agents, consistent, reliable standards are needed to ensure the biocompatibility of new formulations. To those ends, the synthesis, characterization, and in vitro toxicity of a multi-functional NP therapeutic were investigated (Chapter 2). Specifically, superparamagnetic iron oxide nanoparticles (SPIONs) were coated with amphiphilic polymer and functionalized with antisense oligonucleotides targeting survivin, an anti-apoptotic protein that is highly overexpressed in cancer. SPION physical properties, including particle size and composition, were characterized at each step of synthesis. Our results showed that the SPION platform is biocompatible and capable of delivering functional antisense oligonucleotides to regulate survivin expression; however, significant refinement of the DNA-to-SPION coupling step is needed. Applied clinically, antisense survivin coupled SPIONs can reduce the required dose of, adverse effects from, and resistance to, current cancer chemotherapy regimens.

In contrast to engineered NPs for biomedical applications, where real-world exposures would involve careful control of both exposure time- and dose, occupational NP exposures are variable, chronic, and difficult to model in laboratory settings. Chapter 3 focuses on identifying the mechanisms behind carbon nanotube (CNT)-induced malignant transformation of bronchial epithelial cells using a chronic in vitro exposure model. We specifically investigated the role of mesothelin (MSLN), a cell-surface protein that is highly overexpressed in many cancers, in the aggressive phenotype noted following chronic, low-dose CNT exposure. MSLN knockdown resulted in significantly 
decreased invasion, migration, colonies on soft agar, and tumor sphere formation. In vivo, MSLN knockdown cells formed smaller primary tumors and less metastases. The mechanism by which MSLN contributes to these more aggressive behaviors was investigated using Ingenuity Pathway Analysis, which predicted that increased MSLN could induce cyclin $\mathrm{E}$, a cell cycle regulator known to be associated with human cancer. We found that MSLN knockdown cells had decreased cyclin E, and their proliferation rate was reverted to nearly that of untransformed cells. Cell cycle analysis results were consistent with the decreased rate of proliferation. Together, our results indicate a novel role of MSLN in the malignant transformation of bronchial epithelial cells following CNT exposure, suggesting its utility as a potential biomarker and drug target for CNT-induced malignancies.

As demonstrated by the two studies presented here, NPs have the potential to function as both cancer therapeutics and carcinogens. Careful evaluation of toxicity, ensuring that appropriate doses, assays, exposure routes, and endpoints are used, is imperative. Elucidating the physical properties and functionalization that contribute to toxicity, and the mechanisms of that toxicity, will allow NP benefits to be fully exploited while minimizing the risk of widespread, detrimental public health effects 


\section{Acknowledgements}

First and foremost, I would like to thank my advisors, Dr. Yon Rojanasakul and Dr. Vince Castranova, who welcomed me into their group on short notice. I greatly appreciate the opportunities they provided and their efforts towards my timely graduation. I would also like to thank my committee members, with a special thanks to those members who have been part of this project since its inception and have continued to offer guidance as the project evolved. Dr. David Siderovski and Dr. Kimberly Quedado have been invaluable resources and I am grateful for the time and effort they have put into ensuring my success.

I would like to thank Kelly Pisane and Dr. Mohindar Seehra, whose collaborations provided much needed characterization data. Dr. Sierra Talbot was immensely helpful, and her thoughtful responses and discussions helped shape the early stages of this project. I would also like to thank the members of Dr. Rojanasakul's lab, particularly Katie He and Maria Voronkova, for their assistance when I joined their lab.

The members of my former lab, including Chris Bostick and Katherine Hickey, contributed greatly to my success during my time at WVU. Reem Eldawud has also been a great help. I would also like to thank Penny Dailey for her help in navigating through the turmoil over the last few years.

Lastly, I would like to thank my family for their ongoing support. 


\section{Table of Contents}

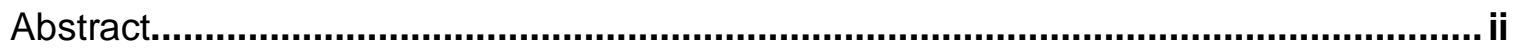

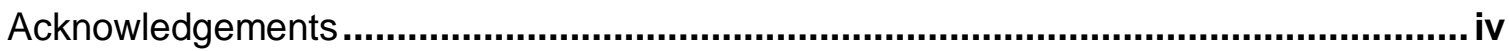

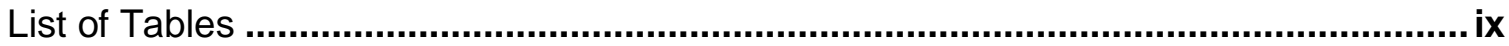

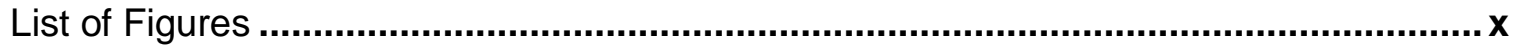

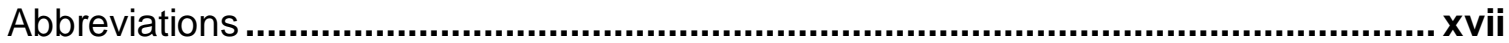

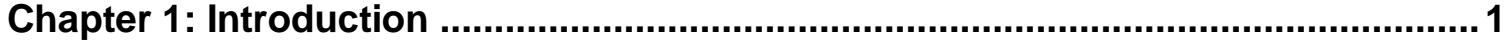

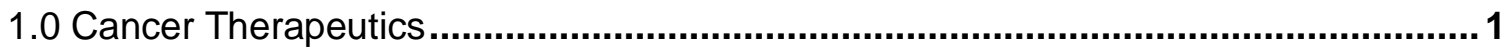

1.0.1 Cancer Development..................................................................................................................................1

1.0.2 Conventional Cancer Therapeutics .................................................................................................................2

1.0.3 Targeted Cancer Therapies .....................................................................................................................................

1.0.3a Pharmaceutical versus Physical Targeting .....................................................................................4

1.0.3b Strategies for Targeting Tumors..........................................................................................................

1.0.4 Antisense Oligonucleotides as Cancer Therapeutics.............................................................................7

1.0.4a Regulation of Protein Expression with Antisense Oligonucleotides .........................................

1.0.4b Delivery of Antisense Agents in vitro.....................................................................................................

1.0.4c Delivery of Antisense Agents in vivo..................................................................................................

1.0.4d Clinically Relevant ASO.........................................................................................................................10

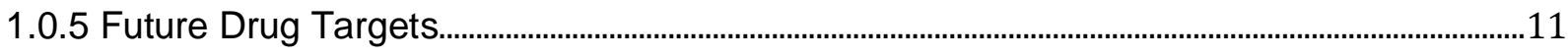

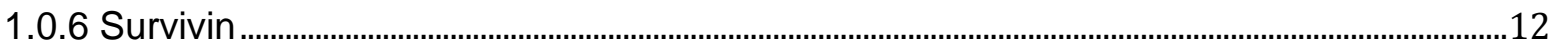

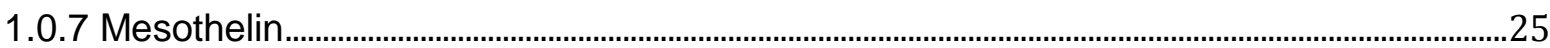

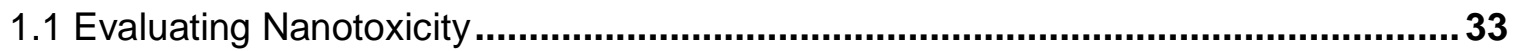

1.1.1 Introduction to Nanoparticles............................................................................................................33

1.1.2 Nanoparticle Toxicity ...........................................................................................................................

1.1.3 Role of NP Physical Properties............................................................................................................35

1.1.4 In Vitro Toxicity Assessment ...................................................................................................36

1.1.5 In Vivo Toxicity Assessment..............................................................................................................37

1.1.6 Dosing NPs for Toxicity Evaluations......................................................................................37

1.1.7 Dosing Considerations: NP Quantification \& Dosing Metrics......................................................38

1.1.8 Dosing Considerations: Modeling Real World Exposures ..........................................................4

1.1.9 Strategies for Evaluating NP Toxicity .............................................................................................4

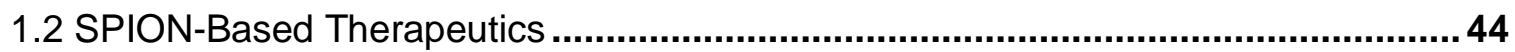

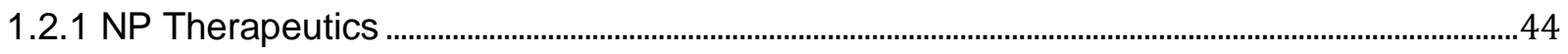




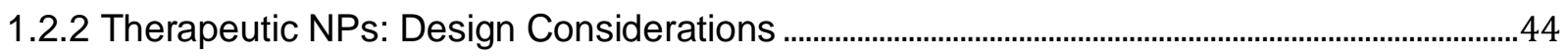

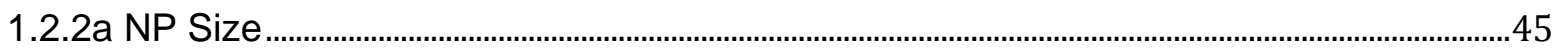

1.2.2b Minimizing Plasma Protein Adsorption............................................................................................4

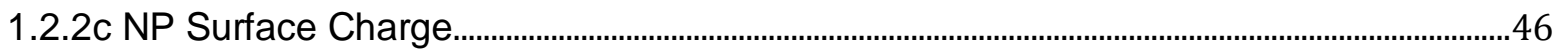

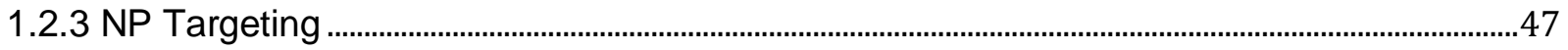

1.2.4 NP Surface Modification Strategies .....................................................................................................4

1.2.5 SPIONs as Multi-Functional Therapeutics..................................................................................................51

1.2.5a SPION Therapeutics..................................................................................................................................52

1.2.5b SPIONs as MRI Contrast Agents..........................................................................................................53

1.2.5c SPIONs as Drug Delivery Vehicles.......................................................................................................54

1.2.5d Multi-functional SPION Systems ......................................................................................................56

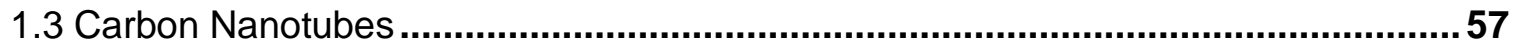

1.3.1 Carbon Nanotubes...............................................................................................................................................

1.3.2 Carbon Nanotube Toxicity ...............................................................................................................................57

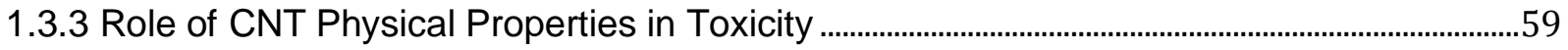

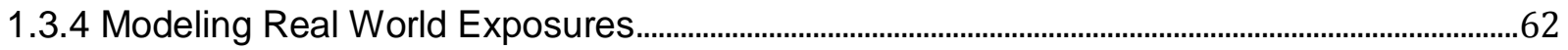

1.3.5 Acute Toxicity of CNTs..................................................................................................................................64

1.3.6 Chronic Toxicity of CNTs ..........................................................................................................................64

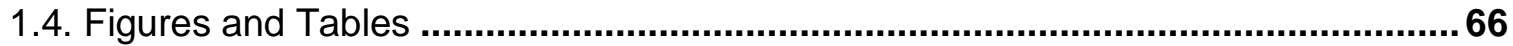

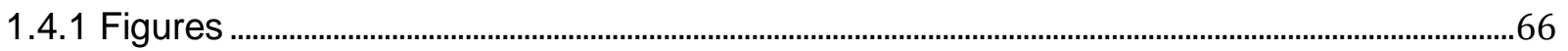

1.4.2 Tables .........................................................................................................................................................69

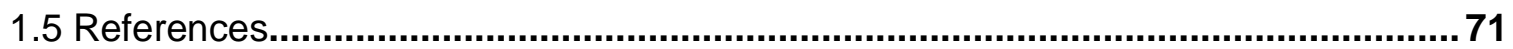

Chapter 2: Targeting Survivin: Antisense-coupled SPIONs for Targeted Cancer Imaging and Therapy..........................................................................................................112

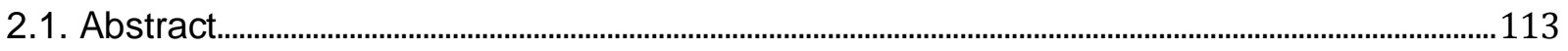

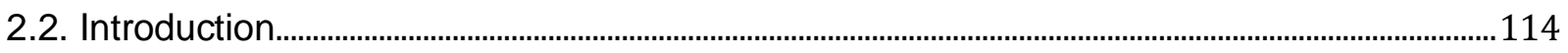

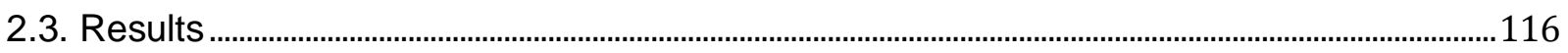

2.3.1. SPION Synthesis and Characterization...............................................................................116

2.3.2. Coupling DNA to SPION-PAA......................................................................................119

2.3.3. SASO-SPION Decrease Survivin Expression and Reduce Survival in A549 Cells, a High Survivin Human Lung Cancer Cell Line.........................................................................................120

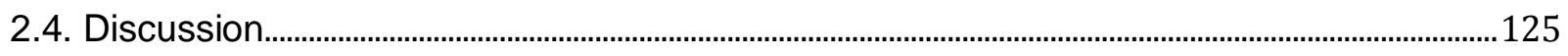

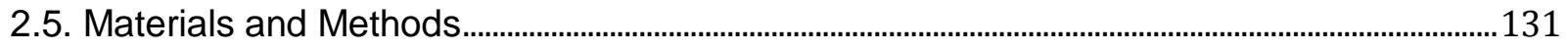

2.5.1 SPION Synthesis..........................................................................................................................1131

2.5.2 Octylamine-modified Polyacrylic Acid (PAA) Synthesis ......................................................131 
2.5.3 Oligonucleotide Synthesis and Purification .................................................................................132

2.5.4 Oligonucleotide-SPION Coupling...........................................................................................133

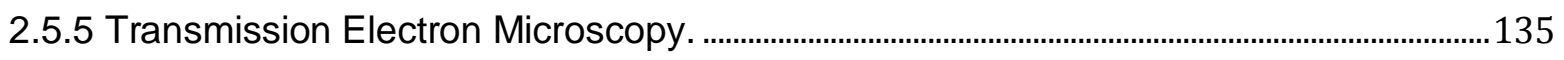

2.5.6 Dynamic Light Scattering...........................................................................................................135

2.5.7 Thermogravimetric Analysis ......................................................................................................136

2.5.8 Inductively Coupled Plasma (ICP) ........................................................................................136

2.5.9 General Cell Culture ........................................................................................................................136

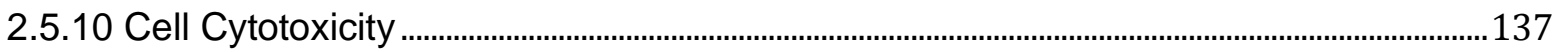

2.5.11 Cell Metabolic Viability .....................................................................................................................138

2.5.12 Western Blots....................................................................................................................................139

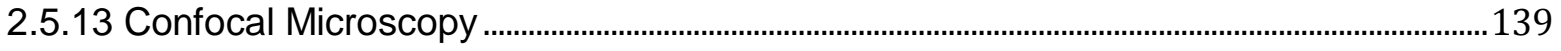

2.5.14 Fluorescent Microscopy..................................................................................................................140

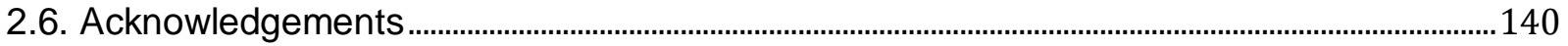

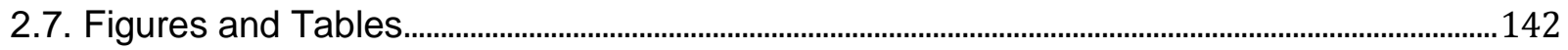

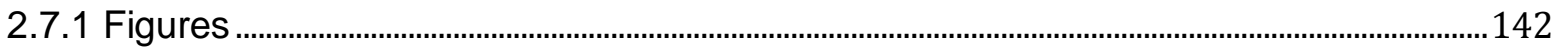

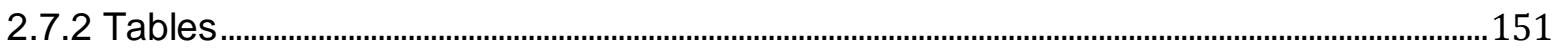

2.8. References .......................................................................................................................................152

Chapter 3: Role of Mesothelin in Carbon Nanotube-Induced Carcinogenic Transformation of Human Bronchial Epithelial Cells ..................................................................... 164

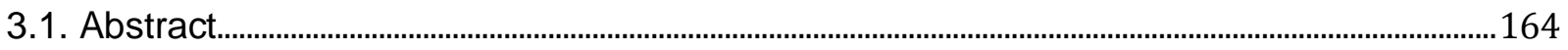

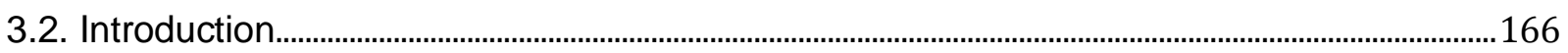

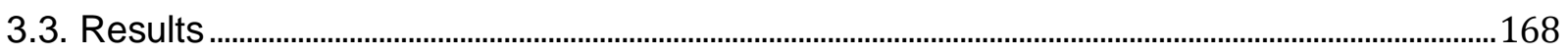

2.3.1. Mesothelin expression is increased in human lung tumors and lung cancer cell lines 168

2.3.2. MSLN knockdown BSW cells are less aggressive than BSW cells in vitro and in vivo 169

2.3.3. Mesothelin increases cyclin E, which drives more rapid cell proliferation.......................172

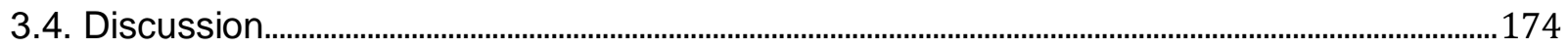

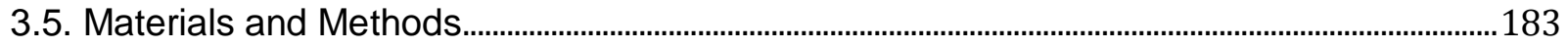

3.5.1 Patient tumor samples .....................................................................................................................183

3.5.2 Cell Culture..............................................................................................................................................183

3.5.3 Generation of stable MSLN knockdown cells .........................................................................184

3.5.4 Transient MSLN overexpression in B2B cells. .......................................................................184

3.5.5 Cell proliferation ........................................................................................................................184

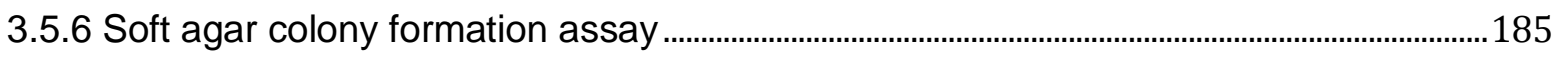




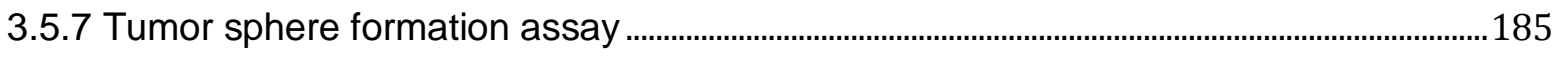

3.5.8 Cell migration and invasion assays. .......................................................................................185

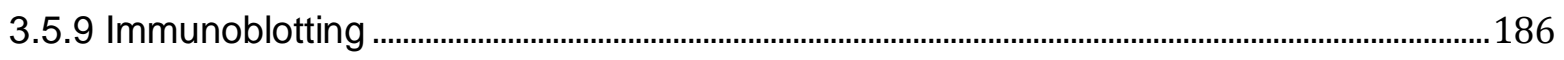

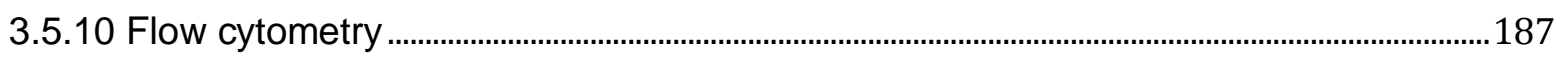

3.5.11 Tumor xenograft mouse models.....................................................................................................187

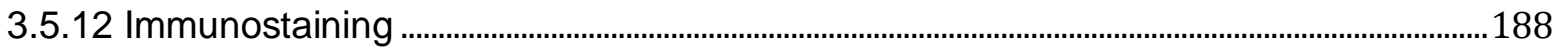

3.5.13 Ingenuity Pathway Analysis.........................................................................................................189

3.5.14 Statistics ......................................................................................................................................189

3.6. Acknowledgements ........................................................................................................................190

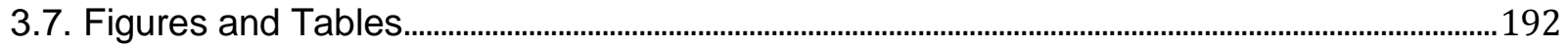

Figure 3.4. MSLN is overexpressed in lung cancer...........................................................................192

Figure 3.5. Knockdown MSLN reduces soft agar colony and tumor sphere formation........193

Figure 3.6. MSLN expression increases migration and invasion in vitro......................................194

Figure 3.7. MSLN regulates tumor growth and metastasis in vivo..............................................195

Figure 3.8. Changes in gene expression when MSLN is increased in BSW cells. ................196

Figure 3.9. MSLN regulates cyclin $E$ and cell proliferation. .................................................................197

Figure 3.10. Cell cycle progression is affected by MSLN. ..............................................................198

3.8. References ...........................................................................................................................................199

Chapter 4: Project Insights and Future Directions ................................................ 208

4.1 Design and Evaluation of Multi-functional SPION Platform.................................................................208

4.1.1 Optimization of SPION-DNA Coupling Procedure..................................................................209

4.1.2 Characterization of Functionalized SPION platform ................................................................215

4.1.3 Evaluation of Biological Effects of SPION-ASO ......................................................................224

4.1.4 Selection of a Targeting Ligand to Improve the Selectivity of SPION Uptake by Survivin-Expressing Cancer Cells ......................................................................................................233

4.1.4b Selection of an aptamer targeting external feature of survivin expressing cells........237

4.2 Role of MSLN in CNT-induced Malignant Transformation ..............................................................239

4.2.1 MSLN-Related Changes in Gene Expression of CNT-transformed Cells........................240

4.2.2 MSLN as a Survivin Regulator ..................................................................................................24

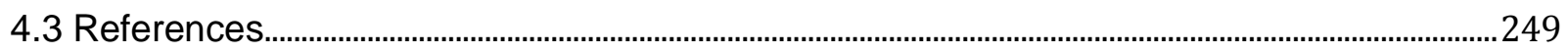




\section{List of Tables}

\section{Chapter 1. Introduction}

Table 1. Survivin expression in human tumor samples. ..................................69

Table 2. Interference of NPs with in vitro assessments of toxicity .................... 70

Chapter 2. Targeting Survivin: Antisense-coupled SPIONs for Targeted Cancer Imaging and Therapy

Table 2.1. Contribution of various SPION layers to total particle diameter. Particle diameters were determined using DLS. Expected changes were calculated based on a single layer of covalently bound coating for both oleic acid and DNA. 151 


\section{List of Figures}

\section{Chapter 1.}

Figure 1. Specificity of NPs, in the form of tissue specific effects, can be conferred through two distinct mechanisms. (A) NPs that target a cell- or tissue specific pathway will be taken up by all cells but only affect cells that have the target feature. For the purposes of demonstration, the illustration shows NPs bearing antisense oligonucleotides that target a cancer specific protein. (B) NPs that recognize surface features of target cells will be internalized with greater specificity. Antibodies targeting cell surface features can be used to improve uptake specificity, as can molecules that are readily internalized,

including folate. 66

Figure 2. Mechanism of ASO protein synthesis inhibition. Binding of the ASO to the complementary sequence of the target protein mRNA occurs in the cytoplasm. Ribosomal translocation along the mRNA is physically blocked by ASO hybridization with its complementary sequence. 67

Figure 3. Structure of YM155 (1-(2-Methoxyethyl)-2-methyl-4,9-dioxo-3-(pyrazin-2ylmethyl)-4,9-dihydro-1 $H$-naphtho[2,3-d] imidazolium bromide), a small molecule inhibitor of survivin. 68 


\section{Chapter 2. Targeting Survivin: Antisense-coupled SPIONs for Targeted Cancer Imaging and Therapy}

Figure 2.1. Schematic of SPION platform. (Left) SPION-OA is synthesized via thermal decomposition synthesis, (center) coated with octylamine modified PAA, and (left) aminemodified DNA is coupled to the outward-facing carboxylic acid groups of the PAA coating. The hydrophobic layer formed by alkyl tails of the oleic acid and octylamine could be loaded with lipophilic drugs (red dots). 142

Figure 2.2. (A) Representative TEM image of SPION-OA. SPIONs are well dispersed and have uniform iron oxide cores. (B) SPION-OA iron oxide core diameter, measured across three separately prepared syntheses, is $7.05 \pm 0.08 \mathrm{~nm}$. 143

Figure 2.3. (A) FT-IR spectra for SPION-OA and (B) SPION-PAA. Analysis was performed on desiccator-dried powder samples. 144

Figure 2.4. A) Representative TGA of SPION-OA and (B) SPION-PAA. Red line shows percent of original mass remaining as temperature was increased $\left(5^{\circ} \mathrm{C} /\right.$ minute $)$. Black line is the derivative of the red line, showing the rate of change of the mass as temperature was increased. (C) Graphical representation of the composition of SPION-OA and SPION-PAA. Composition was determined by TGA and was based on the analysis of three samples from three separate batches. (D) Hydrodynamic diameter of SPION-OA in toluene and (E) SPION-PAA in distilled water. 145 
Figure 2.5. (A) Representative TEM image of SASO-SPION. SASO-SPION is well dispersed and has uniform iron oxide cores. (B) Graphical comparison of iron oxide core diameter of a single batch of SPION-OA $(7.07 \pm 0.74 \mathrm{~nm})$ and SASO-SPION $(7.07 \pm 0.74$ nm) prepared from that batch. (C) Hydrodynamic diameter of SASO-SPION in distilled water, as determined by DLS, is $50.58 \mathrm{~nm}$ 146

Figure 2.6. To-scale diagram of contribution of various SPION layers to hydrodynamic diameter (determined by DLS). 147

Figure 2.7. SASO-SPION is taken up by A549 cells following 24 hour incubation. Cells were incubated with SASO-SPION or fluorescein- SASO-SPION $(0,20,200 \mu \mathrm{g} / \mathrm{mL})$ for 24 hours, washed to remove free SPIONs, and stained with FM4-64X cell membrane stain before fixation. Fluorescein- SASO-SPION is visible inside the cells, particularly at the high $(200 \mu \mathrm{g} / \mathrm{mL})$ dose, as shown in the insets. 148

Figure 2.8. (A) Fluorescently-labeled SASO-SPION is taken up by A549 cells following a 24 hour incubation. Cells were incubated with SASO-SPION or fluorescein-SASO-SPION (200 $\mu \mathrm{g} / \mathrm{mL})$ for 24 hours, washed to remove free SPIONs, and stained with FM4-64X cell membrane stain before fixation.3D reconstruction from confocal microscopy z-stack images shows that fluorescein-SASO-SPIONs (green) are inside of the FM4-64X stained cells (red). (B) Fluorescent microscopy shows co-localization (yellow) of fluoresceintagged SASO-SPION (green) and endosomes (red) after staining with Lysotracker Deep Red (left) or Cell Mask Orange (right). Cells were incubated with fluorescein-labeled 
SASO-SPION (33 $\mu \mathrm{g} \mathrm{Fe} / \mathrm{mL}$ ) for 24 hours, washed to remove free SPIONs, and stained with either Lysotracker Deep Red or Cell Mask Orange cell membrane stain before fixation. 149

Figure 2.9. (A) Survivin expression in A549 cells following 24 hour incubation. Results from 2 trials. (B) Metabolic viability, determined by WST-1 assay, of A549 cells was assessed after 24 hour incubation with SASO-SPION or NTC-SPION (0, 50, 100, 200, $500,1000 \mathrm{nM}$ DNA). Results are expressed as a percentage of the untreated control viability. Results of 4 independent trials. No significance $(p<0.05$, by Student's t-test) was noted. (C, D) Metabolic viability, determined by WST-1 assay, of A549 cells was assessed after 48 hour incubation with SASO-SPION or NTC-SPION $(0,50,100,200$, $500,1000 \mathrm{nM}$ DNA). Results are expressed as a percentage of the untreated control viability. (C) and (D) show the variation in biological effects resulting from inconsistencies in the DNA coupling process, despite treating cells with the same DNA concentration. 


\section{Chapter 3. Role of Mesothelin in Carbon Nanotube-Induced}

Carcinogenic Transformation of Human Bronchial Epithelial Cells

Figure 3.1. MSLN is overexpressed in lung cancer. (A) Western blot for MSLN in pairs of lung tumor lysates $(\mathrm{T})$ and normal tissue controls $(\mathrm{N})$ from the same patients. Pairs 16 are large cell carcinomas, pairs 7-9 are squamous cell carcinomas, and pair 10 is adenocarcinoma. (B) Quantification of tumor MSLN expression, relative to actin. (C) Western blot and (D) quantification of MSLN in non-cancerous bronchial epithelial cells (BEAS-2B), CNT-transformed BSW cells, and in two established lung cancer cell lines (A549 and $\mathrm{H} 460) .{ }^{*} \mathrm{P}<0.05$ vs. BEAS-2B 192

Figure 3.2. Knockdown MSLN reduces soft agar colony and tumor sphere formation. (A) Western blot and (B) quantification of MSLN in several stable knockdown clones. (C) Representative images showing colony formation on soft agar of BSW shC and BSW shMSLN cells. (D) Representative images of tumor sphere formation of BSW shC and BSW shMSLN cells. (E) Quantification of colonies formed by BSW shC and BSW shMSLN cells. (F) Quantification of tumor spheres formed by BSW shC and BSW shMSLN cells. ${ }^{*} P<0.05$ vs. BSW shC. (G) Western blot of MSLN expression in B2B and B2B/MSLN cells. $(\mathrm{H})$ Quantification and (I) representative images of colonies formed by B2B and B2B/MSLN cells. ${ }^{*} \mathrm{P}<0.05$ vs. B2B. 193

Figure 3.3. MSLN expression increases migration and invasion in vitro. (A) Representative images of BSW shC and BSW shMSLN migration and (B) invasion, stained with Diff-Quik. (C) Quantification of migrating BSW shC and BSW shMSLN cells. 
(D) Quantification of invading BSW shC and BSW shMSLN cells. ${ }^{*} P<0.05$ vs. BSW shC.

(E) Representative images of $B 2 B$ and $B 2 B / M S L N$ migration and $(F)$ invasion, stained with Diff-Quik. (G) Quantification of migrating B2B and B2B/MSLN cells. (H) Quantification of invading B2B and B2B/MSLN cells. ${ }^{*} \mathrm{P}<0.05$ vs. B2B 194

Figure 3.4. MSLN regulates tumor growth and metastasis in vivo. (A) Injection site tumors. (B) Graphical representation of injection-site tumor weights. (C) Number of thoracic and abdominal surface metastatic nodules. (D) Surface area of hepatic and pulmonary metastases. (E) Representative pulmonary and hepatic tissues with hematoxylin and eosin (H\&E) staining. Arrows denote metastatic tumor nodules. (F) Immunostaining of human mitochondria and $(G)$ human MSLN in representative liver and lung sections of mice injected with BSW shC or BSW shMSLN. Arrows denote metastatic tumor nodules. ${ }^{*} \mathrm{P}<0.05$ vs. BSW shC. 195

Figure 3.5. Changes in gene expression when MSLN is increased in BSW cells. Ingenuity Pathway Analysis (IPA) was used to predict differentially expressed genes. Yellow to orange colors represent up-regulation, while blue represent down-regulation, compared to BEAS-2B passage-matched controls. Color intensity signifies fold change. 196

Figure 3.6. MSLN regulates cyclin E and cell proliferation. (A) Western blot and (B) quantification of cyclin E in BSW shC and two MSLN knockdown clones. (C) Proliferation of BSW shC and BSW shMSLN cells, determined using Promega Aqueous One-Step 
viability assay, which measures mitochondrial metabolic rate, at 24 and 48 hours after seeding. ${ }^{*} \mathrm{P}<0.05$ vs. BSW shC

Figure 3.7. Cell cycle progression is affected by MSLN. (A) Representative flow cytometry plot for propidium iodide stained BSW shC and (B) BSW shMSLN cells. (C) Graphical representation of cell cycle distribution, determined from flow cytometry. FACS Express 5 software was used for analysis and proliferation statistics (green and red traces). ${ }^{*} \mathrm{P}<0.05$ vs. BSW shC. 198 


\section{Abbreviations}

$\begin{array}{ll}\mu & \text { micro } \\ \text { A } & \text { Adenosine } \\ \text { A549 } & \text { Immortalized human lung adenocarcinoma cells } \\ \text { AKT } & \text { Protein kinase B } \\ \text { ASO } & \text { Antisense oligonucleotide } \\ \text { BAD } & \text { Pro-apoptotic mediator } \\ \text { BAX } & \text { Pro-apoptotic mediator } \\ \text { BCl-2 } & \text { B-cell lymphoma 2 (anti-apoptotic mediator) } \\ \text { BEAS-2B } & \text { Immortalized human bronchial epithelial cells } \\ \text { BSA } & \text { Bovine serum albumin } \\ \text { BSW } & \text { Single-wall carbon nanotube transformed bronchial epithelial cells } \\ \text { C } & \text { Cytosine } \\ \text { CA125 } & \text { Cancer antigen 125 (also known as MUC16) } \\ \text { CDK } & \text { Cyclin-dependent kinase } \\ \text { CisR } & \text { Cisplatin resistant } \\ \text { CNT } & \text { Carbon nanotube } \\ \text { DLS } & \text { Dynamic light scattering } \\ \text { DMEM } & \text { Dulbecco's modified Eagle medium } \\ \text { DMF } & \text { Dimethylformamide } \\ \text { DMSO } & \text { Dimethyl sulfoxide } \\ \text { DMTMM } & \text { 4-(4,6-dimethody-1,3,5-triazin-2-yl)-4-methylmorpholinium chloride } \\ \text { DNA } & \text { Deoxyribonucleic acid } \\ \text { ECL } & \text { Enhanced chemiluminescence } \\ \text { EDC } & \text { 1-Ethyl-3-(3-dimethylaminopropyl)carbodiimide } \\ \text { EGFR } & \text { Epidermal growth factor receptor } \\ \text { FBS } & \text { Fetal bovine serum } \\ \text { FDA } & \text { Food and Drug Administration } \\ \text { FT-IR } & \text { Fourier transformed infrared spectroscopy } \\ \text { g } & \text { Acceleration due to gravity } \\ \text { G } & \text { Guanine } \\ \text { GPI } & \text { Glycosylphosphatidylinositol } \\ \text { H460 } & \text { Immortalized human large cell carcinoma cells } \\ \text { HER2 } & \text { Human epidermal growth factor receptor 2 } \\ \text { HIF-1 } & \text { Hypoxia inducible factor-1 } \\ \text { HLA } & \text { Human leukocyte antigen } \\ \text { HRP } & \text { Horseradish peroxidase } \\ \text { IAP } & \text { Inhibitor of apoptosis proteins } \\ \text { ICP-OES } & \text { Inductively coupled plasma optical emission spectrometry } \\ \text { IL } & \text { Interleukin } \\ \text { IPA } & \text { Ingenuity Pathway Analysis } \\ \text { IV } & \text { Intravenous } \\ \text { kDa } & \text { Kilo Dalton } \\ \text { LDH } & \text { Lactate dehydrogenase } \\ & \end{array}$




\begin{tabular}{ll} 
M & Molar \\
m & Meter \\
Mcl-1 & Induced myeloid leukemia cell differentiation protein \\
MES & 2-(N-morpholino)ethanesulfonic acid (buffer) \\
Met5A & Immortalized human mesothelioma cell line \\
MHC & Major histocompatibility complex \\
MMP & Matrix metalloproteinase \\
mol & Mole \\
MRI & Magnetic resonance imaging \\
mRNA & Messenger ribonucleic acid \\
MSLN & Mesothelin \\
MTS & 3-(4,5-dimethylthiazol-2-yl)-5-(3-carboxymethoxyphenyl)-2-(4-sulfophenyl)-2H- \\
& tetrazolium \\
MTT & 3-(4,5-Dimethylthiazol-2-yl)-2,5-Diphenyltetrazolium Bromide \\
MUC16 & Mucin 16 (also known as CA125) \\
MWCNT & Multi wall carbon nanotube \\
MWCO & Molecular weight cutoff \\
n & nano \\
NF-KB & Nuclear factor kappa-light-chain-enhancer of activated B cells \\
NIH & National Institutes of Health \\
NP & Nanoparticle \\
NSCLC & Non-small cell lung cancer \\
NTC & Non-targeting control oligonucleotide \\
OA & Oleic acid \\
OD & Optical density \\
PAA & Poly(acrylic acid) \\
PEG & polyethylene glycol \\
PI & Propidium iodide \\
PI3K & Phosphatidylinositol-3-Kinase \\
pt & Parental (cell line) \\
PVDF & Polyvinylidene difluoride \\
RNA & Ribonucleic acid \\
ROS & Reactive oxygen species \\
RPMI-1640 & Roswell Park Memorial Institute-1640 medium \\
SAEC & Small airway epithelial cells \\
SASO & Survivin antisense oligonucleotide \\
SDS & Sodium dodecyl sulfate \\
shRNA & Short-hairpin ribonucleic acid \\
siRNA & Small interfering ribonucleic acid \\
SMRP & Soluble mesothelin related protein \\
SPION & Superparamagnetic iron oxide nanoparticle \\
ssDNA & Single stranded deoxyribonucleic acid \\
Stat3 & Signal transducer and activator of transcription 3 \\
SWCNT & Single-wall carbon nanotube \\
T & Thymine \\
& a \\
\hline
\end{tabular}




$\begin{array}{ll}\text { TBST } & \text { Tris-buffered saline } \\ \text { TEM } & \text { Transmission electron micscrope } \\ \text { TGA } & \text { Thermogravimetric analysis } \\ \text { TNF } & \text { Tumor necrosis factor } \\ \text { WST-1 } & \text { Water soluble tetrazolium } \\ \lambda & \text { Wavelength }\end{array}$




\section{Chapter 1: Introduction}

\subsection{Cancer Therapeutics}

\subsubsection{Cancer Development}

Cancer develops as a result of the accumulation of multiple damaging mutations to critical cell regulatory mechanisms. ${ }^{1}$ These changes typically occur in a step-wise fashion, with initial damage overriding either cell death pathways or cell replication damage checkpoints. Normally cells have a number of mechanisms in place to avoid propagating damaging genetic errors. During replication there are a number of checkpoints to ensure that only complete sets of intact genetic material are passed on and, if irreparable damage is present, the replication process is halted. Similarly, cells exposed to toxic substances, viral infections, or other damaging stimuli will often undergo apoptosis to prevent spreading the problem. Escaping these normal control mechanisms allows the damaged cell to replicate, and the propagation of these regulatory failures results allows mutations to accumulate.

Although each cancer develops its own unique collection of mutations and aberrant behaviors, there are several characteristic features of malignancies. ${ }^{1,2}$ Cancerous cells tend to proliferate faster than normal cells, even in the absence of normal growth signals. They are also resistant to normal apoptotic stimuli, including chemotherapy drugs or radiation, and their drug resistance generally increases throughout the course of treatment due to selective pressure. Resistance to physiologic apoptotic stimuli allows the malignant cells to continue to thrive even if they detach from their biological matrix, 
leading to circulating tumor cells and metastases. ${ }^{3}$ Additionally, rapidly growing tumors can stimulate angiogenesis to ensure a supply of oxygen and nutrients to support their increased metabolism.

Traditional cancer therapies attempt to exploit the characteristic behaviors of cancer cells to kill the malignant cells at a greater rate than healthy cells. Many chemotherapeutic agents interfere with cellular replication and metabolism. Drugs aiming to inhibit tumor blood supply have also been developed. While these traditional therapies have greatly improved cancer outcomes, they have a number of dose-limiting side effects because the pathways they target are also present in normal cells. Improved targeting of cancer therapies, where the drugs are able to accumulate in or mechanistically act only on tumor cells, is expected to significantly increase drug efficacy while reducing off-target effects.

\subsubsection{Conventional Cancer Therapeutics}

Conventional cancer therapeutics interfere with cellular metabolism and replication. Cancer cells grow and divide faster than healthy cells, meaning drugs targeting cellular metabolic and reproductive functions should preferentially effect the cancer cells. However, this preferential effect is minimal because these drugs act on pathways that are present and functioning in all cells. These indiscriminate mechanisms of destruction and systemic drug administration make severe side effects an inevitable consequence of these conventional treatments. Cells whose physiological functions require rapid turnover, like hair follicles, bone marrow, and the lining of the Gl tract, sustain the most damage from the off-target effects. This damage results in the side effects most 
commonly associated with chemotherapy: hair loss, anemia, weakened immune system, ${ }^{4}$ nausea, and vomiting. These ailments develop rapidly after treatment is initiated and are frequently accompanied by fatigue,,$^{5}$ impaired cognition,,$^{6-8}$ and loss of appetite. ${ }^{9}$ Cardiovascular ${ }^{10}$ and reproductive system dysfunction often follow. Thus, conventional treatment strategies require clinicians to walk a fine line between killing the cancer and killing the patient.

In addition to posing a direct threat to patients' health, ${ }^{11}$ the indirect effects on patient quality of life and mental state can prolong recovery. Weight loss in cancer patients is associated with decreased survival, but lack of appetite and Gl distress can make it difficult to maintain adequate nutrition. ${ }^{9}$ Fatigue, weakness, and other physical impairments interfere with tasks of daily living. Decreases in physical activity contribute to further immune impairment, increased pain perception, and longer hospitalizations. ${ }^{5}$ Cognitive impairments worsen depression and decrease compliance with treatment. ${ }^{6,8}$ In addition, depression is associated with systemic increases in inflammatory mediators, which can stimulate tumor growth and metastasis. ${ }^{7}$

While these off-target effects are not ideal, in fact they are often detrimental, conventional chemotherapy treatments still represent a substantial improvement in survival compared to no treatment. The challenges presented by treating fatal diseases with equally lethal drugs can only be overcome by improving the specificity of the drugs for the target tissues.

\subsubsection{Targeted Cancer Therapies}


Targeted cancer therapies, which fall under the realm of personalized medicine, have the potential to dramatically improve the response to chemotherapy as well as patient quality of life during treatment. However, the development of these targeted therapies is often quite challenging. Ideally these therapies would "target" tumors by homing on a feature unique to the cancer cells, which would eliminate off-target and systemic effects. Since cancers originate from healthy tissues it can be difficult to identify drug targets that are not present elsewhere in healthy tissues, even if malignant cells use the target molecule or process for a different purpose or in a different amount than healthy tissues. Even most current successful targeted therapies, which have increased specificity over systemic chemotherapy, have off-target effects. Future targeted therapies will rely on the identification of tumor-specific markers and improvements in localized drug delivery.

\subsection{3a Pharmaceutical versus Physical Targeting}

Drug targeting can be considered in terms of both specificity of drug activity and specificity of in vivo drug localization. From a mechanistic perspective, a drug is "targeted" when it acts specifically on a process of interest (Figure 1A). However, even a drug whose action is limited may have widespread biological distribution. Increasing the accumulation of a drug in a specific physical location is, therefore, also a critical component of drug targeting - particularly for drugs that require high local concentrations for effect or those with a high likelihood of off-target toxicity (Figure 1B). 


\subsection{3b Strategies for Targeting Tumors}

Targeted drugs, for conditions other than cancer, are already widely available but not commonly associated with the personalized medicine movement. Antibiotics, for example, are formulated to interact with bacteria-specific enzymes, which minimizes their effects on human cells. The strategies used for targeting bacteria, fungi, and viruses break down when it comes to fighting cancer because we are now faced with identifying "bad self" versus "good self" instead of self versus other.

There are many challenges associated with identifying unique markers of cancer cells when they are located within a body full of normal tissue. Additionally, identifying these markers in vitro does not always translate well to in vivo, where there are often unintended consequences. For example, isotype specific COX blockers were designed to specifically treat pain while minimizing the dose-limiting GI side effects of non-specific COX blockers, but the unintended cardiovascular effects resulted in the drugs removal from the market. ${ }^{12}$

Despite the challenges, some targeted therapies and treatment strategies have been developed and integrated into clinical practice. Current approaches include the use of hormone and hormone-receptor antagonists for estrogen or testosterone sensitive reproductive cancers, ${ }^{13-17}$ inhibitors targeting mutated growth factor receptors in nonsmall cell lung cancer (NSCLC), ${ }^{18-20}$ and inhibitors of a cancer-specific tyrosine kinase fusion protein in chronic myelogenous leukemia $(\mathrm{CML}) .{ }^{21-23}$

Leuprolide and goserelin are gonadotropin hormone releasing hormone $(\mathrm{GnRH})$ agonists. GnRH stimulates the release of luteinizing hormone (LH) from the pituitary gland, which in turn simulates increases in testosterone, DHT, or 
estrogen. ${ }^{13-15}$ Chemical castration, or the complete suppression of estrogen and progesterone production by the ovaries or testosterone production by the testes, can be achieved with both $\mathrm{GnRH}$ agonists and antagonists. ${ }^{13} \mathrm{GnRH}$ agonists have been used to treat metastatic endometrial cancer, while antagonists have been used to fight ovarian cancer. ${ }^{13}$ Both agonists and antagonists are used to treat prostate cancer. ${ }^{14,15}$

Tamoxifen is an estrogen receptor (ER) antagonist used to treat ER positive breast cancer in both men and women. ${ }^{16,17}$ Estrogen acts a growth factor in breast tissue, and blocking its effects can reduce tumor growth and reoccurrence. In patients with ER positive tumors, the addition of tamoxifen as an adjuvant therapy reduced the risk of disease reoccurrence by $47 \%$ and the overall risk of death by $26 \% .{ }^{17}$

Imatinib (Gleevec) inhibits the constitutively active BRC-Abl fusion protein. BCRAbl, encoded by the Philadelphia chromosome $t(9 ; 22)$ reciprocal translocation, is found in over 90 percent of patients with chronic myeloid leukemia (CML) and its activity results in dysregulated cell growth. ${ }^{21-23}$ In CML patients who express BCRAbl, imatinib treatment resulted in $95 \%$ survival at 18 months, with $89 \%$ of patients showing no disease progression. ${ }^{21} \mathrm{~A}$ follow up study reported an $89 \%$ 5-year survival rate..$^{22}$

Gefitinib and Erlotinib are small molecule inhibitors that target the mutated epidermal growth factor (EGFR) tyrosine kinase in non-small cell lung cancer (NSCLC). ${ }^{18-20}$ In populations with a high predisposition for EGFR mutations, 
erlotinib $^{20}$ and gefitinib ${ }^{18,19}$ treatment are more effective than standard combination chemotherapy approaches. When patients are stratified by EGFR status, patients with the target mutation have significantly improved progression-free survival. Patients without the EGFR mutation, as expected, do not experience these benefits.

\subsubsection{Antisense Oligonucleotides as Cancer Therapeutics}

Antisense oligonucleotides (ASO) are powerful tools for regulating cellular protein expression. They allow the production of specific, target proteins to be temporarily turned off. Basic ASO are short fragments of single stranded DNA or RNA, typically 20-30 bases in length, which are complementary to a portion of target protein's mRNA sequence. ${ }^{24,25}$ Structural modification of the bases structure can enhance both the efficiency and stability. When ASO are present in the cytoplasm, they bind to the target mRNA and create a small area of double-stranded nucleic acid that stimulates enzymatic degradation of the mRNA strands as well as physically blocks the translocation of ribosomal machinery. ${ }^{26-29}$

\subsection{4a Regulation of Protein Expression with Antisense Oligonucleotides}

ASO inhibit protein production by binding to their complementary sequence on the mRNA for the target protein (Figure 2). Once bound, ASO work alter protein production by (1) stimulating enzymatic degradation of the double-stranded complex ${ }^{26-28}$ and (2) physically blocking the translocation of the ribosomal machinery along the mRNA. ${ }^{29}$ 
Double-stranded (ds) RNA complexes in the cytoplasm are rapidly detected and enzymatically degraded by RNase $\mathrm{H}^{26-29}$ While the primary function of $\mathrm{RNase} \mathrm{H}$ is to degrade dsRNA, it also recognizes and reacts with the RNA in DNA:RNA hybrids, like those formed by ASO and mRNA. RNase $\mathrm{H}$ interacts non-specifically in the minor groove of the RNA, where three carboxylates from the enzyme associate with the 2'-O-hydroxyl of RNA backbone during the cleavage process. ${ }^{30}$ RNA is then cleaved from the duplex via hydrolysis, leaving a $5^{\prime}$-phosphate and a $3^{\prime}-\mathrm{OH}$ at the cleavage site. ${ }^{27}$ Because the reaction relies on the enzyme's interaction with the RNA 2'-O-hydroxyl group, RNase H will not react with DNA, which does not have the 2'-OH moiety. Theoretically this specificity would protect the DNA of the DNA:RNA complex from degradation, meaning that once the bound mRNA was cleaved the DNA could interact with another target mRNA. However, the ASO will eventually be metabolized by endo or exonucleases within the target cell. ${ }^{26}$

\subsection{4b Delivery of Antisense Agents in vitro}

As nucleic acids are hydrophilic and highly negatively charged, antisense oligonucleotides are generally not able to penetrate cell membranes on their own. ${ }^{31}$ However, they are structurally and functionally similar to siRNA and can be introduced to cells in the same way (i.e. transfection or electroporation). Transfection packages the DNA/RNA in a lipid wrapper, while electroporation utilizes an electric shock to help the oligonucleotide penetrate the cell membrane. 


\subsection{4c Delivery of Antisense Agents in vivo}

Challenges often arise when translating in vitro techniques to clinical settings and ASO are no exception. In fact, the in vivo delivery of antisense agents has, to-date, been largely unsuccessful. However, the potential advantages of antisense agents are great enough that their in vivo delivery is still an area of active research.

Many of the obstacles to clinical implementation are due to limitations of naked oligonucleotides and could be overcome with improved "packaging" for in vivo delivery. ${ }^{31-}$ ${ }^{34}$ For example, naked oligonucleotides are rapidly degraded by plasma nucleases upon intravenous injection. ${ }^{33,34}$ Those that escape degradation are rapidly cleared by the same immune surveillance mechanisms that detect other foreign DNA (like viruses). ${ }^{31,34,35}$ Nucleic acids are generally easily broken down, and unmodified nucleic acids have been shown to have stability issues in salt solutions and aqueous media. ${ }^{36}$ Improved stability is possible with structural modifications to the nucleic acid backbone, including phosphorothioate modifications, ${ }^{31,32}$ locked nucleic acids, ${ }^{31,36}$ and 2'-O-methyl or 2'fluoro modifications. ${ }^{30,31}$

Even if ASO remain intact once they enter the bloodstream, reaching the target tissue requires avoidance of interactions with plasma proteins, clearance by the kidneys, and extravasation at non-target locations. ${ }^{31}$ Assuming the ASO extravasates into the target tissue, naked ASO are not likely to enter cells unassisted. Some plasma protein 
interactions may enhance uptake by stimulating receptor-mediated endocytosis, but typically delivery systems are required for in vivo purposes. ${ }^{31}$

\subsection{4d Clinically Relevant ASO.}

Although ASO have been investigated as treatments for a number of cancer and noncancer diseases, very few ASO are currently available. Currently only 2 ASO therapies have received full FDA approval. However, according to clinicaltrials.gov, there are 43 open or active trials of antisense therapy in addition to the 80 completed trials.

Mongersen is an oral ASO for the treatment of Crohn's Disease that is currently in phase III clinical trials. The 21 base, single-stranded (ss) ASO has a phosphorothioate-modified backbone to increase in vivo half-life. ${ }^{32,37}$ Mongersen targets SMAD7, which is increased in Crohn's disease resulting in suppression of normal anti-inflammatory and immune-suppressive mediators. $\mathrm{A} \mathrm{pH}$ sensitive capsule is used to ensure the oral formulation is not released until it reaches the terminal ileum and right colon (the primary sites affected by Crohn's disease).

Fomivirsen (Vitravene) was the first ASO to receive FDA approval. ${ }^{38}$ It is an injectable, intravitreous ASO for the treatment of cytomegalovirus (CMV) retinitis in patients with AIDS/HIV. ${ }^{38,39}$ The ASO targets the major immediate-early transcriptional unit of CMV, thus interfering with viral replication. ${ }^{39}$ Fomiversen is no longer on the market, as improvements in antiretroviral therapy have reduced the incidence of opportunistic infections like CMV retinitis in HIV/AIDS, but it served 
as in important proof-of-concept drug for the clinical applications of ASO technology. ${ }^{38}$

Mipomersen (Kynamro) received FDA approval in 2013 for use in the treatment of homozygous familial hypercholesterolemia. ${ }^{40}$ The ASO inhibits the production of apopolipoprotein (apo) B-100 in the liver. In healthy patients, mipomersen results in dose-dependent LDL decreases, with a maximum reported LDL decrease of $44 \%$ of baseline. Structural modifications to the ASO give it a half-life of 30 days in circulation. Mipomersen does not interfere with other cholesterol lowering drugs like simvastatin and ezetimibe.

\subsubsection{Future Drug Targets}

Successfully targeted cancer therapies require the identification of molecules or cellular processes that are unique to malignancies. Since cancer develops from healthy tissues, the identification of these unique targets is challenging. Targeted therapies currently on the market, like imatinib (Gleevec), ${ }^{21-23}$ gefitinib, ${ }^{18,19}$ and erlotinib, ${ }^{20}$ exploit structural, and the resulting functional, changes in proteins crucial to cellular regulatory mechanisms. Another class of targets includes proteins that are highly expressed in cancer cells but are not present in most or all healthy cells. The regulation of two such proteins, survivin ${ }^{41-49}$ and mesothelin, ${ }^{50-54}$ has been the focus of many in vitro and in vivo studies in recent years. This work has presented strong evidence that both survivin and mesothelin, independently, contribute to chemotherapy and radiation resistance, more 
metastases, and overall decreased survival rates. ${ }^{43-48,51,52}$ As such, clinical trials have also been pursued for survivin ${ }^{55-65}$ and mesothelin regulating agents, ${ }^{50,51,66}$ although todate no such agents have proven efficacious in humans. Challenges of delivering gene regulatory agents in vivo have been exhaustively documented in relation to treating cancer as well as genetic diseases like muscular dystrophy and cystic fibrosis. As delivery methods improve, the identification and eventual regulation of over-expressed cancerrelated proteins will continue to be an important avenue for the development of targeted therapies.

\subsubsection{Survivin}

Survivin is a cell-cycle dependent inhibitor of apoptosis protein that is heavily overexpressed in nearly all types of cancer but largely undetectable in most healthy tissue. ${ }^{41,42}$ Survivin is overexpressed, to varying degrees, in cancers of the lung, pancreas, colon, prostate, breast, esophagus, liver, stomach, uterus, bladder, and skin, as well as in soft tissue sarcoma, osteosarcoma, melanoma and neuroblastoma. ${ }^{44-48,57,67-}$ 75 Increased tumor survivin has been linked to an increased risk of chemotherapy resistance, more aggressive tumors, and overall worse outcomes. ${ }^{41-49,56,76}$ The prevalence of survivin across cancer types, combined with its association with more aggressive and harder to treat malignancies, makes it an attractive therapeutic target, at least conceptually. ${ }^{49}$ To date, several clinical trials have been carried out with survivin targeted therapies. ${ }^{55-60,77-82}$ Although no survivin-targeted therapies are currently available, therapeutic regulation of survivin is still being actively pursued. 


\section{Survivin Structure and Function}

Survivin is a $16.5 \mathrm{kDa}$ inhibitor of apoptosis (IAP) protein. ${ }^{83-86}$ Structurally, survivin is the smallest member of the IAP family of proteins and contains only one copy of the IAPcharacteristic baculovirus IAP repeat (BIR) domain. In situ, survivin is often found as a homodimer. IAPs, including survivin, can inhibit apoptosis through a number of mechanisms, including direct inhibition of caspase activation ${ }^{87}$ and inhibition of mitochondrial cytochrome $c$ release.$^{88}$ Survivin also plays a role in stabilizing the chromosomes and mitotic machinery during cell division. ${ }^{83-85,89}$

Under physiologic conditions, survivin plays a critical role in normal fetal and placental development, where it acts as a cell-cycle dependent inhibitor of apoptosis. ${ }^{41,42,90}$ Survivin expression is increased beginning in the G1 phase of the cell cycle, reaching a max in the G2-M phase. Survivin interacts with polymerized tubulin, including centrosomes, microtubules, and mitotic spindles, as well as chromosomal kinetochores in metaphase, to stabilize the cell during division, thus inhibiting apoptosis. ${ }^{41,42}$ After cell division, survivin is degraded through the ubiquitin-proteasome pathway. ${ }^{41}$ After birth survivin expression is restricted to the thymus, CD34+ stem cells, and basal colonic epithelial cells. ${ }^{41}$ Most other healthy adult tissues, including peripheral blood leukocytes, lymph nodes, spleen, pancreas, kidney, lung, liver, brain, and heart, have no detectable survivin. ${ }^{43}$ 
In many human cancers, the cells have again acquired the ability to express survivin. ${ }^{41,42}$ Higher levels of survivin are correlated with worse prognosis, increased risk of recurrence and metastasis, and, for some types of cancer, a decreased response to chemotherapy. ${ }^{41-44,56,76}$ Increased survivin is often related to an increased risk of chemotherapy resistance, more aggressive tumors, and overall worse outcomes. A correlation between survivin and tumor aggression/ overall patient outcomes has been reported in colorectal cancer, ${ }^{48}$ bladder cancer,${ }^{67}$ glioblastomas, ${ }^{68}$ melanoma, ${ }^{69}$ non-small cell lung cancer, ${ }^{57}$ breast cancers, ${ }^{70,71}$ diffuse large B-cell lymphoma, ${ }^{44}$ acute myeloid leukemia, ${ }^{44}$ pancreatic ductal cell cancer, ${ }^{72}$ hepatocellular carcinoma, gastric cancer, ${ }^{73}$ malignant gliomas, ${ }^{74}$ soft tissue sarcoma,${ }^{75}$ prostate cancer,,${ }^{45}$ osteosarcoma, ${ }^{46}$ and renal cell carcinoma. ${ }^{47}$

\section{Role of survivin in cancer}

Over-expression of survivin has consistently been associated with apoptosis resistance, reduced sensitivity to chemotherapy, and other markers of tumor aggressiveness both in vitro and in vivo. ${ }^{41-49,56,76,83,85}$ The anti-apoptotic role of survivin allows it to contribute strongly to the development and maintenance of malignant phenotypes. ${ }^{83,85}$ By blocking normal cell death pathways, cellular lifespans are lengthened, which allows mutations to accumulate. The inability of the cells to die contributes to cellular resistance to cytotoxic stimuli, like chemotherapy and radiation, and to metastasis, as the cells are able to survive in inappropriate locations. Conversely, 
survivin inhibition induces increases in spontaneous apoptosis, caspase activation, and sensitivity to cytotoxic stimuli including chemotherapy and radiation. ${ }^{42,44,45,72,76,91}$

Survivin's anti-apoptotic effects are derived, in part, from its role in driving cells through the G2/M cell cycle checkpoint. ${ }^{42}$ Under physiologic conditions, survivin is expressed in cell cycle-dependent manner, with increased expression during the G2/M phase.$^{86}$ In many malignancies, survivin is constitutively expressed but retains the cell cycle-dependent spike associated with the G2/M transition. ${ }^{44,83}$ In this context, survivin plays an important role in mitotic spindle assembly ${ }^{83-85,89}$ and proper chromosome segregation during mitosis. ${ }^{89}$ Specifically, survivin associates with microtubules, polymerized tubulin, centrosomes, and the mitotic spindles as well as Aurora B kinase, a mitotic regulator ${ }^{83,84,89,92}$ Survivin inhibition in cancer cells results in multi-nucleation, failed cytokinesis, and multipolar mitotic spindles. ${ }^{83-85,91}$ Survivin drives cell cycle progression through interactions with CDK4 accelerate $S$ phase, leading to increased cdk2/cyclin E activation and $\mathrm{Rb}$ phosphorylation. ${ }^{84,86}$ Cell-cycle dependent survivin expression is transcriptionally regulated. ${ }^{85}$

While the stabilization of cell replication machinery is an important contributing factor to survivin-induced apoptosis resistance, the presence of survivin in all tumor cells, not just the mitotic fraction, was suggestive of additional anti-apoptotic mechanisms. ${ }^{44,83,85}$ In vitro, survivin expression inhibits apoptosis induced by chemotherapeutic drugs, like cisplatin and etoposide, TNF- $\alpha$, Fas ligand, and caspase 3, 7, and 9.44,45,93 One 
contributing mechanism is that survivin can directly inhibit the activation of caspase- 3 and 7.83-87

A number of factors contribute to cell-cycle-independent survivin expression in cancer cells. The constitutive expression arises, in part, from alterations in the survivin promotor region. ${ }^{83}$ Mutation in the p53 tumor suppressor gene also contribute, as wild-type, but not mutated, p53 functions as a transcriptional repressor of survivin. ${ }^{83,86,94}$ The interaction between survivin and p53 is complex, as survivin can also regulate p53 expression. ${ }^{86}$ Survivin expression can also be induced by dysregulated activation of growth factor receptors, including EGFR, Her2, and insulin-like growth factor-1 (IGF-1), various signaling cascades, and inflammatory cytokines. Aberrantly activated Stat3 and PI3/AKT signaling pathways are known to increase survivin expression, as do IL-11, angopoietin1, and hypoxia inducible factor-1 (HIF-1). Survivin expression can also be induced by environmental factors. Nicotine can activate survivin expression through an AKT pathway in A549 and non-malignant human bronchial epithelial cells. The nicotine-induced increase in survivin expression was noted within 12 hours and persisted for 3 months post-exposure. ${ }^{95}$

\section{Prevalence of Survivin in Cancers}

Histological examination of human tumors has revealed survivin expression in the vast majority of samples, across cancer types and degree of disease progression. For many cancers, tumor survivin expression increases as the disease progresses and higher 
survivin levels are associated with more metastases, chemotherapy and radiation resistance, and overall reduced survival. A summary of survivin prevalence in human cancers is provided in Table 1. Survivin expression in sixty cancer cell lines was previously assessed by Tamm, et al.. ${ }^{96}$

\section{Survivin as a Therapeutic Target}

The relative localization of survivin to tumor tissue, combined with the dramatic success of targeting survivin in vitro, makes survivin regulation seem like an ideal cancer treatment. Decreasing survivin, at least in vitro, results in timely and significant cancer cell death without affecting non-survivin expressing cells. However, replicating this success in a clinically-translatable formulation - or even in in vivo studies - has been challenging.

\section{Regulation of survivin in vitro}

In vitro regulation of survivin expression has primarily relied on ASO and siRNA. ${ }^{83}$ Several effective ASO sequences have been identified, including one from Olie, et al. that has been highly referenced. ${ }^{42}$ Decreases in survivin expression are noted within 24-48

hours of ASO treatment. ${ }^{42,76}$ Using these methods, survivin expression can be reduced to $30-70 \%$ of controls; this variation can be attributed to differences in the basal survivin expression of different cell lines and differences in the potency of the tested ASO. In vitro regulation of survivin with ASO consistently induces apoptosis and increases radiation and/or chemotherapy sensitivity in a variety of survivin-expressing cancer cells without 
affecting non-survivin expressing controls. ${ }^{42,44,72,76}$ The consistent, strong anti-cancer effects seen with in vitro survivin inhibition make it an attractive target for in vivo therapies.

\section{Regulation of survivin in vivo: clinical trials}

\section{Antisense oligonucleotide LY2181308}

LY2181308, the second generation of ISIS 23722 , is a 2'-O-methyoxymethyl modified 18-mer antisense oligonucleotide that decreases survivin expression by binding to the initiation codon of survivin mRNA, resulting in cleavage by RNase $\mathrm{H}$ and degradation of the survivin mRNA. ${ }^{55,60,91,97}$ The resulting decrease in survivin mRNA translates to a decrease in survivin protein expression, which restores sensitivity to normal apoptotic pathways..$^{55,91,97}$

\section{LY2181308: Pre-Clinical Testing}

LY2181308 was identified as the most potent inhibitor of survivin following a screening of 117 2'-MOE phosphorothioate modified oligonucleotides. ${ }^{91}$ In vitro testing ensured that survivin was specifically inhibited, without any effect on other IAPs, in all tested cell lines. It was noted that the decrease in survivin also caused an increase in the activation of caspase-3, a pro-apoptotic mediator. ${ }^{91}$ LY2181308 also inhibited survivin expression and sensitized tumors to gemcitabine, paclitaxel, and docetaxel in human melanoma and glioblastoma xenograft models. ${ }^{91}$ In an in vitro human leukemia model, LY2181308 reduced survivin expression in a dose-dependent manner, with a maximum decrease of 
$40 \%$ within 24 hours and $60 \%$ within 48 hours. ${ }^{98}$ The decreases in survivin expression correlated with decreases in cell proliferation and a G2/M cell cycle block. In an in vivo model of non-Hodgkin's lymphoma, the decrease in survivin expression corresponded with a significant decrease in proliferation, an increase in apoptosis (measured by caspase-3 activation), and smaller tumors. ${ }^{99}$

\section{LY2181308: Clinical Trials}

A first-in-human phase I clinical trial of the antisense oligonucleotide LY2181308 was carried out to assess the biodistribution, tumor tissue penetration, impact on tumor mRNA and survivin protein levels, and dose/toxicity in humans. ${ }^{55}$ The study included 40 participants across a variety of cancer types (Gl, breast, melanoma, lung, sarcoma, ovary, and head and neck carcinoma). 39 out of 40 participants had received chemotherapy prior to the study. During the study, the only drug administered was LY2181308. For this trial, LY2181308 was diluted in 500mL of normal saline and administered IV. It was given daily for three days as a loading dose, and then weekly as a maintenance dose. Tumor biopsies were obtained prior to treatment and 48-96 hours after the third loading dose. While no conclusive results on the in vivo efficacy of LY2181308 were obtained from the study, a $21 \%$ decrease in tumor survivin protein, as determined by immunohistochemistry, and a $20 \%$ decrease in survivin mRNA was reported. The primary adverse effects were flu-like symptoms, including fever, nausea, and musculoskeletal pain, anticoagulation, lymphopenia, thrombocytopenia, hypokalemia, and anemia..$^{55}$ 
In an additional Phase I trial, the dose tolerability, pharmacokinetics, and anticancer activity of LY2181308 were studied in 14 Japanese patients with solid tumors that had not responded to standard therapies. ${ }^{36}$ Japanese patients were studied chosen because the Japanese population, at large, possesses a number of genetic variations that affect the metabolism and safety of anti-cancer drugs, as well as alterations in the TNF gene that affect their response to inflammation. The drug was administered intravenously, in the same manner as in the previous Phase I trial. Adverse effects were in line with those previously reported, including flu-like symptoms during the loading dose phase, prolonged PT-INR and thrombocytopenia, and fatigue. No disease regression was noted in the course of this trial; however, the authors also state that disease regression was not the intention of this trial and that, in the future, this drug would be most likely to succeed when combined with other apoptosis-inducing agents.

Several Phase II trials, investigating the efficacy of LY2181308 combination therapies against non-small cell lung cancer, prostate cancer, and acute myelogenous leukemia, have had similar disappointing results. ${ }^{58-60}$ The three trials described below relied on dosing methodology and schedule from the first-in-human trials of LY2181308. ${ }^{55}$ None of the combination trials reported disease regression following treatment. Adverse effects were noted, but for at least two of the three studies the adverse effects were ultimately linked to the chemotherapy agents that were administered with the LY2181308 rather than the LY2181308 itself. ${ }^{58,60}$ One major limitation of all three combination studies was the failure to enroll patients on the basis of their tumor survivin levels. The small 
number of patients enrolled in each study, combined with the known variations in tumor survivin expression, would make it unlikely that a significant sub-population of drugresponsive patients would emerge. An additional contribution to the lackluster response is that the dosing regimen used in all three studies was only shown to reduce solid-tumor survivin by approximately $20 \%,{ }^{55}$ which may not be a strong enough response to restore apoptosis sensitivity. ${ }^{60}$

\section{YM155: small molecule transcriptional inhibitor of survivin}

YM155 (1-(2-Methoxyethyl)-2-methyl-4,9-dioxo-3-(pyrazin-2-ylmethyl)-4,9-dihydro$1 \mathrm{H}$-naphtho[2,3- $d]$ imidazolium bromide) is an imidazolium-based small molecule inhibitor

of survivin (Figure 3). ${ }^{57,61,65,100,101}$ It acts on the promotor region of the survivin gene, through and unknown mechanism, to specifically decrease survivin protein expression without affecting other IAPs. ${ }^{100}$ Pre-clinical trials of the agent resulted in strong proapoptotic and tumor suppressive effects both in vitro and in mouse xenograft models. ${ }^{100-}$ 102 Phase I and II clinical trials of YM155 as a monotherapy, however, showed little antitumor activity despite evidence that it accumulated in tumors and decreased intra-tumoral survivin expression. ${ }^{57,61-65}$ Future trials of YM155 in combination with other chemotherapy drugs are expected to demonstrate stronger anti-cancer activity than YM155 alone.

\section{YM155: Pre-Clinical Testing}


During pre-clinical testing, YM155 activity was assessed in a panel of 119 tumor cell lines, including 5 known treatment-resistant lines, and a variety of xenograft tumor models. ${ }^{101}$ YM155 treatment, and the subsequent decrease in survivin expression, inhibited cell growth in the majority of the cancer cell lines tested the initial screening panel. Further in vitro studies in hormone-refractory prostate cancer and NSCLC showed that YM155 treatment alone increased spontaneous apoptosis. ${ }^{100,102}$ In vivo, the antitumor effect of YM155 monotherapy was noted in NSCLC, melanoma, bladder, estrogenreceptor-negative breast, and hormone-refractory prostate cancer xenograft models. ${ }^{100,101}$ Decreased intra-tumoral survivin and inhibition of tumor growth were found in all models. One study of NSCLC xenograft tumors found that YM155 inhibited tumor growth more than cisplatin or paclitaxel without affecting mouse weight, and that the antitumor effects were still present three weeks post-treatment. ${ }^{101}$ The synergistic effects of YM155 and radiation were explored with two NSCLC lines, where it was found that treatment with YM155 and radiation together resulted in greater apoptosis in vitro and greater tumor suppression in vivo than either treatment alone. ${ }^{102}$

\section{YM155: Clinical Trials}

A phase I, first-in-human dose study of YM155 assessed the safety and efficacy of the drug in patients with solid tumors and non-Hodgkin's lymphoma. ${ }^{61}$ YM155 was well tolerated and demonstrated a small, but significant, clinical effect. Three of the five nonHodgin's lymphoma patients responded to the therapy, as did two of nine patients with hormone refractory prostate cancer. However, the tumor regression that was seen was 
noted to have occurred at a slower rate than expected with other anti-cancer agents. As a consequence of the slow response rate, the authors urged caution in future trials.

Between a second phase I study ${ }^{62}$ and four phase II trials, the major conclusion was that YM155 was well-tolerated, with the clinical effect appearing questionable at best. In the phase I study, YM155 was administered to 33 patients with various solid tumors and stable disease was achieved in 9 patients. ${ }^{62}$ One phase II study, conducted in patients with advanced, refractory, NSCLC reported "moderate efficacy" of the agent, although they go on to note that they were comparing it to current second-line therapies that also have abysmal response rates. ${ }^{57}$ Another trial, conducted in patients with refractory diffuse large B-cell lymphoma, was terminated early due to futility. ${ }^{64}$ In a third trial, only 2 out of 32 patients with castration-resistant prostate cancer had a clinically measurable response, and 26 of the initial 32 participants withdrew due to treatment failure. ${ }^{63} \mathrm{~A}$ trial of YM155 in patients with advanced melanoma also failed. ${ }^{65}$

The failure of YM155 to suppress tumors in clinical trials was attributed primarily to the tests assessing its efficacy as a monotherapy, with the suggestion that it would likely be more effective as part of a combination treatment. ${ }^{61-65}$ Its mechanism involves sensitizing cells to cytotoxic stimuli or apoptosis from mitotic failures. Thus, without any additional stimuli, the treatment would only effect cells that were actively dividing. ${ }^{63}$ Additionally, there are anti-cancer drugs currently used that are not effective as monotherapies. 


\section{Survivin Peptide Vaccine}

Survivin overexpression in human tumors elicits a T-cell immune response. Circulating anti-survivin antibodies and survivin-reactive T-cells have been documented in patients with leukemia, lung, colon, breast, and pancreatic cancers, but not in healthy control subjects. ${ }^{80,85,91,103}$ Processed survivin peptide fragments are displayed on human leukocyte antigen (HLA) class I molecules on the cell surface, where they interact with cytolytic T-cells. The presence of survivin reactive T-cells has generated interest in the development of survivin peptide vaccines. ${ }^{83,85,86,91}$ To those ends, survivin intracellular processing was studied and the immune-reactive peptide fragments were enumerated ${ }^{104}$ and the identified fragments were used to generate survivin peptide vaccines. ${ }^{56,77-82}$ While the survivin peptide fragment that generates the strongest T-cell response will vary by HLA type and other patient-specific factors, cancer type is not a limiting factor. Survivin-reactive cytotoxic T-cells isolated from leukemia patients reacted with other survivin-expressing tumors cells, including breast cancer, renal-cell carcinoma, colon cancer, melanoma, multiple myeloma, and primary malignant cells from other leukemia patients. ${ }^{80,105}$

Survivin peptide vaccines have been tested in a number of late-stage cancers, including metastatic melanoma, lung cancer, and pancreatic cancer, to demonstrate their safety and ability to induce a survivin-specific T-cell response. ${ }^{56,77,79,81,82}$ In a case study of a HLA-A2 restricted survivin peptide, a single patient with advanced metastatic pancreatic cancer experienced complete tumor regression after 14 months of weekly vaccination. He remained disease free for an additional 8 months, at which point new 
metastases were discovered and the patient rapidly declined. ${ }^{56}$ In patients with stage IV metastatic melanoma, HLA-type specific vaccines were developed for HLA-A1, -A2, and -B3 positive tumors. ${ }^{77}$ It was found that patients who developed the survivin-specific Tcell response had longer survival times than patients that did develop survivin-specific reactivity (median survival 19.6 vs 8.6 months). In addition, patients who developed a post-vaccination inflammatory response, including fevers, had stronger T-cell reactivity and improved survival than patient who did not develop an inflammatory response.

Although survivin-targeted vaccines appear safe and are able to induce a T-cell response, the clinical utility of these therapies is still unknown. Trials in heavily pre-treated patients with metastatic disease could mask the potential of vaccination-based therapies since many cancer treatments suppress the immune mechanisms required for effective vaccination. Also, most of the trials so far have utilized single-epitope vaccines, which were chosen based on patient HLA type. ${ }^{78}$ However, even patients with the same HLA type have dramatic variations in their responses to single survivin epitopes. It has been suggested that vaccines should contain multiple survivin epitopes to allow for personalized responses.

\subsubsection{Mesothelin}

Mesothelin is a cell-surface protein of unknown physiological significance ${ }^{106}$ that is overexpressed in approximately $30 \%$ of all cancers, ${ }^{50}$ including nearly all mesotheliomas ${ }^{50}$ and many lung,,$^{51,52}$ pancreatic, ${ }^{51,53}$ and ovarian ${ }^{51,107}$ carcinomas. Significant levels of mesothelin are not present in healthy tissues, ${ }^{53,54}$ although low levels 
are expressed in normal mesothelial cells \& epithelial cells of the fallopian tubes, kidneys, trachea, and tonsils. ${ }^{107}$ While the specific function(s) of mesothelin are currently unknown, its presence in malignancies has been linked to more aggressive tumor behaviors and chemotherapy resistance. ${ }^{51,52}$

\section{Structure and Function.}

The mesothelin gene encodes a $71 \mathrm{kDa}$ precursor protein that is cleaved in the cytoplasm to give two functional products: the $40 \mathrm{kDa}$ mature mesothelin cell-surface protein (MSLN) and a $31 \mathrm{kDa}$ soluble fragment that is released into the blood (SMRP). ${ }^{51}$ The mechanisms behind MSLN's contribution to cancer development and progression are not yet known; however, MSLN appears to affect a number of critical cell processes including cell adherence, ${ }^{51}$ proliferation, ${ }^{51-53}$ invasion and migration, ${ }^{52,108,109}$ and apoptosis resistance. ${ }^{51}$ The SMRP is clinically detectable in the plasma of patients with MSLN over-expressing malignancies, and serial measurements of SMRP is under investigation as a clinical tool for tracking response to treatments and monitoring for disease reoccurrences in lung adenocarcinoma, mesothelioma, ${ }^{110}$ pancreatic cancer, ${ }^{51,53}$ and ovarian cancer. ${ }^{66}$

Mesothelin over-expressing cancer cell lines proliferate faster than their mesothelindeficient counterparts, both in vitro and in vivo in mouse xenograft models. In lung cancer cell lines, mesothelin positive cells proliferated 2-3 times faster than mesothelin negative cells. $^{51-53}$ IL-6, an inflammatory chemokine, is thought to be a mediator between 
mesothelin and some of the associated aggressive behaviors. IL-6 promotes cell growth, chemo-resistance, anchorage-independent cell growth, and invasiveness ${ }^{111,112}$ and is over-expressed in MSLN-expressing pancreatic cancer cells. ${ }^{51,112}$ Cyclin E, a cell cycle regulator that promotes the G1/S phase transition, is frequently dysregulated in cancer cells, ${ }^{113}$ and mesothelin may contribute to increased proliferation rates by inducing cyclin E overexpression through an IL-6/Stat3 pathway. ${ }^{114}$

Tumor mesothelin expression has also been linked to increased invasion and migration in vitro, which translates to increased metastatic ability in vivo. In vitro, induced overexpression of mesothelin increased invasion and migration in lung and pancreatic cancer cell lines, ${ }^{51-53}$ and mesothelin-induced increases in matrix metalloproteinase (MMP) expression have been linked to increased invasiveness in ovarian cancer cells. ${ }^{109}$

Increases in invasion, migration, and metastases may be the result of mesothelinrelated changes in cellular adhesion. ${ }^{115,116}$ Interactions of mesothelin with the ovarian cancer antigen CA-125 have been described, and these interactions are thought to potentiate the spread of ovarian cancer to the peritoneal lining. ${ }^{51,117}$ In a mouse model of kidney tumors, mesothelin expression increased cell adherence to collagen coated plates, while mesothelin inhibition decreased adherence to collagen. ${ }^{115,118}$

In both physiologic conditions and tumor samples, mesothelin expression has been primarily localized to the apical membrane of the cells. ${ }^{108,117,119-123}$ In invading tumor specimens, this can correlate to the leading edge of the tumor. Although the physiologic 
function of mesothelin is not known, the apical localization, taken together with what is known about mesothelin over-expression, suggests that mesothelin could have a role in the barrier functionality of mesothelial linings.

Prevalence in Cancer. MSLN is overexpressed in approximately $30 \%$ of human cancers; however, the prevalence varies depending on the cancer type. ${ }^{50}$ An excellent summary of MSLN expression in human cancers is presented in Mesothelin targeted cancer immunotherapy. ${ }^{124}$

Lung Adenocarcinoma. MSLN is commonly expressed in lung adenocarcinomas, including stage 1 patients. ${ }^{52}$ In an analysis of 1252 patient samples, $69 \%$ of patients had detectable levels of MSLN in tumor, with no MSLN staining in normal lung tissue or tumor stroma. ${ }^{52}$ The same study found that MSLN was an independent predictor of relapse and overall survival, and higher MSLN expression was associated with both male sex and a positive smoking history.

When patient tumors were analyzed, it was found that lung cancer samples seemed to have more intracellular MSLN than ovarian cancers, mesotheliomas, and normal mesothelial cells. The lung cancers were also noted to have more mesothelin precursor protein than other cancer types. ${ }^{54}$ Strong cytoplasmic and membranous staining was also noted by Kachala, et al. ${ }^{52}$ 
Mesothelioma. Mesothelin is highly expressed by nearly all mesotheliomas. ${ }^{50,51}$ However, the SMRP is only detectable in $40-70 \%$ of patients, ${ }^{50,66}$ making it of questionable prognostic value. It has been noted that SMRP levels are higher in advanced-stage patients than early-stage patients. ${ }^{125,126}$

Ovarian Cancer. Mesothelin is overexpressed in approximately $70 \%$ of ovarian cancers and contributes to decreased progression-free and overall survival. ${ }^{51,66,107,127}$ Higher levels of tumor mesothelin expression have also been correlated with chemotherapy resistance. ${ }^{107} \mathrm{CA}-125$ is often co-expressed with mesothelin, and coexpression results in worse outcomes than either antigen alone. ${ }^{110}$

Other Cancers. Mesothelin overexpression has also been documented in uterine serous carcinoma, ${ }^{51}$ acute myeloid leukemia, ${ }^{51}$ cholangiocarcinoma, ${ }^{51}$ squamous carcinomas of the esophagus and cervix, ${ }^{128}$ pancreatic adenocarcinoma, ${ }^{50,51}$ and triple negative breast cancers. ${ }^{50}$

\section{Mesothelin as Therapeutic Target.}

\section{SMRP as Cancer Biomarker.}

SMRP is released into systemic circulation when the mesothelin precursor protein is cleaved to generate mature mesothelin. Since it is easily detectable in patient plasma, using commercially available ELISA kits, its potential as a clinical cancer biomarker has been investigated. ${ }^{50,129}$ Serial SMRP levels could, theoretically, be used as a non- 
invasive tracking tool to monitor for response to treatment and disease relapse. ${ }^{50,51,53,66,110}$ SMRP has been detected in patients with mesothelioma, ${ }^{50,110,129}$ pancreatic cancer, ${ }^{51,53}$ and ovarian cancer, ${ }^{66}$ but it is currently of debatable clinical utility. ${ }^{50,129,130}$

\section{Therapeutic Targeting: Mesothelin Antibodies and Vaccines}

Mesothelin-targeted immunotherapies have been developed using both immunotoxins and mesothelin vaccines. ${ }^{124,127}$ Ongoing clinical trials, targeting mesothelin through immunotherapies, have not resulted in adverse effects and the therapies have not damaged normal tissues. ${ }^{50}$

\section{SS1P: Mesothelin Immunotoxin}

The anti-mesothelin immunotoxin SS1P utilizes a recombinant anti-mesothelin antibody linked to a truncated Pseudomonas exotoxin to target cell-surface mesothelin. ${ }^{124}$ SS1P binds to mesothelin on the cell surface, is internalized via clathrin-coated pits, and the exotoxin is freed during endosomal processing. The toxin migrates to the endoplasmic reticulum and inhibits elongation factor-2, leading to inhibition of protein synthesis and cell death. Initial studies demonstrated the in vitro cytotoxicity of SS1P against tumor cells from patients with mesothelioma and ovarian cancer. ${ }^{131-133}$ In mice, SS1P induced the complete regression of human epidermoid carcinoma tumor xenografts. ${ }^{131,134}$ Additional 
mouse studies demonstrated that SS1P can act synergistically with radiation and chemotherapy, including paclitaxel, cisplatin, and cyclophosphamide. ${ }^{124,131,135,136}$

In a phase I dose escalation study, minor tumor responses were noted in 4 patients while stable disease was achieved in 19 (56\%) patients. ${ }^{124,131}$ The major adverse effect was minor, self-limiting pleuritis from SS1P binding to the mesothelin present on normal mesothelial cells. The limited success of SS1P in clinical trials was attributed to patients developing antibodies to the immunotoxin. A follow-up study treated patients with chemotherapy refractory mesothelioma with pentostatin and cyclophosphamide, in addition to SS1P, to delay the development of anti-SS1P antibodies. ${ }^{137}$ Of the ten patients in the study, 3 experienced major tumor regressions and 2 responded to chemotherapy after treatment with the immunotoxin. This improvement is considered significant in light of poor prognosis associated with the patients' disease state. Stronger anti-tumor activity is possible if SS1P is given intraperitoneally, particularly when treating ovarian cancer or peritoneal mesothelioma, or given in combination with chemotherapy or radiation. ${ }^{131}$

\section{Mesothelin Vaccines}

Anti-mesothelin immune responses have been detected in cancer patients, leading to the hypothesis that an immune response to mesothelin could be induced via vaccination. ${ }^{124,138}$ Antibodies against mesothelin are present in many patients with 
mesothelioma and ovarian cancer, while anti-mesothelin T-cell responses have been noted in patients with pancreatic cancer. ${ }^{66,124}$

CRS-207, a mesothelin cancer vaccine using live attenuated Listeria monocytogenes as the vector, is in phase I testing in patients with carcinoma and liver metastases. ${ }^{124}$ Vaccination of both mice and monkeys with CRS-207 elicits a human-specific T-cell response, and anti-tumor activity has been demonstrated in mice.

MORAb-009 is a monoclonal antibody targeting mesothelin that induced a moderate response against mesothelin-expressing tumors in a phase I clinical trial. ${ }^{139}$ Of the 24 patients enrolled, 11 achieved stable disease. In vitro, MORAb-009 was cytotoxic as a single agent to mesothelin-expressing cancer cells and synergistic cytotoxicity was seen when it was used in combination with gemcitabine and paclitaxel. ${ }^{139,140}$ MORAb-009 also reduced the adhesion between mesothelin-expressing cells and CA-125. ${ }^{140}$ 


\subsection{Evaluating Nanotoxicity}

\subsubsection{Introduction to Nanoparticles}

Nanoparticles (NPs) are 1 to 100 nanometers (nm) in at least one dimension. ${ }^{141-145}$ The small size of the NPs confers unique properties when compared to the same material in bulk, including changes in color, solubility, electrical conductivity, and magnetic properties. ${ }^{144-146}$ In addition, NPs have a large surface area to volume ratio, which increases their surface reactivity. ${ }^{141,144,146}$ The unique physical and chemical properties of NPs have resulted in their rapid incorporation into a variety of consumer goods. Additional industrial, technological, and biomedical applications are the focus of ongoing research.

NPs used in commercial products include silver NPs in clothing, ${ }^{142}$ titanium dioxide $\left(\mathrm{TiO}_{2}\right)$ in cosmetics, sunscreens, and food packaging, ${ }^{144}$ and carbon nanotubes (CNTs) in sports equipment and micro-electronics. ${ }^{147}$ Superparamagnetic iron oxide nanoparticles (SPIONs) received FDA approval for use as MRI contrast agents ${ }^{148-151}$ and for the treatment of anemia in patients with chronic kidney disease. ${ }^{150,152}$ The development of NP-based biosensors, imaging modalities, and targeted and multifunctional therapeutics is an area of active research. ${ }^{145,153,154}$

Despite the widespread incorporation of NPs into consumer goods, their toxicity is not well understood. ${ }^{141-144,155,156}$ It has been demonstrated that bare NPs are often more toxic than equivalent doses of bulk material, perhaps as a result of the increased surface 
area to volume ratio. ${ }^{141,144,146}$ For example, nano-sized $\mathrm{TiO}_{2}$ induces more pulmonary inflammation than larger particles at the same dose. ${ }^{144}$ However, coated or functionalized NPs, when appropriately designed for biological purposes, are considered biocompatible. ${ }^{143,145,154,157-161}$

These contradictory safety reports have impeded the incorporation of NPs into additional goods and technologies. The ability to draw conclusions from much of the existing research into NP biocompatibility is limited because of a lack of standardization in NP characterization, doses, exposure routes, and toxicity metrics. ${ }^{141-144}$ NPs can also interfere with many of the standard toxicity assays. ${ }^{142,144,155,156,162,163}$ Understanding the relationship of particle NP composition and physical properties to toxicity, biodistribution, clearance, metabolism, and mechanisms of toxicity will pave the way for clear occupational safety standards and the introduction of biocompatible engineered NPs for clinical use. However, overcoming the obstacles associated with achieving this understanding will require a shift from traditional approaches to toxicity testing.

\subsubsection{Nanoparticle Toxicity}

Determination of NP toxicity introduces new challenges compared to evaluating the toxicity of small molecules or larger particles. Although NPs are not a singular class for toxicity purposes ${ }^{141}$ - in fact there are dramatic variations in the toxicity of NPs within the same class when physical properties are altered - the challenges of assessing nanotoxicity are consistent across all NP types and applications. ${ }^{141,143}$ The lack of 
standardization in NP characterization and toxicity assessment has impeded the ability to compare and parse the often contradictory conclusions reported in the literature. ${ }^{141-}$ 144,164,165 Additionally, NP toxicity studies are rarely reflective of real world exposure conditions. ${ }^{141,155,165}$ Relevant particle size distribution and impurities, doses, exposure routes, and incubation times are often difficult to discern ${ }^{141-143,166}$ and NPs can interfere with many standard toxicity assays. ${ }^{142,144,155,156,162,163}$ Although the need for validated models of NP characterization and toxicity assessment has become apparent, ${ }^{141,144,162,167}$ such models do not yet exist.

\subsubsection{Role of NP Physical Properties}

NP toxicity, and the mechanism of that toxicity, is influenced by particle physical properties, including particle size, shape, aspect ratio, hardness, composition, surface area, surface chemistry, coatings and modification, and stability in solution. ${ }^{141,146,155,162,164}$ When NPs are engineered for biomedical applications, the final system properties are chosen to direct in vivo distribution and accumulation while minimizing clearance and toxicity. ${ }^{153,168}$ On the other hand, NPs involved in occupational or environmental are engineered to maximize industrial performance and ease of synthesis, without regard for biocompatibility. As a result of the relationship between NP physical properties and toxicity, NPs used in toxicity assessments need to be fully characterized. ${ }^{141,146}$ The relationship of physical parameters to biocompatibility of biomedical NP platforms is discussed in Section 1.1.3 (Role of NP Physical Properties), while the relationship of CNT 
physical properties to toxicity is discussed in 1.3.3 (Role of CNT Physical Properties in Toxicity).

\subsubsection{In Vitro Toxicity Assessment}

Much of the work evaluating the biocompatibility of NPs has been carried out in vitro. In vitro assessments are used as a first-line screening approach because they can be carried out faster, and with less expense, than in vivo studies. ${ }^{155}$ In vitro toxicity is often quantified by measuring changes in cell proliferation, viability, membrane integrity, and cell functions that would be impacted by the NPs mechanism of action, like ROS generation and DNA damage. ${ }^{141,143,155}$ In vitro studies also allow for quantification of NP uptake, determination of the mechanism of uptake, and intracellular localization. ${ }^{142,162,169}$ However, NPs have been noted to interfere with a number of traditional toxicity assays, including the several widely utilized measures of cell viability and membrane integrity (Table 2). ${ }^{142,156,162}$ As a result, it is recommended that multiple assays are used to validate in vitro results. ${ }^{162}$

Unfortunately in vitro test conditions are not always reflective of in vivo results or real world exposures. ${ }^{142,165}$ For example, unrealistically large NP doses are often used in single-dose treatments to simulate long-term occupational exposures and accumulation. ${ }^{146,155,166}$ Thus, many of the in vitro screenings are cursory looks at changes in cell viability, proliferation, or apoptosis after acute, high-dose exposures ${ }^{141,143,155,170,171}$ When NPs are intended for use as therapeutics these measures might be reflective of 
actual exposures (controlled dose of a well-described material), but for occupational exposures chronic, low-dose exposures are more likely. ${ }^{171}$ To overcome some of these limitations, chronic in vitro exposure models have been developed that account for longer term, lower dose occupational exposures like those associated with CNTs. ${ }^{172,173}$

\subsubsection{In Vivo Toxicity Assessment}

In vivo exposure models are used to determine NP biodistribution, clearance mechanisms, and systemic and target organ toxicity. ${ }^{155}$ In vivo models overcome the limitations that arise in vitro from the use of single-cell types, limited exposure times, and artificially high NP accumulation when NPs settle on cultured cells. ${ }^{142}$ NP distribution can be assessed from organ analysis or radiolabeled or fluorescently-tagged NPs can be used for real-time in vivo tracking. ${ }^{155}$ However, radio- or fluorescent labels can change the surface properties of the NPs and affect their distribution and clearance. Systemic toxicity can be monitored through changes in blood composition, changes in serum proteins that would signify target organ damage, or changes in inflammatory mediators.

\subsubsection{Dosing NPs for Toxicity Evaluations}

Determining appropriate NP dosing is problematic in both in vitro and in vivo studies. Complex, multi-functional NP systems challenge traditional dosing paradigms, as all components, and their relative amounts, orientations, and effects on the NP systems physical properties, must be taken into account. Thus, fully elucidating the composition 
of complex NP systems is critical to obtaining meaningful, reproducible, dose-dependent toxicity data. CNT inhalation exposure is a major concern in industrial settings. ${ }^{146,174-176}$ CNTs used in industrial applications are typically mixtures containing a variety of particle shapes, sizes, agglomeration states, and metal impurities. ${ }^{141,142,155,163,177}$ This variety is difficult to mimic during laboratory testing, but a thorough evaluation of the particles that are being tested will allow for more informed extrapolation to real world conditions. NP for use in biological systems as contrast agents, drug delivery vehicles, or biosensors, are often coated and functionalized to improve biocompatibility, circulation time, and therapeutic efficacy. ${ }^{143,145,153,154,177,178}$ The addition of these coatings and functional ligands creates a complex, multilayer system composed of combinations of surfactants, amphiphilic polymers, linker molecules, fluorescent dyes, and biomolecules surrounding the NP core. In this conditions, the relative amounts of the components become important. Ideally the components will combine in constant ratios, allowing consistency in dosing across batches regardless of the dosing measure, but such consistency is rare even in commercially available products.

\subsubsection{Dosing Considerations: NP Quantification \& Dosing Metrics}

Many NPs for biological applications, including SPIONs, contain several organic layers built around a metal oxide core. While quantification of the various components within a given sample is possible, there is no way to ensure that each particle is uniformly coated or functionalized. Although the variation likely averages out over a large enough dose or sample size, the inherent heterogeneity and complex makeup necessitates careful consideration of the appropriateness of the chosen dosing measure. Commonly 
used dose measures include concentration of the metal in the core, ${ }^{179-181}$ particle mass, ${ }^{182,183}$ or molarity. ${ }^{157,159}$ When therapeutic ligands, such as ASO, ${ }^{184}$ aptamers, ${ }^{185}$ antibodies, ${ }^{160}$ or targeting molecules like folate ${ }^{186-190}$ are present, their concentration should also be considered. In many situations, the most appropriate dosing measure will be a combination of two or more measures, ensuring that all relevant components are accounted for.

Although mass and molarity are the most obvious dosing methods, particularly from the viewpoint of conventional toxicity assessments, their use with complex, multifunctional NP systems has a number of disadvantages. The major disadvantage to massbased dosing is that particle coating and functionalization has a significant impact on mass. As a result, equivalent masses of bare, coated, or differently functionalized NPs would contain drastically different numbers of particles. In one study dosed by mass, the bare NPs were $75 \%$ core and $25 \%$ surfactant by mass, while the coated particles were $15 \%$ core and $85 \%$ surfactant/coating. ${ }^{182}$ Consequently, cells treated with the bare NPs were exposed to 5 times more particles than cells treated with coated particles. Thus the use of mass as a dosing metric makes it difficult to determine if any observed differences are the result of the change in composition or the vastly different number of particles between the two samples.

Dosing by molarity assumes that the composition of the NP system is consistent, which it rarely is, and then the mass of a mole must be calculated or defined; however, 
when molarity is used in NP toxicity assessments, it is almost never explained how a mole of the NP-platform was defined.

When NP doses are calculated by the concentration of the metal in the particles core, the number of NPs is kept consistent regardless of the coatings. This overcomes one of the major limitations of dosing by mass. However, inconsistent amounts of coatings or ligands are not accounted for. In addition, determining the metal concentration of a sample is destructive and may require an impractically large sample size when NPs are functionalized in small batches.

If NPs are functionalized with an easily quantifiable therapeutic ligand, like an ASO, the ligand concentration can be used to keep the expected effect constant. Dosing based on ligand concentration allows for comparisons to previous studies of the ligand alone, for example the efficacy and timing of a naked ASO can be compared to a NP-ASO conjugate. In cases where the ligand does not couple consistently to the NP platform, dosing in this manner can help normalize data to the actual amount of ligand.

In an ideal scenario, several of these measures would be combined to allow for consistent, reproducible, and easily explained NP dosing. Consistent relationships between NP components would allow one measure to be used, while the relative amounts of the remaining components could be calculated for comparison across studies. For NPs with inconsistent ratios of components, measuring and reporting the relative amounts of all components will allow for more meaningful comparisons between studies and will 
contribute to improved understanding of the roles of various components in toxicity and therapeutic effects. Fully described NP systems will help overcome initial limitations in interpreting nanotoxicity literature, where incomplete characterization reports ${ }^{144}$ and inconsistent dosing made it nearly impossible to compare experimental results. ${ }^{141,155,173}$

\subsubsection{Dosing Considerations: Modeling Real World Exposures}

For occupational exposures, dosing and exposure routes should be chosen to closely approximate real world human exposures. ${ }^{141,165}$ In determining test dosages, occupational exposure limits, documented human exposures, and the time course of the exposure should be considered. In addition, conversions from in vitro to in vivo exposures should account for exposed surface area rather than relying on solution concentrations. ${ }^{166}$ When modeling chronic NP exposure in vitro, long-term exposure to low-doses ${ }^{172,173,175}$ may be more representative than a single large dose. ${ }^{166}$

\subsubsection{Strategies for Evaluating NP Toxicity}

It is already impossible to avoid exposure to NPs and their incorporation into everyday products is rapidly increasing. Developing methods for thorough, reliable evaluation of NP acute and long-term toxicity is critical to harnessing their full potential for industrial and biomedical applications. However, evaluating the toxicity of NPs requires critical evaluation of suitability of standard approaches. 
Quality control during NP synthesis and functionalization is of the utmost importance, as intra- and inter-batch variation can have a dramatic impact on observed toxicity. In addition, NPs are often synthesized under non-sterile conditions, resulting in frequent contamination with bacteria or endotoxin that can cause false positives or increases in observed toxicity and inflammatory responses. ${ }^{144,191}$ Once the NPs are properly formulated, their stability in storage solutions must be monitored. ${ }^{145,146}$ During biological testing, changes in NP aggregation or solubility can arise from changes in $\mathrm{pH}$ or interactions with biomolecules in culture media. ${ }^{191}$ The adsorption of proteins from culture media can also affect particle uptake or toxicity, meaning that culture conditions need to closely approximate actual exposure conditions. ${ }^{145,153,169}$ The interplay between the physical properties of the NPs and their storage or treatment conditions necessitates monitoring for quality control through the NPs entire laboratory lifespan. The optimal conditions for each NP formulation may vary, meaning a one size fits all approach to evaluating toxicity will have little relevance.

The generation of meaningful, relevant nanotoxicity data will require approaches that integrate knowledge of the NP chemical and physical characteristics with the biological test conditions and the mechanisms behind toxicological assays. The current paradigm of purchasing a compound and testing it as is, which works for small molecules, needs to shift to one with improved access to and understanding of quality control measures and monitoring. The lack of characterization data is most apparent in NPs engineered for biomedical applications, where there is hand-off from the scientists who prepare the NPs to those who test them. It seems that there are well-characterized NPs where the 
biological applications are an afterthought, or detailed biological experiments where little consideration is given to the NP design, properties, or dosing.

Improvements in the determination and reporting of the physical properties of these complex NPs will require increased collaborations between fields. Continued communication between the scientists who make the NPs and those who test them can also help identify or resolve problems with assay interference before miscalculated results are released. The ongoing communication, full NP characterization, and ensuring formulation consistency will initially increase the time required for NP toxicity assessments; however, the improvements will eventually save time and money by reducing the conflicts and confusion surrounding published toxicity data. 


\subsection{SPION-Based Therapeutics}

\subsubsection{NP Therapeutics}

There has been great interest in the development of NP-based agents for drug delivery and diagnostics, particularly in fields like oncology where there is an undeniable need for improved efficacy with reduced side effects. ${ }^{152,153}$ Many current therapeutic agents have low solubility, low stability, and are rapidly cleared from circulation. Rapid clearance necessitates frequent administration to maintain therapeutic concentrations. ${ }^{153}$ Conjugation of these drugs to polymers can improve circulation half-life, reducing the frequency of administration. Generally, untargeted NPs will accumulate passively in tumors or areas of inflammation due to leaky vasculature and poor lymph clearance. The addition of biomolecules or targeting ligands can increase therapeutic efficacy or increase accumulation in target tissues. ${ }^{150,186,192-196}$ To those ends, NPs can be functionalized with DNA or RNA oligonucleotides, peptides, antibodies, fluorescent dyes, polymers, or drugs. ${ }^{145,150,186,192-196}$ However, NP functionalization changes particle size, surface charge, hydrophobicity, and biodistribution, so toxicity assessments will require the final, fully characterized NPs.

\subsubsection{Therapeutic NPs: Design Considerations}

NPs for therapeutic applications must be carefully designed to evade normal clearance mechanisms. Typically hepatic filtration removes large particles, while renal filtration removes small particles. ${ }^{152,153}$ In addition, NP interactions with various plasma 
proteins can drive rapid clearance. ${ }^{142,144,145,162,191}$ NP coatings, including poly(ethylene glycol), and surface functionalization can be strategically chosen to minimize undesirable plasma protein interactions and maximize distribution to target tissues and cellular internalization. ${ }^{145,150,151,153}$ Thus careful engineering of particle size, shape, and surface charge improves biocompatibility and circulation half-life while contributing to increased therapeutic efficacy.

\subsection{2a NP Size}

When NPs are engineered for biomedical applications, the final system dimensions are chosen to direct in vivo distribution and accumulation while minimizing unwanted clearance. ${ }^{153,197}$ Ideally NPs for intravenous (IV) administration should be 10-100 nm. NPs that are less than $10 \mathrm{~nm}$ are rapidly filtered by the kidney, while those larger than $100 \mathrm{~nm}$ are cleared via the liver and spleen. When NPs are intended to accumulate in target tissues, their ability to exit circulation also needs to be considered. Normal blood vessels are tightly sealed, but the rapid growth and inflammation associated with tumors results in porous vasculature that allows NPs up to $400 \mathrm{~nm}$ to extravasate.

\subsection{2b Minimizing Plasma Protein Adsorption}

NPs are also rapidly cleared from the circulation by phagocytic cells such as macrophages. Phagocytic clearance is stimulated by the adsorption of specific plasma proteins to the NP surface. ${ }^{142,144,145,153,162}$ The addition of inert, biocompatible surface 
coatings, like poly(ethylene glycol) (PEG), dextrans, or synthetic block co-polymers increases circulation half-life by reducing plasma protein interactions and, thus, minimizing clearance by macrophages. ${ }^{145,150,151,153,154}$ Without modification, most NP drug carriers are cleared from circulation within minutes. ${ }^{154}$ When neutral, hydrophilic coatings are employed, half-life can be extended to upwards of 2 hours. ${ }^{154}$

\subsection{2c NP Surface Charge}

NP surface charge also affects particle uptake, internalization mechanism, and intracellular distribution, even after controlling for NP size and shape. ${ }^{169}$ In general, positively charged particles have a higher rate of cell uptake compared to negative or neutral surface charges. ${ }^{153,169,198}$ However, positively charged NPs are also more cytotoxic than NPs with negative or neutral surface charges. ${ }^{157}$ Differences in uptake mechanism have been noted in vitro, where positively charged NPs accumulate quickly on cell surfaces, likely due to electrostatic interactions with the negatively charged cell surface proteins, and are taken up via adsorptive endocytosis in a concentrationdependent manner. ${ }^{169} \mathrm{NPs}$ with a negative surface charge, that were otherwise of similar size, shape, and composition to the positively charged ones, were taken up at a steady and constant rate, independent of their membrane concentration, by common endocytosis. In vivo, NPs with a positive surface charge have higher rates of non-specific internalization, perhaps due to their electrostatic attraction for the negatively charged cell membrane proteins, and have a shorter circulation half-life. ${ }^{153}$ NP surface charge also indirectly affects in vivo uptake and distribution, as positive and negatively charged NPs 
have different plasma protein interactions. Negatively charged NPs are more likely to interact with proteins that have specialized cellular uptake mechanisms. ${ }^{169,191}$

\subsubsection{NP Targeting}

When no targeting mechanisms are employed, intravenously administered NPs that evade clearance will distribute primarily to the liver, spleen, kidneys, and tissues with leaky vasculature, which includes tumors and areas of inflammation. ${ }^{152,154,199}$ This passive distribution is affected by changes in NP size and surface charge, as the major driving force in the distribution pattern is size-dependent extravasation through vascular fenestrations and size-dependent clearance by the liver, spleen, and kidneys. ${ }^{150,192}$ Improved control over NP localization can be achieved with surface functionalization.

Conjugation of the NP with ligands targeting tumor specific cell-surface receptors, proteins, or uptake reduces non-specific uptake and enhances concentration at target tissues. ${ }^{145,153,192}$ For example, folate-conjugated NPs were taken up by tumors at a greater rate than surrounding tissue. ${ }^{160,186-190,200}$ The addition of an aptamer targeting prostate-specific membrane antigen (PSMA) to a doxorubicin-loaded SPION created a system that was cytotoxic only to PSMA-expressing cells. ${ }^{185}$ Antibodies are also highly specific and can significantly increase accumulation within target tissues. ${ }^{145,201,202}$ However, antibodies require proper conformation and orientation to recognize their targets. Conformation and orientation are difficult to maintain or guarantee during the coupling process. In addition, antibodies are immunogenic and may stimulate an 
undesirable increase in particle clearance. ${ }^{154}$ Consequently, whole antibodies are not typically used; rather, when antibodies are employed, small fragments containing the variable region are used instead. ${ }^{154}$

\subsubsection{NP Surface Modification Strategies}

NP therapeutics are generally complex, with multiple layers surrounding the NP core. For SPIONs, the NP core is typically coated in a surfactant, like oleic acid, which is then coated with an amphiphilic polymer to improve aqueous solubility and provide reactive sites for further functionalization. ${ }^{145,150,176,203,204}$ Commonly used coatings include poly(acrylic) acid, ${ }^{205-208}$ poly(ethylene) glycol (PEG), ${ }^{186,194,195}$ or dextran, ${ }^{209,210}$ all of which are hydrophilic and accepted as biocompatible. ${ }^{145,153,154,158,181,203,211}$ Targeting or therapeutic molecules can then be conjugated to the polymer coating. ${ }^{150,186,192-196}$ Surface ligands are added to NPs through both covalent and non-covalent attachment mechanisms. ${ }^{145}$ Although the type of attachment can influence the biological effects, the preferred mechanism is highly dependent on the ligand being attached and the system in which it will be used. ${ }^{145,212,213}$ Protocols for NP functionalization vary wildly, as many particle-specific factors need to be considered, including stability of the NP, functional groups, conjugation catalyst, solvent choice, and the biomolecule being attached. For multi-functional NPs, hydrophobic drugs can be loaded into the hydrophobic surfactant layer surrounding the NP core..$^{214,215}$

\section{Covalent Attachment}


Covalent ligand attachment is often achieved via 1-ethyl-3-(3-dimethylaminopropyl)carbodiimide (EDC) catalyzed amide bond formation between an amine terminal ligand and an outward facing carboxylic acid on the NP coating. ${ }^{145}$ Carbodiimides activate carboxylate anions to an O-acylurea intermediate in the condensation reaction between amines and carboxylic acids. Then the amine can attack the intermediate to form an amide or another carboxylate can attack to generate an anhydride, which is then attacked by the amine. ${ }^{216}$

EDC is a popular choice for NP modification because it is water soluble, the excess reagent is easy to remove, and the reaction can take place at room temperature. ${ }^{145,217-}$ ${ }^{220}$ However, despite being one of the most common strategies for NP functionalization, the protocols, ${ }^{221,210,222-225}$ and success rates, for EDC-mediated attachments vary significantly. ${ }^{145,218,221,226}$ Further complicating matters, EDC initially gained wide acceptance for its ability to form amide bonds on surfaces, and adaptation of the surface modification protocol for use with NPs in solution requires additional consideration. ${ }^{218,221}$ For example, in many cases excess EDC causes NP aggregation or precipitation, ${ }^{222,145,208}$ possibly because the positively charged activated EDC molecules neutralize the negative charges of the carboxyl groups that are keeping the particles suspended. ${ }^{145}$

The use of EDC for both surface and NP modification requires careful consideration of the reaction conditions. Controlling reaction $\mathrm{pH}$ is critical, as only the protonated form of 
EDC participates in reactions. With a $\mathrm{pK}_{\mathrm{a}}$ of 3.5 , EDC is most active in acidic conditions $(\mathrm{pH}<4) .{ }^{217}$ The $\mathrm{pK}_{\mathrm{a}}$ and ideal reaction $\mathrm{pH}$ for the moieties being linked should also be considered. Once EDC is added to the reaction, timing is also critical as EDC rapidly hydrolyzes in aqueous solutions. ${ }^{216,217}$ Given the proclivity of EDC to hydrolyze in the presence of water, the use of properly stored reagent and freshly prepared EDC solutions is imperative. This could contribute to the reported inconsistencies in EDC efficiency and reaction concentrations. EDC is readily available in large quantities, particularly relative to the amounts needed for small-scale reactions, and the water sensitivity of the reagent would allow for degradation with time, repeated container opening, or improper storage conditions. As a result, larger quantities of reagent would be needed the longer the container is open.

\section{Non-Covalent Attachment}

Non-covalent attachments include electrostatic, hydrophobic, and affinity interactions. ${ }^{145}$ Although non-covalent interactions can be easier to induce than covalent linkages, they are less stable and offer less control of binding ratios and ligand orientation. Even so, hydrophobic interactions are frequently employed to orient polymer coatings on surfactant-coated NPs. When amphiphilic polymers are used, it is assumed that the hydrophobic side chains will associate with the NP surfactant, forcing the polymer backbone to form shell around the NP with the hydrophilic moieties facing outwards. ${ }^{208}$ The hydrophobic surfactant layer can be loaded with hydrophobic drugs, like docetaxel. ${ }^{145,215}$ At the particle surface, the outward facing functional groups of the 
polymer are charged in many aqueous solvents. The reactivity of these groups, typically carboxylic acids or amines, and thus their affinity for interacting with molecules of the opposite charge, can be mediated by adjusting the solvent $\mathrm{pH} .{ }^{145} \mathrm{DNA}$, siRNA, and proteins can be adsorbed to the charged NP surface through electrostatic interactions. 145,198

\subsubsection{SPIONs as Multi-Functional Therapeutics}

SPIONs, which are nano-sized maghemite $\left(\mathrm{\gamma}-\mathrm{Fe}_{2} \mathrm{O}_{3}\right)$ or magnetite $\left(\mathrm{Fe}_{3} \mathrm{O}_{4}\right),{ }^{143,145}$ have been investigated for a variety of biomedical applications, including many multi-functional platforms that exploit the magnetic properties of the particles in addition to their biocompatibility. ${ }^{148,152,154,157,158,227}$ Because SPIONs are superparamagnetic, they are magnetic when placed in a magnetic field, like an MRI, but do not retain that

magnetization when the field is removed. ${ }^{228}$ Their strong magnetization when in a magnetic field allows them to function as MRI contrast agents, while the lack of magnetization in the absence of a magnetic field keeps the SPIONs from agglomerating during storage or circulation. ${ }^{143,145}$

SPIONs have been investigated for a variety of biomedical applications, including roles in drug delivery platforms, ${ }^{152,195,227} \mathrm{MRI}$ contrast agents, ${ }^{148,152,195}$ and magnetic hyperthermia. ${ }^{152,197}$ Ferumoxytol (FeraHeme), a dextran-coated SPION formulation, has received FDA approval for use a treatment for anemia in patients with chronic kidney disease. ${ }^{149,150,157,229}$ While there is some debate surrounding the biocompatibility of bare 
SPIONs, ${ }^{143,154,157,181}$ coated SPIONs are considered biocompatible and are attractive platforms for biomedical technologies. ${ }^{143,150,152,154,181}$ In addition, SPIONs are considered easy to functionalize, making them an ideal base for multi-functional theranostics. ${ }^{152,160,192,195,214}$

\subsection{5a SPION Therapeutics}

SPION therapeutics are generally complex, with multiple layers surrounding the SPION core. Typically the SPION core is coated in a surfactant, like oleic acid, which is then coated with an amphiphilic polymer to improve aqueous solubility and provide reactive sites for further functionalization. ${ }^{145,150}$ The polymer coatings also improve circulation halflife, as bare SPIONs are rapidly cleared, ${ }^{154}$ and enhance biocompatibility. ${ }^{33,154,158,181,211}$ Targeting or therapeutic molecules are then conjugated to the polymer coating. ${ }^{150,186,192-}$ 196 Hydrophobic drugs can be loaded into the hydrophobic surfactant layer surrounding the SPION core. ${ }^{160,185,214,215,230-233}$

Intravenously administered SPIONs, without specific targeting ligands, primarily accumulate in the liver and spleen but can also be found in the heart, lungs, kidney, brain, stomach, small intestine, and bone marrow. ${ }^{152,161,199,234}$ The distribution of SPIONs through the body, including into the brain, demonstrates their ability to penetrate a variety of target tissues when used for drug delivery or diagnostic purposes. SPIONs also accumulate passively in tumors due to leaky vasculature and poor lymph clearance, and 
the addition of targeting ligands can further enhance accumulation in target tissues. ${ }^{150,186,192-196}$

Once SPIONs are taken up by cells, they are metabolized to elemental iron. ${ }^{150,152}$ SPIONs are degraded within lysosomes to elemental iron, which is released to the cytoplasm and handled by normal iron regulatory mechanisms. ${ }^{152,154,235,236}$ There has been some concern that the elemental iron could contribute to target organ toxicity via the generation of ROS through the Fenton reaction or iron accumulation leading to iron overload. ${ }^{143,152}$ In reality, however, the amount of iron introduced from SPION therapeutics is small compared to the normal blood iron stores. ${ }^{154,235,236}$ The success of Ferumoxytol, an FDA-approved SPION-based intravenous treatment for chronic iron deficiency anemia, demonstrates the effective in vivo metabolism of SPIONs and their biocompatibility. ${ }^{149,229}$

\subsection{5b SPIONs as MRI Contrast Agents}

MRI is a sensitive imaging technique that does not involve radiation or radioactive tracer dyes, like PET or CT scans. However, MRI does not have cellular level resolution. Currently gadolinium $(\mathrm{Gd})$ based contrast agents are used to improve resolution, but gadolinium causes severe complications, including nephrotoxicity, in a subset of patients. ${ }^{150}$ SPION-based contrast agents can act as T2-enhancing contrast agents to aid in the identification of lesions, like tumors and metastases, without the toxicity of gadolinium. ${ }^{154,160,161}$ 
SPIONs passively accumulate in the liver, spleen, tumors, and areas of inflammation, and their magnetic properties allow for easy, non-invasive detection of their in vivo distribution with MRI. ${ }^{154} \mathrm{~T} 2$ signal in the liver and spleen is decreased within minutes of intravenous SPION administration. ${ }^{150,154}$ As SPIONs leak out of circulation they drain to the lymphatic system, allowing for lymph node imaging. ${ }^{154}$ Dextran-coated SPIONs can remain in circulation for nearly 2 hours, lending utility as blood-pool contrast agents. ${ }^{154}$ Conjugation of targeting ligands further increases SPION concentration, and thus contrast enhancement, in tumors and target tissues. ${ }^{150,154,192}$ However, the strong magnetic response of uncoated SPIONs can be reduced when organic coatings and surface modifications are present, which should be considered when designing heavily-modified multi-functional SPION platforms. ${ }^{158}$

\subsection{5c SPIONs as Drug Delivery Vehicles}

Systemic administration of drugs typically requires large drug doses to achieve therapeutic concentrations in target tissues. The inability of drugs to accumulate specifically in target tissue is a significant obstacle in cancer treatment because doselimiting systemic adverse effects can occur before therapeutic concentrations are reached. ${ }^{237}$ Delivery systems that direct drugs to, or facilitate drug release in, tumors can improve therapeutic efficacy and reduce off-target effects. SPIONs are biocompatible and passively distribute to tumors in vivo, making them a popular choice for drug delivery platforms. ${ }^{150,152,154,186,192-196}$ SPIONs are also compatible with a variety of coatings and 
functionalization techniques, allowing modifications that can improve targeting, biocompatibility, or deliver multiple drugs at once. ${ }^{152,160,192,195,214}$

Therapeutic agents can be incorporated into SPIONs in a number of ways. ${ }^{192}$ Antisense oligonucleotides (ASO), antibodies, or enzymes are often covalently linked to the outer surface of the particle. ${ }^{150,189,238-240}$ Lipophilic drugs like paclitaxel ${ }^{160,215}$ or doxorubicin ${ }^{185,230-233}$ can be loaded into a surfactant layer between the SPION core and the polymer coating. Drugs can also be attached through links that are readily cleaved in response to changes in $\mathrm{pH}^{230,231,233}$ or tissue-specific enzymes. ${ }^{237}$ Additionally, combinations of drugs can be used to target multiple cancer pathways simultaneously or one agent can be used to sensitive the cancer cells to the other. ${ }^{160}$

SPIONs, and other NPs, functionalized with ASO are able to overcome many of the obstacles that preclude in vivo use of therapeutic oligonucleotides. When the surface of polymer coated SPIONs was decorated with covalently linked SSDNA in vitro cell uptake increased significantly. ${ }^{240}$ While very few polymer-coated SPIONs were internalized, even at high doses, the DNA-bearing SPIONs were taken up in a dose-dependent manner. This is consistent with reports that gold NPs functionalized with ASO are internalized in significantly larger quantities than non-functionalized or peptide-functionalized controls ${ }^{241}$ and can decrease target protein expression more efficiently than commercially available transfection methods. ${ }^{213}$ ASO loading onto the gold NPs is more easily controlled than loading onto SPIONs, and this precise control allows for comparison of the uptake and protein regulatory effects of NPs with varied ASO densities. NPs with more densely 
packed ASO were taken up at higher rates than those with less ASO per NP. ${ }^{213,241}$ Similar ASO-gold NP systems reach glioblastomas in vivo and sensitize the tumor cells to apoptotic stimulants; this system is now being testing in phase I clinical trials. ${ }^{242}$ Although many of the proof-of-concept studies for ASO-NP systems have been carried out using gold NP cores, it is believed that the core material is of little significance. ${ }^{34}$ Rather, the dense oligonucleotide packing on the surface of the NP drives the biological effects.

\subsection{5d Multi-functional SPION Systems}

Although the SPION-based agents currently on the market each serve a single purpose (iron replacement or providing MRI contrast), many of the research-stage formulations are multi-functional. ${ }^{192}$ Carefully engineered systems can serve, simultaneously, as drug delivery platforms and MRI contrast agents. ${ }^{150,152,215,227,232,233}$ Other multi-functional systems aim to sensitize tumor cells to a drug carried within the platform. ${ }^{201}$ When targeting molecules are used in conjunction with the aforementioned strategies, SPIONs can be used to label or track tumor cells with specific features. The ability to label specific cells, combined with the strong magnetic response of SPIONs, allows for identification of small metastases using MRI instead of radiation-based imaging. ${ }^{150,230,237}$ Potential for drug resistance could also be identified without the need for a tumor biopsy, as characteristic cell surface markers like Her2 or PSMA can be targeted with SPIONantibody conjugates. ${ }^{201,202}$ 


\subsection{Carbon Nanotubes}

\subsubsection{Carbon Nanotubes}

Carbon nanotubes (CNTs) are hollow tubes formed from rolled up graphene sheets. ${ }^{177}$ Single sheets of graphene form single-wall carbon CNTs (SWCNTs) while multi-wall CNTs (MWCNTs) have multiple, concentric graphene layers. ${ }^{142}$ CNTs of various lengths, widths, and surface chemistry can be obtained by varying synthesis methods. ${ }^{177}$ As CNTs are considered highly amenable to functionalization, further variety can be achieved through surface modifications. Pristine and functionalized CNTs possess unique physical and chemical properties that make them of interest to scientists, engineers, and industry. Specifically, their light weight, high tensile strength, thermal and chemical stability, and conductivity have led to the incorporation of CNTs into electrodes, lithium ion batteries, thin film flexible electrodes, and sports equipment. ${ }^{142,143,164,177}$ The use of CNTs in biomedical applications, including use in drug and gene delivery, ${ }^{243-245}$ vaccinations, ${ }^{246}$ biosensors, ${ }^{164,247}$ and tissue scaffolding, ${ }^{142,248}$ has been a popular research endeavor. As CNTs find their way into an increasing variety of consumer products, concerns have arisen about their toxicity. ${ }^{171,249-251}$ In fact, concerns about CNT toxicity are considered a limiting factor for their use in biomedical applications. ${ }^{147,177}$

\subsubsection{Carbon Nanotube Toxicity}

The integration of CNT in an increasing number of consumer goods creates a high likelihood of industrial exposures. Because CNTs are small, light, and easily aerosolized, 
inhalation exposures are a primary concern. ${ }^{141,252}$ The toxicity of CNTs depends heavily on the particle physical properties, dose, exposure route, and exposure time. However, there is little standardization of these variables, or their reporting, across studies from different groups. ${ }^{163,251}$ Despite conflicting reports, it is generally agreed that CNTs are toxic/harmful to cells, although the degree of damage depends on physical characteristics including residual catalysts, aggregation state, surface chemistry, and structural differences. ${ }^{142,164,171,253}$

A lack of standardization in NP characterization, dose determination, and toxicity assessment has contributed to conflicting toxicity reports. ${ }^{164,165}$ As variations in CNT size, shape, surface chemistry, aggregation state, synthesis methods, and dispersal techniques all affect CNT toxicity, inconsistencies in these variables have made it difficult to compare toxicity studies performed by different groups. ${ }^{142,143,171,253}$ However, at this point it is largely accepted that CNTs are hazardous to human health, and the conversation has shifted towards determining the specific effects and elucidating the mechanisms and time course of the damage, as well as the influence of various particle properties. ${ }^{156,252,254}$

Many initial toxicity studies were conducted with high-dose, short-term CNT exposures, which are poor models of real world conditions. ${ }^{171,250}$ Low dose, chronic exposure models are needed to simulate most likely exposure conditions. CNTs are small, light, and easily aerosolized, making occupational inhalation exposures are considered the most likely real world exposure route. ${ }^{165,252}$ In addition, CNTs have been 
likened to asbestos in terms of their fibrous shape, bio-persistence, and ability to induce pulmonary inflammation and fibrosis. ${ }^{164,171,249,250,252}$ Miscalculation of the long-term risks of asbestos had devastating human health and financial implications. Unfortunately the rapid incorporation of CNTs into consumer goods, without a full understanding of the human health risks, may lead to down the same path. ${ }^{253}$ Thus, improved understanding of CNT toxicity is needed to remediate the introduction of a potential health hazard.

Acutely, inhaled CNTs cause pulmonary irritation, inflammation, and fibrosis. ${ }^{170,171,175,251,255}$ However, CNT bio-persistence allows for accumulation over time. ${ }^{165,174,256,257}$ CNT accumulation can cause direct and indirect physical and chemical damage to bronchial epithelial cells and pulmonary macrophages and the full extent of the progressive damage, particularly to DNA, can take years, or decades, to manifest. ${ }^{171,174,175,250}$ Asbestos-related malignancies are often detected 30-40 years following exposure. ${ }^{258}$ Given their physical similarities, it is reasonable to extrapolate that CNT-related damage could occur over a similar time frame. Despite the likely health risks, there are currently few studies investigating the long-term effects of chronic, low-dose CNT exposures, although chronic CNT exposure has been shown to induce malignant transformation of lung epithelial cells in vitro ${ }^{172,259}$ and promote carcinogenesis in vivo. ${ }^{174,252}$

\subsubsection{Role of CNT Physical Properties in Toxicity}

CNT structure (length, width, and aspect ratio), functionalization, and aggregation state are strong determinants of toxicity. ${ }^{248,165}$ The size of inhaled particulates determines 
how deep into the lung they are able to penetrate. ${ }^{248}$ Particles $<100 \mathrm{~nm}$ bypass most of the lungs defense mechanisms and deposit at the broncho-alveolar junctions and in the alveoli themselves. ${ }^{141,165,175,174}$ After CNTs have deposited, normal lung clearance mechanisms are evoked. However, the high aspect ratio of CNTs impedes phagocytosis by macrophages. ${ }^{164,260}$ Consequently, CNTs can persist in the lungs for up to 11 months. ${ }^{141,165,175,174}$ The persistent presence of foreign material stimulates an ongoing inflammatory response that can ultimately lead to granulomas, fibrosis, or carcinogenesis. ${ }^{164,260}$ CNT shape affects the extent of the damage. Longer, thinner CNTs are better able to penetrate lung cells and induce more granuloma formation and inflammation than shorter or wider CNTs. ${ }^{164,248,260}$ Agglomeration changes the shape, surface, and stiffness of the particles. ${ }^{143,164}$ Some studies have found that SWCNT bundles are less toxic than single SWCNTs, ${ }^{143}$ but others have reported that the increased stiffness of CNT aggregates resulted in greater cytotoxicity than dispersed CNTs. ${ }^{164}$

CNT surface modifications can affect the size, surface charge, and biological activity of the particle. ${ }^{164}$ Pristine (non-functionalized) CNTs, which are primarily graphene (carbon) are hydrophobic and highly reactive. ${ }^{248,261}$ When CNTs are designed for biomedical applications, functionalization improves biocompatibility by reducing CNT immunogenicity and surface reactivity while increasing aqueous solubility. ${ }^{177,143,164,248,178}$ Solubilized CNTs can be further decorated with therapeutic or targeting ligands, including antibodies, biomolecules, or nucleic acids. ${ }^{164,245,246,261}$ 
Reports of functionalized CNTs being biocompatible are carried out in different conditions than studies looking at toxicity from inadvertent exposures. One major difference is that functionalized CNTs for therapeutic applications are injected intravenously, resulting in different distribution, immune reactivity, and clearance mechanisms than those that drive pulmonary toxicity. ${ }^{178,183,262}$ Another difference is that therapeutic CNTs are more likely to have a narrow size distribution and to be uniformly suspended with few agglomerates. ${ }^{178,183}$ Additionally, evaluation of many of the therapeutic CNT platforms seems to primarily rely on short exposure times and fewer toxicity endpoints. ${ }^{143,183}$ These short (4-48 hours) exposure times may be relevant to the expected delivery to the platform, but the CNTs will likely persist in vivo and can have effects beyond those time points.

CNT functionalization for therapeutic applications necessitates dosing considerations similar to those discussed in 1.1.6 Dosing NPs for Toxicity Evaluations. The use of pristine CNTs, which have markedly different physical and chemical properties than the functionalized system, as a control introduces additional confounding factors. Particularly for in vitro testing, where the system is stationary and stagnant, the effects of improved solubility of the functionalized CNTs alone can contribute heavily to the decrease in observed toxicity.

For example, improved biocompatibility of glycodendrimer-coated SWCNTs, versus pristine SWCNTs, has been reported. ${ }^{183}$ However, the improvements noted in vitro may not be as significant as they appear due to differences in the way the particles interact 
with the in vitro system. The coated SWCNTs reportedly stay homogenously suspended in aqueous solutions for many months, while the pristine SWCNT control precipitates almost immediately. In an in vitro test system with adherent cells on the bottom of the culture dish, many more of the rapidly precipitating pristine SWCNTs will be available to interact with the cells than the stably suspended coated particles. ${ }^{165}$

In addition, cells were treated with equivalent masses of the coated and uncoated CNTs. ${ }^{183}$ Although the contribution of the coating to the particle mass was not reported, enough coating to confer stable suspension in aqueous solution would be expected to have a measureable contribution. Even if the coating was a small percentage of the total mass, there would inherently be fewer glycodendrimer-coated SWCNTs in a given mass than there would be in an equivalent mass of pristine SWCNTs. If concentration dependent effects are expected, adding different absolute numbers of particles negates the validity of the mass-equivalency dosing. The extreme differences in particle solubility would also change the dose the cells were ultimately exposed to.

\subsubsection{Modeling Real World Exposures}

CNTs are small, low-density particles are easily aerosolized. Thus, occupational inhalation exposures are the most pressing public health concern. ${ }^{141,252,165}$ However, the CNTs used for industrial applications are heterogeneous mixture of particle sizes, shapes, and agglomeration states. ${ }^{166}$ Approximating the composition of real world exposures is often challenging when designing toxicity assessments, and many evaluations instead utilize more homogeneous mixtures. Modeling the dose of real world CNT exposures for 
in vitro or in vivo studies is also difficult. ${ }^{141,165}$ Following inhalation, CNTs deposit in small airways and alveoli, where they can remain for upwards of 11 months. ${ }^{141,165,175,174}$ Normal lung clearance mechanisms are invoked but are largely ineffective at degrading the CNTs. ${ }^{141,175,174}$ Consequently, the CNTs accumulate over time despite the low concentration of each individual exposure. In experimental systems, doses for are often calculated to (model) a lifetimes worth of exposures. ${ }^{166}$ This has led to the use of extraordinarily high bolus doses in both in vitro and in vivo studies. ${ }^{166}$ Acute toxicity is readily apparent when these large doses are applied, which has contributed to the continued use of unreasonable doses. ${ }^{165}$ In contrast, lower, more realistic doses induce much less overt toxicity in acute evaluations. ${ }^{166}$ The validity of life-time exposure dosing for assessing chronic toxicity has been questioned. ${ }^{165,166}$ Chronic in vitro CNT exposure models, in which cells are cultured with sub-acute concentrations of CNTs for 6 months, have been developed to overcome this limitation. ${ }^{172,263}$ 


\subsubsection{Acute Toxicity of CNTs}

Acute exposure to CNTs results in dose- and time-dependent decreases in cell viability in vitro ${ }^{143,260}$ and inflammation and fibrosis in in vivo inhalation models. ${ }^{260}$ CNTs are internalized within 48 hours of exposure in vitro, ${ }^{260}$ which induces oxidative stress, release of IL- 8 , and overexpression of immune and inflammatory genes. ${ }^{143}$ Genotoxicity following acute CNT exposures, including aneuploidy, centrosome fragmentation, damage to mitotic spindles, and increased numbers micronuclei, has also been reported. $174,256,264$

\subsubsection{Chronic Toxicity of CNTs}

Health problems stemming from chronic occupational exposure to CNTs could take years, or decades, to become apparent, similar to asbestos induced mesothelioma, and short-term, high dose studies do not provide a full picture of the risks. ${ }^{171,250,175,258}$ The long latent period between exposure and disease onset can make it difficult to assess the potential carcinogenicity and carcinogenic mechanisms. ${ }^{174,260}$ Consequently, acute, highdose exposures are more widely studied even though they are not representative of most real world exposures. ${ }^{171,250}$ Over time, the particles cause continual inflammation, irritation, and immune system activation. In addition to the damage inflicted through physical interactions of the particles with cells, organelles, and genetic material, the ongoing inflammatory response also triggers changes in cell behavior. $170,255,174,256,264$ Chronic inflammation is, by itself, a risk factor for the development of cancer, as evidenced by the increased risk of malignancy in patients with autoimmune diseases. 174,256 Acutely, CNT genotoxic effects include the induction of aneuploidy, centrosome fragmentation, damage to mitotic spindles, and increased numbers micronuclei. ${ }^{174,256,264}$ 
Genetic damage accumulates over time and the impacts become evident long after the inciting event. 250,258 CNT exposure in a mouse inhalation model resulted in K-ras oncogene mutations. ${ }^{255,256}$ Despite the evidence for CNT-induced DNA damage, their status as an oncogenic initiator is still unconfirmed. ${ }^{175}$ However, a murine inhalation study, using an initiator/promotor model, demonstrated that CNTs do act as tumor promotors. ${ }^{252}$

In vitro chronic exposure models have been developed to better understand the effects of long-term, low dose CNT exposures. ${ }^{250,175,172,259,265}$ Many of these studies have examined the response of non-cancerous bronchial epithelial cells, which are the first layer of defense against inhaled CNTs in humans. ${ }^{172,266}$ When lung epithelial cells were exposed to sub-acute doses of CNTs for 6 months, they underwent malignant transformation, with obvious morphological changes appearing half way through the exposure period. Following the CNT exposure, the transformed lung epithelial cells proliferated more rapidly, were more invasive, demonstrated anchorage-independent growth, and were resistant to apoptosis. ${ }^{175,172,259}$ CNT-induced cell cycle dysfunction has been linked to decreases in p53 expression, ${ }^{267}$ while apoptosis resistance has been linked to increases in C-FLIP expression. ${ }^{268}$ Increases in transformed cell invasion and migration have been related to increases in matrix metalloproteinase (MMP) expression. ${ }^{259}$ 


\subsection{Figures and Tables}

\subsubsection{Figures}

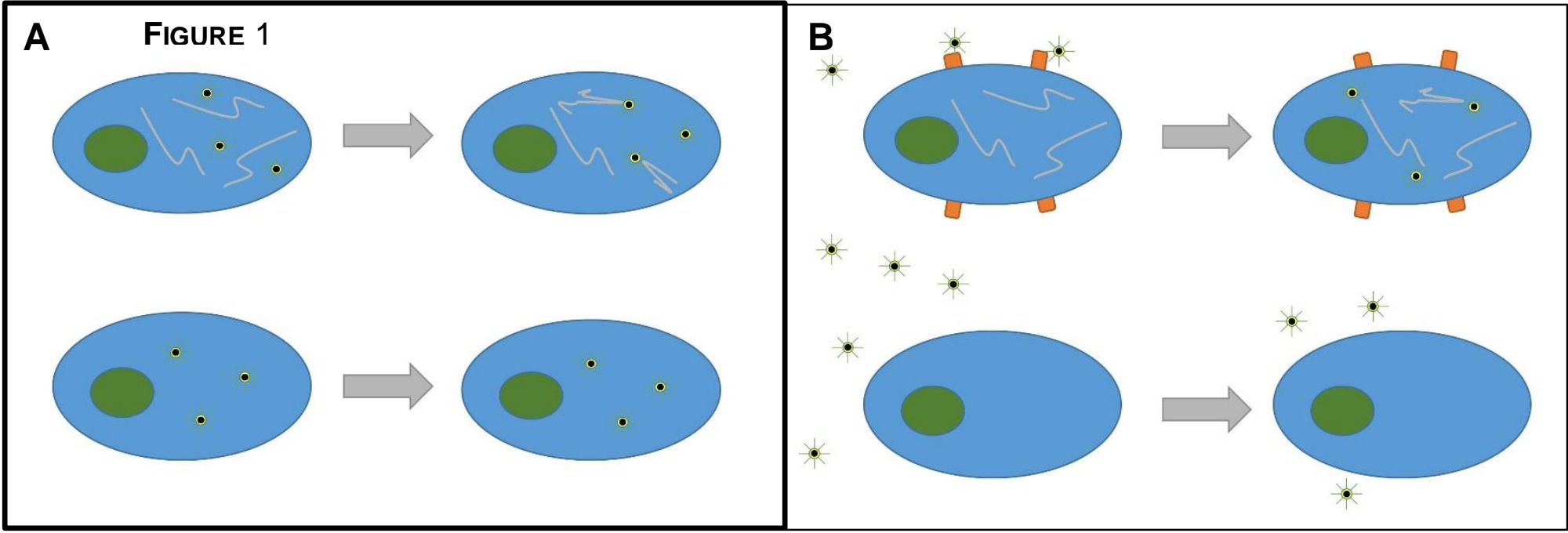

Figure 1. Specificity of NPs, in the form tissue specific effects, can be conferred through two distinct mechanisms. (A) NPs that target a cell- or tissue specific pathway will be taken up by all cells but only effect cells that have the target feature. For the purposes of demonstration, the illustration shows NPs bearing antisense oligonucleotides that target a cancer specific protein. (B) NPs that recognize surface features of target cells will be internalized with greater specificity. Antibodies targeting cell surface features can be used to improve uptake specificity, as can molecules that are readily internalized, including folate. 
Figure 2
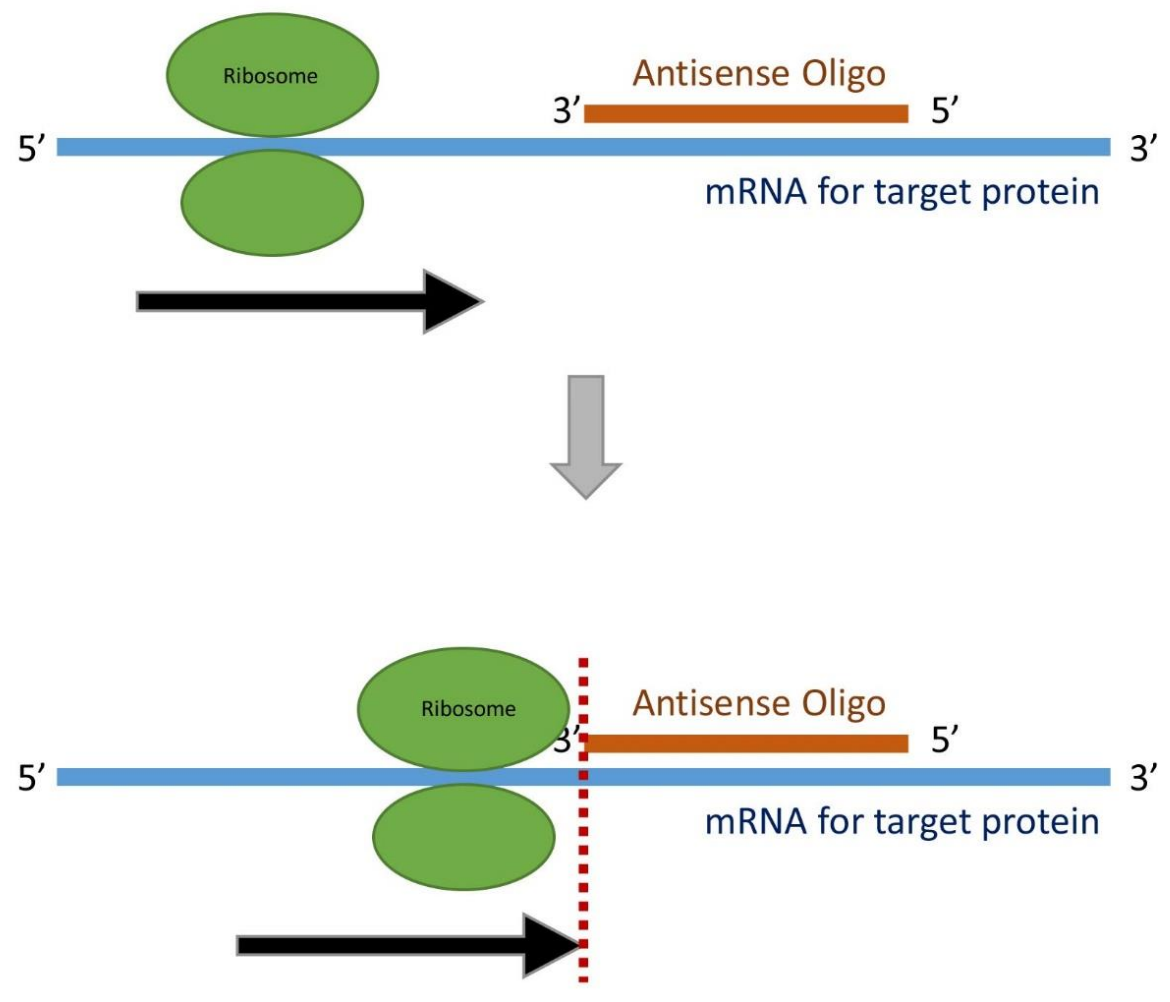

Figure 2. Mechanism of ASO protein synthesis inhibition. Binding of the ASO to the complementary sequence of the target protein mRNA occurs in the cytoplasm. Ribosomal translocation along the mRNA is physically blocked by ASO hybridization with its complementary sequence. 
Figure 3

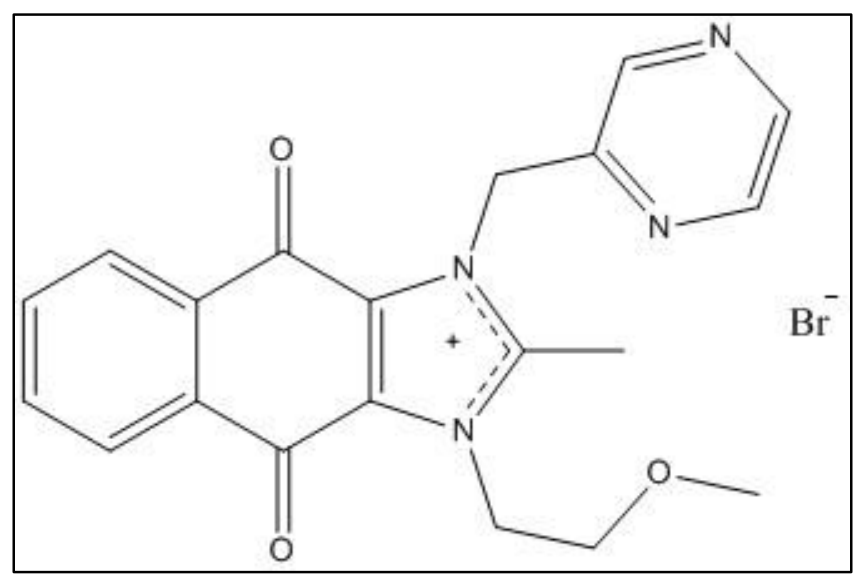

Figure 3. Structure of YM155 (1-(2-Methoxyethyl)-2-methyl-4,9-dioxo-3-(pyrazin-2ylmethyl)-4,9-dihydro-1 $H$-naphtho[2,3-d] imidazolium bromide), a small molecule inhibitor of survivin. 


\subsubsection{Tables}

Table 1. Survivin expression in human tumor samples.

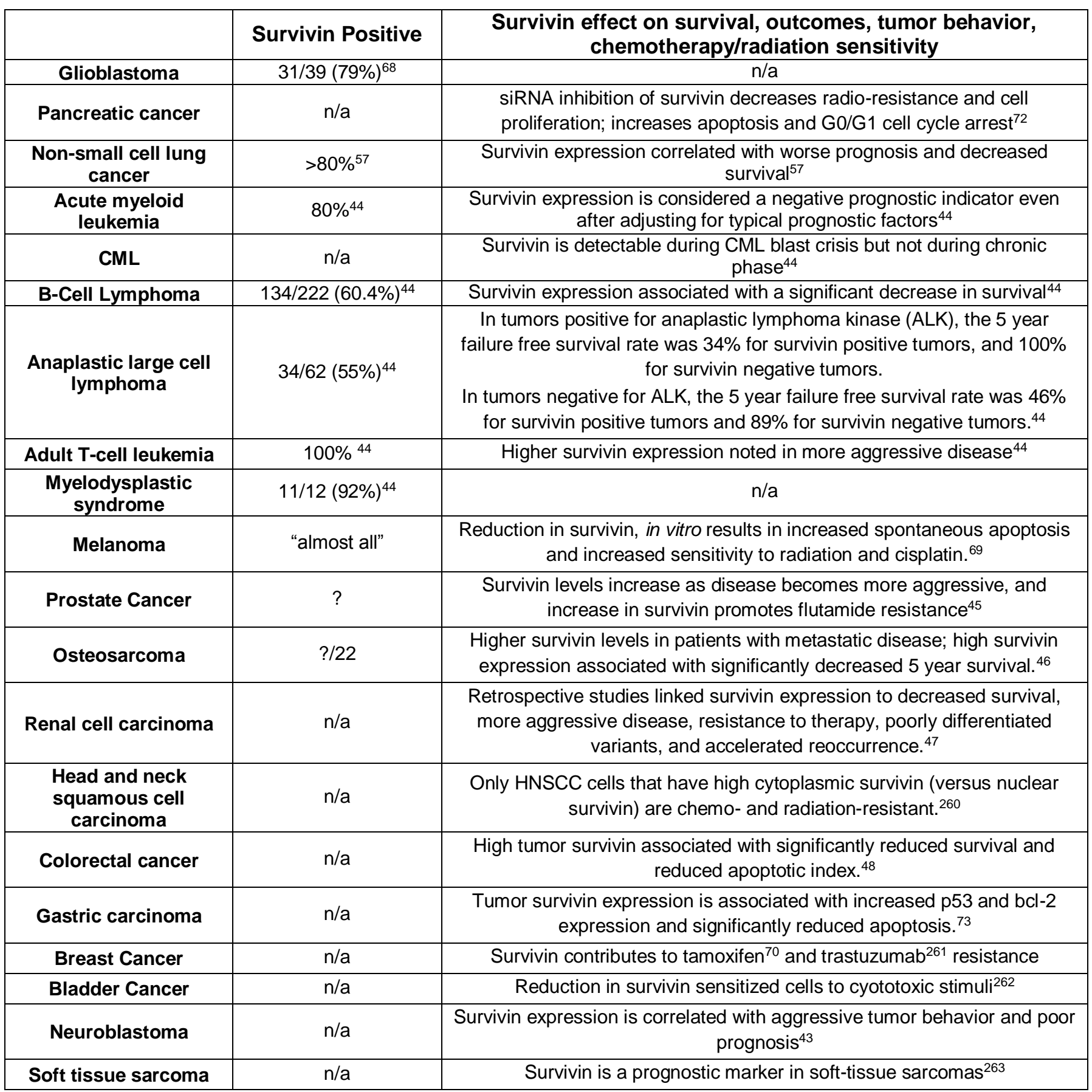


Table 2. Interference of NPs with in vitro assessments of toxicity

\begin{tabular}{|c|c|c|c|}
\hline & Techniques/Reagents & Assay Mechanism & Usage Considerations \\
\hline $\begin{array}{c}\text { Cell } \\
\text { Proliferation } \\
143,155,162,203\end{array}$ & $\begin{array}{l}\text { MTT (3-(4,5-dimethylthiazol-2- } \\
\text { yl)-2,5-diphenyl tetrazolium } \\
\text { bromide) } \\
\text { WST-1 (a water soluble } \\
\text { tetrazolium salt) } \\
\text { MTS (3-(4,5-dimethylthia- zol-2- } \\
\text { yl)-5-(3-carboxymethoxyphenyl) } \\
\text { 2-(4-sulfophenyl)- 2H- } \\
\text { tetrazolium) }\end{array}$ & $\begin{array}{l}\text { Colorimetric determination of } \\
\text { mitochondrial viability } \\
\text { Conversion of a tetrazolium salt to a } \\
\text { formazan dye, resulting in a color } \\
\text { change proportional to the number of } \\
\text { metabolically active cells }\end{array}$ & $\begin{array}{l}\text { Fast, reproducible results }{ }^{155} \\
\text { Interference from NPs: } \\
\text { - } \quad \text { Direct reactions of CNT, porous silica, and carbon black with tetrazolium } \\
\text { salts - falsely increases measured viability }{ }^{142,155,156,264} \\
\text { - } \quad \text { Adsorption of formazan dye to CNT - measured viability is falsely } \\
\text { low }{ }^{142,155,163} \\
\text { - Spontaneous activation of MTT by graphene }{ }^{142,265} \\
\text { - } \quad \text { NP can physically block light transmission }{ }^{144}\end{array}$ \\
\hline $\begin{array}{l}\text { Membrane } \\
\text { Integrity }\end{array}$ & $\begin{array}{l}\text { Lactate dehydrogenase }(\mathrm{LDH}) \\
\text { release }\end{array}$ & $\begin{array}{l}\text { LDH is an intracellular enzyme that } \\
\text { leaks into the culture media when cell } \\
\text { membrane integrity is compromised. } \\
\text { LDH in media oxidizes lactate to } \\
\text { pyruvate, which triggers the } \\
\text { conversion of a tetrazolium salt to a } \\
\text { formazan dye. }\end{array}$ & $\begin{array}{l}\text { LDH has a half-life of } 9 \text { hours in media; timing of cell death relative to assay } \\
\text { influences results. }{ }^{156} \\
\text { Interference from NPs: } \\
\text { - } \quad \text { LDH can be inactivated by } \mathrm{Cu} \text { or silver NPs or adsorb to } \mathrm{TiO}_{2}{ }^{142,266} \\
\text { - } \mathrm{LDH} \text { is deactivated under low } \mathrm{pH} \text { conditions, while a high } \mathrm{pH} \text { stabilizes } \\
\text { the substrate }{ }^{144} \\
\text { - Metal ions can interfere with LDH assay, although SWCNT do not appear } \\
\text { to interfere }{ }^{144,267}\end{array}$ \\
\hline Apoptosis & Caspase activation $^{144,155}$ & $\begin{array}{l}\text { Caspase-3 activation leads to cell } \\
\text { death }\end{array}$ & $\begin{array}{l}\text { Caspase- } 3 \text { is inhibited by trace metal ions, but is mostly unaffected by } \\
\text { changes in } \mathrm{pH}^{144}\end{array}$ \\
\hline $\begin{array}{l}\text { NP } \\
\text { Intracellular } \\
\text { Localization }\end{array}$ & $\begin{array}{l}\text { Transmission electron } \\
\text { microscopy (TEM) }{ }^{142,155}\end{array}$ & $\begin{array}{l}\text { Visualization of electron dense } \\
\text { materials, like metal NPs, and stained } \\
\text { cellular structures allows for } \\
\text { determination of precise intracellular } \\
\text { localization }\end{array}$ & $\begin{array}{l}\text { Does not show organic coatings on NPs, so can only be used to determine } \\
\text { location of NP core } \\
\text { Staining biological samples can introduce electron-dense artifacts that are } \\
\text { similar in size to NPs }{ }^{155}\end{array}$ \\
\hline \multirow[t]{2}{*}{$\begin{array}{l}\text { Quantification } \\
\text { of NP Uptake }\end{array}$} & $\begin{array}{l}\text { Inductively Coupled Plasma } \\
\left(\text { ICP) }{ }^{142,155,162}\right. \\
\text { Colorimetric quantification of iron } \\
\text { (Prussian blue, } 268,269 \\
\text { ferrozine, }{ }^{187,270} \text { - } \\
\text { phenanthroline) }{ }^{271,272}\end{array}$ & $\begin{array}{l}\text { Quantitative measure of metals, with } \\
\text { sub-ppb limits of detection } \\
\text { Reaction of iron with various reagents } \\
\text { allows for colorimetric quantification of } \\
\text { iron concentration using UV-vis }\end{array}$ & $\begin{array}{l}\text { Destructive technique requires acid digestion of samples } \\
\text { Detects metal from physiological sources as well as from treatment } \\
\text { Not useful for carbon-based NPs because of large carbon background in } \\
\text { biological samples } \\
\text { No insight into NP localization }\end{array}$ \\
\hline & Fluorescence Spectroscopy ${ }^{142,155}$ & $\begin{array}{l}\text { Quantitative and qualitative } \\
\text { assessment of the uptake and } \\
\text { intracellular localization of } \\
\text { fluorescently-tagged NPs }\end{array}$ & $\begin{array}{l}\text { Fluorescent dyes alter NP surface properties and can affect uptake and } \\
\text { localization }\end{array}$ \\
\hline
\end{tabular}




\subsection{References}

1. Neesse, A., Krug, S., Gress, T. M., Tuveson, D. A. \& Michl, P. Emerging concepts in pancreatic cancer medicine: targeting the tumor stroma. Onco. Targets. Ther. 7, 33-43 (2014).

2. Hanahan, D. \& Weinberg, R. a. Hallmarks of cancer: the next generation. Cell 144, 646-74 (2011).

3. Engers, R. \& Gabbert, H. E. Mechanisms of tumor metastasis: cell biological aspects and clinical implications. J. Cancer Res. Clin. Oncol. 126, 682-692 (2000).

4. Rasmussen, L. \& Arvin, A. Chemotherapy-Induced Immunosuppression. Environ. Health Perspect. 43, 21-25 (1982).

5. Dimeo, B. F., Fetscher, S., Lange, W., Mertelsmann, R. \& Keul, J. Effects of Aerobic Exercise on the Physical Performance and Incidence of TreatmentRelated Complications After High-Dose Chemotherapy. Blood 9, 3390-3394 (2016).

6. Cf, J. \& B, F. Impaired Thinking in Patients with Breast Cancer and Depression. J. Palliat. Care Med. 06, 1-7 (2016).

7. Spiegel, D. Mind matters in cancer survival. Psychooncology. 593, 588-593 (2012). 
8. Kissane, D. W. Marriage is as protective as chemotherapy in cancer care. J. Clin. Oncol. 31, 3852-3 (2013).

9. Chow, R. et al. Enteral and parenteral nutrition in cancer patients: a systematic review and meta-analysis. Ann. Palliat. Med. 5, 30-41 (2016).

10. Cameron, A. C., Touyz, R. M. \& Lang, N. N. Vascular Complications of Cancer Chemotherapy. Can. J. Cardiol. (2015). doi:10.1016/j.cjca.2015.12.023

11. Ang, C. W. et al. The most frequent cause of 90-day unplanned hospital readmission following colorectal cancer resection is chemotherapy complications. Color. Dis. 17, 779-86 (2015).

12. Macdonald, T. M., Mackenzie, I. S., Wei, L., Hawkey, C. J. \& Ford, I. Methodology of a large prospective, randomised, open, blinded endpoint streamlined safety study of celecoxib versus traditional non-steroidal antiinflammatory drugs in patients with osteoarthritis or rheumatoid arthritis: protocol of the standard of ca. BMJ Open 3, 1-8 (2013).

13. Westphalen, S. \& Kavanagh, J. GnRH antagonists in the treatment of gynecological and breast cancers. Endocr. Relat. Cancer 10, 291-299 (2003).

14. Cook, T. \& Sheridan, W. P. Development of GnRH Antagonists for Prostate Cancer: New Approaches to Treatment. Oncologist 5, 162-168 (2000). 
15. Ezaki, T. et al. Efficacy of treatment with a $\mathrm{GnRH}$ antagonist in prostate cancer patients previously treated with a GnRH agonist. Cancer Chemother. Pharmacol. 76, 301-6 (2015).

16. Shiau, A. K. et al. The Structural Basis of Estrogen Receptor / Coactivator Recognition and the Antagonism of This Interaction by Tamoxifen. Cell 95, 927-937 (1998).

17. Group, T. B. I. G. (BIG) 1-98 C. A Comparison of Letrozole and Tamoxifen in Postmenopausal Women with Early Breast Cancer. N. Engl. J. Med. 2747-2757 (2005).

18. Kobayashi, K. \& Hagiwara, K. Epidermal growth factor receptor (EGFR) mutation and personalized therapy in advanced nonsmall cell lung cancer (NSCLC). Target. Oncol. 8, 27-33 (2013).

19. Mok, T. S. et al. Gefitinib or Carboplatin-Paclitaxel in Pulmonary Adenocarcinoma. N. Engl. J. Med. 361, 947-957 (2009).

20. Zhou, C. et al. Erlotinib versus chemotherapy as first-line treatment for patients with advanced EGFR mutation-positive non-small-cell lung cancer (OPTIMAL, CTONG-0802): a multicentre, open-label, randomised, phase 3 study. Lancet. Oncol. 12, 735-42 (2011). 
21. Gathmann, I. et al. Imatinib Compared with Interferon and Low-Dose Cytarabine for Newly Diagnosed Chronic-Phase Chronic Myeloid Leukemia. N. Engl. J. Med. 348, 994-1004 (2003).

22. Gathmann, I. et al. Five-Year Follow-up of Patients Receiving Imatinib for Chronic Myeloid Leukemia. N. Engl. J. Med. 355, 2408-2417 (2006).

23. Kantarijian, H. et al. Hematologic and Cytogenic Responses to Imatinib Mesylate in Chronic Myelogenous Leukemia. N. Engl. J. Med. 346, 645-652 (2002).

24. Kunze, D., Kraemer, K. \& Fuessel, S. Antisense Oligonucleotides: Insights from Preclinical Studies and Clinical Trials. RNA Technol. 285-303 (2010). doi:10.1007/978-3-642-12168-5

25. Kim, D. H. \& Rossi, J. J. Strategies for silencing human disease using RNA interference. Nat. Rev. Genet. 8, 173-84 (2007).

26. Toth, P. P. Antisense therapy and emerging applications for the management of dyslipidemia. J. Clin. Lipidol. 5, 441-9 (2011).

27. Inoue, H., Hayase, Y., Iwai, S. \& Ohtsuka, E. Sequence-dependent hydrolysis of RNA using modified oligonucleotide splints and RNase $\mathrm{H}$. FEBS Lett. 215, 327-30 (1987). 
28. Walder, R. Y. \& Walder, J. a. Role of RNase H in hybrid-arrested translation by antisense oligonucleotides. Proc. Natl. Acad. Sci. U. S. A. 85, 5011-5 (1988).

29. Bonham, M. a et al. An assessment of the antisense properties of RNase $\mathrm{H}$ competent and steric-blocking oligomers. Nucleic Acids Res. 23, 1197-203 (1995).

30. Nakamura, H. et al. How does RNase H recognize a DNA.RNA hybrid? Proc. Natl. Acad. Sci. U. S. A. 88, 11535-9 (1991).

31. Kanasty, R., Dorkin, J. R., Vegas, A. \& Anderson, D. Delivery materials for siRNA therapeutics. Nat. Mater. 12, 967-77 (2013).

32. Marafini, I. et al. Antisense approach to inflammatory bowel disease: prospects and challenges. Drugs 75, 723-30 (2015).

33. Tiwari, S. B., Ph, D., Amiji, M. M. \& Ph, D. Long-Circulating Polymeric Nanovectors for Tumor-Selective Gene Delivery. Technol. Cancer Res. Treat. 4, 615-625 (2005).

34. Cutler, J. I., Auyeung, E. \& Mirkin, C. a. Spherical nucleic acids. J. Am. Chem. Soc. 134, 1376-91 (2012).

35. Li, S.-D., Chen, Y.-C., Hackett, M. J. \& Huang, L. Tumor-targeted delivery of siRNA by self-assembled nanoparticles. Mol. Ther. 16, 163-9 (2008). 
36. Seferos, D. S., Giljohann, D. A., Rosi, N. L. \& Mirkin, C. A. Locked nucleic acid-nanoparticle conjugates. Chembiochem 8, 1230-2 (2007).

37. Monteleone, G. et al. Mongersen, an oral SMAD7 antisense oligonucleotide, and Crohn's disease. N. Engl. J. Med. 372, 1104-13 (2015).

38. Steele, F. Marking milestones. Nucleic Acid Ther. 23, 243 (2013).

39. Perry, C. M. \& Barman Balfour, J. a. Fomivirsen. Drugs 57, 375-380 (1999).

40. Akdim, F. et al. Efficacy and safety of mipomersen, an antisense inhibitor of apolipoprotein B, in hypercholesterolemic subjects receiving stable statin therapy. J. Am. Coll. Cardiol. 55, 1611-8 (2010).

41. Mita, A. C., Mita, M. M., Nawrocki, S. T. \& Giles, F. J. Survivin: key regulator of mitosis and apoptosis and novel target for cancer therapeutics. Clin. Cancer Res. 14, 5000-5 (2008).

42. Olie, R. A., Simões-wüst, A. P., Baumann, B. \& Simo, A. P. A Novel Antisense Oligonucleotide Targeting Survivin Expression Induces Apoptosis and Sensitizes Lung Cancer Cells to Chemotherapy. Cancer Res. 60, 2805$2809(2000)$.

43. Sasaki, T., Lopes, M. B. S., Hankins, G. R. \& Helm, G. a. Expression of survivin, an inhibitor of apoptosis protein, in tumors of the nervous system. Acta Neuropathol. 104, 105-9 (2002). 
44. Cong, X. L. \& Han, Z. C. Survivin and Leukemia. Int. J. Hematol. 80, 232238 (2004).

45. Zhang, M., Latham, D. E., Delaney, M. a \& Chakravarti, A. Survivin mediates resistance to antiandrogen therapy in prostate cancer. Oncogene 24, 2474$82(2005)$.

46. Osaka, E. et al. Survivin as a prognostic factor for osteosarcoma patients. Acta Histochem. Cytochem. 39, 95-100 (2006).

47. Zamparese, R. et al. Survivin expression in renal cell carcinoma. Cancer Invest. 26, 929-35 (2008).

48. Kawasaki, H., Altieri, D. C. \& Lu, C. Inhibition of Apoptosis by Survivin Predicts Shorter Survival Rates in Colorectal Cancer. Cancer Res. 58, 5071-5074 (1998).

49. Hansen, J. B. et al. SPC3042: a proapoptotic survivin inhibitor. Mol. Cancer Ther. 7, 2736-45 (2008).

50. Pastan, I. \& Hassan, R. Discovery of mesothelin and exploiting it as a target for immunotherapy. Cancer Res. 74, 2907-12 (2014).

51. Tang, Z., Qian, M. \& Ho, M. The role of mesothelin in tumor progression and targeted therapy. Anticancer Agents Med Chem 13, 276-280 (2013). 
52. Kachala, S. S. et al. Mesothelin overexpression is a marker of tumor aggressiveness and is associated with reduced recurrence-free and overall survival in early-stage lung adenocarcinoma. Clin. cancer Res. 20, 1020-8 (2014).

53. Li, M. et al. Mesothelin is a malignant factor and therapeutic vaccine target for pancreatic cancer. Mol. Cancer Ther. 7, 286-96 (2008).

54. Ho, M. et al. Mesothelin expression in human lung cancer. Clin. cancer Res. 13, 1571-5 (2007).

55. Talbot, D. C. et al. Tumor survivin is downregulated by the antisense oligonucleotide LY2181308: a proof-of-concept, first-in-human dose study. Clin. Cancer Res. 16, 6150-8 (2010).

56. Wobser, M. et al. Complete remission of liver metastasis of pancreatic cancer under vaccination with a HLA-A2 restricted peptide derived from the universal tumor antigen survivin. Cancer Immunol. Immunother. 55, 1294-8 (2006).

57. Giaccone, G. et al. Multicenter phase II trial of YM155, a small-molecule suppressor of survivin, in patients with advanced, refractory, non-small-cell lung cancer. J. Clin. Oncol. 27, 4481-6 (2009).

58. Natale, R. et al. Evaluation of antitumor activity using change in tumor size of the survivin antisense oligonucleotide LY2181308 in combination with 
docetaxel for second-line treatment of patients with non-small-cell lung cancer: a randomized open-label phase II study. J. Thorac. Oncol. 9, 17048 (2014).

59. Wiechno, P. et al. A randomised phase 2 study combining LY2181308 sodium (survivin antisense oligonucleotide) with first-line docetaxel/prednisone in patients with castration-resistant prostate cancer. Eur. Urol. 65, 516-20 (2014).

60. Erba, H. P. et al. Safety and Pharmacokinetics of the Antisense Oligonucleotide (ASO) LY2181308 as a Single-Agent or in Combination with Idarubicin and Cytarabine in Patients with Refractory or Relapsed Acute Myeloid Leukemia (AML). Invest. New Drugs 31, 1023-1034 (2014).

61. Tolcher, A. W. et al. Phase I and pharmacokinetic study of YM155, a smallmolecule inhibitor of survivin. J. Clin. Oncol. 26, 5198-203 (2008).

62. Satoh, T. et al. Phase I study of YM155, a novel survivin suppressant, in patients with advanced solid tumors. Clin. cancer Res. 15, 3872-80 (2009).

63. Tolcher, a W. et al. A phase II study of YM155, a novel small-molecule suppressor of survivin, in castration-resistant taxane-pretreated prostate cancer. Ann. Oncol. 23, 968-73 (2012). 
64. Cheson, B. D. et al. A phase II study of the survivin suppressant YM155 in patients with refractory diffuse large B-cell lymphoma. Cancer 118, 3128-34 (2012).

65. Lewis, K. D. et al. A multi-center phase II evaluation of the small molecule survivin suppressor YM155 in patients with unresectable stage III or IV melanoma. Invest. New Drugs 29, 161-6 (2011).

66. Hassan, R. et al. Detection and quantitation of serum mesothelin, a tumor marker for patients with mesothelioma and ovarian cancer. Clin. Cancer Res. 12, 447-53 (2006).

67. Shariat, S. F. et al. Urine detection of survivin is a sensitive marker for the noninvasive diagnosis of bladder cancer. J. Urol. 171, 626-30 (2004).

68. Das, A., Tan, W.-L., Teo, J. \& Smith, D. R. Expression of survivin in primary glioblastomas. J. Cancer Res. Clin. Oncol. 128, 302-6 (2002).

69. Pennati, M. et al. Radiosensitization of human melanoma cells by ribozymemediated inhibition of survivin expression. J. Invest. Dermatol. 120, 648-54 (2003).

70. Moriai, R., Tsuji, N., Moriai, M., Kobayashi, D. \& Watanabe, N. Survivin plays as a resistant factor against tamoxifen-induced apoptosis in human breast cancer cells. Breast Cancer Res. Treat. 117, 261-71 (2009). 
71. McGrogan, B. T., Gilmartin, B., Carney, D. N. \& McCann, A. Taxanes, microtubules and chemoresistant breast cancer. Biochim. Biophys. Acta 1785, 96-132 (2008).

72. Liu, W.-S. et al. siRNA directed against survivin enhances pancreatic cancer cell gemcitabine chemosensitivity. Dig. Dis. Sci. 54, 89-96 (2009).

73. Lu, C., Altieri, D. C. \& Tanigawa, N. Expression of A Novel Antiapoptosis Gene , Survivin , Correlated with Tumor Cell Apoptosis and p53 Accumulation in Gastric Carcinomas Apoptosis and p53 Accumulation in Gastric Carcinomas. Cancer Res. 58, 1808-1812 (1998).

74. Chakravarti, B. A. et al. Quantitatively determined survivin expression levels are of Prognostic Value in Human Gliomas. J. Clin. Oncol. 20, 1063-1068 (2002).

75. Kappler, M. et al. Knockdown of survivin expression by small interfering RNA reduces the clonogenic survival of human sarcoma cell lines independently of p53. Cancer Gene Ther. 11, 186-93 (2004).

76. Li, H., Niederkorn, J. Y., Neelam, S. \& Alizadeh, H. Downregulation of survivin expression enhances sensitivity of cultured uveal melanoma cells to cisplatin treatment. Exp. Eye Res. 83, 176-82 (2006).

77. Becker, J. C. et al. Survivin-specific T-cell reactivity correlates with tumor response and patient survival: a phase-II peptide vaccination trial in 
metastatic melanoma. Cancer Immunol. Immunother. (2012). doi:10.1007/s00262-012-1266-9

78. Andersen, M. H., Becker, J. C. \& Straten, P. T. Regulators of apoptosis: suitable targets for immune therapy of cancer. Nat. Rev. Drug Discov. 4, 399-409 (2005).

79. Otto, K. et al. Lack of toxicity of therapy-induced T cell responses against the universal tumour antigen survivin. Vaccine 23, 884-9 (2005).

80. Schmidt, S. M. et al. Survivin is a shared tumor-associated antigen expressed in a broad variety of malignancies and recognized by specific cytotoxic T cells. Immunobiology 102, 571-576 (2003).

81. Xiang, R. et al. A DNA vaccine targeting survivin combines apoptosis with suppression of angiogenesis in lung tumor eradication. Cancer Res. 65, 553-61 (2005).

82. Arber, C. et al. Survivin-specific T cell receptor targets tumor but not T cells. J. Clin. Invest. 125, 157-168 (2015).

83. Altieri, D. C. Validating survivin as a cancer therapeutic target. Nat. Rev. Cancer 3, 46-54 (2003).

84. Li, F. Survivin study: what is the next wave? J. Cell. Physiol. 197, 8-29 (2003). 
85. Altieri, D. C. The molecular basis and potential role of survivin in cancer diagnosis and therapy. Trends Mol. Med. 7, 542-7 (2001).

86. Chen, X., Duan, N., Zhang, C. \& Zhang, W. Survivin and Tumorigenesis: Molecular Mechanisms and Therapeutic Strategies. J. Cancer 7, 314-23 (2016).

87. Shin, S. et al. An anti-apoptotic protein human survivin is a direct inhibitor of caspase-3 and -7. Biochemistry 40, 1117-23 (2001).

88. Zaffaroni, N. \& Daidone, M. G. Survivin expression and resistance to anticancer treatments: perspectives for new therapeutic interventions. Drug Resist. Updat. 5, 65-72 (2002).

89. Beardmore, V. a, Ahonen, L. J., Gorbsky, G. J. \& Kallio, M. J. Survivin dynamics increases at centromeres during G2/M phase transition and is regulated by microtubule-attachment and Aurora B kinase activity. J. Cell Sci. 117, 4033-42 (2004).

90. Uren, A. G. et al. Survivin and the inner centromere protein INCENP show similar cell-cycle localization and gene knockout phenotype. Curr. Biol. 10, 1319-1328 (2000).

91. Carrasco, R. a et al. Antisense inhibition of survivin expression as a cancer therapeutic. Mol. Cancer Ther. 10, 221-32 (2011). 
92. Jeyaprakash, a A. et al. Structure of a Survivin-Borealin-INCENP core complex reveals how chromosomal passengers travel together. Cell 131, $271-85$ (2007).

93. Li, F. et al. Control of apoptosis and mitotic spindle checkpoint by survivin. Nature 396, 580-584 (1998).

94. Hoffman, W. H., Biade, S., Zilfou, J. T., Chen, J. \& Murphy, M. Transcriptional repression of the anti-apoptotic survivin gene by wild type p53. J. Biol. Chem. 277, 3247-57 (2002).

95. Jin, Q., Menter, D. G., Mao, L., Hong, W. K. \& Lee, H.-Y. Survivin expression in normal human bronchial epithelial cells: an early and critical step in tumorigenesis induced by tobacco exposure. Carcinogenesis 29, 1614-22 (2008).

96. Tamm, I. et al. IAP-Family Protein Survivin Inhibits Caspase Activity and Apoptosis Induced by Fas (CD95), Bax, Caspases, and Anticancer Drugs. Cancer Res. 58, 5315-5320 (1998).

97. Tanioka, M. et al. Phase I study of LY2181308, an antisense oligonucleotide against survivin, in patients with advanced solid tumors. Cancer Chemother. Pharmacol. 68, 505-11 (2011).

98. Carter, B. Z., Wang, R., Schober, W. D., Milella, M. \& Andreeff, M. Targeting Survivin Expression Induces Cell Proliferation Defect and Subsequent Cell 
Death Involving Mitochondrial Pathway in Myeloid Leukemic Cell. Cell Cycle 2, 488-493 (2003).

99. Ansell, S. M. et al. Inhibition of survivin expression suppresses the growth of aggressive non-Hodgkin's lymphoma. Leukemia 18, 616-23 (2004).

100. Nakahara, T. et al. YM155, a novel small-molecule survivin suppressant, induces regression of established human hormone-refractory prostate tumor xenografts. Cancer Res. 67, 8014-21 (2007).

101. Nakahara, T. et al. Broad spectrum and potent antitumor activities of YM155, a novel small-molecule survivin suppressant, in a wide variety of human cancer cell lines and xenograft models. Cancer Sci. 102, 614-21 (2011).

102. Iwasa, T. et al. Radiosensitizing effect of YM155, a novel small-molecule survivin suppressant, in non-small cell lung cancer cell lines. Clin. cancer Res. 14, 6496-504 (2008).

103. Andersen, M. H., Pedersen, L. Ø. \& Becker, J. C. Identification of a Cytotoxic T Lymphocyte Response to the Apoptosis Inhibitor Protein Survivin in Cancer Patients. Cancer Res. 61, 869-872 (2000).

104. Bachinsky, M. M. et al. Mapping and binding analysis of peptides derived from the tumor-associated antigen survivin for eight HLA alleles. Cancer Immun. 5, 6-15 (2005). 
105. Andersen, M. H. et al. Spontaneous Cytotoxic T-Cell Responses against Survivin-derived MHC Class I-restricted T-Cell Epitopes in Situ As Well As ex Vivo in Cancer Patients Advances in Brief As Well As ex Vivo in Cancer Patients 1. Cancer Res. 61, 5964-5968 (2001).

106. Bera, T. K. \& Pastan, I. Mesothelin Is Not Required for Normal Mouse Development or Reproduction. Mol. Cell. Biol. 20, 2902-2906 (2000).

107. Cheng, W.-F. et al. High mesothelin correlates with chemoresistance and poor survival in epithelial ovarian carcinoma. Br. J. Cancer 100, 1144-53 (2009).

108. Alvarez, H. et al. Mesothelin is a specific biomarker of invasive cancer in the Barrett-associated adenocarcinoma progression model: translational implications for diagnosis and therapy. Nanomedicine 4, 295-301 (2008).

109. Chang, M.-C. et al. Mesothelin enhances invasion of ovarian cancer by inducing MMP-7 through MAPK/ERK and JNK pathways. Biochem. J. 442, 293-302 (2012).

110. Smolková, P. et al. Validity of Mesothelin in Occupational Medicine Practice. Int. J. Occup. Med. Environ. Health 29, (2016).

111. Kipps, E., Tan, D. S. P. \& Kaye, S. B. Meeting the challenge of ascites in ovarian cancer: new avenues for therapy and research. Nat. Rev. Cancer 13, 273-82 (2013). 
112. Bharadwaj, U., Marin-Muller, C., Li, M., Chen, C. \& Yao, Q. Mesothelin overexpression promotes autocrine IL-6/sIL-6R trans-signaling to stimulate pancreatic cancer cell proliferation. Carcinogenesis 32, 1013-24 (2011).

113. Hwang, H. C. \& Clurman, B. E. Cyclin E in normal and neoplastic cell cycles. Oncogene 24, 2776-86 (2005).

114. Bharadwaj, U., Li, M., Chen, C. \& Yao, Q. Mesothelin-induced pancreatic cancer cell proliferation involves alteration of cyclin E via activation of signal transducer and activator of transcription protein 3. Mol. Cancer Res. 6, 1755-65 (2008).

115. Zhang, D. et al. Deficiency of the Erc/mesothelin gene ameliorates renal carcinogenesis in Tsc2 knockout mice. Cancer Sci. 102, 720-7 (2011).

116. Ito, T. et al. ERC/mesothelin is expressed in human gastric cancer tissues and cell lines. Oncol. Rep. 31, 27-33 (2014).

117. Rump, A. et al. Binding of ovarian cancer antigen CA125/MUC16 to mesothelin mediates cell adhesion. J. Biol. Chem. 279, 9190-8 (2004).

118. Melaiu, O. et al. A review of transcriptome studies combined with data mining reveals novel potential markers of malignant pleural mesothelioma. Mutat. Res. 750, 132-40 (2012).

119. Ordóñez, N. G. Application of Mesothelin Immunostaining in Tumor Diagnosis. Am. J. Surg. Pathol. 27, 1418-1428 (2003). 
120. Drapkin, R., Crum, C. P. \& Hecht, J. L. Expression of candidate tumor markers in ovarian carcinoma and benign ovary: Evidence for a link between epithelial phenotype and neoplasia. Hum. Pathol. 35, 1014-1021 (2004).

121. Ordóñez, N. G. \& Sahin, A. a. Diagnostic utility of immunohistochemistry in distinguishing between epithelioid pleural mesotheliomas and breast carcinomas: a comparative study. Hum. Pathol. 45, 1529-40 (2014).

122. Erdogan, E. et al. Incidental localized (solitary) mediastinal malignant mesothelioma. Br. J. Radiol. 78, 858-61 (2005).

123. Shimizu, A. et al. Coexpression of MUC16 and mesothelin is related to the invasion process in pancreatic ductal adenocarcinoma. Cancer Sci. 103, 739-46 (2012).

124. Hassan, R. \& Ho, M. Mesothelin targeted cancer immunotherapy. Eur. J. Cancer 44, 46-53 (2008).

125. Panou, V. et al. The established and future biomarkers of malignant pleural mesothelioma. Cancer Treat. Rev. 41, 486-495 (2015).

126. Tajima, K. et al. ERC/Mesothelin as a Marker for Chemotherapeutic Response in Patients with Mesothelioma. Anticancer Res. 28, 3933-3936 (2008).

127. Wang, K. et al. Inhibition of mesothelin as a novel strategy for targeting cancer cells. PLoS One 7, e33214 (2012). 
128. Sage, E. et al. Mesothelin Is Overexpressed in the Vast Majority of Ductal Adenocarcinomas of the Pancreas: Identification of a New Pancreatic Cancer Marker by Serial Analysis of Gene. Clin. cancer Res. 7, 3862-3868 (2001).

129. Creaney, J., Dick, I. M. \& Robinson, B. W. S. Discovery of new biomarkers for malignant mesothelioma. Curr. Pulmonol. reports 4, 15-21 (2015).

130. Hollevoet, K. et al. Serum Mesothelin for Diagnosing Malignant Pleural Mesothelioma: An Individual Patient Data Meta-Analysis. J. Clin. Oncol. 30, 1541-1549 (2012).

131. Hassan, R. et al. Phase I study of SS1P, a recombinant anti-mesothelin immunotoxin given as a bolus I.V. infusion to patients with mesothelinexpressing mesothelioma, ovarian, and pancreatic cancers. Clin. Cancer Res. 13, 5144-9 (2007).

132. Hassan, R. et al. Antitumor Activity of SS (dsFv) PE38 and SS1 (dsFv) PE38, Recombinant Antimesothelin Immunotoxins against Human Gynecologic Cancers Grown in Organotypic Culture in Vitro 1. Clin. Cancer Res. 8, 35203526 (2002).

133. Li, Q., Verschraegen, C. F., Mendoza, J. \& Hassan, R. Cytotoxic Activity of the Recombinant Anti-mesothelin Immunotoxin, SS1 (dsFv) PE38, Towards Tumor Cell Lines Established from Ascites of Patients with Peritoneal Mesotheliomas. Anticancer Agents Med Chem 24, 1327-1335 (2004). 
134. Filpula, D. et al. Releasable PEGylation of mesothelin targeted immunotoxin SS1P achieves single dosage complete regression of a human carcinoma in mice. Bioconjug. Chem. 18, 773-84 (2007).

135. Hassan, R. et al. Tumor-directed radiation and the immunotoxin SS1P in the treatment of mesothelin-expressing tumor xenografts. Clin. cancer Res. 12, 4983-8 (2006).

136. Zhang, Y. et al. Synergistic antitumor activity of taxol and immunotoxin SS1P in tumor-bearing mice. Clin. cancer Res. 12, 4695-701 (2006).

137. Hassan, R. et al. Major cancer regressions in mesothelioma after treatment with an anti-mesothelin immunotoxin and immune suppression. Sci. Transl. Med. 5, 208ra147 (2013).

138. Thomas, A. M. et al. Mesothelin-specific CD8(+) T cell responses provide evidence of in vivo cross-priming by antigen-presenting cells in vaccinated pancreatic cancer patients. J. Exp. Med. 200, 297-306 (2004).

139. Hassan, R. et al. Phase I clinical trial of the chimeric anti-mesothelin monoclonal antibody MORAb-009 in patients with mesothelin-expressing cancers. Clin. cancer Res. 16, 6132-8 (2010).

140. Hassan, R. et al. Preclinical evaluation of MORAb-009, a chimeric antibody targeting tumor-associated mesothelin. Cancer Immun. 7, 20-30 (2007). 
141. Holsapple, M. P. et al. Research strategies for safety evaluation of nanomaterials, part II: toxicological and safety evaluation of nanomaterials, current challenges and data needs. Toxicol. Sci. 88, 12-7 (2005).

142. Love, S. a, Maurer-Jones, M. a, Thompson, J. W., Lin, Y.-S. \& Haynes, C. L. Assessing nanoparticle toxicity. Annu. Rev. Anal. Chem. (Palo Alto. Calif). 5, 181-205 (2012).

143. Lewinski, N., Colvin, V. \& Drezek, R. Cytotoxicity of nanoparticles. Small 4, $26-49$ (2008).

144. Kroll, A., Pillukat, M. H., Hahn, D. \& Schnekenburger, J. Current in vitro methods in nanoparticle risk assessment: limitations and challenges. Eur. J. Pharm. Biopharm. 72, 370-7 (2009).

145. Conde, J. J. et al. Revisiting 30 years of biofunctionalization and surface chemistry of inorganic nanoparticles for nanomedicine. Front. Chem. 2, 127 (2014).

146. Warheit, D. B. How Meaningful are the Results of Nanotoxicity Studies in the Absence of Adequate Material Characterization? Toxicol. Sci. 101, 183-185 (2008).

147. De Volder, M. F. L., Tawfick, S. H., Baughman, R. H. \& Hart, a J. Carbon nanotubes: present and future commercial applications. Science (80-. ). 339, 535-9 (2013). 
148. Burtea, C. et al. In vitro biomedical applications of functionalized iron oxide nanoparticles, including those not related to magnetic properties. Contrast Media Mol. Imaging 6, 236-50 (2011).

149. Lu, M., Cohen, M. H., Rieves, D. \& Pazdur, R. FDA report: Ferumoxytol for intravenous iron therapy in adult patients with chronic kidney disease. Am. J. Hematol. 85, 315-319 (2010).

150. Jin, R., Lin, B., Li, D. \& Ai, H. Superparamagnetic iron oxide nanoparticles for MR imaging and therapy: design considerations and clinical applications. Curr. Opin. Pharmacol. 18, 18-27 (2014).

151. Reddy, L. H., Arias, J. L., Nicolas, J. \& Couvreur, P. Magnetic nanoparticles: design and characterization, toxicity and biocompatibility, pharmaceutical and biomedical applications. Chem. Rev. 112, 5818-78 (2012).

152. Wahajuddin \& Arora, S. Superparamagnetic iron oxide nanoparticles: magnetic nanoplatforms as drug carriers. Int. J. Nanomedicine 7, 3445-71 (2012).

153. Alexis, F., Pridgen, E., Molnar, L. K. \& Farokhzad, O. C. Factors Affecting the Clearance and Biodistribution of Polymeric Nanoparticles. Mol. Pharm. 5, 505-515 (2008).

154. Mornet, S., Vasseur, S., Grasset, F. \& Duguet, E. Magnetic nanoparticle design for medical diagnosis and therapy. J. Mater. Chem. 14, 2161 (2004). 
155. Marquis, B. J., Love, S. a, Braun, K. L. \& Haynes, C. L. Analytical methods to assess nanoparticle toxicity. Analyst 134, 425-39 (2009).

156. Monteiro-Riviere, N. a, Inman, a O. \& Zhang, L. W. Limitations and relative utility of screening assays to assess engineered nanoparticle toxicity in a human cell line. Toxicol. Appl. Pharmacol. 234, 222-35 (2009).

157. Mahmoudi, M., Laurent, S., Shokrgozar, M. a \& Hosseinkhani, M. Toxicity evaluations of superparamagnetic iron oxide nanoparticles: cell "vision" versus physicochemical properties of nanoparticles. ACS Nano 5, 7263-76 (2011).

158. Mikhaylova, M. et al. Superparamagnetism of magnetite nanoparticles: dependence on surface modification. Langmuir 20, 2472-7 (2004).

159. Yu, W. W., Chang, E., Sayes, C. M., Drezek, R. \& Colvin, V. L. Aqueous dispersion of monodisperse magnetic iron oxide nanocrystals through phase transfer. Nanotechnology 17, 4483-4487 (2006).

160. Tassa, C., Shaw, S. Y. \& Weissleder, R. Dextran-Coated Iron Oxide Nanoparticles: a Versatile Platform for Targeted Molecular Imaging, Molecular Diagnostics and Therapy. Acc Chem Res 44, 842-852 (2011).

161. Muldoon, L. L., S??ndor, M., Pinkston, K. E. \& Neuwelt, E. a. Imaging, Distribution, and Toxicity of Superparamagnetic Iron Oxide Magnetic 
Resonance Nanoparticles in the Rat Brain and Intracerebral Tumor. Neurosurgery 57, 785-796 (2005).

162. Soenen, S. J., De Cuyper, M., De Smedt, S. C. \& Braeckmans, K. Investigating the toxic effects of iron oxide nanoparticles. Methods Enzymol. 509, 195-224 (Elsevier Inc., 2012).

163. Wo, J. M., Pulskamp, K. \& Krug, H. F. Oops They Did It Again! Carbon Nanotubes Hoax Scientists in Viability Assays. Nano Lett. 6, 1261-1268 (2006).

164. Liu, Y., Zhao, Y., Sun, B. \& Chen, C. Understanding the Toxicity of Carbon Nanotubes. Acc. Chem. Res. 46, 702-713 (2013).

165. Gangwal, S. et al. Informing Selection of Nanomaterial Concentrations for ToxCast in Vitro Testing Based on Occupational Exposure Potential. Environ. Health Perspect. 119, 1539-1547 (2011).

166. Oberdörster, G. Nanotoxicology: in vitro - in vivo Dosimetry. Environ. Health Perspect. 120, 13- (2012).

167. Shi, J., Xiao, Z., Kamaly, N. \& Farokhzad, O. C. Self-assembled targeted nanoparticles: evolution of technologies and bench to bedside translation. Acc. Chem. Res. 44, 1123-34 (2011). 
168. Heyder, J. Deposition of inhaled particles in the human respiratory tract and consequences for regional targeting in respiratory drug delivery. Proc. Am. Thorac. Soc. 1, 315-20 (2004).

169. Schweiger, C. et al. Quantification of the internalization patterns of superparamagnetic iron oxide nanoparticles with opposite charge. J. Nanobiotechnology 10, 28 (2012).

170. Chen, D. et al. Gene expression profile of human lung epithelial cells chronically exposed to single-walled carbon nanotubes. Nanoscale Res. Lett. 10, (2015).

171. Thurnherr, T. et al. A comparison of acute and long-term effects of industrial multiwalled carbon nanotubes on human lung and immune cells in vitro. Toxicol. Lett. 200, 176-86 (2011).

172. Wang, L. et al. Carbon Nanotubes Induce Malignant Transformation and Tumorigenesis of Human Lung Epithelial Cells. Nano Lett. 11, 2796-2803 (2011).

173. Luanpitpong, S., Wang, L., Castranova, V. \& Rojanasakul, Y. Induction of stem-like cells with malignant properties by chronic exposure of human lung epithelial cells to single-walled carbon nanotubes. Part. Fibre Toxicol. 11, 22 (2014). 
174. Luanpitpong, S., Wang, L., Davidson, D. C., Riedel, H. \& Rojanasakul, Y. Carcinogenic Potential for High Aspect Ratio Carbon Nanomaterials. Environ. Sci. Nano 1-20 (2016).

175. Wang, L. et al. Neoplastic-like transformation effect of single-walled and multi-walled carbon nanotubes compared to asbestos on human lung small airway epithelial cells. Nanotoxicology 8, 485-507 (2014).

176. Medina, C., Santos-Martinez, M. J., Radomski, a, Corrigan, O. I. \& Radomski, M. W. Nanoparticles: pharmacological and toxicological significance. Br. J. Pharmacol. 150, 552-8 (2007).

177. Schnorr, J. M. \& Swager, T. M. Emerging Applications of Carbon Nanotubes. Chem. Mater. 23, 646-657 (2011).

178. Delogu, L. G. et al. Functionalized multiwalled carbon nanotubes as ultrasound contrast agents. Proc. Natl. Acad. Sci. U. S. A. 109, 16612-7 (2012).

179. Petri-Fink, A., Steitz, B., Finka, A., Salaklang, J. \& Hofmann, H. Effect of cell media on polymer coated superparamagnetic iron oxide nanoparticles (SPIONs): colloidal stability, cytotoxicity, and cellular uptake studies. Eur. J. Pharm. Biopharm. 68, 129-37 (2008). 
180. Mahmoudi, B. M., Simchi, A., Vali, H. \& Imani, M. Cytotoxicity and Cell Cycle Effects of Bare and Poly (vinyl alcohol)-Coated Iron Oxide Nanoparticles in Mouse Fibroblasts. Adv. Biomater. 11, 243-250 (2009).

181. Wada, S. et al. New local hyperthermia using dextran magnetite complex (DM) for oral cavity: experimental study in normal hamster tongue. Oral Dis. 7, 192-195 (2001).

182. Hu, F., Neoh, K. G., Cen, L. \& Kang, E.-T. Cellular response to magnetic nanoparticles "PEGylated" via surface-initiated atom transfer radical polymerization. Biomacromolecules 7, 809-16 (2006).

183. Wu, P. et al. Biocompatible Carbon Nanotubes Generated by Functionalization with Glycodendrimers. Angew. Chem. Int. Ed. Engl. 47, 5022-5025 (2010).

184. Wagner, K. et al. Synthesis of oligonucleotide-functionalized magnetic nanoparticles and study on their in vitro cell uptake. Appl. Organomet. Chem. 18, 514-519 (2004).

185. Wang, A. Z. et al. Superparamagnetic iron oxide nanoparticle-aptamer bioconjugates for combined prostate cancer imaging and therapy. ChemMedChem 3, 1311-5 (2008).

186. Mahajan, S., Koul, V., Choudhary, V., Shishodia, G. \& Bharti, A. C. Preparation and in vitro evaluation of folate-receptor-targeted SPION- 
polymer micelle hybrids for MRI contrast enhancement in cancer imaging. Nanotechnology 24, 015603 (2013).

187. Fan, C. et al. Tumor selectivity of stealth multi-functionalized superparamagnetic iron oxide nanoparticles. Int. J. Pharm. 404, 180-90 (2011).

188. Kim, S. H., Jeong, J. H., Chun, K. W. \& Park, T. G. Target-specific cellular uptake of PLGA nanoparticles coated with poly(L-lysine)-poly(ethylene glycol)-folate conjugate. Langmuir 21, 8852-7 (2005).

189. Dey, S. \& Maiti, T. K. Superparamagnetic Nanoparticles and RNAi-Mediated Gene Silencing: Evolving Class of Cancer Diagnostics and Therapeutics. J. Nanomater. 2012, 1-15 (2012).

190. Hoebeke, J. et al. Design of Folic Acid-Conjugated Nanoparticles for Drug Targeting. J. Pharm. Sci. 89, 1452-1464 (2000).

191. Schulze, C. et al. Not ready to use - overcoming pitfalls when dispersing nanoparticles in physiological media. Nanotoxicology 2, 51-61 (2009).

192. Laurent, S. \& Mahmoudi, M. Superparamagnetic iron oxide nanoparticles: promises for diagnosis and treatment of cancer. Int. J. Mol. Epidemiol. Genet. 2, 367-90 (2011).

193. Ai, J. et al. Nanotoxicology and nanoparticle safety in biomedical designs. Int. J. Nanomedicine 6, 1117-27 (2011). 
194. Li, S.-D. \& Huang, L. Targeted delivery of antisense oligodeoxynucleotide and small interference RNA into lung cancer cells. Mol. Pharm. 3, 579-88 (2006).

195. Kaaki, K. et al. Magnetic nanocarriers of doxorubicin coated with poly(ethylene glycol) and folic acid: relation between coating structure, surface properties, colloidal stability, and cancer cell targeting. Langmuir 28, 1496-505 (2012).

196. Omidirad, R., Rajabi Hosseinpour, F. \& Farahani, B. Preparation and in vitro drug delivery response of doxorubicin loaded PAA coated magnetite nanoparticles. J. Serbian Chem. Soc. 78, 1609-1616 (2013).

197. Neuberger, T., Schöpf, B., Hofmann, H., Hofmann, M. \& von Rechenberg, B. Superparamagnetic nanoparticles for biomedical applications: Possibilities and limitations of a new drug delivery system. J. Magn. Magn. Mater. 293, 483-496 (2005).

198. Safi, M., Sarrouj, H., Sandre, O., Mignet, N. \& Berret, J.-F. Interactions between sub-10-nm iron and cerium oxide nanoparticles and 3T3 fibroblasts: the role of the coating and aggregation state. Nanotechnology 21, 145103 (2010).

199. Jain, T. K., Reddy, M. K., Morales, M. A., Leslie-pelecky, D. L. \& Labhasetwar, V. Biodistribution, Clearance, and Biocompatibility of Iron Oxide Magnetic Nanoparticles in Rats. Mol. Pharm. 5, 316-327 (2008). 
200. Mahmoudi, M., Sant, S., Wang, B., Laurent, S. \& Sen, T. Superparamagnetic iron oxide nanoparticles (SPIONs): development, surface modification and applications in chemotherapy. Adv. Drug Deliv. Rev. 63, 24-46 (2011).

201. Hrkach, J. et al. Preclinical development and clinical translation of a PSMAtargeted docetaxel nanoparticle with a differentiated pharmacological profile. Sci. Transl. Med. 4, 128ra39 (2012).

202. Zhang, K., Hao, L., Hurst, S. J. \& Mirkin, C. a. Antibody-linked spherical nucleic acids for cellular targeting. J. Am. Chem. Soc. 134, 16488-91 (2012).

203. Wan, S. et al. Biocompatible superparamagnetic iron oxide nanoparticle dispersions stabilized with poly ( ethylene glycol ) - oligo ( aspartic acid) hybrids. J. Biomed. Mater. Res. Part A 946-954 (2006). doi:10.1002/jbm.a

204. Hyeon, T., Lee, S. S., Park, J., Chung, Y. \& Na, H. B. Synthesis of highly crystalline and monodisperse maghemite nanocrystallites without a sizeselection process. J. Am. Chem. Soc. 123, 12798-801 (2001).

205. Smith, A. M. \& Nie, S. Minimizing the hydrodynamic size of quantum dots with multifunctional multidentate polymer ligands. J. Am. Chem. Soc. 130, $11278-9$ (2008).

206. Shen, H., Jawaid, A. M. \& Snee, P. T. Poly(ethylene glycol) carbodiimide coupling reagents for the biological and chemical functionalization of watersoluble nanoparticles. ACS Nano 3, 915-23 (2009). 
207. Sato, K., Tachibana, Y., Hattori, S., Chiba, T. \& Kuwabata, S. Polyacrylic acid coating of highly luminescent CdS nanocrystals for biological labeling applications. J. Colloid Interface Sci. 324, 257-60 (2008).

208. Shen, H., Jawaid, A. M. \& Snee, P. T. Poly (ethylene glycol) Carbodiimide Coupling Reagents for the Biological and Chemical Functionalization of Water- Soluble Nanoparticles. ACS Nano 3, 915-23 (2009).

209. Carmen Bautista, M., Bomati-Miguel, O., del Puerto Morales, M., Serna, C. J. \& Veintemillas-Verdaguer, S. Surface characterisation of dextran-coated iron oxide nanoparticles prepared by laser pyrolysis and coprecipitation. J. Magn. Magn. Mater. 293, 20-27 (2005).

210. Thorek, D. L. J., Elias, D. R. \& Tsourkas, A. Comparative Analysis of Nanoparticle-Antibody Conjugations: Carbodiimide versus Click Chemistry. Mol. Imaging 8, 221-229 (2009).

211. Gupta, A. K. \& Gupta, M. Cytotoxicity suppression and cellular uptake enhancement of surface modified magnetic nanoparticles. Biomaterials 26, 1565-73 (2005).

212. Massich, M. D., Giljohann, D. A., Schmucker, A. L., Patel, P. C. \& Mirkin, C. A. Cellular Response of Polyvalent Oligonucleotide - Gold Nanoparticle Conjugates. ACS Nano 4, 5641-5646 (2010). 
213. Rosi, N. L. et al. Oligonucleotide-modified gold nanoparticles for intracellular gene regulation. Science (80-. ). 312, 1027-30 (2006).

214. Yu, M. K. et al. Drug-loaded superparamagnetic iron oxide nanoparticles for combined cancer imaging and therapy in vivo. Angew. Chem. Int. Ed. Engl. 47, 5362-5 (2008).

215. Santra, S., Kaittanis, C., Grimm, J. \& Perez, J. M. Drug/dye-Loaded, Multifunctional Iron Oxide Nanoparticles for Combined Targeted Cancer Therapy and Dual Optical/MR-Imaging. Small 5, 1862-8 (2009).

216. Thompson, K. \& Michielsen, S. Novel synthesis of N-substituted polyacrylamides: Derivatization of poly(acrylic acid) with amines using a triazine-based condensing reagent. J. Polym. Sci. Part A Polym. Chem. 44, $126-136$ (2006).

217. Wrobel, N., Schinkinger, M. \& Mirsky, V. M. A novel ultraviolet assay for testing side reactions of carbodiimides. Anal. Biochem. 305, 135-8 (2002).

218. Palazon, F. et al. Carbodiimide/NHS Derivatization of $\mathrm{COOH}-$ Terminated SAMs: Activation or Byproduct Formation? Langmuir 30, 4545-4550 (2014).

219. Kocbek, P., Obermajer, N., Cegnar, M., Kos, J. \& Kristl, J. Targeting cancer cells using PLGA nanoparticles surface modified with monoclonal antibody. J. Control. release 120, 18-26 (2007). 
220. Natarajan, A. et al. NanoFerrite Particle Based Radioimmunonanoparticles: Binding affinity and in vivo pharmacokinetics. Bioconjug. Chem. 19, 12111218 (2008).

221. Sam, S. et al. Semiquantitative study of the EDC/NHS activation of acid terminal groups at modified porous silicon surfaces. Langmuir 26, 809-14 (2010).

222. Wang, T.-H. \& Lee, W.-C. Immobilization of proteins on magnetic nanoparticles. Biotechnol. Bioprocess Eng. 8, 263-267 (2003).

223. Walsh, M. K., Wang, X. \& Weimer, B. C. Optimizing the immobilization of single-stranded DNA onto glass beads. J. Biochem. Biophys. Methods 47, 221-231 (2001).

224. Christiaens, P. et al. EDC-mediated DNA attachment to nanocrystalline CVD diamond films. Biosens. Bioelectron. 22, 170-177 (2006).

225. Millan, K. M., Saraullo, a \& Mikkelsen, S. R. Voltammetric DNA biosensor for cystic fibrosis based on a modified carbon paste electrode. Anal. Chem. 66, 2943-2948 (1994).

226. Booth, M. A., Kannappan, K., Hosseini, A. \& Partridge, A. In-Depth Electrochemical Investigation of Surface Attachment Chemistry via Carbodiimide Coupling. Langmuir 31, 8033-41 (2015). 
227. Rosen, J. E., Chan, L., Shieh, D.-B. \& Gu, F. X. Iron oxide nanoparticles for targeted cancer imaging and diagnostics. Nanomedicine 8, 275-90 (2012).

228. Huber, D. L. Synthesis, properties, and applications of iron nanoparticles. Small 1, 482-501 (2005).

229. Mccormack, P. L. Ferumoxytol In Iron Deficiency Anaemia in Adults with Chronic Kidney Disease. Drugs 72, 2013-2022 (2012).

230. Zhu, L. et al. Multifunctional pH-sensitive superparamagnetic iron-oxide nanocomposites for targeted drug delivery and MR imaging. J. Control. Release 169, 228-38 (2013).

231. Akbarzadeh, A. et al. Synthesis, characterization, and in vitro evaluation of novel polymer-coated magnetic nanoparticles for controlled delivery of doxorubicin. Nanotechnol. Sci. Appl. 5, 13-25 (2012).

232. Wilson, M. W. et al. Hepatocellular carcinoma: regional therapy with a magnetic targeted carrier bound to doxorubicin in a dual MR imaging/ conventional angiography suite--initial experience with four patients. Radiology 230, 287-93 (2004).

233. Munnier, E. et al. Novel method of doxorubicin-SPION reversible association for magnetic drug targeting. Int. J. Pharm. 363, 170-6 (2008).

234. Dósa, E. et al. MRI using ferumoxytol improves the visualization of central nervous system vascular malformations. Stroke 42, 1581-8 (2011). 
235. Okuhata, Y. Delivery of diagnostic agents for magnetic resonance imaging. Adv. Drug Deliv. Rev. 37, 121-137 (1999).

236. Weinmann, H.-J., Ebert, W., Misselwitz, B. \& Schmitt-Willich, H. Tissuespecific MR contrast agents. Eur. J. Radiol. 46, 33-44 (2003).

237. Lee, G. Y. et al. Theranostic Nanoparticles with Controlled Release of Gemcitabine for Targeted Therapy and MRI of Pancreatic Cancer. ACS Nano 7, 2078-2089 (2013).

238. Kumar, M., Yigit, M., Dai, G., Moore, A. \& Medarova, Z. Image-guided breast tumor therapy using a small interfering RNA nanodrug. Cancer Res. 70, 7553-7561 (2010).

239. Geinguenaud, F., Souissi, I., Fagard, R., Motte, L. \& Lalatonne, Y. Electrostatic assembly of a DNA superparamagnetic nano-tool for simultaneous intracellular delivery and in situ monitoring. Nanomedicine $\mathbf{8}$, $1106-15$ (2012).

240. Cutler, J. I., Zheng, D., Xu, X., Giljohann, D. a \& Mirkin, C. a. Polyvalent oligonucleotide iron oxide nanoparticle "click" conjugates. Nano Lett. 10, 1477-80 (2010).

241. Giljohann, D. a et al. Oligonucleotide loading determines cellular uptake of DNA-modified gold nanoparticles. Nano Lett. 7, 3818-21 (2007). 
242. Jensen, S. a et al. Spherical nucleic acid nanoparticle conjugates as an RNAi-based therapy for glioblastoma. Sci. Transl. Med. 5, 209ra152 (2013).

243. Feazell, R. P., Nakayama-Ratchford, N., Dai, H. \& Lippard, S. J. Soluble single-walled carbon nanotubes as longboat delivery systems for platinum(IV) anticancer drug design. J. Am. Chem. Soc. 129, 8438-9 (2007).

244. Pastorin, G. et al. Double functionalisation of carbon nanotubes for multimodal drug delivery. Chem. Commun. 1, 1182-4 (2006).

245. Singh, R. et al. Binding and Condensation of Plasmid DNA onto Functionalized Carbon Nanotubes: Toward the Construction of NanotubeBased Gene Delivery Vectors. J. Am. Chem. Soc. 127, 4388-4396 (2005).

246. Pantarotto, D. et al. Immunization with Peptide-Functionalized Carbon Nanotubes Enhances Virus-Specific Neutralizing Antibody Responses. Chem. Biol. 10, 961-966 (2003).

247. Chen, R. J. et al. Noncovalent Functionalization of Carbon Nanotubes for Highly Specific Electronic Biosensors. Proc. Natl. Acad. Sci. 100, 49844989 (2003).

248. Lacerda, L., Bianco, A., Prato, M. \& Kostarelos, K. Carbon nanotubes as nanomedicines: from toxicology to pharmacology. Adv. Drug Deliv. Rev. 58, $1460-70$ (2006). 
249. Fisher, C. et al. Applications and Nanotoxicity of Carbon Nanotubes and Graphene in Biomedicine. J. Nanomater. (2012). doi:10.1155/2012/315185

250. Vales, G., Rubio, L. \& Marcos, R. Genotoxic and cell-transformation effects of multi-walled carbon nanotubes (MWCNT) following in vitro sub-chronic exposures. J. Hazard. Mater. 306, 193-202 (2015).

251. Liu, Y., Zhao, Y., Sun, B. \& Chen, C. Understanding the Toxicity of Carbon Nanotubes. Acc. Chem. Res. 46, 702-713 (2013).

252. Sargent, L. M. et al. Promotion of lung adenocarcinoma following inhalation exposure to multi-walled carbon nanotubes. Part. Fibre Toxicol. 11, (2014).

253. Stella, G. M. Carbon nanotubes and pleural damage: perspectives of nanosafety in the light of asbestos experience. Biointerphases 6, P1-17 (2011).

254. Shvedova, A. A. et al. Mechanisms of carbon nanotube-induced toxicity: Focus on oxidative stress. Toxicol. Appl. Pharmacol. 261, 121-133 (2015).

255. Shvedova, A. A. et al. Inhalation vs. aspiration of single-walled carbon nanotubes in C57BL/6 mice: inflammation, fibrosis, oxidative stress, and mutagenesis. Am. J. Physiol. Lung Cell. Mol. Physiol. 295, L552-65 (2008).

256. Shvedova, A. a et al. Long-term effects of carbon containing engineered nanomaterials and asbestos in the lung: one year postexposure comparisons. Am. J. Physiol. Lung Cell. Mol. Physiol. 306, L170-82 (2014). 
257. Muller, J. et al. Respiratory toxicity of multi-wall carbon nanotubes. Toxicol. Appl. Pharmacol. 207, 221-31 (2005).

258. Shukla, A., Vacek, P. \& Mossman, B. T. Dose-Response Relationships in Expression of Biomarkers of Cell Proliferation in In Vitro Assays and Inhalation Experiments. Nonlinearity Biol. Toxicol. Med. 2, 117-28 (2004).

259. Lohcharoenkal, W. et al. Chronic exposure to carbon nanotubes induces invasion of human mesothelial cells through matrix metalloproteinase-2. ACS Nano 7, 7711-23 (2013).

260. Nagai, H. et al. Diameter and rigidity of multiwalled carbon nanotubes are critical factors in mesothelial injury and carcinogenesis. Proc. Natl. Acad. Sci. U. S. A. 108, E1330-8 (2011).

261. Biju, V. Chemical modifications and bioconjugate reactions of nanomaterials for sensing, imaging, drug delivery and therapy. Chem. Soc. Rev. 43, 744$64(2014)$.

262. Schipper, M. L. et al. A pilot toxicology study of single-walled carbon nanotubes in a small sample of mice. Nat. Nanotechnol. 3, 216-21 (2008).

263. Stueckle, T. a et al. Chronic occupational exposure to arsenic induces carcinogenic gene signaling networks and neoplastic transformation in human lung epithelial cells. Toxicol. Appl. Pharmacol. 261, 204-16 (2012). 
264. Siegrist, K. J. et al. Genotoxicity of multi-walled carbon nanotubes at occupationally relevant doses. Part. Fibre Toxicol. 11, (2014).

265. Nagai, H. \& Toyokuni, S. Differences and similarities between carbon nanotubes and asbestos fibers during mesothelial carcinogenesis: shedding light on fiber entry mechanism. Cancer Sci. 103, 1378-90 (2012).

266. Park, Y.-H., Kim, D., Dai, J. \& Zhang, Z. Human bronchial epithelial BEAS2B cells, an appropriate in vitro model to study heavy metals induced carcinogenesis. Toxicol. Appl. Pharmacol. 287, 240-5 (2015).

267. Wang, Y. et al. Transforming growth factor- $\beta$ regulates the sphere-initiating stem cell-like feature in breast cancer through miRNA-181 and ATM. Oncogene 30, 1470-80 (2011).

268. Pongrakhananon, V. et al. Carbon nanotubes induce apoptosis resistance of human lung epithelial cells through FLICE-inhibitory protein. Toxicol. Sci. 143, 499-511 (2015).

269. Lippert, B. M., Knauer, S. K., Fetz, V., Mann, W. \& Stauber, R. H. Dynamic survivin in head and neck cancer: molecular mechanism and therapeutic potential. Int. J. Cancer 121, 1169-74 (2007).

270. Chakrabarty, A. et al. Trastuzumab-resistant cells rely on a HER2-PI3KFoxO-survivin axis and are sensitive to PI3K inhibitors. Cancer Res. 73, 1190-200 (2013). 
271. Fuessel, S. et al. Chemosensitization of bladder cancer cells by survivindirected antisense oligodeoxynucleotides and siRNA. Cancer Lett. 232, 243-54 (2006).

272. Würl, P. et al. Co-expression of survivin and TERT and risk of tumour-related death in patients with soft-tissue sarcoma. Lancet 359, 943-5 (2002).

273. Laaksonen, T. et al. Failure of MTT as a toxicity testing agent for mesoporous silicon microparticles. Chem. Res. Toxicol. 20, 1913-8 (2007).

274. Liao, K.-H., Lin, Y.-S., Macosko, C. W. \& Haynes, C. L. Cytotoxicity of graphene oxide and graphene in human erythrocytes and skin fibroblasts. ACS Appl. Mater. Interfaces 3, 2607-15 (2011).

275. Han, X. et al. Validation of an LDH assay for assessing nanoparticle toxicity. Toxicology 287, 99-104 (2011).

276. Suska, F., Gretzer, C., Esposito, M., Tengvall, P. \& Thomsen, P. Monocyte viability on titanium and copper coated titanium. Biomaterials 26, 5942-50 (2005).

277. Boutry, S. et al. How to quantify iron in an aqueous or biological matrix: a technical note. Contrast Media Mol. Imaging 4, 299-304 (2009).

278. Boutry, S. et al. Magnetic labeling of non-phagocytic adherent cells with iron oxide nanoparticles: a comprehensive study. Contrast Media Mol. Imaging 3, 223-32 (2008). 
279. Riemer, J., Hoepken, H. H., Czerwinska, H., Robinson, S. R. \& Dringen, R. Colorimetric ferrozine-based assay for the quantitation of iron in cultured cells. Anal. Biochem. 331, 370-5 (2004).

280. Hummel, F. C. \& Willard, F. H. Determination of Iron in Biological Materials The Use of o-Phenanthroline. Ind. Chem. Eng. 13-15 (1938).

281. Roth, H.-C., Schwaminger, S., Fraga García, P., Ritscher, J. \& Berensmeier, S. Oleate coating of iron oxide nanoparticles in aqueous systems: the role of temperature and surfactant concentration. J. Nanoparticle Res. 18, 99 (2016). 


\section{Chapter 2}

\section{Targeting Survivin: Antisense-coupled SPIONs for Targeted Cancer Imaging and Therapy}

*Presented at IEEE EMBS 2014 Micro and Nanotechnology in Medicine Conference, Oahu, HI.

http://emb.citengine.com/event/mnm-2014/paper-details?pdID=2567

${ }^{*}$ Emily C. Despeaux ${ }^{1,2}$, Kelly Pisane ${ }^{2}$, Anand Narayanan ${ }^{1}$, Siera Talbott ${ }^{3}$, Peter M. Gannett ${ }^{1}$, Mohindar Seehra ${ }^{2}$, R. Lloyd Carroll. ${ }^{4}{ }^{1}$ Department of Pharmaceutical Sciences, ${ }^{2}$ Department of Physics, ${ }^{3} W V U$ BioNano Research Facility, and ${ }^{4}$ Department of Chemistry, West Virginia University, Morgantown, WV, 26506. 


\subsection{Abstract}

Superparamagnetic iron oxide nanoparticles (SPIONs) can be used to improve the specificity of cancer drug delivery, thereby reducing the toxicity associated with systemic administration. SPIONs can be easily conjugated to therapeutic agents, including antisense oligonucleotides. Survivin, an anti-apoptotic protein over-expressed in many types of cancer but undetectable in most healthy adult tissues, is an attractive therapeutic target. Increases in survivin expression are correlated with more aggressive cancers, treatment resistance, and greater patient mortality. Antisense agents alone are an effective means of reducing survivin expression in vivo, where they trigger apoptosis specifically in survivin expressing cells. In vivo, however, a platform is needed to deliver antisense agents to tumor cells. SPIONs coated in oleic acid were synthesized by thermal decomposition and coated with a biocompatible polymer, octylamine modified polyacrylic acid before conjugation with survivin antisense DNA. SPION physical properties, including particle size and composition, were characterized at each step of synthesis. A549 (human lung adenocarcinoma) cells, which have high baseline survivin expression, were exposed to DNA-coupled SPIONs for 24 or 48 hours. SPION uptake and localization was followed with confocal and fluorescent microscopy. Survivin expression was quantified with Western blots, while cytotoxicity was assessed with the colorimetric water soluble tetrazolium (WST-1) assay. Our results showed that the SPION platform is biocompatible and capable of delivering functional antisense oligonucleotides to regulate survivin expression; however, significant refinement of the DNA-to-SPION coupling step is needed. Applied clinically, antisense survivin coupled SPIONs can reduce the required dose of, adverse effects from, and resistance to, current cancer chemotherapy regimens. 


\subsection{Introduction}

Many of the systemic toxicities of current cancer chemotherapeutic agents are the result of the drugs' indiscriminate action on all rapidly dividing cells. Drugs that specifically target cancer cells are difficult to design due to the considerable spatial and functional overlap between healthy and malignant cells. One target that is ubiquitous in cancer cells, yet virtually undetectable in healthy cells, is the anti-apoptotic protein survivin. ${ }^{1-3}$ Survivin overexpression has been identified in nearly all types of cancer, including lung adenocarcinoma, ${ }^{4}$ mesothelioma,${ }^{5}$ anaplastic large-cell lymphoma, ${ }^{6}$ osteosarcoma, ${ }^{7}$

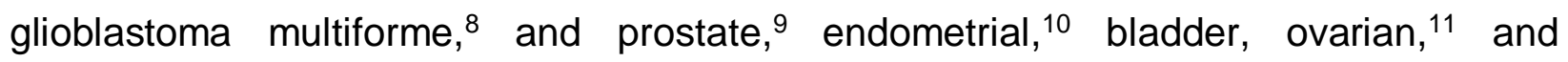
pancreatic $^{12}$ carcinomas. Clinically, high tumor survivin levels correlate with chemotherapy and radiation resistance, increased metastases, and overall greater mortality. 4,7,9,10,13-16 In vitro, antisense oligonucleotides decrease survivin expression and increase sensitivity to chemotherapy and radiation induced apoptosis without affecting the survival of non-survivin expressing cells. ${ }^{1,5,9,10,17-22}$ The specificity of survivin-targeted therapies and their striking in vitro success spawned a number of clinical trials, including YM155, a small-molecule inhibitor of survivin, ${ }^{23}$ a vaccination against a survivin peptide, ${ }^{24}$ and LY2181308, a naked antisense survivin agent administered in saline. ${ }^{25}$ Although none of the tested therapies progressed in their initial states due to formulation issues or practical considerations, they demonstrated the viability of clinically targeting survivin.

Specifically, nucleic acids are challenging to deliver in vivo. Naked nucleic acids are rapidly hydrolyzed upon entering the bloodstream, rendering them inactive. ${ }^{26,27}$ Then, even if a therapeutically relevant concentration of antisense agent is reached in the target 
tissue, naked nucleic acids require coaxing to cross cell membranes. Their dense negative charge is repelled by the negative charge on the surface of cell membranes and in vitro strategies to increase uptake, including liposomes and electric shock, do not translate well to in vivo settings. ${ }^{28,29}$ Structural modifications to the nucleic acid bases have been employed to increase stability in circulation by reducing enzymatic degradation. ${ }^{26,28-31}$ Other delivery strategies have included encapsulation of the nucleic acids in a cationic polymer ${ }^{27-29}$ or $\mathrm{pH}$ sensitive coatings for controlled release. ${ }^{27,31,32}$ Considering the strong in vitro response to survivin antisense agents, and the known limitations to the delivery of naked nucleic acids in vivo, ${ }^{26,28,33}$ a stable, biocompatible antisense delivery system is needed before their clinical potential can be realized. ${ }^{29}$

Superparamagnetic iron oxide nanoparticles (SPIONs) have been widely investigated for use as both drug delivery platforms ${ }^{34,35}$ and MRI contrast agents ${ }^{36}$ and, recently, Ferumoxytol (FeraHeme) has received FDA approval for use a treatment for anemia in patients with chronic kidney disease ${ }^{37}$ SPIONs are readily metabolized to elemental iron in vivo, ${ }^{35,3737}$ making them an attractive platform for diagnostics and therapeutics. Ease of SPION functionalization allow for great versatility and the design of multipurpose particles. ${ }^{35,38}$ SPIONs will accumulate passively in tumors due to leaky vasculature and poor lymph clearance, and active targeting with tumor-specific antibodies or aptamers can further increase particle concentration in target tissue..$^{37-43}$

This work centers on the development of a SPION-based platform for the delivery of survivin antisense oligonucleotides to cancer cells. We anticipate that this platform will 
result in selective toxicity to malignant, survivin expressing cells, leaving healthy, nonsurvivin expressing cells unscathed.

SPIONs coated in oleic acid were synthesized by thermal decomposition ${ }^{44}$ and coated with a biocompatible polymer, octylamine modified polyacrylic acid (Figure 1), ${ }^{45}$ to increase aqueous solubility and provide reactive sites for further functionalization. Polymer coated SPIONs were coupled to 3'- amine-modified survivin antisense ${ }^{1}$ or nontargeting control DNA sequences. ${ }^{46}$ 1-Ethyl-3-(3-dimethylaminopropyl)carbodiimide (EDC) was used to catalyze the formation the 3 '-amide linkage. ${ }^{46}$ SPION physical properties, including particle size and composition, were characterized at each step of synthesis. A549 (human lung adenocarcinoma) cells, which have high baseline survivin expression, ${ }^{47}$ were exposed to DNA-coupled SPIONs for 24 or 48 hours. SPION uptake and localization was followed with confocal and fluorescent microscopy. Survivin expression was quantified with Western blots, while cytotoxicity was assessed with the colorimetric water soluble tetrazolium (WST-1) assay. Our results showed that the SPION platform is biocompatible and capable of delivering functional antisense oligonucleotides to regulate survivin expression; however, significant refinement of the DNA-to-SPION coupling step is needed.

\subsection{Results}

\subsubsection{SPION Synthesis and Characterization.}

Oleic acid coated SPIONs (SPION-OA) were synthesized using thermal decomposition, as previously described. ${ }^{44}$ After washing, the synthesis consistently 
yielded homogenous particles with $7.07 \pm 0.74 \mathrm{~nm}$ SPION cores (Figure 2). SPION-OA readily disperse organic solvents, like hexane and toluene, even at high concentrations, resulting in opaque black solutions. We have previously described the full characterization of the elemental composition and magnetic susceptibility of SPION-OA. ${ }^{61}$

An octylamine modified poly(acrylic) acid (PAA) coating ${ }^{45}$ was added to SPION-OA to allow transfer to aqueous solvents and to serve as a link to additional functionalization. Hydrophobic interactions are expected to drive the octylamine moieties to intercalate with the hydrophobic oleic acid tails, resulting in the formation of a hydrophobic layer encapsulated by the outward-facing carboxylic acids of the PAA backbone. ${ }^{62}$ The newly formed PAA coated SPION-OA (SPION-PAA) were washed, dried, and suspended in basic water $(\mathrm{pH} 9)$ before filtering to remove aggregates; without filtering, SPION-PAA was difficult to disperse and rapidly settled out of solution. Unfortunately, much of the SPION-PAA characterization was carried out before we discovered the importance of filtering the samples.

To verify the addition of the PAA coating, FT-IR was performed on SPION-OA and SPION-PAA. The SPION core is not expected to contribute greatly to the FT-IR spectra, but the interactions of the oleic acid with the core allow for differentiation of SPION-OA from oleic acid alone (Figure $3 \mathrm{~A}$ ). Specifically, oleic acid alone has a strong carboxyl $(\mathrm{C}=\mathrm{O})$ peak at $1750 \mathrm{~cm}^{-1}$. However, the carboxyl $\left(1750 \mathrm{~cm}^{-1}\right)$ peak is very small in SPIONOA, which aligns with the expectation that the oleic acid carboxyl group is binding SPION. The small remaining carboxyl signal may be the result of oleic acid that is not covalently 
bound. ${ }^{61}$ After coating SPIONs with PAA, a small carboxylic acid hydroxyl $(\mathrm{OH})$ peak is present at $\left(3333 \mathrm{~cm}^{-1}\right)$ (Figure $\left.3 \mathrm{~B}\right)$. These hydroxyl groups are critical to further functionalization of the platform. The small size of the peak is expected as the hydroxyl groups are a small percentage of the total structure.

Thermogravimetric analysis (TGA) was used to quantify the mass contributions of each coating in SPION-OA and SPION-PAA, while dynamic light scattering (DLS) was used to determine the contributions of the coatings to the hydrodynamic diameter of the particles (Figure 4). SPION-OA was found to be $20 \%$ oleic acid and $80 \%$ iron oxide, a ratio that remained consistent across samples and synthesis batches (Figure 4, A and C). ${ }^{61}$ SPION-PAA was found to be $75 \%$ PAA, $5 \%$ oleic acid, and $20 \%$ iron oxide (Figure 4, B \& C). SPION-PAA composition was also consistent across batches; however, this measurement was made on unfiltered SPION-PAA, and it is not yet known if the filtered, soluble SPION-PAA will have the same mass composition as the washed but unfiltered particles.

DLS showed that SPION-OA had a hydrodynamic diameter of $11.27 \mathrm{~nm}$, with a narrow size distribution indicative of a homogeneous, well-dispersed sample (Figure 4D). SPIONOA hydrodynamic diameter was $\sim 4 \mathrm{~nm}$ larger than the diameter determined from TEM, which was expected since TEM only allowed measurement of the metallic core while DLS accounted for the iron oxide core and oleic acid coating. SPION-PAA had a hydrodynamic diameter of $43.12 \mathrm{~nm}$ (Figure 4E). Although the DLS histogram shows a narrow size distribution, this measurement was performed on the poorly soluble, unfiltered SPION- 
PAA. Large aggregates of SPION-PAA, visible to the naked eye, are present in nearly all suspensions of unfiltered SPION-PAA; however, these aggregates often settle out or float, which would result in less of an impact on the DLS measurement.

\subsubsection{Coupling DNA to SPION-PAA}

SPION-PAA forms the base of our delivery platform, with outward-facing carboxylic acids from the PAA providing covalent attachment sites for amine-terminal ligands like our survivin antisense (SASO) and non-targeting control (NTC) oligonucleotides. Our single-stranded DNA was synthesized with a $3^{\prime} \mathrm{C}_{7}-\mathrm{NH}_{2}$ to allow for coupling via carbodiimide chemistry. Our intent was to use 1-Ethyl-3-(3dimethylaminopropyl)carbodiimide (EDC) to drive the reaction, as $E D C /$ carbodiimide chemistry is widely utilized for the formation of amide bonds, particularly between biological molecules. ${ }^{45,63,64,50,54}$ However, our coupling success varied dramatically, often varying by a factor of ten between reactions performed in parallel from identical starting materials. While a definitive procedure for this coupling application was not successfully discerned in the course of this work, a number of variables have been identified as targets for reaction optimization.

Despite the issues with DNA coupling consistency, some characterization of SPIONDNA was carried out. TEM was used to examine the SPION core of a batch of SASOSPION as well as a sample of the SPION-OA from which they were derived (Figure 5, A $\&$ B). Although the additional coating layers are not expected to change the core size, the core diameter of SPION-OA $(7.07 \pm 0.74 \mathrm{~nm})$ was found to be $2 \mathrm{~nm}$ smaller than the 
average core diameter of SASO-SPION $(9.01 \pm 0.72 \mathrm{~nm})$. The difference may be due, at least in part, to the small sample size for the SASO-SPION. SASO-SPION was prepared from a very dilute solution and particles were sparse when imaged.

DLS showed that SASO-SPION had a hydrodynamic diameter of $50.58 \mathrm{~nm}$ (Figure $5 \mathrm{C}$ ). Thus, the DNA makes up a small portion of the final SPION diameter (Figure 6). It is interesting to note that even though the amount of DNA coupling to the SPIONs was not consistent, the change in particle diameter when DNA was added is in line with what would be expected if the SASO covalently bound (Table 1). It is not clear at this point is if the biologically active DNA is the covalently bound portion or is, perhaps, made up of an additional layer of DNA attached through electrostatic interactions.

\subsubsection{SASO-SPION Decrease Survivin Expression and Reduce Survival in A549 Cells, a High Survivin Human Lung Cancer Cell Line.}

Although the reaction for coupling DNA to SPIONs is still in need of refinement, our preliminary in vitro results that suggest our SPION platform is not inherently toxic to cells and support the viability of this platform as a delivery system for antisense oligonucleotides.

It is important to note that the following results were obtained by using multiple batches of SPION-DNA. Although every attempt has been made to standardize the dosing measures and presentation, the batch-to-batch variation of the current DNA coupling procedure is such that that results from different batches are not directly comparable. 
Dosing evolution is addressed in the discussion, while dosing explanations are included in the procedure and results for each experiment. However, the results are, as a whole, supportive of the hypothesis that this SPION platform has potential as a drug delivery agent. This data is meant to serve as proof-of concept and will be presented with appropriate details on the SPIONs used in each study. In the future, once the DNA coupling/formulation issue has been addressed, in vivo studies will be repeated and expanded to determine the actual magnitude and time course of the biological effects.

Early in the formulation development process, confocal microscopy was used to verify that SPION-DNA could enter our target A549 lung adenocarcinoma cells (Figures 7 and 8A). For this study, SPION-PAA was coupled to a fluorescein-labeled antisense survivin oligonucleotide. Following a 24 hour incubation, cells were thoroughly washed and fixed before staining with DAPI and FM4-64FX cell membrane stain. 3D renderings confirmed that SPIONs were internalized and were within the cytoplasmic compartment. Since this was an early-stage study, the main purpose was to show that the SPIONs could enter cells, which was accomplished. In addition, the determination that the SPIONs were in the cytoplasm supports the use of this system in hindering protein production via antisense oligonucleotide interference with cytoplasmic mRNA.

After determining that the SPIONs were entering the cells, we briefly investigated the mechanism of entry (Figure 8B). A549 cells were again incubated with fluorescently labeled SASO-SPION for 24 hours and cells were stained with DAPI and either a cell membrane stain (Cell Mask Orange) or a lysosome tracking dye (Lysotracker Deep Red). 
Examination of fixed cells with fluorescent microscopy confirmed that SPIONs were entering the cells. Co-localization of the lysosome/membrane dyes with the SPIONs fluorescent tag suggests that the SPIONs are within vesicles, with many notable localization to the peri-nuclear region.

While SPION internalization is likely a necessary step in delivering the therapeutic DNA to the cells, the lysosomal/vesicular localization of internalized SPIONs was concerning. ${ }^{27,29}$ Cytoplasmic localization of the SPIONs, or at least the therapeutic antisense ligands, is required for the downregulation of protein production through the intended mechanism. ${ }^{27-29}$ Since the goal of this specific SPION formulation is induce or sensitize cells to apoptosis through decreased survivin expression, changes in cell viability and survivin expression were then assessed.

For assessment of the toxic and/or therapeutic effects of DNA coupled SPIONs, SPIONs were dosed by DNA concentration. DNA concentration was chosen because it could be measured using non-destructive, reproducible methods and it allowed for comparison to previously reported studies on our chosen SASO. Ideally iron concentration would also be accounted for, perhaps in the form of a consistent relationship between iron and DNA concentrations. Unfortunately that was not possible in this study.

To test our hypothesis was that SASO-SPIONs would contribute to cancer cell death by decreasing survivin expression, A549 cells were exposed to SASO- or NTC-SPION 
(1000 nM) for 24 hours and survivin was detected by Western blot (Figure 9A). Our initial studies used a high DNA concentration (1000 nM) so that an effect, if present, would be readily apparent. We found that after 24 hours, SASO-SPIONS significantly reduced survivin expression to $66.14 \%$ ( $\pm 15.11 \%$ ) of the untreated control, while NTC-SPIONs resulted in survivin expression that was $110.87 \%( \pm 9.67 \%)$ of the untreated control. Olie, et al. reported that the same SASO sequence, at 400 and $600 \mathrm{nM}$, reduced survivin mRNA to $35 \%$ of the untreated control when introduced to A549 cells with lipofectin. ${ }^{1}$

While decreases in cell survivin expression have been correlated to increased sensitivity to chemotherapy drugs and radiation, decreasing an anti-apoptotic mediator does not inherently trigger apoptosis. Thus, the cytotoxic effects of SASO- and NTCSPIONs on A549 cells were assessed following 24 and 48 hour exposures.

A lactate dehydrogenase (LDH) release assay (results not shown) was used to quantify cell necrosis and late-stage apoptosis, as LDH is released into the surrounded media when cells lyse. Metabolic viability of remaining live cells was determined from using WST-1, a colorimetric assay where functional mitochondria convert a formazan dye to a water soluble tetrazolium salt and the color change is proportional to the number of live cells. Since the LDH assay is performed on used cell culture media and WST is performed on the cells themselves, both assays were performed on the same exposed cells at the same time. 
Although LDH is frequently used as a measure of cytotoxicity in SPION or nanoparticle-exposed cells, it is not always an appropriate choice for the quantification of cell death. ${ }^{65}$ In our case, many of our LDH results showed that treated cells experienced much less toxicity (cell lysis) than untreated controls even though the WST results (or simply viewing the wells through a microscope) indicated the opposite. It turns out that once LDH is released it has a half-life of 9 hours in solution. As a consequence, it is not well suited to capture cell death unless it is occurring relatively close the time of the assay. If the tested agent is causing rapid cell death or growth restriction, the LDH released from the dying cells will not be picked up by the assay. Since cells have a small level of baseline $\mathrm{LDH}$ release, using LDH as the only measure of cytotoxicity will show a well with no cells as "healthier" than a control well with a healthy cell population.

Metabolic viability of the cells was assessed after 24 and 48 hour SPION exposures. After 24 hours, no significant changes in viability were noted at any dose of SASO- or NTC-SPION (Figure 9B). However, our 48 hour incubation (Figure 9, C \& D) revealed that the effects of the SASO-SPIONs were strongly batch dependent. In comparison, no changes in viability were noted at any dose, or for any batch, of NTC-SPION, indicating that they are innocuous. The two coupling batches tested in Figure $9 C \& D$ were prepared from the same starting materials and prepared and tested in parallel. However, UV-vis analysis immediately after coupling suggested that batch 1 (Figure 9C) had twice as much DNA per SPION as batch 2 (Figure 9D). Although the batch-to-batch variation was high, replicates within each batch were remarkably consistent $( \pm 4 \%)$. This SASO sequence, when introduced to A549 cells by lipofectin, reduced cell viability to 50\% (300 nM SASO) 
and $10 \%$ (600 nM SASO) after 72 hours. ${ }^{1}$ Once our SPION-DNA coupling procedure has been optimized, assessing cell viability at 72 hours will allow us to compare the efficacy of our delivery platform to the current delivery standard.

While more studies are needed to fully understand the does- and time-dependent effects of the SASO-SPIONs, this batch dependence does support the biocompatibility of the SPIONs. Specifically, the less effective batch had less DNA per SPION, meaning that significantly more poorly-coupled SPIONs were required to achieve each DNA dose. Additionally, no significant toxicity is noted with NTC-SPIONs at any dose or from any batch.

\subsection{Discussion}

In this work, we demonstrated that a biocompatible SPION scaffold can be reliably synthesized and coated. The functionalization of our SPION platform has been inconsistent; however, our preliminary data supports the use of this system to deliver antisense oligonucleotides to alter cell behavior. Specifically we have chosen to target the anti-apoptotic mediator survivin, which is heavily expressed in cancer cells but virtually undetectable in healthy tissue. ${ }^{1-4,7,9,10,13-16}$

The overexpression of survivin in malignant tissue has been well established, as have the benefits of reducing survivin expression in vitro., $1,9,9,10,17$ Unfortunately there has been great difficulty in translating those in vitro successes to clinically useful treatments. One major obstacle in clinical translation has been the difficulty of delivering therapeutic oligonucleotides in vivo. ${ }^{26,28-31}$ Thus we proposed the use of a SPION-based platform to 
chaperone the nucleic acids, in our case an antisense oligonucleotide, to their in vivo destinations.

The magnetic properties of SPIONs make them particularly appealing for clinical applications. In addition to delivering therapeutic agents, SPIONs can act as MRI contrast agents, outlining a tumor or metastasis. Their strong response in magnetic fields means that a low concentration of SPIONs is needed to detect a lesion. The use of superparamagnetic particles, whose magnetism disappears when the magnetic field is removed, reduces the risk of agglomerations forming in small vessels. ${ }^{66}$

SPIONs are widely regarded as biocompatible; however, there is still some debate as to their safety for use in humans. ${ }^{34,35,66-68}$ Lack of standardization in the reporting of nanoparticle characterization, dosing, toxicity measures, etc. makes it difficult to draw conclusions about the safety of various individual SPION formulations. ${ }^{39,54}$ However, general parameters contributing to nanoparticle biocompatibility have been delineated and new formulations can be designed within these parameters to enhance both safety and biological efficacy. For instance, particle diameters between 10 and $100 \mathrm{~nm}$ increase circulation time by minimizing clearance by both the renal and the reticuloendothelial systems. ${ }^{34,35}$ Additionally, a diameter of less than $150 \mathrm{~nm}$ is needed to extravasate. ${ }^{34}$ Since SASO-SPION, our therapeutically active formulation, is $50.28 \mathrm{~nm}$, it is the ideal size to extravasate easily as well as to maximize its half-life in circulation.

Typically SPIONs in circulation interact with plasma proteins, which reduces their bioavailability. ${ }^{68-71}$ However, the negative surface charge of the SASO-SPIONs, resulting 
from the highly-negatively charged DNA backbones, will help to minimize these interactions. Additionally, negatively charged particles will undergo less renal excretion since they will be repelled by the similarly negatively charged glomerular basement membrane. ${ }^{35}$ Decreased renal filtration will enhance circulation time and lessened SPION interactions with the glomerular basement membrane will reduce kidney toxicity.

SPION biocompatibility, including toxicity, half-life, and eventual uptake by target tissues, is heavily influenced by the particles coating. ${ }^{37}$ Our freshly synthesized SPIONs are coated with a layer of oleic acid, which serves as a surfactant to separate the particles during synthesis. ${ }^{44} \mathrm{~A}$ water-soluble coating is needed for the platform to function as a therapeutic, and this coating often serves as the attachment point for therapeutic and targeting ligands. Commonly used coatings include poly(acrylic) acid, ${ }^{45,72,73}$ poly(ethylene) glycol (PEG), ${ }^{40,41,43}$ or dextran, ${ }^{51,74}$ all of which are hydrophilic and accepted as biocompatible. ${ }^{37,38}$ Therapeutic and targeting ligands, including nucleic acids, antibodies, and aptamers, can the conjugated to the SPION coating. Covalent attachment of these ligands, in the form of EDC-catalyzed amide bond formation linking amine-terminal biological molecules to surface carboxylic acid moieties, is common but the procedures for doing so are not well defined.

Carbodiimides activate carboxylate anions to an O-acylurea intermediate in the condensation reaction between amines and carboxylic acids. Then the amine can attack the intermediate to form an amide or another carboxylate can attack to generate an anhydride, which is then attacked by the amine. ${ }^{75}$ EDC is popular for these reactions 
because it is water soluble, the excess reagent is easy to remove, and the reaction can take place at room temperature. ${ }^{50,54,55}$ Despite its popularity, the mechanics and necessary parameters of EDC-driven amide formation are not well understood, ${ }^{63,54,55,76}$ leading to dramatic variations in reported procedures ${ }^{51,53,49,76-78}$ and, frequently, low coupling efficiencies. ${ }^{63,54,55,76}$ Further complicating matters, EDC initially gained wide acceptance for its ability to form amide bonds on surfaces, and adaptation of the surface modification protocol for use with nanoparticles in solution requires additional consideration. ${ }^{55,76}$ For example, in many cases excess EDC causes nanoparticle aggregation or precipitation, ${ }^{54,77,79}$ possibly because the positively charged activated EDC molecules neutralize the negative charges of the carboxyl groups that are keeping the particles suspended. ${ }^{54}$

The use of EDC for both surface and nanoparticle modification requires careful consideration of the reaction conditions. Controlling reaction $\mathrm{pH}$ is critical, as only the protonated form of EDC participates in reactions. With a pKa of 3.5, EDC is most active in acidic conditions $(\mathrm{pH}<4) .50$ In our SPION-DNA coupling reaction, the pKa of the carboxylic acid and amine groups to be linked must also be considered. The timing is also critical, as EDC rapidly hydrolyzes in aqueous solutions. ${ }^{50,75}$ Given the proclivity of EDC to hydrolyze in the presence of water, the use of properly stored reagent and freshly prepared EDC solutions is imperative. This could contribute to the reported inconsistencies in EDC efficiency and reaction concentrations. EDC is readily available in large quantities, particularly relative to the amounts needed for small-scale reactions, and the water sensitivity of the reagent would allow for degradation with time, repeated 
container opening, or improper storage conditions. As a result, larger quantities of reagent would be needed the longer the container is open.

Despite the challenges in obtaining SASO-SPIONs with consistent DNA coatings, we found that our SASO-SPIONs had a measurable effect on cell viability. Although this is what the platform was designed for, it led to some questions as to the mechanism of SPION entry into the cell and how the SASO was ending up in the cytoplasm. Our imaging results suggested that the SASO-SPIONs were internalized via endosomes, which tend to acidify and degrade their contents. ${ }^{29}$ The successful use of SPIONs as an iron source for patients with iron deficiency anemia would also support the theory that SPIONs are taken up and degraded. ${ }^{35,37}$ Although other studies have also visualized SPION ${ }^{80}$ and antisense-loaded gold nanoparticles uptake through endocytosis, ${ }^{81-83}$ it is possible that SPIONs are internalized via multiple routes. Another possibility is that it is not the covalently bound DNA that is responsible for the changes in cell behavior; rather, a layer of non-covalently bound DNA may form on the particle surface through electrostatic interactions. This weakly bound DNA would be freed in response to environmental changes, like the drop in $\mathrm{pH}$ encountered in the endocytic vesicles, and the free DNA could migrate into the cytoplasm. ${ }^{37,84,85}$

To fully exploit either the drug delivery or imaging potential of our particles, we first need to demonstrate selective uptake by the target cells. Our present uptake studies demonstrated that SPIONs entered a survivin expressing cell line. The current SPION formulation lacks active targeting ligands and is expected to passively distribute throughout the body, with a preference for tumors or areas of inflammation due to the 
leaky vasculature in those regions. As such, the potential for high levels of non-specific SPION accumulation, particularly in hepatic and splenic tissue, remains a concern. To these ends, a method for actively targeting the SPIONs specifically to survivin-expressing cells would improve their clinical utility. Although passive targeting may allow SPIONs to accumulate in some tumors as is, specific targeting will increase their effectiveness.

Active targeting of the SPIONs would also allow us to utilize the oleic acid layer that exists between the SPION core and the polymer coating as drug reservoir. Lipophilic drugs, such as doxorubicin, can be loaded into the oleic acid to allow for targeted delivery of high drug concentrations with decreased risk of systemic toxicity. Many drugs are known to act synergistically with the sensitization from decreased survivin levels to combat resistant cancers ${ }^{1,15}$ and delivering such chemotherapeutic agents with the SPION:DNA platform could improve outcomes in patients with aggressive or resistant malignancies.

Although more studies, both in vitro and in vivo, are needed to sufficiently demonstrate the safety of our SPION platform, these initial studies suggest that low doses of our SASO-SPIONs decrease survival of survivin expressing cells. If utilized to their full potential, our SPIONs are capable of dramatically improving cancer diagnostics and therapeutics. Specifically, their multi-functional nature allows a single well-designed SPION to be used for cancer diagnostics, treatment, and non-invasive monitoring of response to therapy. 


\subsection{Materials and Methods}

2.5.1 SPION Synthesis. Oleic acid coated SPIONs were synthesized following a previously described thermal decomposition method. ${ }^{44}$ Octyl ether $(10 \mathrm{ml}, 0.033 \mathrm{mmol})$ (Sigma-Aldrich, Milwaukee, WI) and oleic acid (1.43 ml, $4.56 \mathrm{mmol}$ ) (Santa Cruz, Dallas, TX) were heated to $100^{\circ} \mathrm{C}$. Iron pentacarbonyl $(0.2 \mathrm{ml}, 1.52 \mathrm{mmol})$ (Sigma-Aldrich, Milwaukee, WI) was added and the solution was refluxed for 1 hour (solution turned black). After cooling to room temperature, trimethyl amine $\mathrm{N}$-oxide $(0.34 \mathrm{~g}, 4.56 \mathrm{mmol})$ (Sigma-Aldrich, Milwaukee, $\mathrm{WI}$ ) was added and temperature was increased to $130^{\circ} \mathrm{C}$ for 2 hours, at which point the solution was brown. The solution was then heated to reflux for 1 hour. The resulting black solution was allowed to cool to room temperature.

To remove excess oleic acid, small aliquots of reaction mixture were transferred to a beaker over a strong magnet. Ethanol (200 proof) (Sigma-Aldrich, Milwaukee, WI) was added until the SPIONs precipitated. Liquid was decanted and the oleic acid coated SPIONs (SPION-OA) were suspended in toluene $(500 \mu \mathrm{l})$. Each aliquot was washed a total of three times and the final product was collected in toluene.

\subsubsection{Octylamine-modified Polyacrylic Acid (PAA) Synthesis. ${ }^{45}$ 1-Ethyl-3-(3-} dimethylaminopropyl)carbodiimide (EDC) (5.33g, $28 \mathrm{mmol}$ ) (Sigma-Aldrich, Milwaukee, WI) was added to $1800 \mathrm{MW}$ polyacrylic acid $(5.0 \mathrm{~g}, 70 \mathrm{mmol})$ in DMF (70 ml) (Alfa Aesar, Ward Hill, MA) and stirred for 30 minutes. Octylamine (4.6 ml, $28 \mathrm{mmol})$ (Sigma-Aldrich, Milwaukee, WI) was added and stirred overnight at room temperature. Solvent was removed under vacuum. Distilled water was added and the resulting precipitate was 
isolated by centrifugation (300g, 3h). Aqueous $\mathrm{NaOH}(1 \mathrm{M})$ (Fisher Scientific, Pittsburgh, PA) was added and the mixture shaken overnight. Solution was washed with ethyl acetate $(3 \times 20 \mathrm{ml})$. Dilute $\mathrm{HCl}(10 \% \mathrm{HCl})$ (Fisher Scientific, Pittsburgh, PA) was added to the aqueous layer until the $\mathrm{pH}$ was less than 5 to give a white precipitate. Precipitate was collected following centrifugation $(300 \mathrm{~g}, 3 \mathrm{~h})$ and dried. $\mathrm{NaOH}(0.1 \mathrm{M})$ was used to neutralize dry product. Final product was dried over $\mathrm{P}_{2} \mathrm{O}_{5}$ and a PerkinElmer Spectrum One FT-IR spectrometer was used in transmission mode under ambient conditions to verify the structure of the modified polymer.

Octylamine-modified PAA (50 mg) was added to dry SPIONs (10 mg) in chloroform (5 ml) (Fisher Scientific, Pittsburgh, PA) and methanol (3 ml) (Fisher Scientific, Pittsburgh, PA). The mixture was sonicated for 3 hours and dried under vacuum. Basic water $(\mathrm{pH} 9)$ was added and the solution was filtered through $0.2 \mu \mathrm{M}$ PVDF centrifuge filters (VWR, Radnor, PA) to remove aggregates. Suspended particles were dialyzed with distilled water in Amicon Ultra 15 100K molecular weight cutoff (MWCO) filters (Millipore, Billerica, MA). PAA coated SPIONs (SPION-PAA) were collected and diluted with distilled water. FT-IR of dried PAA-SPIONs was used to verify that the coupling had occurred.

2.5.3 Oligonucleotide Synthesis and Purification. Oligonucleotides were prepared using $1 \mu \mathrm{mol}$ 3'-PT-Amino-Modifier C3 CPG column (Glen Research, Sterling, VA) on an ABI-394 DNA and RNA Synthesizer (Applied Biosciences Incorporated, Foster City, CA). Single-stranded survivin antisense ( $3^{\prime}-\mathrm{C}_{6}$-amino link - GTT CCT CGA CCT TCC GAC CC $\left.-5^{\prime}\right)^{1}$ or non-targeting control (NTC) $\left(3^{\prime}-\mathrm{C}_{6}\right.$-amino link - TTT CCT TTG TGA TCT 
TGC CCT $\left.-5^{\prime}\right)^{48}$ DNA was prepared with and without a 5' fluorescein (Glen Research, Sterling, VA). Concentrated ammonium hydroxide (28-30\%, $1 \mathrm{ml}$ ) (Sigma-Aldrich, Milwaukee, WI) was used to cleave the oligonucleotides from the column. Cleaved oligonucleotides were incubated at $55^{\circ} \mathrm{C}$ for 15 hours and then dried under vacuum. Oligonucleotides were purified with fast protein liquid chromatography using a Waters 510 pump, a Waters 2487 Dual $\lambda$ absorbance detector, and a Bio-Rad TSK DEAE-5-PW column and a linear gradient (10-70\% B over 60 min, Buffer A: $10 \mathrm{mM} \mathrm{NaOH}, \mathrm{pH} 11.8$, Buffer B: $10 \mathrm{mM} \mathrm{NaOH}, 1 \mathrm{mM} \mathrm{NaCl}$, pH 11.8, flow rate $6 \mathrm{ml} / \mathrm{min}$, UV detection, $260 \mathrm{~nm}$ ). Reverse phase Waters Sep-Pack (C-18) (Waters, Milford, MA) cartridges were used for desalting following purification. Absorbance of diluted oligonucleotide solutions was measured on a Beckman DU-600 spectrophotometer (Beckman Coulter Inc., Brea, CA) at 260 and $280 \mathrm{~nm}$.

2.5.4 Oligonucleotide-SPION Coupling. SPION-DNA coupling was inconsistent and the procedures were modified throughout this work with the goal of improving coupling efficiency and reproducibility. No finalized, reproducible coupling parameters were identified, which has led to the inclusion of results from SPIONs coupled under varying conditions. While this is not ideal, the ability to obtain optimistic results even with formulation difficulties supports the potential of this platform. The general procedure for oligonucleotide-SPION coupling is outlined below, followed by notes on procedural changes. The reaction, and possible alternatives, are discussed in greater detail in the results and discussion sections. 
EDC (19 mg, $100 \mathrm{mmol})$ was added to dry SPION-PAA (1 ml, $1 \mathrm{mg})$. Dry DNA (SASO or NTC) (1.5 mg, 40 OD) was dissolved in MilliQ water $(500 \mathrm{~mL})$ and added to the mixture. Additional MilliQ water was added to bring the total volume to $1 \mathrm{ml}$. Mixture was heated at $50^{\circ} \mathrm{C}$ for 3 hours, with vortexing every 15 minutes. Excess DNA was removed by filtration through a Vivaspin 2100 kDa Molecular Weight Cut-off Filter (10 min, $250 \mathrm{~g}$ ) (Vivaproducts, Littleton, MA) using distilled water to wash three times. Washed SPION:DNA was collected in distilled water. The absorbance of SPION solutions at 260 and $280 \mathrm{~nm}$ was measured on a Beckman DU-600 spectrophotometer (Beckman Coulter Inc., Brea, CA) to quantify DNA coupled to SPION.

A number of reaction conditions were modified, at various points, to optimize the reaction. Due to timing and logistical constraints, this analysis was not as thorough or methodical as was needed. One major challenge was that the freshness of the EDC had a strong impact on the reaction, primarily in the form of fresh EDC causing rapid particle precipitation at concentrations similar to, as well as several times less than, those used with older EDC. The difference was so dramatic that the use of fresh EDC nearly negated any prior work on this reaction. SPION-PAA concentration was held constant while EDC concentrations from 0.01 to $19 \mathrm{mM}$ were tried, and DNA concentration was varied from 10 to 100 OD.

Initially, the reaction was performed by adding EDC (powder) to dry DNA, then adding dry SPION-PAA and MilliQ water. Dry SPION-PAA did not disperse well in the solution and attempts to improve its solubility included adding SPION-PAA in a wellsonicated solution of MilliQ water and, later, slightly basic milliQ water. While increasing the $\mathrm{pH}$ of the solution did help to keep the SPIONs in solution, it did not enhance the 
overall reaction efficiency, probably due to inactivating the EDC. ${ }^{49,50}$ To address the $\mathrm{pH}$ sensitivity of EDC, the reaction was next attempted in MES buffer $(25 \mathrm{mM})$ at $\mathrm{pH} 6.0 .{ }^{51-}$ 53,49 However, this reintroduced the SPION-PAA solubility issue.

The reaction time and temperature were also modified throughout this work because the initial conditions frequently resulted in sample destruction. That is, if SPIONs settled at all during the 3 hour incubation at $50{ }^{\circ} \mathrm{C}$, they would decompose and stick to the bottom of the reaction vessel. After consulting additional protocols, the incubation was carried out at room temperature with constant gentle agitation. ${ }^{50,52,54-56}$ As EDC decomposes within the first 15 minutes of the reaction in an aqueous solvent, ${ }^{50}$ so the reaction time was reduced to 1 hour. To encourage SPION suspension throughout the entirety of the reaction, some trials were conducted in an ice bath to allow for continuous sonication.

2.5.5 Transmission Electron Microscopy. A drop of dilute SPION solution was placed on a carbon coated 200-mesh copper grid (Ted Pella Inc, Redding, CA) and allowed to dry at room temperature. A JOEL JEM-2100 transmission electron microscope (Peabody, MA) with an accelerating voltage of $200 \mathrm{kV}$ was used. NIH Image $\mathrm{J}$ software (http://rsbweb.nih.gov/ij/) was used to analyze images for size of iron oxide core.

2.5.6 Dynamic Light Scattering. SPION hydrodynamic diameter was measured in toluene (SPION-OA) or water (all other SPION solutions) with dynamic light scattering (DLS) at a scattering angle of $90^{\circ}$ at $25^{\circ} \mathrm{C}$ using a Malvern Zetasizer Nano (Malvern, UK). 
2.5.7 Thermogravimetric Analysis. Thermogravimetric analysis (TGA) was carried out for powder samples $(3-10 \mathrm{mg})$ from 20 to $700{ }^{\circ} \mathrm{C}$ with a heating rate of $5^{\circ} \mathrm{C} / \mathrm{min}$ in flowing $\mathrm{N}_{2}$ gas using a TA Instruments Model Q50 (New Castle, DE).

2.5.8 Inductively Coupled Plasma (ICP). ICP-OES was used to determine the iron concentration of SPION solutions. SPION solutions were sonicated in $\mathrm{HNO}_{3}(3 \mathrm{ml}, 3.47$ M) overnight. Samples were diluted with water to $50 \mathrm{ml}$ final volume prior to analysis. Analysis was conducted on a Perkin Elmer Optima 7300 V ICP-OES by the West Virginia University National Research Center for Coal and Energy. All samples were prepared in replicates of 6 , and the mean and standard deviation of the sets were calculated.

2.5.9 General Cell Culture. A549 (human lung adenocarcinoma) cells were maintained in 5\% DMEM (Sigma-Aldrich, Milwaukee, WI) supplemented with $5 \%$ heat inactivated FBS (Sigma-Aldrich, Milwaukee, WI), 1\% Penicillin/Streptomycin (Sigma-Aldrich, Milwaukee, $\mathrm{WI}$ ), and $5 \% \mathrm{~L}$-glutamine. Cells were incubated at $37^{\circ} \mathrm{C}$ in a $5 \% \mathrm{CO}_{2}$ environment. Cells were washed with DPBS (Thermo Scientific HyClone, Logan, UT), trypsinized (SAFC Biosciences, Lenexa, KS), and stained with Trypan Blue (Thermo Scientific HyClone, Logan, UT) prior to counting. Cell viability was measured with Cell Proliferation Reagent WST-1 (Roche, Indianapolis, Indiana). Cytotoxicity was measured with Cytotoxicity Detection Kit Plus (LDH) (Promega, Madison, WI). Absorbance measurements were made with a Biotek Epoch Microplate Spectrophotometer (Winooski, VT). 
Note on SPION dosing for in vitro experiments: SPION-DNA doses were initially calculated relative to mass. Later, attempts were made to dose SPION-DNA by iron concentration to deliver similar numbers of SPIONs to each sample, regardless of the ligands. Challenges in determining the iron concentration of small sample volumes in a timely manner led to the use of DNA concentration as the primary dosing measuring. As the therapeutic ligand, the DNA concentration is the measure that best relates to the literature. However, an ideal dosing measure would include both iron concentration and DNA concentration.

2.5.10 Cell Cytotoxicity. For viability studies, SPION-DNA doses were calculated to deliver specified DNA concentrations, which theoretically would overcome the differences in DNA coupling efficiencies. A549 were plated at a density $5 \times 10^{3}$ cells/well in a 96 well plate. DMEM (10\% FBS) $(200 \mu \mathrm{L})$ was added to all wells. Cells were allowed to adhere overnight and media was replaced with $150 \mathrm{ul}$ fresh media plus a total of $50 \mu \mathrm{l}$ of SPIONDNA in PBS $(30,50,80,100,200,500,500,1000 \text { nM DNA })^{1,57}$ and PBS. As nanoparticle interference with many toxicity assays is not well understood and particle interference has been reported under some conditions, ${ }^{58-60}$ cell-free substance controls were set up in the same manner, using the highest dose of SPION-DNA being tested, to ensure SPIONs were not interfering with the assay. All sample sets were run in at least triplicate. Cells were incubated with SPIONs for 24 or 48 hours, after which the plate was placed on a bar magnet for 2 minutes to draw SPIONs to bottom of dish. Supernatant (100 $\mu$ l) from each well was transferred to a clean 96 well plate and freshly prepared LDH assay reaction mixture $(100 \mu \mathrm{L})$ was added to each well. The plate was incubated for 15 minutes 
at room temperature $\left(20^{\circ} \mathrm{C}\right)$. Absorbance was measured at 490 and $620 \mathrm{~nm}$. The cytotoxicity of cells was calculated relative to unexposed cells (all absorbance values used in calculations were at 490 nm): $\frac{\left.(\text { sample abs })-(\text { blank abs })-\left(\frac{\text { experimental concentration }}{\text { substance control concentration }}\right)\right) * \text { substance control abs }}{(\text { untreated control abs })-(\text { blank abs })} \times 100 \%$.

2.5.11 Cell Metabolic Viability. For viability studies, SPION-DNA doses were calculated to deliver specified DNA concentrations, which theoretically would overcome the differences in DNA coupling efficiencies. A549 were plated at a density $5 \times 10^{3}$ cells/well in a 96 well plate. DMEM (10\% FBS) $(200 \mu \mathrm{L})$ was added to all wells. Cells were allowed to adhere overnight and media was replaced with $150 \mathrm{ul}$ fresh media plus a total of $50 \mu \mathrm{l}$ of SPION-DNA in PBS $(30,50,80,100,200,500,500,1000 \text { nM DNA })^{1,57}$ and PBS. As nanoparticle interference with many toxicity assays is not well understood and particle interference has been reported under some conditions, ${ }^{58-60}$ cell-free substance controls were set up in the same manner, using the highest dose of SPION-DNA being tested, to ensure SPIONs were not interfering with the assay. All sample sets were run in at least triplicate. Cells were incubated with SPIONs for 24 or 48 hours, after which media was removed for use in cell cytotoxicity assays, as described above, and remaining adherent cells were washed with PBS $(x 3,100 \mathrm{uL})$ to remove unbound SPIONs. Fresh media (10\% FBS, $100 \mu \mathrm{L})$ and WST-1 reagent $(10 \mu \mathrm{L})$ were added to each well. Absorbance at 450 and $630 \mathrm{~nm}$ was measured after 90 minute incubation at $37^{\circ} \mathrm{C}$. Cell viability was calculated relative to untreated cells with the following equation (all absorbance values used in calculations were at $450 \mathrm{~nm}): \frac{(\text { sample abs })-(\text { blank abs })}{(\text { untreated control abs })-(\text { blank abs })}$. 
2.5.12 Western Blots. A549 cells were incubated with SASO-SPION or SPION-NTC $(0$ or $1000 \mathrm{nM}$ DNA) for 24 hours. The media was removed and cells were washed three times with PBS. Cells were lysed with a lysis buffer $(50 \mathrm{mM}$ Tris- $\mathrm{HCl}, \mathrm{pH} 8.0$ (SigmaAldrich, Milwaukee, WI), 0.1\% wt/vol SDS (Sigma-Aldrich, Milwaukee, WI), 1\% wt/vol glycerol (Sigma-Aldrich, Milwaukee, WI)) and total protein content was measured with Micro BCA kits (Thermo Scientific, Rockford, IL). A total of $60 \mu \mathrm{g}$ protein was loaded into each lane of a tray for SDS-PAGE with $10 \% \mathrm{wt} / \mathrm{vol}$ separating gel and $5 \% \mathrm{wt} / \mathrm{vol}$ stacking gel. Protein was transferred to a polyvinyliene fluoride (PVDF) membrane (30 mA, 2 hours) (Sigma-Aldrich, Milwaukee, WI), which was blocked for 3 hours with a blocking solutions ( $1 \%$ wt/vol bovine serum albumin, 0.05\% Tween-20 (Sigma-Aldrich, Milwaukee, WI), $0.9 \% \mathrm{NaCl}, 0.1 \mathrm{M}$ PBS pH 7.2). Rabbit anti-survivin antibody (1:200, v/v) (SigmaAldrich, Milwaukee, WI) was used to hybridize protein overnight at $4^{\circ} \mathrm{C}$. The membrane was then rinsed with PBS and hybridized with HRP conjugated goat anti-rabbit secondary antibody $(1: 1000, v / v)\left(\right.$ Sigma-Aldrich, Milwaukee, WI) for 2 hours at $37^{\circ} \mathrm{C}$. The signal was detected using ECL western blotting detection reagents and images were acquired on an ImageMaster VDS-CL (Amersham Biosciences, Pittsburgh, PA).

2.5.13 Confocal Microscopy. SPIONs doses in this experiment were based on particle mass, with SPION-PAA used as a control rather than NTC-SPION. A549 cells were plated at a density of $2 \times 10^{5}$ cells/well in DMEM $(10 \%$ FBS $)(1500 \mu \mathrm{L})$ on glass cover slips in a 6 well plate. After $24 \mathrm{~h}$, the media in the wells was replaced with fresh DMEM (1500 $\mu \mathrm{L})$ containing $0 \mu \mathrm{g} / \mathrm{ml}, 20 \mu \mathrm{g} / \mathrm{ml}$ or $200 \mu \mathrm{g} / \mathrm{ml}$ of SPION-PAA, SASO-SPION or fluoresceinlabeled SASO-SPION. Cells were incubated for 24 hours at $37^{\circ} \mathrm{C}$, washed three times 
with PBS (100 $\mu \mathrm{l} /$ wash), and stained with FM4-64FX Lipophilic Styryl Dye (Invitrogen, Carlsbad, CA) dye before fixing with 3.7\% paraformaldehyde. Fixed cells were mounted onto slides with ProLong Gold Antifade Reagent with Dapi (Invitrogen, Carlsbad, CA). Cells were viewed with Zeiss LSM 510 laser scanning confocal microscope (Zeiss). Image processing was done in Zeiss LSM Image Browser Ver. 4.2.0.121.

2.5.14 Fluorescent Microscopy. SPIONs doses in this experiment were based on iron concentration, as determined by ICP analysis, and SPION-PAA was used as a control rather than NTC-SPION. A549 cells were plated at $1 \times 10^{5}$ cells/well on glass cover slips in a 6 well plate. DMEM (10\% FBS) $(1500 \mu \mathrm{L})$ was added to each well and plates were incubated at $37^{\circ} \mathrm{C}$. After 24 hours, the media in the wells was replaced with fresh $10 \%$ FBS media containing SPION-PAA, SASO-SPION or fluorescein-labeled SASO-SPION $(0,3$, or $33 \mu \mathrm{g} \mathrm{Fe} / \mathrm{ml})$. Cells were incubated for an additional 24 hours at $37^{\circ} \mathrm{C}$. Media was removed and cells were washed three times with DPBS. Endosomes were stained with Lysotracker deep red (250 nM, 20 min, $\left.37^{\circ} \mathrm{C}\right)$ (Invitrogen, Carslbad, CA). Cells were fixed in 3.7\% paraformaldehyde and mounted with ProLong Gold Antifade Reagent with Dapi $(20 \mu \mathrm{l})$. Slides were viewed with Leica DMI 6000 inverted fluorescent microscope with a DFC300FX camera (Leica, Buffalo Grove, IL). All images were acquired using the same L5 channel settings.

\subsection{Acknowledgements}


This work was supported by the WVU Health Sciences Center. Imaging experiments and image analysis were performed in the West Virginia University Imaging Facility, which is supported in part by the Mary Babb Randolph Cancer Center and NIH grant P30 RR032138 (CoBRE III-After July 1, 2011). We acknowledge use of the WVU Shared Research Facilities. 


\subsection{Figures and Tables}

\subsubsection{Figures}

Figure 2.1. Schematic of SPION platform.
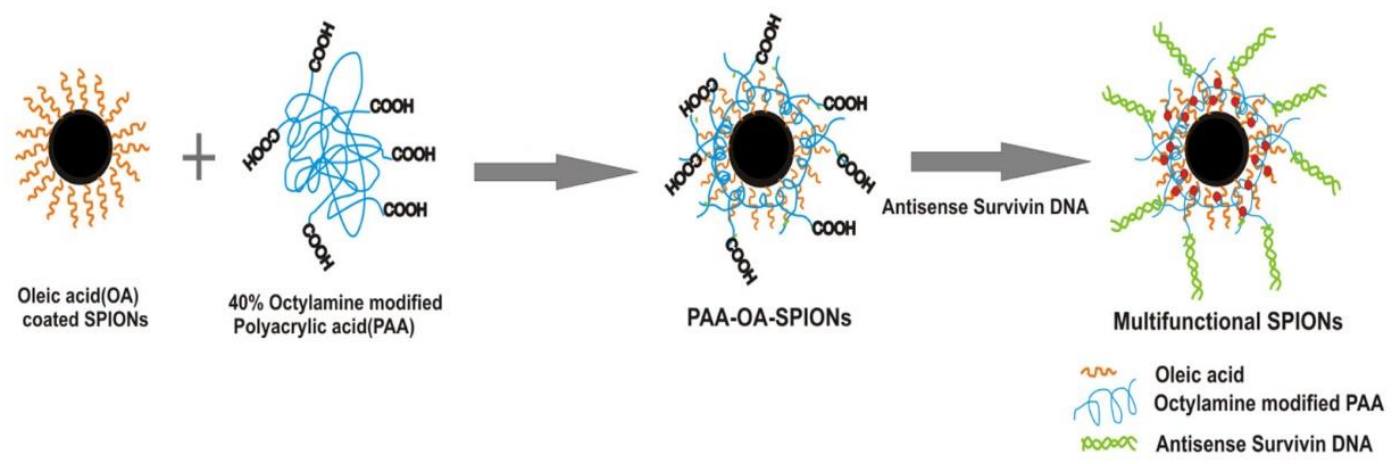

Figure 2.1. Schematic of SPION platform. (Left) SPION-OA is synthesized via thermal decomposition synthesis, (center) coated with octylamine modified PAA, and (left) aminemodified DNA is coupled to the outward-facing carboxylic acid groups of the PAA coating. The hydrophobic layer formed by alkyl tails of the oleic acid and octylamine could be loaded with lipophilic drugs (red dots). 
Figure 2.2. Diameter of SPION-OA by TEM and DLS.
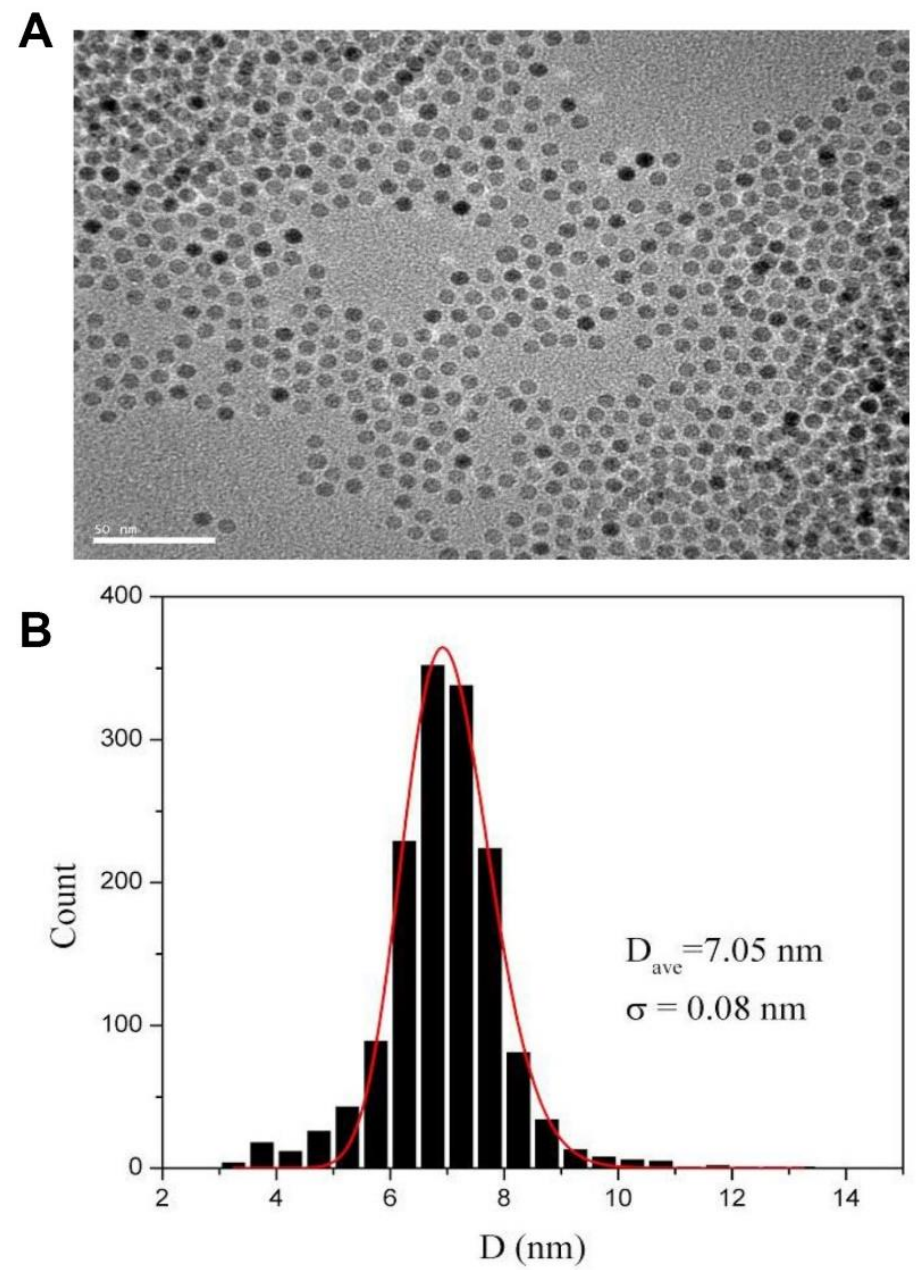

Figure 2.2. (A) Representative TEM image of SPION-OA. SPIONs are well dispersed and have uniform iron oxide cores. (B) SPION-OA iron oxide core diameter, measured across three separately prepared syntheses, is $7.05 \pm 0.08 \mathrm{~nm}$. 
Figure 2.3. FT-IR spectra of SPION-OA and SPION-PAA.
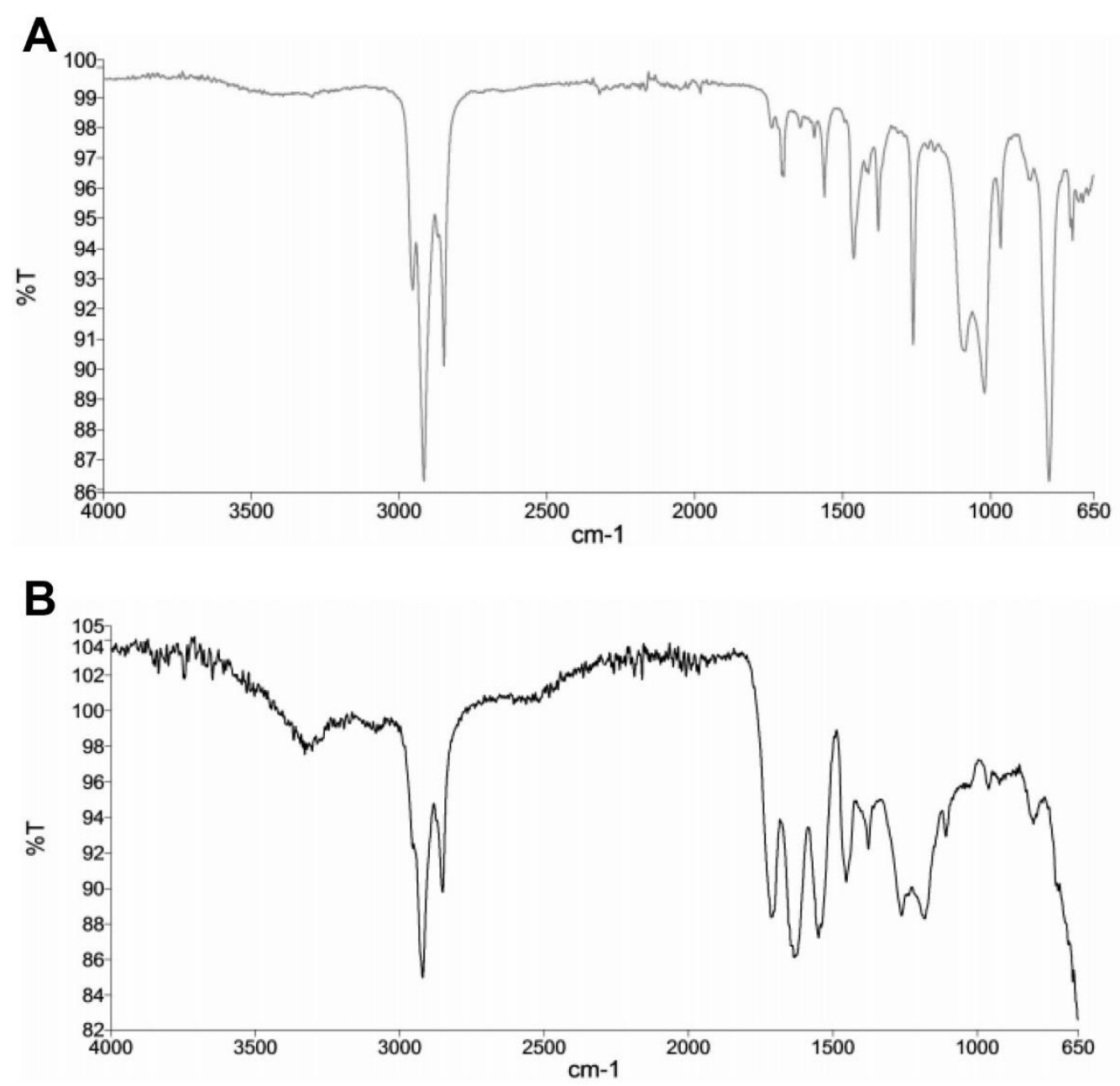

Figure 2.3. (A) FT-IR spectra for SPION-OA and (B) SPION-PAA. Analysis was performed on desiccator-dried powder samples. 
Figure 2..4 TGA of SPION-OA and SPION-PAA.
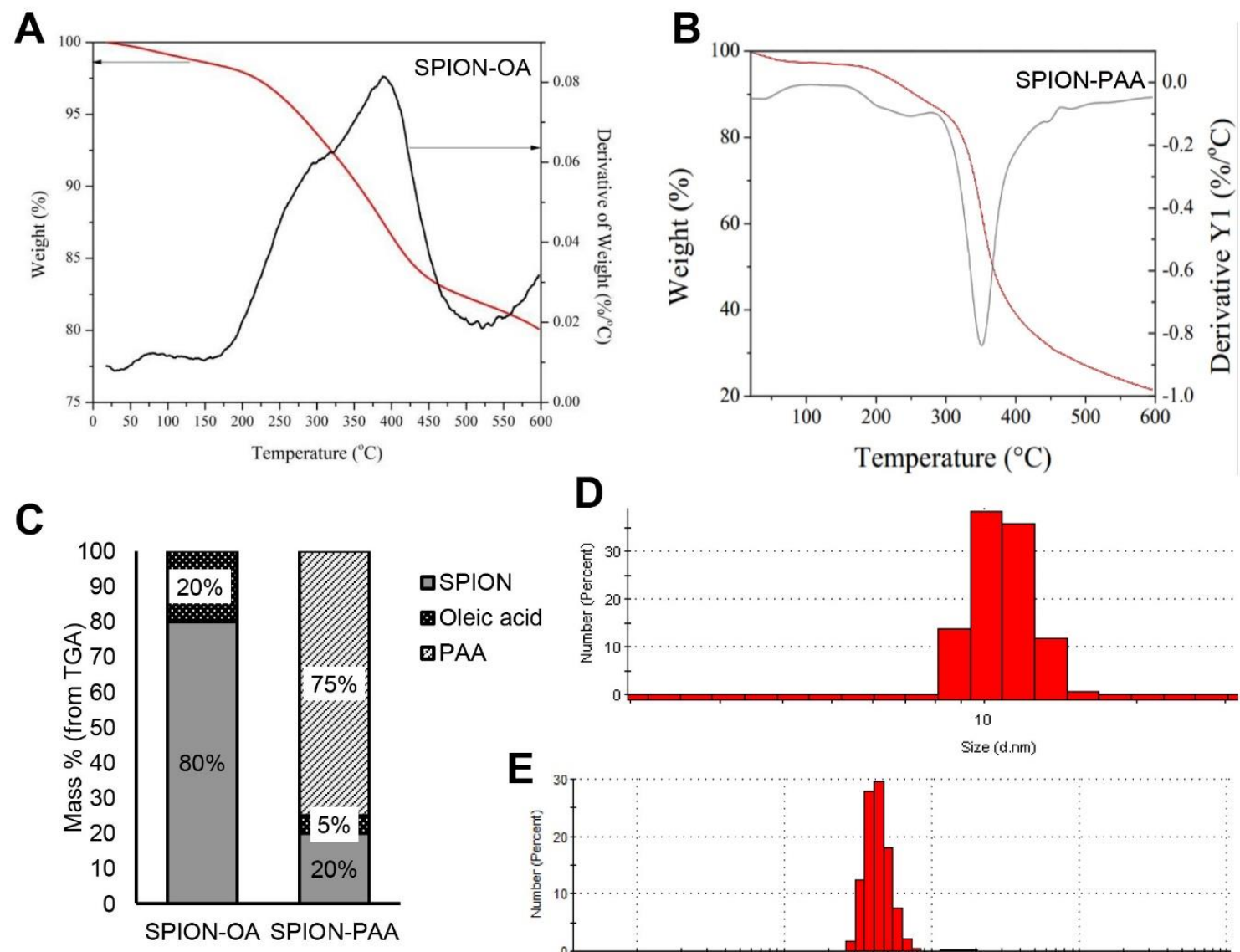

D
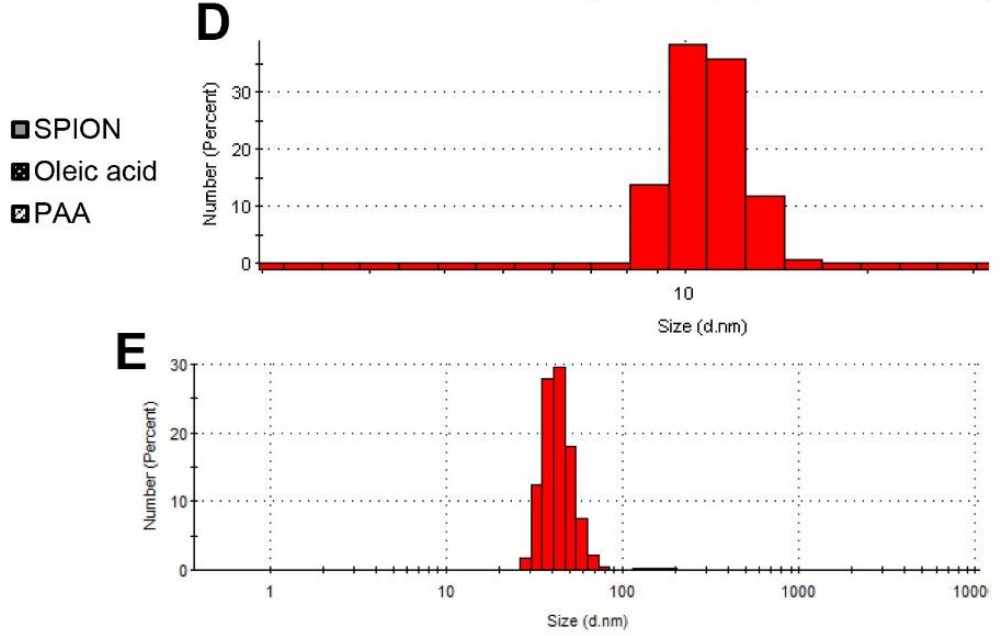

Figure 2.4. A) Representative TGA of SPION-OA and (B) SPION-PAA. Red line shows percent of original mass remaining as temperature was increased $\left(5^{\circ} \mathrm{C} /\right.$ minute $)$. Black line is the derivative of the red line, showing the rate of change of the mass as temperature was increased. (C) Graphical representation of the composition of SPION-OA and SPION-PAA. Composition was determined by TGA and was based on the analysis of three samples from three separate batches. (D) Hydrodynamic diameter of SPION-OA in toluene and (E) SPION-PAA in distilled water. 
Figure 2.4. Diameter of SASO-SPION by TEM and DLS.
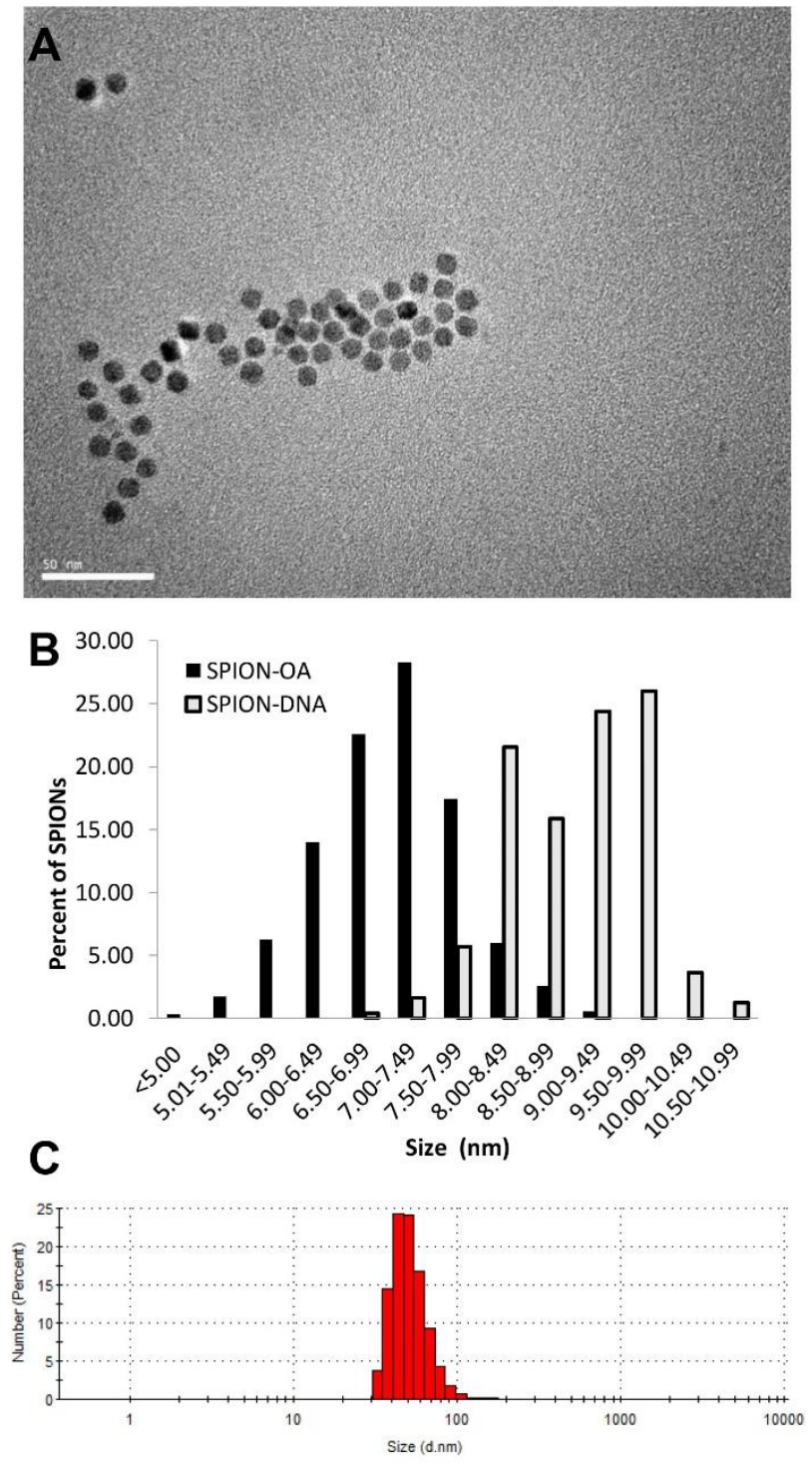

Figure 2.5. (A) Representative TEM image of SASO-SPION. SASO-SPION is well dispersed and has uniform iron oxide cores. (B) Graphical comparison of iron oxide core diameter of a single batch of SPION-OA $(7.07 \pm 0.74 \mathrm{~nm})$ and SASO-SPION $(7.07 \pm 0.74$ $\mathrm{nm}$ ) prepared from that batch. (C) Hydrodynamic diameter of SASO-SPION in distilled water, as determined by DLS, is $50.58 \mathrm{~nm}$. 
Figure 2.6. To-scale diagram of contribution of SPION layers to final particle diameter.

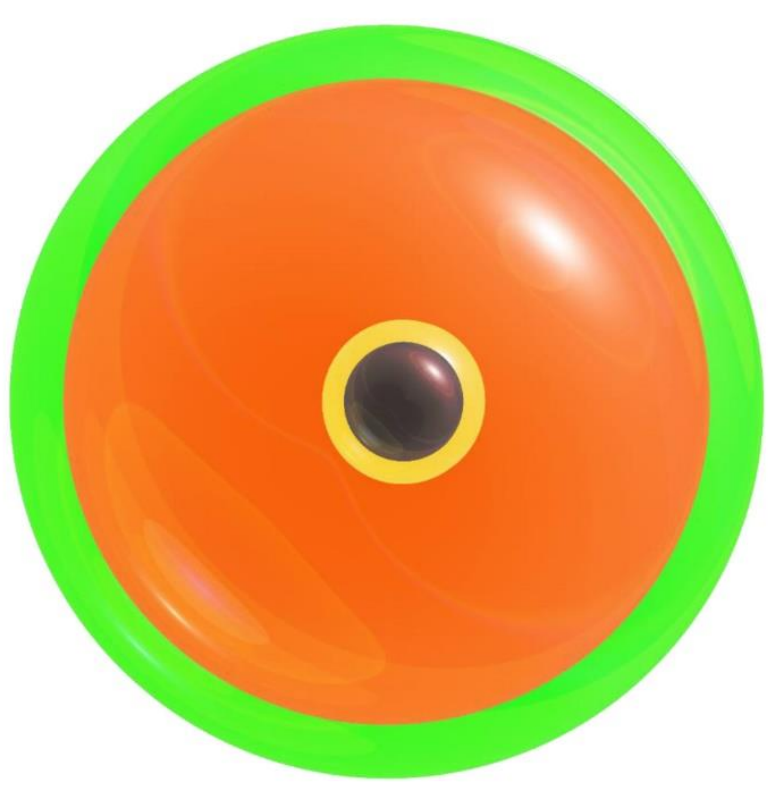

Figure 2.6. To-scale diagram of contribution of various SPION layers to hydrodynamic diameter (determined by DLS). 
Figure 2.. SASO-SPION is internalized by A549 cells.

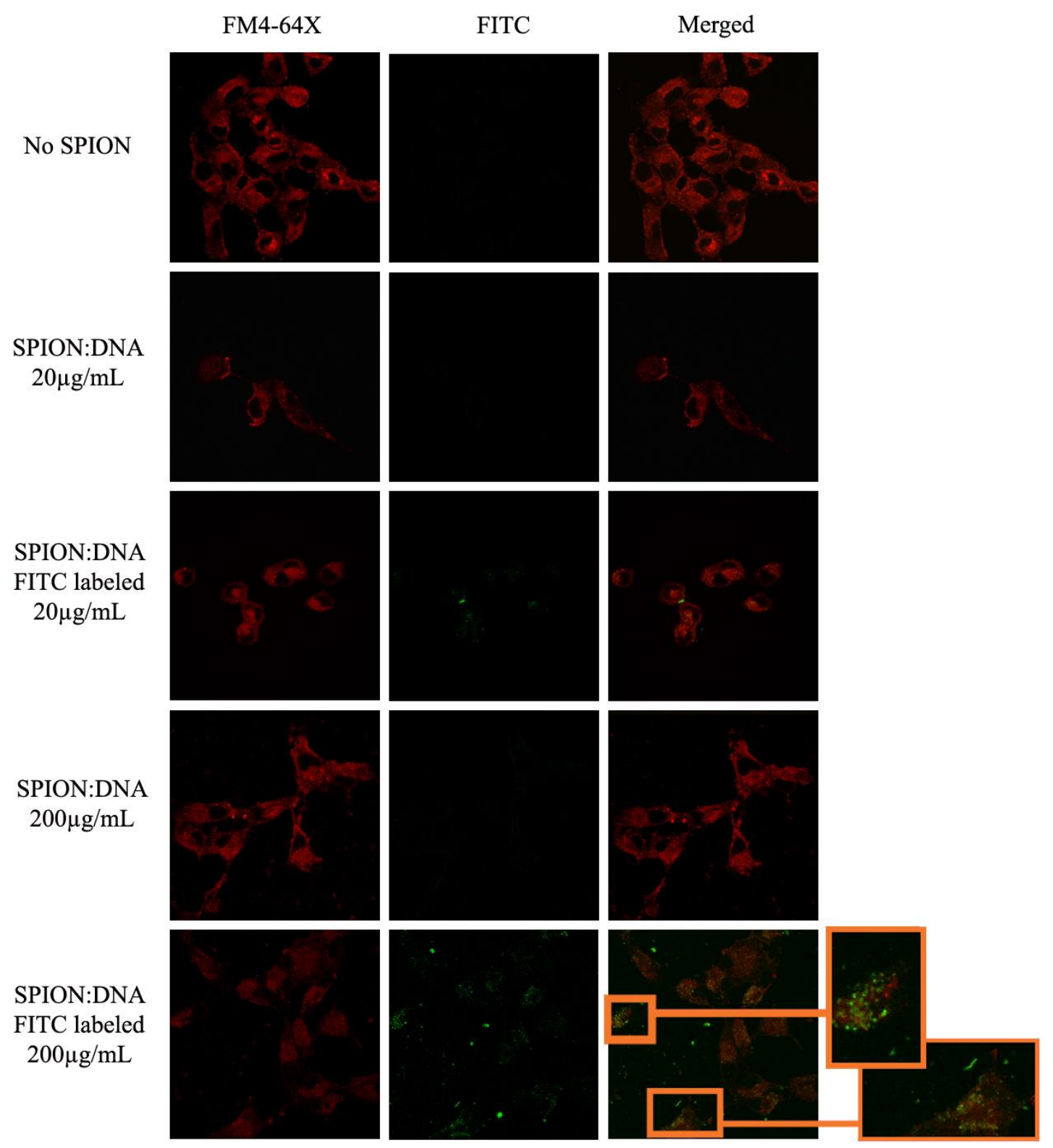

Figure 2.7. SASO-SPION is taken up by A549 cells following 24 hour incubation. Cells were incubated with SASO-SPION or fluorescein- SASO-SPION $(0,20,200 \mu \mathrm{g} / \mathrm{mL})$ for 24 hours, washed to remove free SPIONs, and stained with FM4-64X cell membrane stain before fixation. Fluorescein- SASO-SPION is visible inside the cells, particularly at the high $(200 \mu \mathrm{g} / \mathrm{mL})$ dose, as shown in the insets. 
Figure 2,8 SASO-SPION is detectable in endosomes of A549 cells after 24 hour exposure.
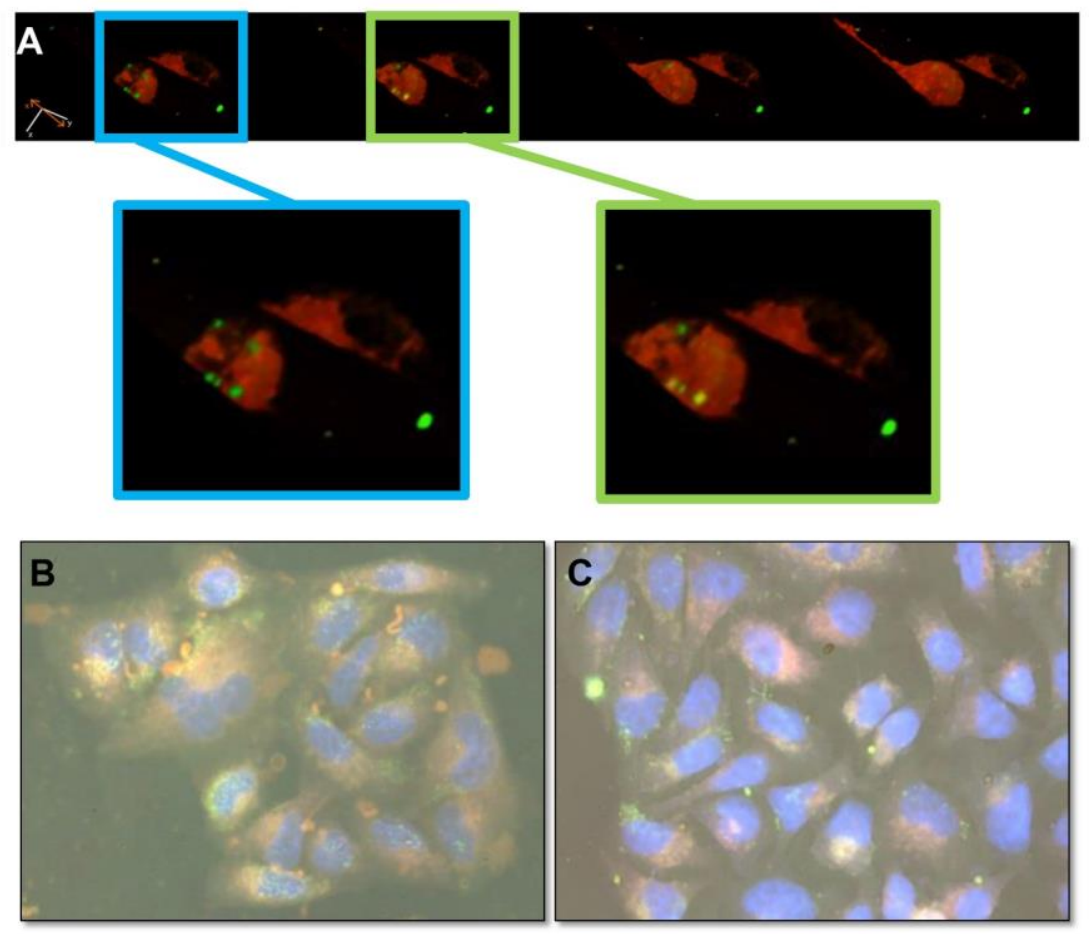

Figure 2.8. (A) Fluorescently-labeled SASO-SPION is taken up by A549 cells following a 24 hour incubation. Cells were incubated with SASO-SPION or fluorescein-SASO-SPION $(200 \mu \mathrm{g} / \mathrm{mL})$ for 24 hours, washed to remove free SPIONs, and stained with FM4-64X cell membrane stain before fixation.3D reconstruction from confocal microscopy z-stack images shows that fluorescein-SASO-SPIONs (green) are inside of the FM4-64X stained cells (red). (B) Fluorescent microscopy shows co-localization (yellow) of fluoresceintagged SASO-SPION (green) and endosomes (red) after staining with Lysotracker Deep Red (left) or Cell Mask Orange (right). Cells were incubated with fluorescein-labeled SASO-SPION (33 $\mu \mathrm{g} \mathrm{Fe} / \mathrm{mL}$ ) for 24 hours, washed to remove free SPIONs, and stained with either Lysotracker Deep Red or Cell Mask Orange cell membrane stain before fixation. 
Figure 2.9. SASO-SPION decreases survivin expression in A549 cells.
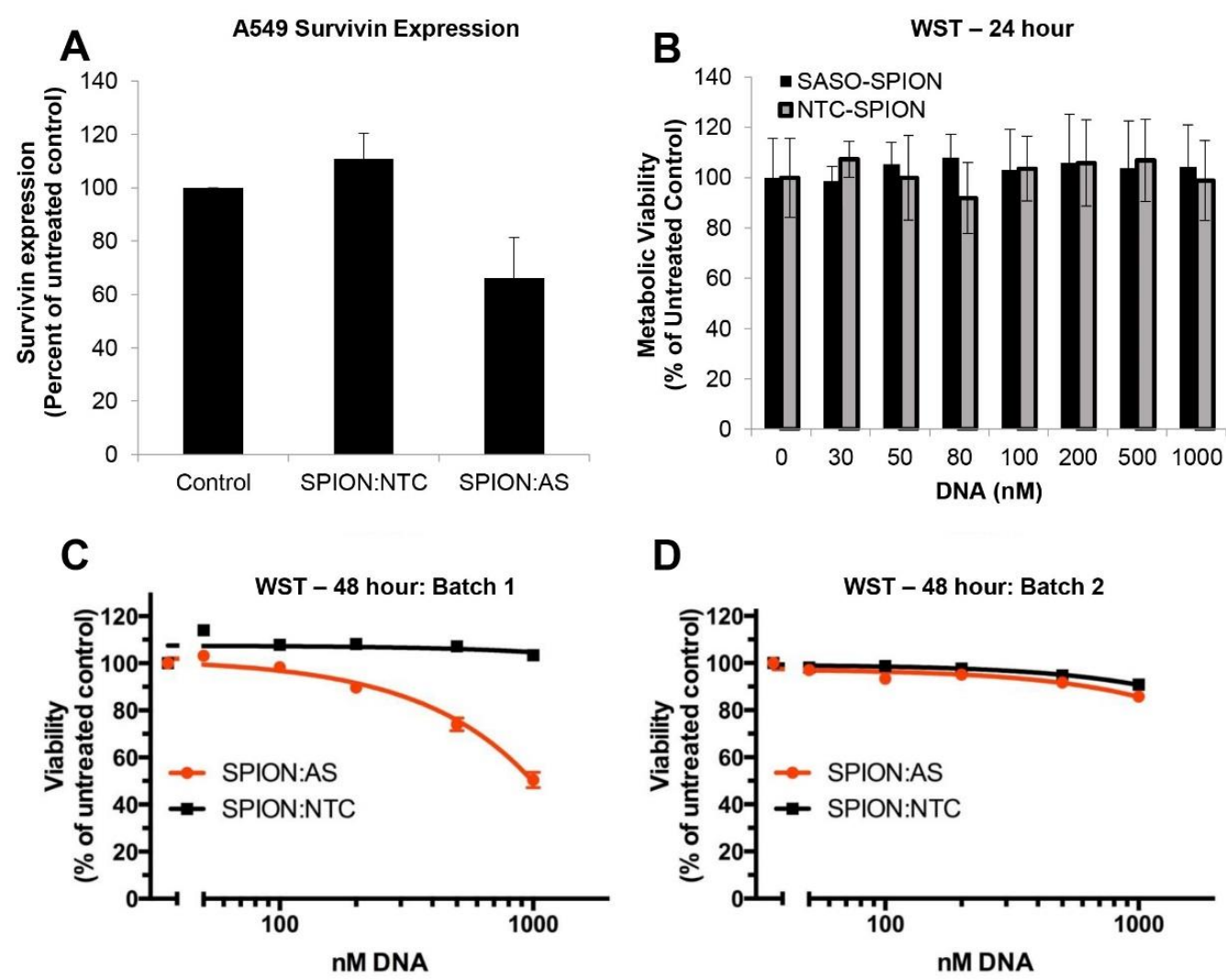

Figure 2.9. (A) Survivin expression in A549 cells following 24 hour incubation. Results from 2 trials. (B) Metabolic viability, determined by WST-1 assay, of A549 cells was assessed after 24 hour incubation with SASO-SPION or NTC-SPION $(0,50,100,200$, $500,1000 \mathrm{nM}$ DNA). Results are expressed as a percentage of the untreated control viability. Results of 4 independent trials. No significance $(p<0.05$, by Student's T-test) was noted. (C, D) Metabolic viability, determined by WST-1 assay, of A549 cells was assessed after 48 hour incubation with SASO-SPION or NTC-SPION $(0,50,100,200$, 500, $1000 \mathrm{nM}$ DNA). Results are expressed as a percentage of the untreated control viability. (C) and (D) show the variation in biological effects resulting from inconsistencies in the DNA coupling process, despite treating cells with the same DNA concentration. 


\subsubsection{Tables}

Table 2.1. Contribution of various SPION layers to total particle diameter.

\begin{tabular}{|c|c|c|c|c|}
\hline & SPION & SPION-OA & SPION-PAA & SPION:DNA \\
\hline $\begin{array}{l}\text { Diameter } \\
\text { (DLS) }\end{array}$ & $7.00 \mathrm{~nm}$ & $11.27 \mathrm{~nm}$ & $43.12 \mathrm{~nm}$ & $50.28 \mathrm{~nm}$ \\
\hline $\begin{array}{l}\text { Change from } \\
\text { previous } \\
\text { step }\end{array}$ & ------ & $4.27 \mathrm{~nm}$ & $32.05 \mathrm{~nm}$ & $7.46 \mathrm{~nm}$ \\
\hline $\begin{array}{l}\text { Length of } 1 \\
\text { addition }\end{array}$ & ------- & OA: $1.97 \mathrm{~nm}$ & $? ?$ & $\begin{array}{l}\text { DNA: } 3.2 \mathrm{~nm} \\
\mathbf{C}_{6} \mathbf{N H}: 0.5 \mathrm{~nm}\end{array}$ \\
\hline $\begin{array}{c}\text { Expected } \\
\text { change }\end{array}$ & ------- & $4 \mathrm{~nm}$ & $? ?$ & $7.4 \mathrm{~nm}$ \\
\hline
\end{tabular}

Table 2.1. Contribution of various SPION layers to total particle diameter. Particle diameters were determined using DLS. Expected changes were calculated based on a single layer of covalently bound coating for both oleic acid and DNA. 


\subsection{References}

1. Olie, R. A., Simões-wüst, A. P., Baumann, B. \& Simo, A. P. A Novel Antisense Oligonucleotide Targeting Survivin Expression Induces Apoptosis and Sensitizes Lung Cancer Cells to Chemotherapy. Cancer Res. 60, 2805-2809 (2000).

2. Ambrosini, G., Adida, C. \& Altieri, D. C. A novel anti-apoptosis gene, survivin, expressed in cancer and lymphoma. Nat. Med. 3, 917-921 (1997).

3. Altieri, D. C. Validating survivin as a cancer therapeutic target. Nat. Rev. Cancer 3, 46-54 (2003).

4. Hirano, H. et al. Survivin expression in lung cancer: Association with smoking, histological types and pathological stages. Oncol. Lett. 10, 1456-1462 (2015).

5. Xia, C. et al. Induction of Apoptosis in Mesothelioma Cells by Antisurvivin Oligonucleotides. Mol. Cancer Ther. 1, 687-694 (2002).

6. Schlette, E. J., Medeiros, L. J., Goy, A., Lai, R. \& Rassidakis, G. Z. Survivin Expression Predicts Poorer Prognosis in Anaplastic Large-Cell Lymphoma. J. Clin. Oncol. 22, 1682-8 (2004).

7. Trieb, K., Lehner, R., Stulnig, T., Sulzbacher, I. \& Shroyer, K. R. Survivin expression in human osteosarcoma is a marker for survival. Eur. J. Surg. Oncol. 29, 379-382 (2003).

8. Das, A., Tan, W.-L., Teo, J. \& Smith, D. R. Expression of survivin in primary glioblastomas. J. Cancer Res. Clin. Oncol. 128, 302-6 (2002). 
9. Zhang, M., Latham, D. E., Delaney, M. a \& Chakravarti, A. Survivin mediates resistance to antiandrogen therapy in prostate cancer. Oncogene 24, 2474-82 (2005).

10. Ai, Z. et al. Inhibition of survivin reduces cell proliferation and induces apoptosis in human endometrial cancer. Cancer 107, 746-56 (2006).

11. Ferrandina, G. et al. Survivin expression in ovarian cancer and its correlation with clinico-pathological, surgical and apoptosis-related parameters. Br. J. Cancer 92, $271-7$ (2005).

12. Kami, K. et al. Survivin expression is a prognostic marker in pancreatic cancer patients. Surgery 136, 443-8 (2004).

13. Altieri, D. C. The molecular basis and potential role of survivin in cancer diagnosis and therapy. Trends Mol. Med. 7, 542-7 (2001).

14. Kawasaki, H., Altieri, D. C. \& Lu, C. Inhibition of Apoptosis by Survivin Predicts Shorter Survival Rates in Colorectal Cancer. Cancer Res. 58, 5071-5074 (1998).

15. Li, H., Niederkorn, J. Y., Neelam, S. \& Alizadeh, H. Downregulation of survivin expression enhances sensitivity of cultured uveal melanoma cells to cisplatin treatment. Exp. Eye Res. 83, 176-82 (2006).

16. Osaka, E. et al. Survivin as a prognostic factor for osteosarcoma patients. Acta Histochem. Cytochem. 39, 95-100 (2006). 
17. Carrasco, R. a et al. Antisense inhibition of survivin expression as a cancer therapeutic. Mol. Cancer Ther. 10, 221-32 (2011).

18. Chen, J. et al. Down-regulation of survivin by antisense oligonucleotides increases apoptosis, inhibits cytokinesis and anchorage-independent growth. Neoplasia 2, 235-41 (2000).

19. Cao, C., Mu, Y., Hallahan, D. E. \& Lu, B. XIAP and survivin as therapeutic targets for radiation sensitization in preclinical models of lung cancer. Oncogene 23, 704752 (2004).

20. Fuessel, S. et al. Systematic in Vitro Evaluation of Survivin Directed Antisense Oligodeoxynucleotides in Bladder Cancer Cells. J. Urol. 171, 2471-2476 (2004).

21. Kappler, M. et al. Knockdown of survivin expression by small interfering RNA reduces the clonogenic survival of human sarcoma cell lines independently of p53. Cancer Gene Ther. 11, 186-93 (2004).

22. Lu, B. et al. Survivin As a Therapeutic Target for Radiation Sensitization in Lung Cancer Survivin As a Therapeutic Target for Radiation Sensitization in Lung Cancer. Cancer Res 64, 2840-2845 (2004).

23. Giaccone, G. et al. Multicenter phase II trial of YM155, a small-molecule suppressor of survivin, in patients with advanced, refractory, non-small-cell lung cancer. J. Clin. Oncol. 27, 4481-6 (2009). 
24. Wobser, M. et al. Complete remission of liver metastasis of pancreatic cancer under vaccination with a HLA-A2 restricted peptide derived from the universal tumor antigen survivin. Cancer Immunol. Immunother. 55, 1294-8 (2006).

25. Talbot, D. C. et al. Tumor survivin is downregulated by the antisense oligonucleotide LY2181308: a proof-of-concept, first-in-human dose study. Clin. Cancer Res. 16, 6150-8 (2010).

26. De Mesmaeker, A., Haener, R., Martin, P. \& Moser, H. E. Antisense Oligonucleotides. Acc. Chem. Res. 28, 366-374 (1995).

27. Dominska, M. \& Dykxhoorn, D. M. Breaking down the barriers: siRNA delivery and endosome escape. J. Cell Sci. 123, 1183-9 (2010).

28. Dias, N. \& Stein, C. A. Antisense Oligonucleotides: Basic Concepts and Mechanisms. Mol. Cancer Ther. 1, 347-355 (2002).

29. Chu, T. C., Twu, K. Y., Ellington, A. D. \& Levy, M. Aptamer mediated siRNA delivery. Nucleic Acids Res. 34, e73 (2006).

30. Jepsen, J. S., Sorensen, M. D. \& Wengel, J. Locked Nucleic Acid: A Potent Nucleic Acid Analog in herapeutics and Biotechnology. Oligonucleotides 14, 130-146 (2004).

31. Boirivant, M. et al. Inhibition of Smad7 with a specific antisense oligonucleotide facilitates TGF-beta1-mediated suppression of colitis. Gastroenterology 131, 178698 (2006). 
32. Monteleone, G. et al. Mongersen, an oral SMAD7 antisense oligonucleotide, and Crohn's disease. N. Engl. J. Med. 372, 1104-13 (2015).

33. Shim, M. S. \& Kwon, Y. J. Efficient and targeted delivery of siRNA in vivo. FEBS J. 277, 4814-27 (2010).

34. Rosen, J. E., Chan, L., Shieh, D.-B. \& Gu, F. X. Iron oxide nanoparticles for targeted cancer imaging and diagnostics. Nanomedicine 8, 275-90 (2012).

35. Wahajuddin \& Arora, S. Superparamagnetic iron oxide nanoparticles: magnetic nanoplatforms as drug carriers. Int. J. Nanomedicine 7, 3445-71 (2012).

36. Burtea, C. et al. In vitro biomedical applications of functionalized iron oxide nanoparticles, including those not related to magnetic properties. Contrast Media Mol. Imaging 6, 236-50 (2011).

37. Jin, R., Lin, B., Li, D. \& Ai, H. Superparamagnetic iron oxide nanoparticles for MR imaging and therapy: design considerations and clinical applications. Curr. Opin. Pharmacol. 18, 18-27 (2014).

38. Laurent, S. \& Mahmoudi, M. Superparamagnetic iron oxide nanoparticles: promises for diagnosis and treatment of cancer. Int. J. Mol. Epidemiol. Genet. 2, 367-90 (2011).

39. Mahajan, S., Koul, V., Choudhary, V., Shishodia, G. \& Bharti, A. C. Preparation and in vitro evaluation of folate-receptor-targeted SPION-polymer micelle hybrids for MRI contrast enhancement in cancer imaging. Nanotechnology 24, 015603 (2013). 
40. Ai, J. et al. Nanotoxicology and nanoparticle safety in biomedical designs. Int. J. Nanomedicine 6, 1117-27 (2011).

41. Li, S.-D. \& Huang, L. Targeted delivery of antisense oligodeoxynucleotide and small interference RNA into lung cancer cells. Mol. Pharm. 3, 579-88 (2006).

42. Kaaki, K. et al. Magnetic nanocarriers of doxorubicin coated with poly(ethylene glycol) and folic acid: relation between coating structure, surface properties, colloidal stability, and cancer cell targeting. Langmuir 28, 1496-505 (2012).

43. Omidirad, R., Rajabi Hosseinpour, F. \& Farahani, B. Preparation and in vitro drug delivery response of doxorubicin loaded PAA coated magnetite nanoparticles. J. Serbian Chem. Soc. 78, 1609-1616 (2013).

44. Hyeon, T., Lee, S. S., Park, J., Chung, Y. \& Na, H. B. Synthesis of highly crystalline and monodisperse maghemite nanocrystallites without a size-selection process. J. Am. Chem. Soc. 123, 12798-801 (2001).

45. Shen, H., Jawaid, A. M. \& Snee, P. T. Poly(ethylene glycol) carbodiimide coupling reagents for the biological and chemical functionalization of water-soluble nanoparticles. ACS Nano 3, 915-23 (2009).

46. Wagner, K. et al. Synthesis of oligonucleotide-functionalized magnetic nanoparticles and study on their in vitro cell uptake. Appl. Organomet. Chem. 18, $514-519$ (2004). 
47. Tamm, I. et al. IAP-Family Protein Survivin Inhibits Caspase Activity and Apoptosis Induced by Fas (CD95), Bax, Caspases, and Anticancer Drugs. Cancer Res. 58, $5315-5320$ (1998).

48. Pisane, K., Despeaux, E. \& Seehra, M. S. Magnetic relaxation and correlating effective magnetic moment with particle size distribution in maghemite nanoparticles. J. Magn. Magn. Mater.

49. Yu, W. W., Chang, E., Drezek, R. \& Colvin, V. L. Water-soluble quantum dots for biomedical applications. Biochem. Biophys. Res. Commun. 348, 781-6 (2006).

50. Booth, M. A., Kannappan, K., Hosseini, A. \& Partridge, A. In-Depth Electrochemical Investigation of Surface Attachment Chemistry via Carbodiimide Coupling. Langmuir 31, 8033-41 (2015).

51. Liu, L. et al. Activity analysis of the carbodiimide-mediated amine coupling reaction on self-assembled monolayers by cyclic voltammetry. Electrochim. Acta 89, 616$622(2013)$

52. Wrobel, N., Schinkinger, M. \& Mirsky, V. M. A novel ultraviolet assay for testing side reactions of carbodiimides. Anal. Biochem. 305, 135-8 (2002).

53. Conde, J. et al. Revisiting 30 years of biofunctionalization and surface chemistry of inorganic nanoparticles for nanomedicine. Front. Chem. 2, 48 (2014). 
54. Smith, S. M., Wunder, M. B., Norris, D. a \& Shellman, Y. G. A simple protocol for using a LDH-based cytotoxicity assay to assess the effects of death and growth inhibition at the same time. PLoS One 6, e26908 (2011).

55. Mahmoudi, M., Sahraian, M. a, Shokrgozar, M. a \& Laurent, S. Superparamagnetic iron oxide nanoparticles: promises for diagnosis and treatment of multiple sclerosis. ACS Chem. Neurosci. 2, 118-40 (2011).

56. Petri-Fink, A., Steitz, B., Finka, A., Salaklang, J. \& Hofmann, H. Effect of cell media on polymer coated superparamagnetic iron oxide nanoparticles (SPIONs): colloidal stability, cytotoxicity, and cellular uptake studies. Eur. J. Pharm. Biopharm. 68, 129-37 (2008).

57. Nel, A. E. et al. Understanding biophysicochemical interactions at the nano-bio interface. Nat. Mater. 8, 543-57 (2009).

58. Boutry, S. et al. Magnetic labeling of non-phagocytic adherent cells with iron oxide nanoparticles: a comprehensive study. Contrast Media Mol. Imaging 3, 223-32 (2008).

59. Aggarwal, P., Hall, J. B., McLeland, C. B., Dobrovolskaia, M. a \& McNeil, S. E. Nanoparticle interaction with plasma proteins as it relates to particle biodistribution, biocompatibility and therapeutic efficacy. Adv. Drug Deliv. Rev. 61, 428-37 (2009).

60. Neuberger, T., Schöpf, B., Hofmann, H., Hofmann, M. \& von Rechenberg, B. Superparamagnetic nanoparticles for biomedical applications: Possibilities and 
limitations of a new drug delivery system. J. Magn. Magn. Mater. 293, 483-496 (2005).

61. Smith, A. M. \& Nie, S. Minimizing the hydrodynamic size of quantum dots with multifunctional multidentate polymer ligands. J. Am. Chem. Soc. 130, 11278-9 (2008).

62. Sato, K., Tachibana, Y., Hattori, S., Chiba, T. \& Kuwabata, S. Polyacrylic acid coating of highly luminescent CdS nanocrystals for biological labeling applications. J. Colloid Interface Sci. 324, 257-60 (2008).

63. Carmen Bautista, M., Bomati-Miguel, O., del Puerto Morales, M., Serna, C. J. \& Veintemillas-Verdaguer, S. Surface characterisation of dextran-coated iron oxide nanoparticles prepared by laser pyrolysis and coprecipitation. J. Magn. Magn. Mater. 293, 20-27 (2005).

64. Thorek, D. L. J., Elias, D. R. \& Tsourkas, A. Comparative Analysis of NanoparticleAntibody Conjugations: Carbodiimide versus Click Chemistry. Mol. Imaging 8, 221229 (2009).

65. Thompson, K. \& Michielsen, S. Novel synthesis of N-substituted polyacrylamides: Derivatization of poly(acrylic acid) with amines using a triazine-based condensing reagent. J. Polym. Sci. Part A Polym. Chem. 44, 126-136 (2006).

66. Palazon, F. et al. Carbodiimide/NHS Derivatization of $\mathrm{COOH}-$ Terminated SAMs: Activation or Byproduct Formation? Langmuir 30, 4545-4550 (2014). 
67. Sam, S. et al. Semiquantitative study of the EDC/NHS activation of acid terminal groups at modified porous silicon surfaces. Langmuir 26, 809-14 (2010).

68. Wang, T.-H. \& Lee, W.-C. Immobilization of proteins on magnetic nanoparticles. Biotechnol. Bioprocess Eng. 8, 263-267 (2003).

69. Walsh, M. K., Wang, X. \& Weimer, B. C. Optimizing the immobilization of singlestranded DNA onto glass beads. J. Biochem. Biophys. Methods 47, 221-231 (2001).

70. Christiaens, P. et al. EDC-mediated DNA attachment to nanocrystalline CVD diamond films. Biosens. Bioelectron. 22, 170-177 (2006).

71. Millan, K. M., Saraullo, a \& Mikkelsen, S. R. Voltammetric DNA biosensor for cystic fibrosis based on a modified carbon paste electrode. Anal. Chem. 66, 2943-2948 (1994).

72. Shen, H., Jawaid, A. M. \& Snee, P. T. Poly (ethylene glycol) Carbodiimide Coupling Reagents for the Biological and Chemical Functionalization of Water- Soluble Nanoparticles. ACS Nano 3, 915-23 (2009).

73. Kim, J.-S. et al. Cellular uptake of magnetic nanoparticle is mediated through energy-dependent endocytosis in A549 cells. J. Vet. Sci. 7, 321-326 (2006).

74. Rosi, N. L. et al. Oligonucleotide-modified gold nanoparticles for intracellular gene regulation. Science (80-. ). 312, 1027-30 (2006). 
75. Patel, P. C. et al. Scavenger Receptors Mediate Cellular Uptake of Polyvalent Oligonucleotide-Functionalized Gold Nanoparticles. Bioconjug. Chem. 21, 22502256 (2010).

76. Cutler, J. I., Auyeung, E. \& Mirkin, C. a. Spherical nucleic acids. J. Am. Chem. Soc. 134, 1376-91 (2012).

77. Lee, G. Y. et al. Theranostic Nanoparticles with Controlled Release of Gemcitabine for Targeted Therapy and MRI of Pancreatic Cancer. ACS Nano 7, 2078-2089 (2013).

78. Zhu, L. et al. Multifunctional pH-sensitive superparamagnetic iron-oxide nanocomposites for targeted drug delivery and MR imaging. J. Control. Release $169,228-38$ (2013).

79. Seth, S. et al. RNAi-based therapeutics targeting survivin and PLK1 for treatment of bladder cancer. Mol. Ther. 19, 928-35 (2011).

80. Natarajan, A. et al. NanoFerrite Particle Based Radioimmunonanoparticles: Binding affinity and in vivo pharmacokinetics. Bioconjug. Chem. 19, 1211-1218 (2008).

81. Kocbek, P., Obermajer, N., Cegnar, M., Kos, J. \& Kristl, J. Targeting cancer cells using PLGA nanoparticles surface modified with monoclonal antibody. J. Control. release 120, 18-26 (2007). 
82. Lin, C.-C. et al. A KLF4-miRNA-206 autoregulatory feedback loop can promote or inhibit protein translation depending upon cell context. Mol. Cell. Biol. 31, 2513-27 (2011).

83. Kroll, A., Pillukat, M. H., Hahn, D. \& Schnekenburger, J. Current in vitro methods in nanoparticle risk assessment: limitations and challenges. Eur. J. Pharm. Biopharm. 72, 370-7 (2009).

84. Mahmoudi, M., Hofmann, H., Rothen-Rutishauser, B. \& Petri-Fink, A. Assessing the in vitro and in vivo toxicity of superparamagnetic iron oxide nanoparticles. Chem. Rev. 112, 2323-38 (2012).

85. Bonakdar, S. \& Mashinchian, O. in Stem-Cell Eng. (Baharvand, H. \& Aghdami, N.) 171-184 (John Wiley \& Sons, Inc., 2015). 


\section{Chapter 3}

\section{Role of Mesothelin in Carbon Nanotube-Induced Carcinogenic Transformation of Human Bronchial Epithelial Cells}

*American Journal of Physiology: Lung Cellular and Molecular Biology

Xiaoqing He ${ }^{1 \dagger}$, ${ }^{*} E m i l y ~ D e s p e a u x^{1 \dagger}$, Todd A. Stueckle ${ }^{1,2}$, Alexander Chi ${ }^{3}$, Vincent Castranova ${ }^{{ }^{*}}$, Cerasela Zoica Dinu ${ }^{4}$, Liying Wang ${ }^{2}$, and Yon Rojanasakul. ${ }^{1,3}{ }^{1}$ Department of Pharmaceutical Sciences, ${ }^{4}$ Department of Chemical Engineering, and ${ }^{3}$ WVU Cancer Institute, West Virginia University, Morgantown, WV 26506; ${ }^{2}$ HELD, National Institute for Occupational Safety and Health, Morgantown, WV 26505. 


\subsection{Abstract}

Carbon nanotubes (CNTs) have been likened to asbestos in terms of morphology and toxicity. CNT exposure can lead to pulmonary fibrosis and promotion of tumorigenesis. However, the mechanisms underlying CNT-induced carcinogenesis are not well defined. Mesothelin (MSLN) is over-expressed in many human tumors, including mesotheliomas and pancreatic and ovarian carcinomas. In this study, the role of MSLN in the carcinogenic transformation of human bronchial epithelial cells chronically exposed to single-walled CNT (BSW) was investigated. MSLN overexpression was found in human lung tumors, lung cancer cell lines, and BSW cells. The functional role of MSLN in the BSW cells was then investigated using stably-transfected MSLN knockdown (BSW shMSLN) cells. MSLN knockdown resulted in significantly decreased invasion, migration, colonies on soft agar, and tumor sphere formation. In vivo, BSW shMSLN cells formed smaller primary tumors and less metastases. The mechanism by which MSLN contributes to these more aggressive behaviors was investigated using Ingenuity Pathway Analysis, which predicted that increased MSLN could induce cyclin E expression. We found that BSW shMSLN cells had decreased cyclin E, and their proliferation rate was reverted to nearly that of untransformed cells. Cell cycle analysis showed that the BSW shMSLN cells had an increased G2 population and a decreased S phase population, which is consistent with the decreased rate of proliferation. Together, our results indicate a novel role of MSLN in the malignant transformation of bronchial epithelial cells following CNT exposure, suggesting its utility as a potential biomarker and drug target for CNT-induced malignancies. 


\subsection{Introduction}

Carbon nanotubes (CNTs) have unique physical and chemical properties that make them suitable for a variety of consumer and biomedical applications. Their electrical conductivity and strength are exploited with their integration into consumer products, including electronics and sports equipment. The ease of functionalization make CNTs ideal candidates for biosensors and drug-delivery platforms $(10,32,38)$. CNTs are fibrous in shape and resistant to chemical and high-temperature degradation. Therefore, CNTs exhibit asbestos-like properties (44). Due to the rapid growth of production, worker exposure to CNTs is increasingly likely, creating a potential human health hazard (10, 42).

Pulmonary toxicity following CNT exposure is of particular concern, as CNTs are small, low density, and easily aerosolized (32). As with other nanoparticles, the toxicity of CNTs depends heavily on the dose, exposure route, and particle physical properties (38, 42). Variations in these parameters often make it difficult to compare published outcomes. However, the consensus is that inhalation of CNTs are likely detrimental to human health, but the full scope and mechanisms of the damage are still unclear (11).

Much of what is known about CNT toxicity comes from acute, high-dose exposures, which result in transient inflammation and fibrosis $(7,42)$. In reality, chronic, low-dose exposures are more likely (42), meaning that chronic exposure models are needed to determine meaningful occupational exposure limits. Additionally, CNTs are not degraded in vivo and their persistent presence, even at very low doses, can cause oxidative stress (42) and DNA damage (7). Over time, this damage could contribute to the development 
of cancer. However, the molecular mechanisms underlying this transformation are currently unknown.

To better understand the mechanism of CNT-induced carcinogenesis, we previously generated an in vitro chronic CNT exposure model by culturing human bronchial epithelial (BEAS-2B) cells with single-wall carbon nanotubes (SWCNTs; $0.02 \mu \mathrm{g} / \mathrm{cm}^{2}$ ) for 6 months $(21,47)$. These chronic SWCNT-exposed (BSW) cells had greater malignant morphology and behavior than their passage-matched parental cells, including increased proliferation rates, increased resistance to apoptosis $(30,47)$, and increased tumor formation in vivo $(21,47)$.

The malignant phenotype noted after chronic exposure to sub-acute doses of SWCNTs results from the interaction of many factors, including changes in regulation of a variety of cell processes (apoptosis, invasion/attachment, cell cycle regulation, etc.). The mechanisms causing these changes have not been fully described, but, given the similarities between CNTs and asbestos, it is possible that the pathologies could develop through similar mechanisms.

Mesothelin (MSLN), a $40 \mathrm{kD}$ cell-surface protein of unknown physiological significance, is found in very few normal tissues. The MSLN gene encodes a $70 \mathrm{kD}$ precursor protein that is cleaved into the $40 \mathrm{kD}$ cell-surface mature MSLN fragment and a 30 kD soluble fragment. Mature MSLN is detectable in nearly all mesotheliomas and approximately $30 \%$ of all cancers (29). Over-expression of MSLN in lung adenocarcinomas is correlated with decreases in both overall and relapse-free survival (16). Although neither the normal or pathological role of MSLN is explicitly known, it has been suggested that MSLN may promote the development of malignancy by affecting cell 
adherence (41), cell survival and proliferation (16, 19, 41), invasion and migration (16), apoptosis resistance, and chemosensitivity (41). The soluble MSLN fragment is detectable in the serum of many cancer patients, and its presence has been investigated as a potential biomarker for lung adenocarcinoma, mesothelioma (37), pancreatic cancer $(19,41)$, and ovarian cancer (12).

Since MSLN is thought to play a role in carcinogenesis, this study was designed to determine the functional role of MSLN in SWCNT-transformed bronchial epithelial cells (BSW). A stably-transfected MSLN knockdown line (BSW shMSLN) was generated and used to evaluate the effects of MSLN knockdown on cell migration, invasion, colony formation, and tumor sphere formation in vitro, as well as tumorigenesis and metastasis in vivo. $\mathrm{A}$ MSLN overexpressing BEAS-2B line, B2B/MSLN, was also generated to determine if MSLN overexpression alone could induce the same in vitro malignant phenotypic changes as those previously noted in the BSW cells. The mechanism by which MSLN could contribute to these behaviors was investigated using Ingenuity Pathway Analysis, which suggested that MSLN induced cyclin E, leading to cell cycle dysregulation. The effects of MSLN on cyclin E and cell cycle regulation were verified experimentally.

\subsection{Results}

\subsubsection{Mesothelin expression is increased in human lung tumors and lung cancer} cell lines. As reports on the pervasiveness of MSLN in human cancers vary widely, we first verified that MSLN was detectable in human lung tumors lysates. MSLN expression was assessed in ten human lung tumor samples and corresponding adjacent, healthy 
tissue controls (Fig. 1A). Samples included six large cell carcinomas, three squamous cell carcinomas, and one adenocarcinoma. None of the control samples had notable MSLN expression. However, five of the ten tumor samples had increased MSLN expression, ranging from two to tenfold more MSLN than the corresponding normal controls (Fig. 1B).

To validate the differential expression of MSLN in malignant versus non-malignant cell lines, MSLN expression was assessed in non-cancerous BEAS-2B cells, two lung cancer lines (A549, H460), and BSW cells, the previously generated SWCNTtransformed human bronchial epithelial line (47) (Fig. 1C). BEAS-2B cells had a lower level of MSLN expression than both the BSW cells and the lung cancer lines, A549 (adenocarcinoma) and H460 (large cell carcinoma) (Fig. 1D). Even though the BSW cells

originated from the non-cancerous BEAS-2B cells, their MSLN expression was on par with the cancer cell lines.

\subsubsection{MSLN knockdown BSW cells are less aggressive than BSW cells in}

vitro and in vivo. To determine the functional role of MSLN in the expression of a more malignant phenotype by BSW cells, BSW cells were stably transfected with either MSLN short-hairpin (sh)RNA (shMSLN) or a vector control (shC). Western blot analysis (Fig. 2, A and B) showed that MSLN was decreased substantially in the shMSLN-transfected cells (BSW shMSLN). MSLN was decreased to nearly undetectable levels in two clones (BSW shMSLN-1 and BSW shMSLN-5) and these clones were selected for further study.

Colony formation on soft agar is used to assess anchorage-independent cell growth in vitro, a hallmark of malignant transformation (5). MSLN knockdown cells (BSW 
shMSLN) formed significantly fewer colonies on soft agar than BSW controls (BSW shC) $(23.7 \pm 5.1$ vs. $53.0 \pm 9.9)$ and the colonies that did form were smaller than those formed by the BSW controls (Fig. 2, C and E). Although non-cancerous cells are not usually expected to form colonies on soft agar, it has been noted that BEAS-2B cells form small, slow-growing colonies despite their non-malignant origins $(40,45)$. Previously, it was shown that BSW cells formed eight times more colonies than passage-matched BEAS2B cells (47).

Tumor spheres form from the proliferation of cancer stem cells and the number of tumor spheres that form is indicative of the relative amount of cancer stem cells in the culture (2). BSW shMSLN cells formed significantly fewer tumor spheres than BSW shC $(50.2 \pm 10.9$ vs. $81.0 \pm 9.7)$ and the spheres formed by the BSW shMSLN cells were overall smaller than those formed by BSW ShC (Fig. 2, D and F).

To determine if the reduction in colony formation was due specifically to the decrease in MSLN, the experiments were repeated with a MSLN-overexpressing line. BEAS-2B cells were transiently transfected to overexpress MSLN (B2B/MSLN) (Fig. 2G). After MSLN overexpression was verified, B2B/MSLN colony formation on soft agar was assessed. B2B/MSLN formed substantially more colonies than B2B cells $(6.5 \pm 2.17$ vs $0.17 \pm 0.51$ ), and the colonies that formed were overall much larger than those seen with B2B cells (Fig. 2, $\mathrm{H}$ and I). Next, the effects of MSLN on cell migration and invasion were assessed. Migration was quantified using a Transwell ${ }^{\circledR}$ system with $8 \mu \mathrm{m}$ pores in the barrier. Cells that crossed the pores in the barrier were stained and counted (Fig. 3, A and $\mathrm{C}$ ). The BSW vector control cells (BSW shC) had 2.6 times as many migrated cells 
as the MSLN knockdown cells (BSW shMSLN). Previously, BSW cells were shown to migrate twice as much as passage-matched BEAS-2B controls (47).

Invasion was quantified in a similar manner to migration, except a thin layer of extra-cellular matrix was added to form a barrier over the pores in the Transwell $^{\circledR}$ insert. In order for cells to cross through the barrier, they must first break down the extra-cellular matrix (18). BSW shMSLN demonstrated significantly (2.8 times) less invasion than BSW shC (Fig. 3, B and D). Previously, BSW cells were shown to invade three times as much as passage-matched BEAS-2B controls. Overall, the invasion and migration assays show that the reduction in MSLN significantly reduces the invasion and migration abilities of the BSW cells.

Next, the effect of MSLN overexpression on migration and invasion was assessed using B2B/MSLN cells. B2B/MSLN cells demonstrated significantly increased migration and invasion relative to B2B controls. Migration was increased 6-fold (Fig. 3, E and G) relative to B2B controls, while invasion was increased 4-fold (Fig. F and $\mathrm{H}$ ).

Since decreasing MSLN reduced the aggressiveness of BSW cells in vitro, we next assessed whether this translated to less severe in vivo behavior. While non-cancerous cells, including the parental BEAS-2B line, are not expected to form tumors in nude mice (30), the transformed BSW cells have been shown to form injection-site tumors in vivo (47). In our study, both BSW shMSLN and BSW shC cells formed injection-site tumors in mice after 30 days, with the BSW shC cells forming tumors three times larger than those resulting from BSW shMSLN cells (Fig. 4, A and B).

We also assessed the ability of BSW tumors to metastasize by measuring surface tumor nodules in the abdominal and thoracic cavities. BSW shMSLN cells formed 15 times fewer 
metastatic nodules (average of 1 per mouse) than BSW shC cells (Fig. 4C). Mice injected with BSW shC had significantly greater tumor surface area in pulmonary and hepatic cross sections ( $56 \pm 31.27$ vs $2.25 \pm 4.5 \mathrm{~cm}^{2}$ ) (Fig. 4D). Examination of hematoxylin and eosin stained liver and lung slices confirmed these findings, with tumor nodules present in the liver and lungs of mice treated with BSW shC cells but not in mice treated with BSW shMSLN cells (Fig. 4E). Tissue sections were stained with anti-human mitochondrial and anti-human MSLN antibodies to ensure that the tumors had originated from the injected human cells (Fig. 4, F and G). Tissue staining results indicate that liver and lung metastatic lesions were of human origin and high in MSLN expression.

\subsubsection{Mesothelin increases cyclin E, which drives more rapid cell}

proliferation. Possible mechanisms behind MSLN-driven malignancies were investigated by using Ingenuity Pathway Analysis (IPA) to determine the overlap between gene expression changes in BSW cells, as previously determined by whole-genome mRNA microarray (23), and known relationships with over-expressed MSLN (Fig. 5). The prediction suggests that activated TNF, and $\mathrm{K}$-ras $(Z=2.03)$, mediators commonly increased with lung cancer, coupled with decreased and inhibited cyclin A, cyclin B and cyclin-dependent kinase 1 (CDK1) expression contribute to the induction of MSLN. Once MSLN has increased, it may increase the expression of matrix metalloproteinase (MMP) -7 and MMP-9, contributing to increased invasion, as well as stimulating increases in IL6, Stat3, and cyclin E, which contribute to cell cycle dysfunction. MSLN is predicted to have an inhibitory effect on both p53 and the pro-apoptotic mediator BAX. Inhibition of p53 would complement the dysfunctional cell cycle progression resulting from the 
increase in cyclin E. Suppression of the anti-apoptotic mediator BAX may shift the cells towards a more apoptosis-resistant state.

From the IPA data, it appears that MSLN induces cyclin E, which regulates cell cycle progression by acting as a gatekeeper at the $\mathrm{G} 1 / \mathrm{S}$ phase transition. The IPA prediction was verified using Western blotting, which confirmed that cyclin E was highly expressed in BSW shC cells but barely detectable in either BSW shMSLN clone (Fig. 6, A and $B$ ), indicating that MSLN is an upstream regulator of cyclin $E$ in these cells.

Increases in cyclin $\mathrm{E}$, and the subsequent decreases in time spent in $\mathrm{G} 1$, can result in more rapid cell cycle progression and ultimately increased proliferation rates. The change in proliferation rate can be used to determine the functional significance of the changes in cyclin E and cell cycle distribution. A colorimetric proliferation assay, which allows for the determination of the number of metabolically active cells based on their conversion of the reagents tetrazolium salt to a water-soluble formazan dye, was used to quantify cell proliferation (Fig. 6C). By day 3, BSW shMSLN cells proliferated at half the rate of the BSW shC cells. In addition, BSW cells proliferate 2-3 times faster than passage-matched BEAS-2B controls (47). Thus, knocking down MSLN nearly reverts cells to their original growth rate.

Flow cytometry was carried out to determine if the change in cyclin E levels correlated with a change in cell cycle distribution. Compared to the BSW shC cells, the BSW shMSLN cells had a greater percentage of the population in G2 phase and fewer cells progress to $S$ phase (Fig. 7). Since cyclin E regulates the G1/S phase transition, the increased S population in the BSW shC cells serves as supporting evidence that the 
increase in cyclin $E$ translates to a change in behavior. In addition, the decrease in the G2 population also indicates more rapid cell cycle progression.

\subsection{Discussion}

Cancer develops from continuous dysregulation of the mechanisms controlling critical cell behaviors. Chemical and physical stimuli can contribute to both the initial cellular damage and the propagation of those effects. Assessing the potential carcinogenicity and carcinogenic mechanisms of new materials is often challenging due to the long latent periods between exposure and disease onset and the intricate molecular pathways regulating cell behavior (22). Rapid assessment of toxicity, as is possible with acute exposure models, does not always capture the full range of toxicological implications, particularly when used to assess bio-persistent materials like asbestos and CNTs $(42,48)$.

CNTs have been likened to asbestos in terms of their fibrous structure and durability. As their integration into consumer products grows, understanding their toxicity has become a pressing public health matter $(10,42,44)$. Their small, light, easily aerosolized structure makes inhalation exposures, and thus eventual lung toxicity, a primary concern (32). Once inhaled, SWCNTs can deposit in the deepest regions of the lung, the broncho-alveolar junctions and the alveoli themselves, where they persist for up to several months $(22,48)$. After an initial inflammatory response and the generation of reactive oxygen species (ROS), SWCNT persistence in the lung leads to the formation of granulomas and interstitial fibrosis $(7,35,42,48)$. Prolonged pulmonary inflammation and fibrosis increase the risk of developing lung cancer $(22,34)$. Additionally, SWCNTs may 
further increase the risk by damaging DNA and interfering with cell replication $(7,22,34)$. Reported SWCNT genotoxicity, in in vitro and in vivo models, includes aneuploidy, centrosome fragmentation, damage to mitotic spindles, and increased numbers of micronuclei $(22,34,36)$. Moreover, long term exposure to CNTs induced malignant transformation of bronchial and small airway epithelial cells $(47,48)$. SWCNT exposure in a mouse inhalation model also resulted in K-ras oncogene mutations, which have been implicated in carcinogenesis $(34,35)$. Despite the evidence for SWCNT-induced DNA damage, their status as an oncogenic initiator is still unconfirmed (48). However, a murine inhalation study, using an initiator/promotor model, demonstrated that MWCNTs, specifically Mitsui-7 MWCNT, do act as tumor promotors (32).

To better understand the molecular mechanisms contributing to CNT carcinogenicity, we investigated the role of MSLN in the malignant transformation of lung epithelial cells chronically exposed to SWCNT. MSLN was historically associated with mesothelioma (37), which is primarily an asbestos-induced malignancy (48). MSLN has since identified in nearly $30 \%$ of all cancers, including a large number of lung (16), pancreatic $(19,41)$ and ovarian carcinomas (12).

The role of MSLN in lung cancer is not well defined, at least in part because its expression is highly variable even among patients with the same type and grade of tumor. For lung adenocarcinomas, MSLN has been detected in $39-83 \%$ of tumors $(13,16,25-$ 27), and tumor MSLN expression may be an independent predictor of relapse-free and overall survival (16). Increased MSLN expression is thought to be involved in cell adherence and chemotherapy resistance, as well as increased invasion and migration, cell proliferation, and anchorage-independent growth (41). However, as neither the 
physiologic role of MSLN nor its pathological mechanism is known, the varied prevalence of MSLN in tumors has made it difficult to discern whether or not MSLN has a functional role in the development of cancer.

Our BSW line, generated by exposing BEAS-2B cells to low doses of SWCNTs for 6 months (47), offers a model to study the molecular and behavioral changes associated with malignant transformation of lung epithelial cells. This exposure model has been used to study the effects of chronic heavy metal exposures $(28,39)$ as well as chronic exposures to single $(20,21,47,48)$ and multiwall CNTs $(44,48)$. In addition, this model was used to discern the differences in the toxicological effects of chronic in vitro exposure of lung epithelial cells to SWCNTs, MWCNTs, and asbestos, all of which are known to accumulate and trigger inflammation and fibrosis in vivo, versus ultrafine carbon black, which exhibits lower in vivo toxicity and is not highly fibrogenic (48). Chronic in vitro exposure to non-toxic levels of SWCNTs, MWCNTs, and asbestos triggered neoplastic transformation, while cells exposed to ultrafine carbon black maintained a non-neoplastic phenotype with reduced cell proliferation, fewer colonies on soft agar, and an increase in genome-wide cell death signaling.

In the case of BSW cells, abnormal cell morphology was noted within the first 4 weeks of exposure and abnormal cell behavior was characterized after 6 months of exposure (47). Although the mechanisms behind some aspects of this transformation, including the acquired apoptosis resistance $(30,47)$ and stem-like characteristics $(21,23)$, have been investigated, they do not account for all of the changes seen in the BSW cells, suggesting that there are likely additional, currently unidentified, contributing mechanisms. In this study, we found that BSW cells have greater MSLN expression than the parental BEAS- 
$2 \mathrm{~B}$ cells and this model provided a unique platform to study the role of MSLN in malignant transformation and subsequent aggressive behavior.

BSW cells are morphologically and behaviorally distinct from the parental BEAS-2B line. These changes trend towards an overall more aggressive phenotype, including increased cell proliferation, invasion, migration, colony formation on soft agar, acquired resistance to apoptosis, induction of angiogenesis, and the ability to form tumors in vivo (47). These changes are consistent with those seen in other in vitro chronic CNT exposures $(20,44,48)$. Interestingly, many of the changes seen in BSW cells correspond with previously suggested roles of MSLN.

In the present study, MSLN appears to contribute significantly to the increased metastatic potential of BSW cells. Metastases are responsible for the overwhelming majority of cancer deaths, because they are difficult to prevent or treat. After MSLNknockdown, invasion and migration were reduced significantly. Conversely, migration and invasion were increased when MSLN overexpression was induced in BEAS-2B cells. Lung cancer cells transduced to overexpress MSLN have previously been shown to have a two-fold increase in both migration and invasion (16), which is similar to the 2.6 and 2.8 fold increase we noted in the BSW shC cells relative to the BSW shMSLN cells. MSLNrelated increases in invasion and migration have also been reported in pancreatic cancer (16), ovarian cancer (6), and mesothelioma (24) cell lines.

Anchorage-independent growth, an in vitro hallmark of carcinogenic transformation, was also affected by changes in MSLN expression. We found that knocking down MSLN decreased soft agar colony and tumor sphere formation, while MSLN overexpression increased soft agar colony formation. This is in line with previous reports that MSLN- 
expressing breast cancer cells formed 1.5 times more colonies, and the colonies were twice the size, compared to non-MSLN expressing controls (43). Similarly, silencing MSLN in mesothelioma cells result in reductions in both the number and size of colonies that form on soft agar (24).

A reduction in the aggressiveness of malignant cells in vitro should correlate with reduced tumor formation, both local and metastatic, in vivo. Our results show that knockdown of MSLN in our BSW cells did translate to significantly less metastases and reduced (but not eliminated) primary tumors in vivo. Immunohistochemistry indicated that metastatic lesions in BSW shC treated mice were of human origin and expressed human MSLN. A previous study using pancreatic cancer cells that stably overexpressed MSLN showed that the MSLN overexpressing cells resulted in larger tumors than the parental line (3). In rats with tuberous sclerosis, knocking out MSLN reduced the formation of kidney tumors, a change that was partially attributed to the MSLN-knockdown cells being less able to bind to collagen (50).

Although elucidation of the specific mechanism for the decrease in metastases is beyond the scope of this study, it is likely that MSLN mediates cell-cell adhesion, and its overexpression aids the circulating tumor cells in attaching to new locations. This has been previously described in the context of ovarian cancer metastases attaching to the peritoneal lining via interactions between MSLN and the ovarian cancer antigen CA-125 (31). Additionally, in a kidney tumor model, MSLN knockout cells were less adherent to collagen (50). How this translates to other forms of cancer is not yet known, but further studies of this interaction in other lines, including BSWs, have the potential to enhance our understanding of the metastatic process. 
The mechanism by which MSLN mediates metastasis may be related to its normal function. MSLN is typically found on the apical surface of mesothelial linings $(27,31)$ and is often (but not exclusively) seen on the apical surface or leading edge of tumors $(1,8$, $9,33)$. This is similar to the localization and effects of CA-125, the ovarian cancer antigen, and may explain why the co-expression of MSLN and CA-125 results in worse outcomes than either alone (31). This might also point towards MSLN having a similar function to CA-125.

Predictions as to the molecular mechanisms underlying MSLN-driven changes in BSW cell behavior were investigated using IPA. These predictions were based on increased MSLN in BSW cells and fit well with our experimental observations as well as previously reported effects of MSLN in other cancer cell lines. Many of the predictions correlated with the observed aggressive behaviors, such as increases in MMP-9, which would contribute to the increased invasiveness of our SWCNT-transformed BSW cells. MSLN-induced increases in MMP have previously been linked to increased invasiveness in ovarian cancer cells (6).

One notable relationship is that TNF appears to induce MSLN, which in turn increases IL-6. IL-6 is known to stimulate TNF, creating a potential positive feedback loop that could markedly increase both MSLN and IL-6. IL-6 is an inflammatory chemokine that may serve as an important intermediate between MSLN over-expression and MSLNinduced aggressive behaviors. Specifically, IL-6 promotes cell growth, chemo-resistance, anchorage-independent cell growth, and invasiveness $(4,17)$ and is over-expressed in MSLN-expressing pancreatic cancer cells $(4,41)$. It appears likely that MSLN does not 
directly induce IL-6; rather, MSLN activates NF-kB, which in turn induces IL-6 production (3).

Additionally, higher levels of MSLN tend to correlate with signs of inflammatory responses, including the induction of IL-6 (3), degradation of extracellular matrix by matrix metalloproteinases (6), and changes in attachment mediators $(15,50)$. Our IPA analysis also suggests that MSLN induces TNF-a, a key pro-inflammatory cytokine with an important role in lung cancer development. Typically an immune or inflammatory response to tissue damage or foreign particles will resolve as the particles clear and the environment returns to normal. The bio-persistence of CNTs, however, would trigger a continuous inflammatory response, which can form a positive feedback loop to further increase MSLN expression. The similar bio-persistence of CNTs and asbestos could also explain why MSLN is increased in nearly all mesotheliomas.

Increases in MSLN expression were also predicted, by IPA, to increase cyclin E, a cell cycle regulator that promotes the G1/S phase transition and is frequently dysregulated in cancer cells (14). We demonstrated that MSLN induced cyclin E in BSW cells. Elevated cyclin E has been found in pancreatic cells transformed to over-express MSLN, where it was suggested that MSLN induces cyclin E through an IL-6/Stat3 pathway (3). This is a plausible mechanism for the increase in cyclin E in the BSW cells as well, particularly considering that IPA predicted MSLN-induced increases in both IL-6 and cyclin E.

In addition to verifying that MSLN knockdown did exhibit decreased cyclin E expression, we found that knockdown of MSLN shifted the proportion of cells in G2 and S phase of the cell cycle. After MSLN and, by extension, cyclin E knockdown, the 
expected decrease in S phase population was coupled with an increase in the G2 population. MSLN knockdown in mesothelioma cells also resulted in a decrease of the $S$ phase population (24). IPA suggests that decreased cyclins A and B expression coupled with inhibited CDK1, which promotes G1/S and G2/M transition, found in the BSW line independent of MSLN expression, potentially contributed to increases in G2 population. The increased cyclin E in the MSLN overexpressing cells may overcome the inhibitory effects of decreased cyclins A and B. This effect would be reversed when MSLN, and consequently cyclin $\mathrm{E}$, expression is reduced.

As cyclin $E$ drives cell cycle progression via $\mathrm{G} 1 / \mathrm{S}$ transition, increased cyclin $\mathrm{E}$ is expected to increase cell proliferation. We found that, while BSW cells proliferate three times faster than BEAS-2B cells, knocking down MSLN in the BSW cells reduced their proliferation rate 2.5 times, nearly to the rate of the BEAS-2B cells. Similarly, $\mathrm{H} 1299$ lung cancer cells transduced to express MSLN proliferated 1.6 times faster than controls (16). MSLN overexpression has also been shown to increase the proliferation rate of pancreatic cancer cells $(3,24)$.

While we focused primarily on the contribution of cyclin E to the cell cycle dysregulation seen in BSW cells, our IPA results suggest that MSLN overexpression can affect additional cell proliferation and survival regulatory mechanisms. Specifically, MSLN may inhibit both p53 and the pro-apoptotic mediator BAX. Dysregulation of p53, a critical cell cycle regulator, is common in cancers. Typically p53 regulates G1/S cell cycle checkpoint, halting entry into the cell cycle when genetic damage is present (46). Inhibition of p53, such as that induced by MSLN, would allow cell proliferation regardless of genetic integrity. We have previously found that p53 is dysregulated in BSW cells (21, 
47). The combined loss of p53-mediated damage control and cyclin E-driven increases in proliferation is amenable to the rapid accumulation of further mutations, which could manifest in vivo as more aggressive or treatment-resistant disease.

Additionally, inhibition of pro-apoptotic mediators, such as BAX, shifts the cell towards an anti-apoptotic state. Although our analysis did not indicate changes in apoptotic regulators other than BAX, studies in pancreatic cancer cells suggest that MSLN overexpression leads to the decreases in both BAX and Bad (also pro-apoptotic), as well as increases in the anti-apoptotic mediators Bcl-2 and Mcl-1 (41). BSW cells also exhibit apoptotic resistance, particularly in response to extrinsic mediators of apoptosis with BAX playing a potentially important signaling role (30).

Overall, we found that knocking down MSLN in the BSW cells reduced the severity of the malignant behaviors tested but did not completely eliminate them. This suggests that MSLN is important in the formation of both primary tumors and metastases but is not the sole mechanism behind their formation. Even though MSLN may not be the primary driver of cancerous transformation, it appears to be heavily involved in a number of processes contributing to the final malignant phenotype and may make an attractive target for cancer therapeutics. 


\subsection{Materials and Methods}

3.5.1 Patient tumor samples. Human lung tissue lysates were purchased from Protein Biotechnologies (Ramona, CA). Samples from lung tumors and adjacent healthy tissue were provided as pairs. Six large cell carcinomas, two squamous carcinomas, and one adenocarcinoma were tested.

3.5.2 Cell Culture. Non-tumorigenic human bronchial epithelial BEAS-2B cells were cultured with SWCNT $\left(0.02 \mu \mathrm{g} / \mathrm{cm}^{2}\right)$ for 6 months to generate CNT-transformed bronchial epithelial cells (BSW), as previously described $(21,47)$. This cell model has been reported to be an appropriate model for in vitro lung carcinogenesis studies $(28,48)$. BSW cells were maintained in Dulbecco's modified Eagle medium (DMEM) supplemented with 5\% fetal bovine serum (FBS), $2 \mathrm{mM}$ L-glutamine, 100 units $/ \mathrm{mL}$ penicillin and $100 \mu \mathrm{g} / \mathrm{mL}$ streptomycin (Gibco, Gaithersburg, MA). A549 cells, human lung carcinoma alveolar type II epithelial cells, were cultured in DMEM supplemented with $5 \%$ fetal bovine serum (FBS), 2 mM L-glutamine, 100 units $/ \mathrm{mL}$ penicillin and $100 \mu \mathrm{g} / \mathrm{mL}$ streptomycin (Gibco, Gaithersburg, MA). Non-small cell lung cancer H460 cells were cultured in RPMI 1640 medium supplemented with 5\% FBS, $2 \mathrm{mM}$ L-glutamine, and 100 units $/ \mathrm{mL}$ penicillin/streptomycin. With the exception of laboratory-generated BSW and passage control BEAS-2B cells, all cells were purchased from ATCC (Manassas, VA). All cells were maintained in a humidified atmosphere of $5 \% \mathrm{CO}_{2}$ at $37^{\circ} \mathrm{C}$. 
3.5.3 Generation of stable MSLN knockdown cells. Stable MSLN knockdown BSW cells and vector-transfected control cells were generated by using shMSLN lentiviral plasmid vector or scrambled shRNA vector (OriGene Technologies, Inc, Rockville, MD) and transfected using the Amaxa Nucleofector II electroporation method (Lonza, Walkersville, MD). Stable transfected single clones were selected with $5 \mu \mathrm{g} / \mathrm{mL}$ of puromycin. MSLN knockdown was verified by Western blotting.

3.5.4 Transient MSLN overexpression in B2B cells. MSLN overexpression was transiently induced in BEAS-2B cells using plasmid DNA (pEasy-MSLN-iCre-HA-Flag, plasmid \#31305, Addgene, Cambridge, MA) and FuGENE HD transfection reagent (Promega, Madison, WI), according to the manufacturers protocol. At $24 \mathrm{~h}$ after the transfection, MSLN protein expression levels were assessed by Western blotting. MSLNoverexpressing BEAS-2B cells (B2B/MSLN) were used in migration, invasion, and colony formation assays.

3.5.5 Cell proliferation. MSLN knockdown and vector control cells were seeded at a density of $1.5 \times 10^{4}$ cells per well in $100 \mu \mathrm{L}$ of media in a 96-well plate (Fisher, Waltham, MA). After 24, 48, or 72 hours, $20 \mu \mathrm{L}$ of CellTiter 96 Aqueous One Solution (Promega, Madison, WI) were added to each well, and the cells were incubated at $37^{\circ} \mathrm{C}$ for an additional 3 hours. Viable cells cleave the reagent's tetrazolium salt to a soluble formazan dye, resulting in a color change proportional to the number of live cells. Absorbance was measured at $490 \mathrm{~nm}$, with a reference wavelength at $630 \mathrm{~nm}$, using a BioTek Plate Reader (BioTek, Winooski, VT). 
3.5.6 Soft agar colony formation assay. BSW shMSLN and BSW shC cells ( 2500 cells) were suspended in $0.5 \mathrm{~mL}$ of culture medium and mixed with equal amount of $0.7 \%$ agar to a final agar concentration of $0.35 \%$. The $1 \mathrm{~mL}$ cell/agar suspensions were immediately plated onto a 6 -well plate coated with $0.5 \%$ agar in culture medium $(1 \mathrm{~mL} /$ well). Colonies were examined under a light microscope after 2 weeks of culture. Colonies were counted if $>50$ cells. In order to assess the self-renewing property of cells, colonies were collected by gentle centrifugation, dissociated into single cell suspensions, filtered and cultured under conditions described above (second colony formation).

3.5.7 Tumor sphere formation assay. Tumor sphere assay was performed under stemcell selective (non-adherent and serum-free) conditions as previously described (49). Briefly, $5 \times 10^{3}$ cells were suspended in $0.8 \%$ methylcellulose (MC)-based serum-free medium (Stem Cell Technologies, Vancouver, Canada) supplemented with $20 \mathrm{ng} / \mathrm{mL}$ of epidermal growth factor (BD Biosciences, San Jose, CA), basic fibroblast growth factor and $4 \mathrm{mg} / \mathrm{mL}$ of insulin (Sigma, St. Louis, MO) in an ultra-low adherent 6-well plate. Cells were then cultured for two weeks. Tumor spheres were examined under a light microscope. In order to assess the self-renewing property of cells, spheres were collected by gentle centrifugation, dissociated into single cell suspensions, filtered and cultured under conditions described above (second sphere formation).

3.5.8 Cell migration and invasion assays. In vitro cell migration was determined using a 24-well Transwell ${ }^{\circledR}$ unit with polycarbonate (PVDF) filters (8 $\mu \mathrm{m}$ pore size). In vitro cell invasion was performed with a BD Matrigel ${ }^{\circledR}$ invasion chamber (BD Biosciences, San Jose, CA). Briefly, cells at the density of $1.5 \times 10^{4}$ cells per well (migration) or $3 \times 10^{4}$ cells 
per well (invasion) were seeded into the upper chamber of the Transwell ${ }^{\circledR}$ unit in serumfree medium. The lower chamber of the unit was filled with a normal growth medium containing $5 \%$ FBS. Chambers were incubated at $37{ }^{\circ} \mathrm{C}$ in a $5 \% \mathrm{CO}_{2}$ atmosphere for 48 hours. The non-migrating or non-invading cells were removed from the inside of the insert with a cotton swab. Cells that migrated or invaded to the underside of the membrane were fixed and stained with Diff-Quik (Dade Behring, Newark, DE). Inserts were visualized and scored under a light microscope (Leica DM, IL). Number of migrating and invading cells were counted. Results represent the mean \pm SD from 10 fields evaluated.

3.5.9 Immunoblotting. Cells were washed twice with ice-cold PBS and lysed on ice with modified RIPA buffer containing protease and phosphatase inhibitor mixture (Roche Molecular Biochemicals, Indianapolis, IN) for 30 minutes. The lysate was sonicated briefly and centrifuged at $14,000 \mathrm{~g}$ for 20 minutes. Cell lysates ( $40 \mu \mathrm{g}$ of protein) were fractionated by $10 \%$ sodium dodecyl sulfate-polyacrylamide gel electrophoresis (SDSPAGE) and transferred onto polyvinylidene difluoride membranes (PVDF) (Bio-Rad Laboratories, Hercules, CA). The transferred membranes were blocked for 1 hour with $5 \%$ nonfat dry milk in TBST (25 mM Tris- $\mathrm{HCl}, \mathrm{pH} 7.4,125 \mathrm{mM} \mathrm{NaCl}, 0.05 \%$ Tween 20 ) followed by MSLN (ab96869, Abcam, Cambridge, MA) or cyclin E (Cell Signaling, Danvers, MA) primary antibody at $4{ }^{\circ} \mathrm{C}$ overnight with gentle shaking. Membranes were washed three times with TBST for 10 minutes each followed by incubation with a horseradish peroxidase-conjugated $\square$-actin secondary antibody (A5441, Sigma, St. Louis, MO) for 1 hour at room temperature. Protein bands were visualized using enhanced chemiluminescence detection reagents from Millipore (Millipore Corporation, 
Billerica, MA). Actin was blotted to ensure equal loading of the samples, and data were quantified using image $\mathrm{J}$ densitometry software.

3.5.10 Flow cytometry. MSLN-knockdown and scrambled shRNA control cells were seeded overnight in 6 well plates (Fisher, Waltham, MA) at a concentration of $3 \times 10^{5}$ cells/well. The cells were trypsinized, collected, washed twice with PBS, and fixed overnight in $70 \%$ ethanol (Fisher, Waltham, MA) at $-20^{\circ} \mathrm{C}$. Subsequently, the cells were washed and suspended in 0.2\% Tween 20 (Sigma, St. Louis, MO) PBS solution for 15 minutes at $37{ }^{\circ} \mathrm{C}$, followed by RNase $\mathrm{A}(180 \mu \mathrm{g} / \mathrm{mL})$ for 15 minutes at room time temperature. The cells were then stained with propidium iodide PBS $(50 \mu \mathrm{g} / \mathrm{mL}$; Sigma) for 15 minutes at room temperature. Changes in DNA content were determined using a BD LSR Fortessa Flow cell analyzer (BD Biosciences, San Jose, CA) and BD FACS express 5 software. The forward scatter (FSC) and side scatter (SSC) were used to gate the majority of the cell population; 20,000 events were collected for each sample. The selection of the cells was based on knowing that in the G0/G1 phase (before DNA synthesis) cells have a defined amount of DNA (i.e., a diploid chromosomal DNA content) and double that amount in the $\mathrm{G} 2$ or $\mathrm{M}$ phase (G2/M, i.e., a tetraploid chromosomal DNA content). During the $S$ phase (DNA synthesis), cells contain between one to two DNA levels.

3.5.11 Tumor xenograft mouse models. Animal care and experimental procedures described in this study were performed in accordance with the Guidelines for Animal Experiments at West Virginia University with the approval of the Institutional Animal Care 
and Use Committee (IACUC \# 15-0702). Immunodeficient NOD/SCID gamma mice,

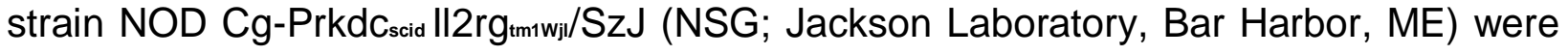
maintained under pathogen-free conditions within the institutional animal facility. Food and tap water were given ad libitum. Mice (6 per group) were subcutaneously injected with $5 \times 10^{5}$ cells of BSW with shMSLN or shControl stable knockdown cells suspended in $100 \mu \mathrm{L}$ of ExtraCel ${ }^{\circledR}$ hydrogel (Advanced BioMatrix, San Diego, CA). Mice were inspected daily for any signs of distress such as weight loss, hunching, failure to groom, or red discharge from the eyes. After 30 days, mice were euthanized and tumors were dissected and weighted. Metastatic nodules were counted from the surface on the intestine, liver and lungs. Liver and lung tumor specimens were dissected into $5 \mu \mathrm{m}$ sections and stained with hematoxylin and eosin (H\&E) to confirm cancer histology and metastasis in organs. All tissue sectioning and staining were performed at the West Virginia University Pathology Laboratory for Translational Medicine.

3.5.12 Immunostaining. Lung and liver sections in paraffin were deparaffinized and rehydrated. Antigens were retrieved with $10 \mathrm{mM}$ sodium citrate solution in the microwave for 20 minutes. The slides were then blocked with 3\% BSA $0.1 \%$ Tween in 1 X PBS blocking buffer for $1 \mathrm{~h}$ and were incubated with anti-human MSLN antibody (Abcam, Cambridge, MA) at a dilution of 1:500 or anti-human mitochondria antibody (EMD Millipore Corporation, Temecula, CA ) at a dilution of $1: 100$ overnight at $4{ }^{\circ} \mathrm{C}$. After washing with PBS three times, the slides were incubated with biotinylated secondary antibodies for an hour, followed by avidin-biotin complex $(A B C)$ reagent (Vector Laboratories, Inc, Burlingame, CA), and detected with DAB kit (Vector Laboratories, Inc, 
Burlingame, CA). After color development, the slides were counterstained with hematoxylin, dehydrated, and mounted with Permount mounting medium (Fisher Scientific, Waltham, MA). Images were taken using a light microscope with the SimplePCI 6 software (Compix Inc, Cranberry, PA).

3.5.13 Ingenuity Pathway Analysis. To further understand MSLN's role in promoting the observed cancer cell phenotype, we examined the potential signaling pathways within BSW cells. Whole genome mRNA microarray data from our previous work (NCBI GEO Accession \#GSE56104) was uploaded into Ingenuity Pathway Analysis (Qiagen). All known mRNA and miRNA signaling associations with MSLN were plotted along with differential gene expression of BSW vs. passage control BEAS-2B cells (30). Genes and miRNA were excluded from the network if the relationship with MSLN was not reported in lung tissue. Due to the complexity of the network and aggressive, metastatic ability of BSW cells, genes only known to play a role in metastatic and Stage IV lung cancer were kept in the MSLN network. Lastly, predictive activation/inhibition analysis of both upstream and downstream genes from MSLN was conducted using Z-scores. Based on the observed significant MSLN protein over-expression, we evaluated upstream and downstream targets with a predicted MSLN activation. These predictions were overlaid on the MSLN signaling network if $Z \pm 2$.

3.5.14 Statistics. Results were expressed as means \pm SD. All values were derived from at least three independent experiments. Differences between groups were assessed by 
analysis of variance (ANOVA) followed by Student's $t$ test. For all analyses, two-sided $P$ values of $\leq 0.05$ were considered statistically significant.

\subsection{Acknowledgements}

This work was supported by grants from the National Institutes of Health (R01-ES022968, R01-EB018857), National Science Foundation (CBET-1434503), and WVU Cancer Institute (Sara C. Allen and James F. Allen Comp Lung Cancer Research Fund). Flow cytometric analysis was performed in the West Virginia University Flow Cytometry Core Facility, which is supported in part by the NIH Grant P30 GM103488. Imaging experiments and image analysis were performed in the West Virginia University Microscope Imaging Facility, which is supported by the West Virginia University Cancer Institute and NIH grants P20 RR016440, P30 RR032138/GM103488 and P20 RR016477.

\section{Disclaimer}

The findings and conclusions in this report are those of the authors and do not necessarily represent the views of the National Institute for Occupational Safety and Health.

\section{Disclosures}

No potential conflicts of interest to disclose.

\section{Author Contributions}

X.H. conducted most experiments and analyzed data; E.D. conducted some experiments, analyzed data, and drafted the manuscript; T.A.S. performed IPA analysis; A.C. assisted in lung 
carcinogenesis studies; V.C. involved in the discussion and preparation of the manuscript;

C.Z.D. provided assistance in nanomaterial characterization and cell interaction studies; L.W.

provided assistance in long-term CNT exposure and assay development; and Y.R. coordinated and involved in all aspects of the project. 


\subsection{Figures and Tables}

Figure 3.1
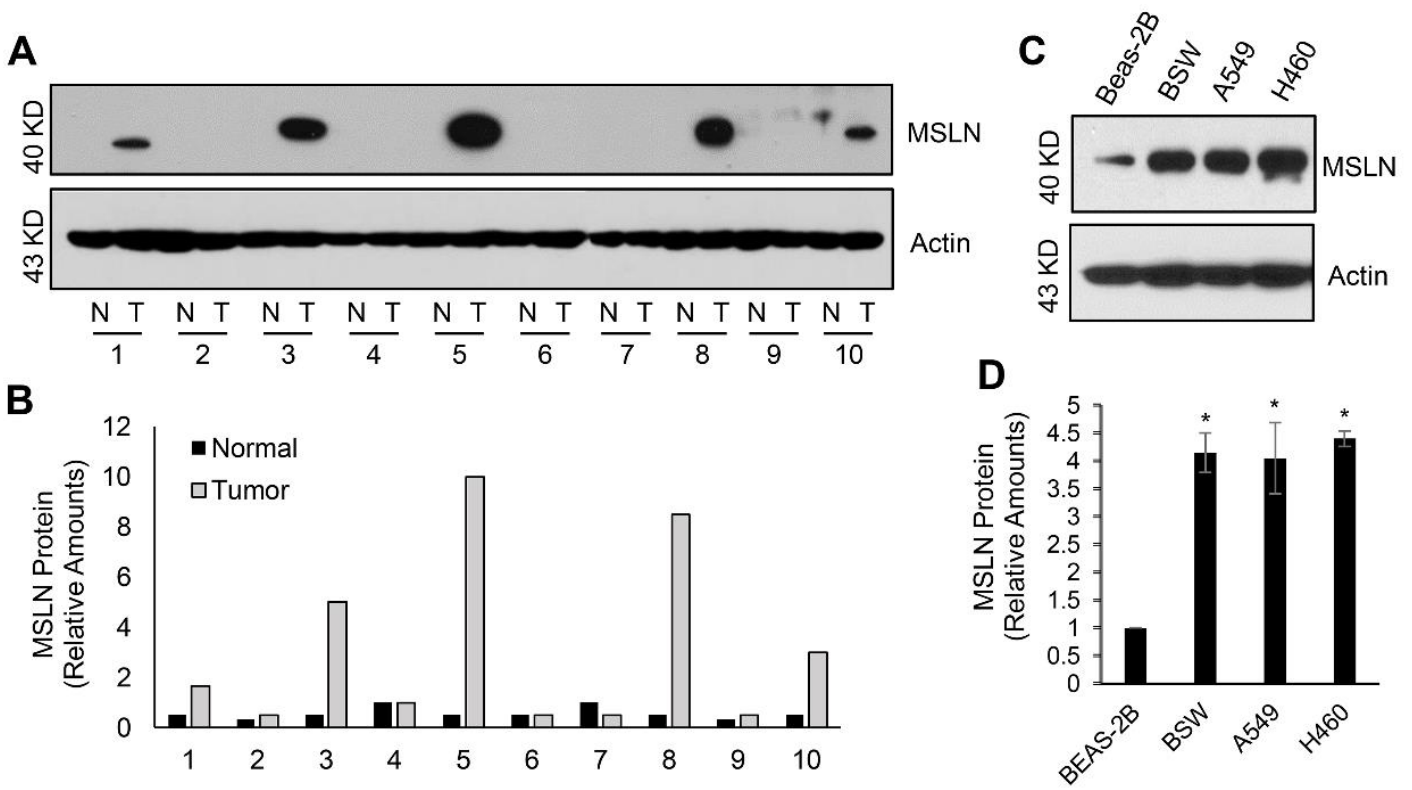

Figure 3.4. MSLN is overexpressed in lung cancer. (A) Western blot for MSLN in pairs of lung tumor lysates $(\mathrm{T})$ and normal tissue controls $(\mathrm{N})$ from the same patients. Pairs 16 are large cell carcinomas, pairs $7-9$ are squamous cell carcinomas, and pair 10 is adenocarcinoma. (B) Quantification of tumor MSLN expression, relative to actin. (C) Western blot and (D) quantification of MSLN in non-cancerous bronchial epithelial cells (BEAS-2B), CNT-transformed BSW cells, and in two established lung cancer cell lines (A549 and H460). ${ }^{*} \mathrm{P}<0.05$ vs. BEAS-2B. 
Figure 3.2
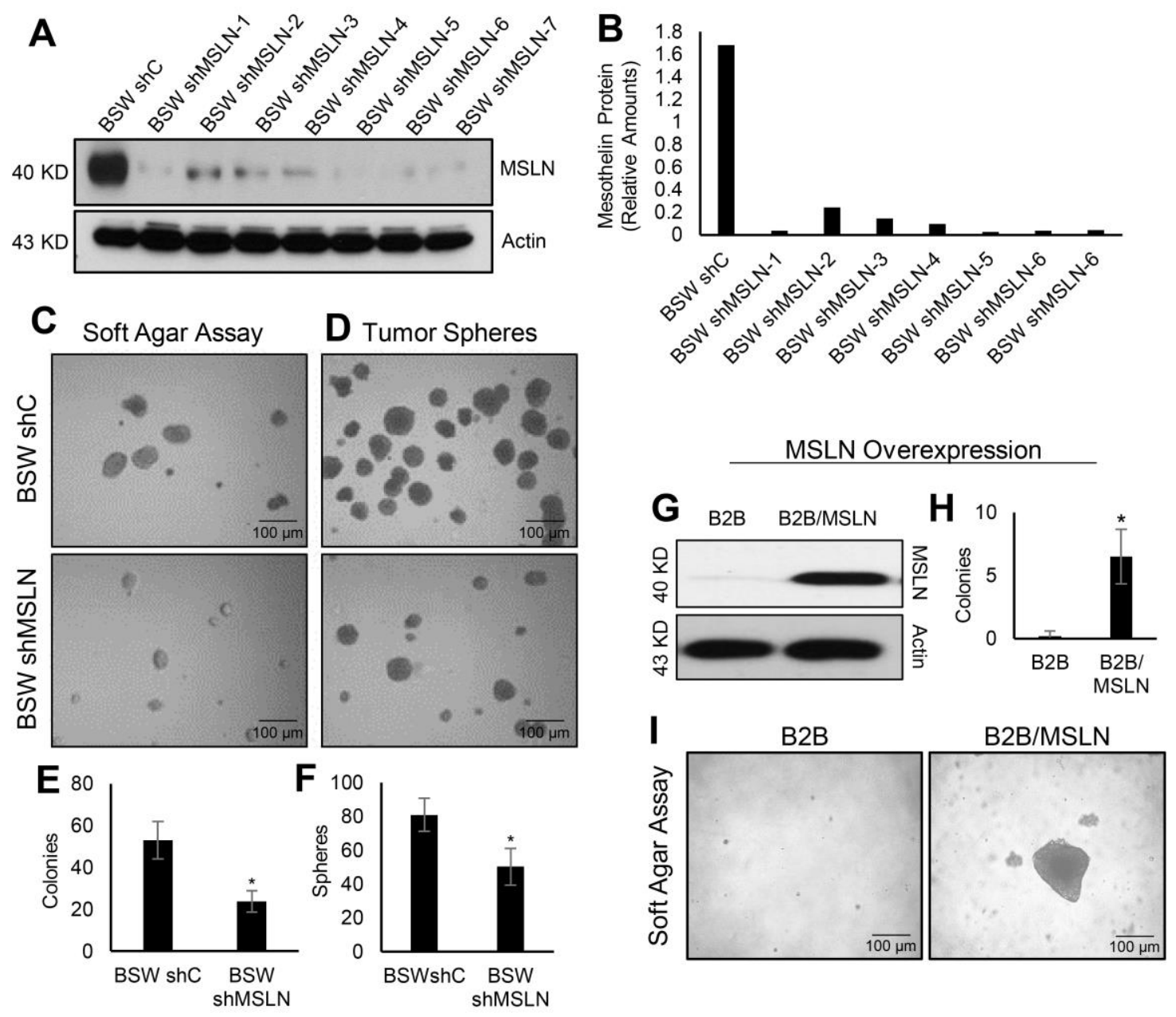

Figure 3.5. Knockdown MSLN reduces soft agar colony and tumor sphere formation. (A) Western blot and (B) quantification of MSLN in several stable knockdown clones. (C) Representative images showing colony formation on soft agar of BSW shC and BSW shMSLN cells. (D) Representative images of tumor sphere formation of BSW shC and BSW shMSLN cells. (E) Quantification of colonies formed by BSW shC and BSW shMSLN cells. (F) Quantification of tumor spheres formed by BSW shC and BSW shMSLN cells. ${ }^{*} \mathrm{P}<0.05$ vs. BSW shC. (G) Western blot of MSLN expression in B2B and B2B/MSLN cells. (H) Quantification and (I) representative images of colonies formed by B2B and B2B/MSLN cells. ${ }^{*} \mathrm{P}<0.05$ vs. B2B. 

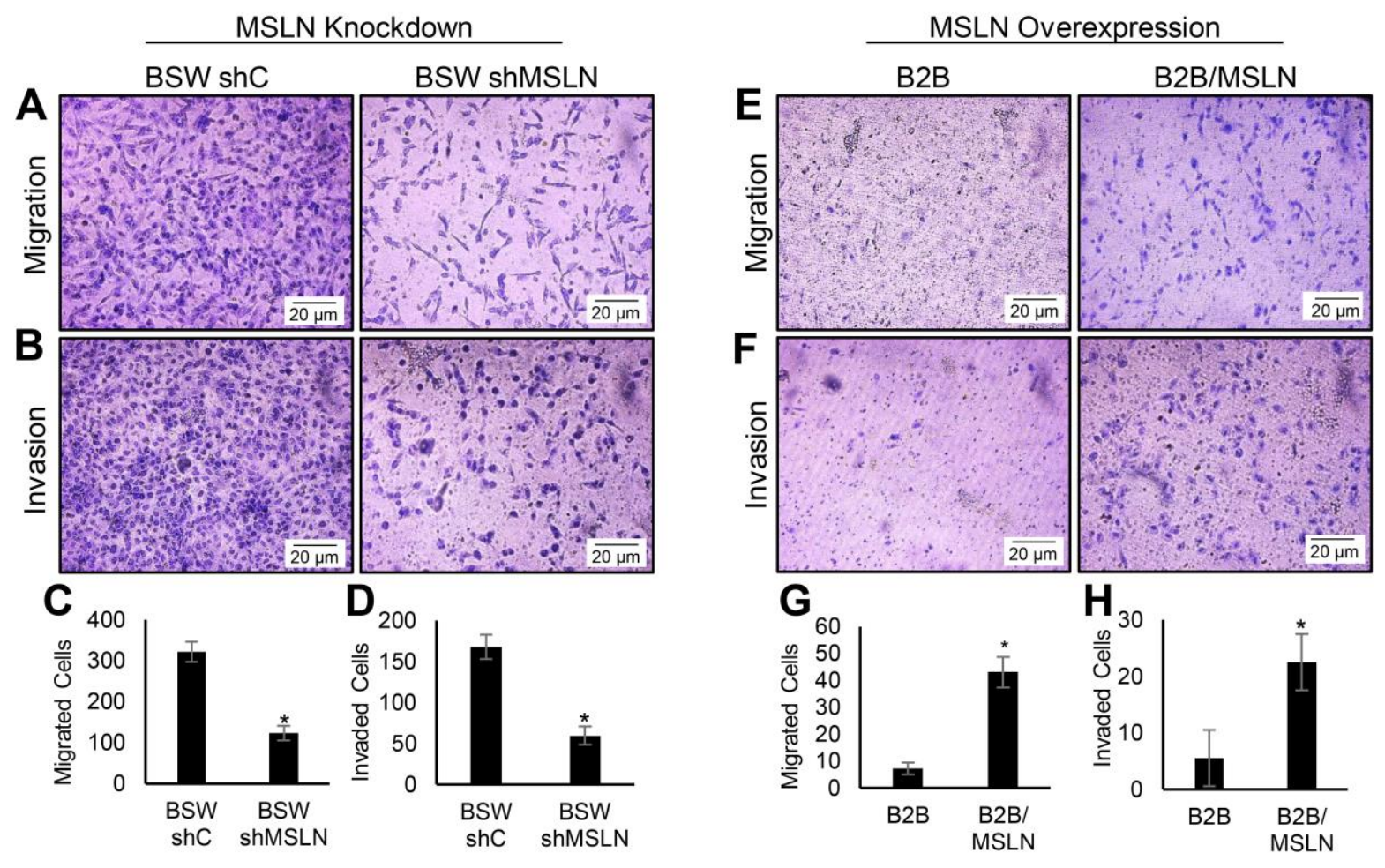

Figure 3.6. MSLN expression increases migration and invasion in vitro. (A) Representative images of BSW shC and BSW shMSLN migration and (B) invasion, stained with Diff-Quik. (C) Quantification of migrating BSW shC and BSW shMSLN cells. (D) Quantification of invading BSW shC and BSW shMSLN cells. ${ }^{*} \mathrm{P}<0.05$ vs. BSW shC. (E) Representative images of B2B and B2B/MSLN migration and $(F)$ invasion, stained with Diff-Quik. (G) Quantification of migrating B2B and B2B/MSLN cells. (H) Quantification of invading B2B and B2B/MSLN cells. ${ }^{*} \mathrm{P}<0.05$ vs. B2B. 
Figure 3.4
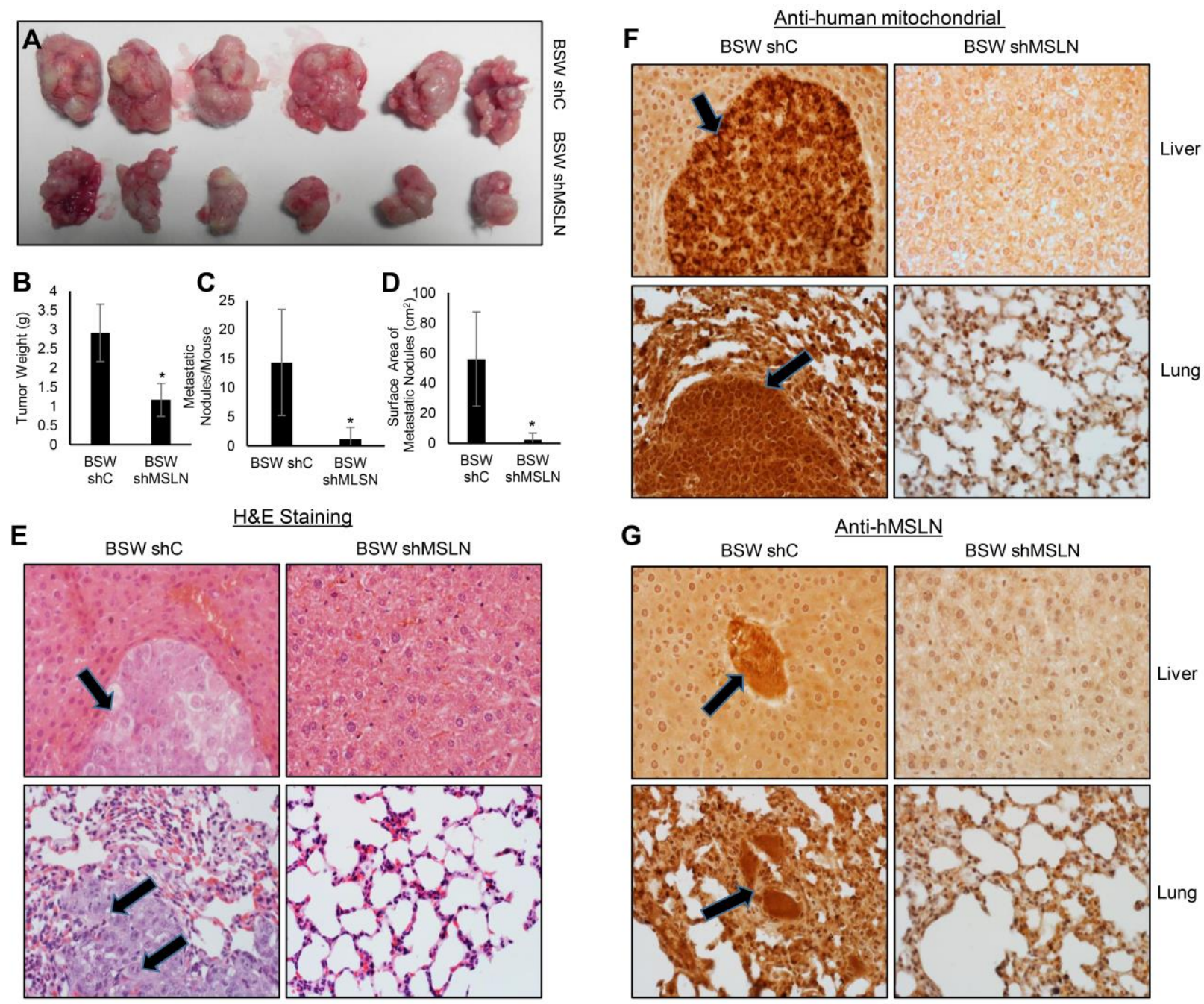

Figure 3.7. MSLN regulates tumor growth and metastasis in vivo. (A) Injection site tumors. (B) Graphical representation of injection-site tumor weights. (C) Number of thoracic and abdominal surface metastatic nodules. (D) Surface area of hepatic and pulmonary metastases. (E) Representative pulmonary and hepatic tissues with hematoxylin and eosin (H\&E) staining. Arrows denote metastatic tumor nodules. $(F)$ Immunostaining of human mitochondria and $(G)$ human MSLN in representative liver and lung sections of mice injected with BSW shC or BSW shMSLN. Arrows denote metastatic tumor nodules. ${ }^{*} \mathrm{P}<0.05$ vs. BSW shC. 


\section{Figure 3.5}

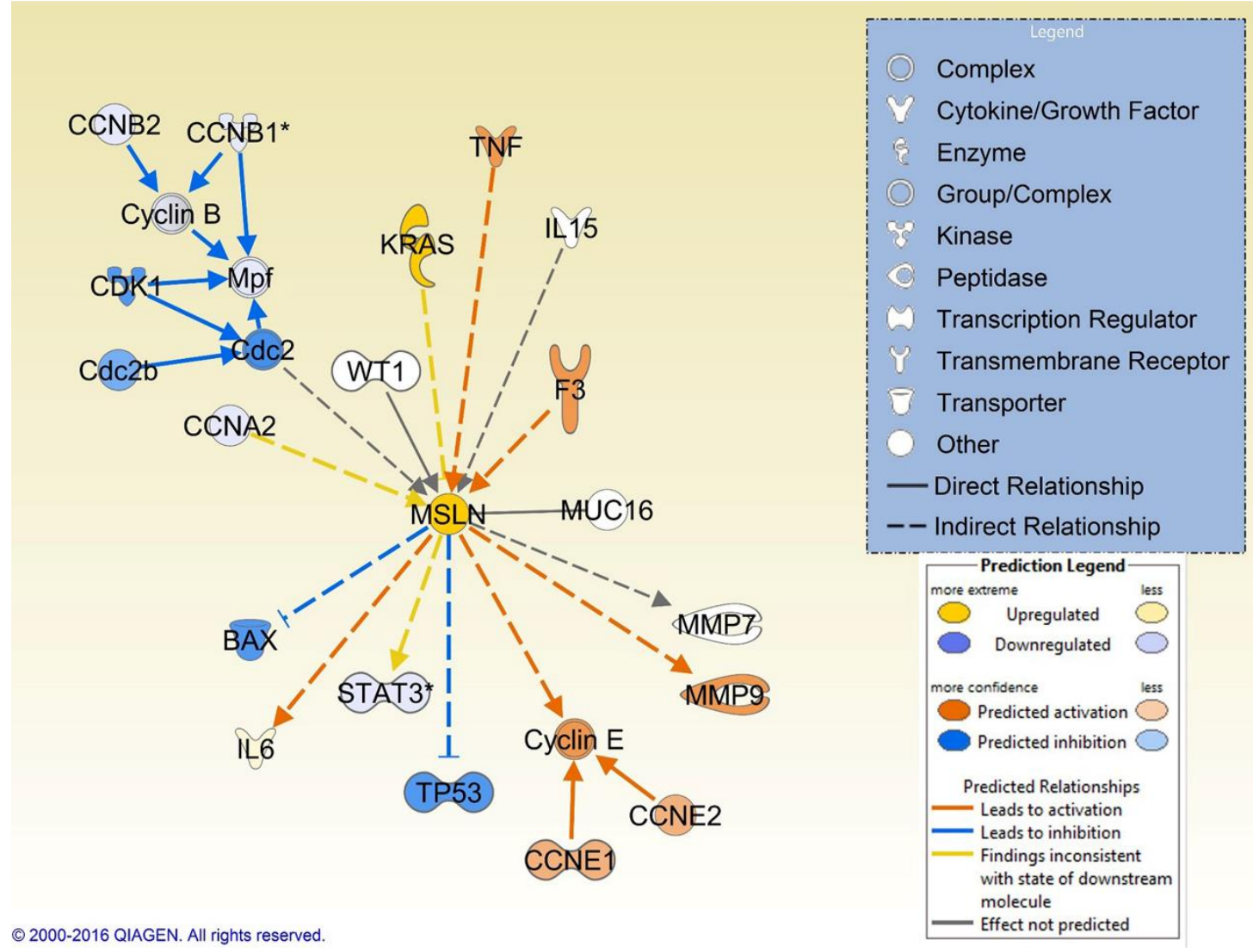

Figure 3.8. Changes in gene expression when MSLN is increased in BSW cells. Ingenuity Pathway Analysis (IPA) was used to predict differentially expressed genes. Yellow to orange colors represent up-regulation, while blue represent down-regulation, compared to BEAS-2B passage-matched controls. Color intensity signifies fold change. 
Figure 3.6

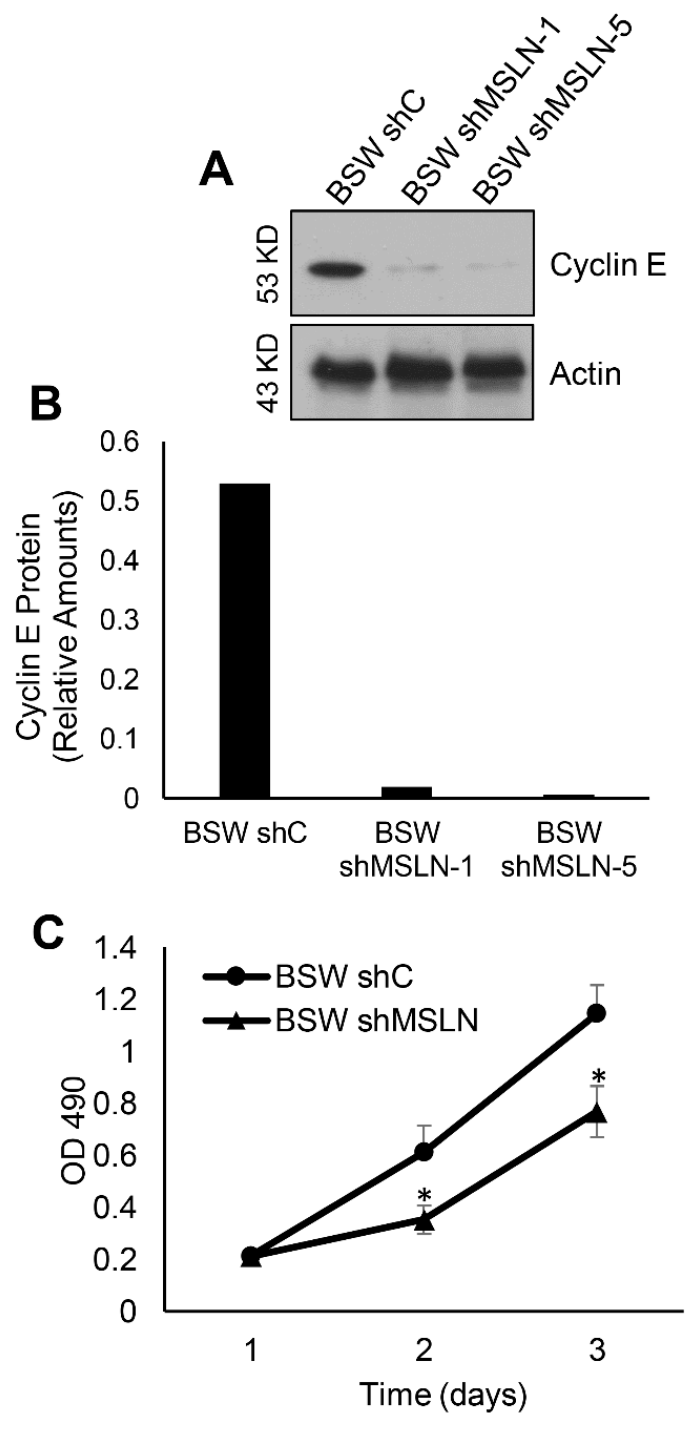

Figure 3.9. MSLN regulates cyclin E and cell proliferation. (A) Western blot and (B) quantification of cyclin E in BSW shC and two MSLN knockdown clones. (C) Proliferation of BSW shC and BSW shMSLN cells, determined using Promega Aqueous One-Step viability assay, which measures mitochondrial metabolic rate, at 24 and 48 hours after seeding. ${ }^{*} \mathrm{P}<0.05$ vs. BSW shC. 
Figure 3.7
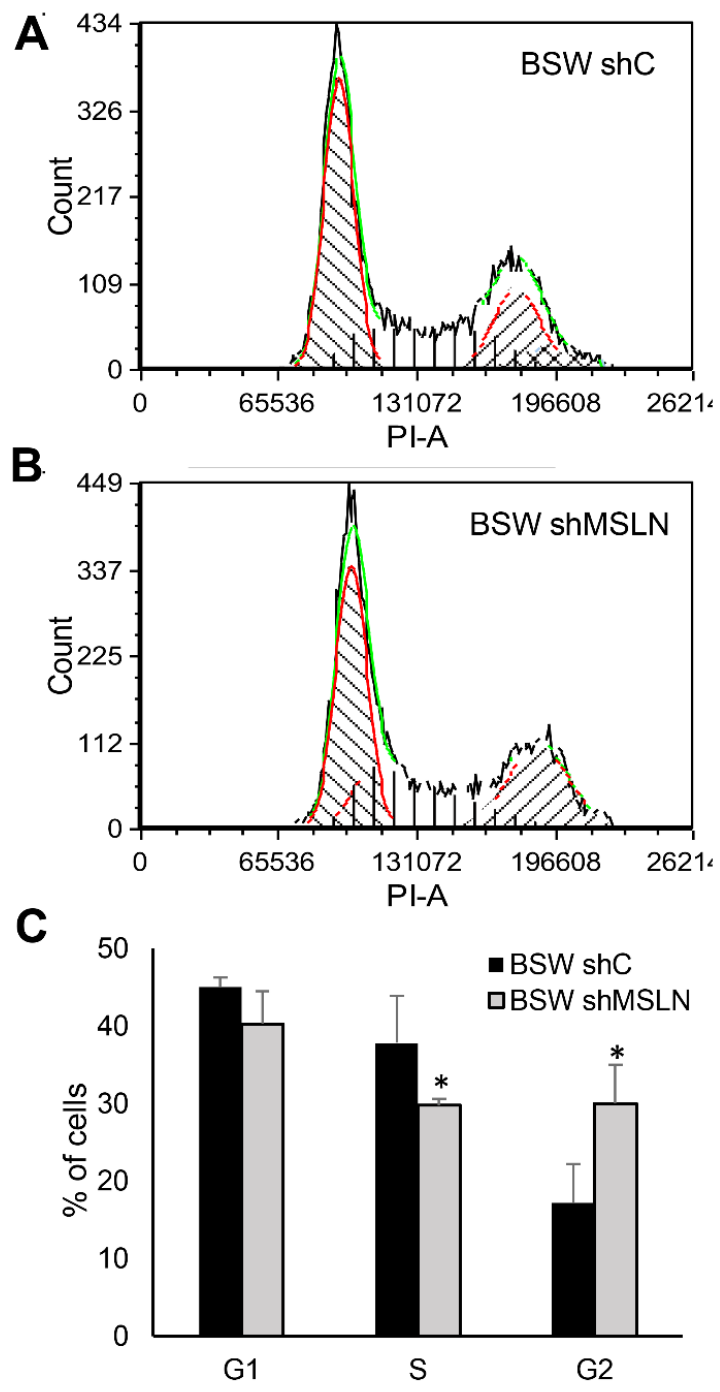

Figure 3.10. Cell cycle progression is affected by MSLN. (A) Representative flow cytometry plot for propidium iodide stained BSW shC and (B) BSW shMSLN cells. (C) Graphical representation of cell cycle distribution, determined from flow cytometry. FACS Express 5 software was used for analysis and proliferation statistics (green and red traces). ${ }^{*} \mathrm{P}<0.05$ vs. BSW shC. 


\subsection{References}

1. Alvarez H, Rojas PL, Yong K-T, Ding H, Xu G, Prasad PN, Wang J, Canto M, Eshleman JR, Montgomery EA, Maitra A. Mesothelin is a specific biomarker of invasive cancer in the Barrett-associated adenocarcinoma progression model: translational implications for diagnosis and therapy. Nanomedicine 4: 295-301, 2008.

2. Beck B, Blanpain C. Unravelling cancer stem cell potential. Nat Rev Cancer 13: 727-738, 2013.

3. Bharadwaj U, Li M, Chen C, Yao Q. Mesothelin-induced pancreatic cancer cell proliferation involves alteration of cyclin $\mathrm{E}$ via activation of signal transducer and activator of transcription protein 3. Mol Cancer Res 6: 1755-1765, 2008.

4. Bharadwaj U, Marin-Muller C, Li M, Chen C, Yao Q. Mesothelin overexpression promotes autocrine IL-6/sIL-6R trans-signaling to stimulate pancreatic cancer cell proliferation. Carcinogenesis 32: 1013-1024, 2011.

5. Borowicz S, Van Scoyk M, Avasarala S, Karuppusamy Rathinam MK, Tauler J, Bikkavilli RK, Winn RA. The soft agar colony formation assay. J Vis Exp : 16, 2014.

6. Chang MC, Chen CA, Chen PJ, Chiang YC, Chen YL, Mao TL, Lin HW, Lin Chiang WH, Cheng WF. Mesothelin enhances invasion of ovarian cancer by 
inducing MMP-7 through MAPK/ERK and JNK pathways. Biochem J 442: 293302, 2012.

7. Chen D, Stueckle TA, Luanpitpong S, Rojanasakul Y, Lu Y, Wang L. Gene expression profile of human lung epithelial cells chronically exposed to singlewalled carbon nanotubes. Nanoscale Res Lett 10, 2015.

8. Drapkin R, Crum CP, Hecht JL. Expression of candidate tumor markers in ovarian carcinoma and benign ovary: Evidence for a link between epithelial phenotype and neoplasia. Hum Pathol 35: 1014-1021, 2004.

9. Erdogan E, Demirkazik FB, Gulsun M, Ariyurek M, Emri S, Sak SD. Incidental localized (solitary) mediastinal malignant mesothelioma. Br J Radiol 78: 858-861, 2005.

10. Fisher C, Rider AE, Jun Han Z, Kumar S, Levchenko I, Ostrikov K. Applications and nanotoxicity of carbon nanotubes and graphene in biomedicine. J. Nanomater (2012).

11. Gangwal AS, Brown JS, Wang A, Houck KA, Dix DJ, Kavlock RJ, Hubal EAC. Informing selection of nanomaterial concentrations for ToxCast in vitro testing based on occupational exposure potential. Environmental Health Perspectives 119: $1539-1546$.

12. Hassan R, Remaley AT, Sampson ML, Zhang J, Cox DD, Pingpank J, Alexander R, Willingham M, Pastan I, Onda M. Detection and quantitation of 
serum mesothelin, a tumor marker for patients with mesothelioma and ovarian cancer. Clin Cancer Res 12: 447-453, 2006.

13. Ho M, Bera TK, Willingham MC, Onda M, Hassan R, FitzGerald D, Pastan I. Mesothelin expression in human lung cancer. Clin cancer Res 13: 1571-1575, 2007.

14. Hwang HC, Clurman BE. Cyclin E in normal and neoplastic cell cycles. Oncogene 24: 2776-2786, 2005.

15. Ito $T$, Kajino K, Abe M, Sato K, Maekawa H, Sakurada M, Orita H, Wada R, Kajiyama Y, Hino O. ERC/mesothelin is expressed in human gastric cancer tissues and cell lines. Oncol Rep 31: 27-33, 2014.

16. Kachala SS, Bograd AJ, Villena-Vargas J, Suzuki K, Servais EL, Kadota K, Chou J, Sima CS, Vertes E, Rusch VW, Travis WD, Sadelain M, Adusumilli PS. Mesothelin overexpression is a marker of tumor aggressiveness and is associated with reduced recurrence-free and overall survival in early-stage lung adenocarcinoma. Clin cancer Res 20: 1020-1028, 2014.

17. Kipps E, Tan DSP, Kaye SB. Meeting the challenge of ascites in ovarian cancer: new avenues for therapy and research. Nat Rev Cancer 13: 273-282, 2013.

18. Kramer N, Walzl A, Unger C, Rosner M, Krupitza G, Hengstschläger M, Dolznig H. In vitro cell migration and invasion assays. Mutat Res 752: 10-24, 2013. 
19. Li M, Bharadwaj U, Zhang R, Zhang S, Mu H, Fisher WE, Brunicardi FC, Chen C, Yao Q. Mesothelin is a malignant factor and therapeutic vaccine target for pancreatic cancer. Mol Cancer Ther 7: 286-296, 2008.

20. Lohcharoenkal W, Wang L, Stueckle TA, Dinu CZ, Castranova V, Liu Y, Rojanasakul Y. Chronic exposure to carbon nanotubes induces invasion of human mesothelial cells through matrix metalloproteinase-2. ACS Nano 7: 77117723, 2013.

21. Luanpitpong S, Wang L, Castranova V, Rojanasakul Y. Induction of stem-like cells with malignant properties by chronic exposure of human lung epithelial cells to single-walled carbon nanotubes. Part Fibre Toxicol 11: 22-40, 2014.

22. Luanpitpong S, Wang L, Davidson DC, Riedel H, Rojanasakul Y. Carcinogenic Potential for High Aspect Ratio Carbon Nanomaterials. Environ Sci: Nano 3:483493, 2016.

23. Luanpitpong S, Wang L, Stueckle TA, Tse W, Chen YC, Rojanasakul Y. Caveolin-1 regulates lung cancer stem-like cell induction and p53 inactivation in carbon nanotube-driven tumorigenesis. Oncotarget 5: 3541-3554, 2014.

24. Melaiu O, Stebbing J, Lombardo Y, Bracci E, Uehara N, Bonotti A, Cristaudo A, Foddis R, Mutti L, Barale R, Gemignani F, Giamas G, Landi S. MSLN gene silencing has an anti-malignant effect on cell lines overexpressing mesothelin deriving from malignant pleural mesothelioma. PLoS One 9: e85935, 2014. 
25. Miettinen M, Sarlomo-rikala M. Expression of calretinin, thrombomodulin, keratin 5 , and mesothelin in lung carcinomas of different types an immunohistochemical analysis of 596 tumors in comparison with epithelioid mesotheliomas of the pleura. Am J Surg Pathol 27: 150-158, 2003.

26. Ordóñez NG. The immunohistochemical diagnosis of mesothelioma a comparative study of epithelioid mesothelioma and lung adenocarcinoma. Am J Surg Pathol 27: 1031-1051, 2003.

27. Ordóñez NG. Application of mesothelin immunostaining in tumor diagnosis. Am J Surg Pathol 27: 1418-1428, 2003.

28. Park Y-H, Kim D, Dai J, Zhang Z. Human bronchial epithelial BEAS-2B cells, an appropriate in vitro model to study heavy metals induced carcinogenesis. Toxicol Appl Pharmacol 287: 240-245, 2015.

29. Pastan I, Hassan R. Discovery of mesothelin and exploiting it as a target for immunotherapy. Cancer Res 74: 2907-2912, 2014.

30. Pongrakhananon V, Luanpitpong S, Stueckle TA, Wang L, Nimmannit U, Rojanasakul Y. Carbon nanotubes induce apoptosis resistance of human lung epithelial cells through FLICE-inhibitory protein. Toxicol Sci 143: 499-511, 2015. 
31. Rump A, Morikawa Y, Tanaka M, Minami S, Umesaki N, Takeuchi M, Miyajima A. Binding of ovarian cancer antigen CA125/MUC16 to mesothelin mediates cell adhesion. J Biol Chem 279: 9190-9198, 2004.

32. Sargent LM, Porter DW, Staska LM, Hubbs AF, Lowry DT, Battelli L, Siegrist KJ, Kashon ML, Mercer RR, Bauer AK, Chen BT, Salisbury JL, Frazer D, McKinney W, Andrew M, Tsuruoka S, Endo M, Fluharty KL, Castranova V, Reynolds SH. Promotion of lung adenocarcinoma following inhalation exposure to multi-walled carbon nanotubes. Part Fibre Toxicol 11, 2014.

33. Shimizu A, Hirono S, Tani M, Kawai M, Okada KI, Miyazawa M, Kitahata Y, Nakamura Y, Noda T, Yokoyama S, Yamaue H. Coexpression of MUC16 and mesothelin is related to the invasion process in pancreatic ductal adenocarcinoma. Cancer Sci 103: 739-746, 2012.

34. Shvedova AA, Yanamala N, Kisin ER, Tkach A V, Murray AR, Hubbs A, Chirila MM, Keohavong P, Sycheva LP, Kagan VE, Castranova V. Long-term effects of carbon containing engineered nanomaterials and asbestos in the lung: one year postexposure comparisons. Am J Physiol Lung Cell Mol Physiol 306: L170-82, 2014.

35. Shvedova AA, Kisin E, Murray AR, Johnson VJ, Gorelik O, Arepalli S, Hubbs AF, Mercer RR, Keohavong P, Sussman N, Jin J, Yin J, Stone S, Chen BT, Deye G, Maynard A, Castranova V, Baron PA, Kagan VE. Inhalation vs. aspiration of single-walled carbon nanotubes in C57BL/6 mice: inflammation, 
fibrosis, oxidative stress, and mutagenesis. Am J Physiol Lung Cell Mol Physiol 295: L552-565, 2008.

36. Siegrist KJ, Reynolds SH, Kashon ML, Lowry DT, Dong C, Hubbs AF, Young S-H, Salisbury JL, Porter DW, Benkovic S a, McCawley M, Keane MJ, Mastovich JT, Bunker KL, Cena LG, Sparrow MC, Sturgeon JL, Dinu CZ, Sargent LM. Genotoxicity of multi-walled carbon nanotubes at occupationally relevant doses. Part Fibre Toxicol 11, 2014.

37. Smolková P, Nakládalová M, Zapletalová J, Jakubec P, Vildová H, Kolek V, Petřek M, Nakládal Z. Validity of mesothelin in occupational medicine practice. Int J Occup Med Environ Health 29, 2016.

38. Stella GM. Carbon nanotubes and pleural damage: perspectives of nanosafety in the light of asbestos experience. Biointerphases 6: P1-17, 2011.

39. Stueckle TA, Lu Y, Davis ME, Wang L, Jiang B-H, Holaskova I, Schafer R, Barnett JB, Rojanasakul Y. Chronic occupational exposure to arsenic induces carcinogenic gene signaling networks and neoplastic transformation in human lung epithelial cells. Toxicol Appl Pharmacol 261: 204-216, 2012.

40. Sun H, Clancy HA, Kluz T, Zavadil J, Costa M. Comparison of gene expression profiles in chromate transformed BEAS-2B cells. PLoS One 6: e17982, 2011.

41. Tang Z, Qian M, Ho M. The role of mesothelin in tumor progression and targeted therapy. Anticancer Agents Med Chem 13: 276-280, 2013. 
42. Thurnherr T, Brandenberger C, Fischer K, Diener L, Manser P, MaederAlthaus X, Kaiser J-P, Krug HF, Rothen-Rutishauser B, Wick P. A comparison of acute and long-term effects of industrial multiwalled carbon nanotubes on human lung and immune cells in vitro. Toxicol Lett 200: 176-186, 2011.

43. Uehara N, Matsuoka Y, Tsubura A. Mesothelin promotes anchorageindependent growth and prevents anoikis via extracellular signal-regulated kinase signaling pathway in human breast cancer cells. Mol cancer Res 6: 186-193, 2008.

44. Vales G, Rubio L, Marcos R. Genotoxic and cell-transformation effects of multiwalled carbon nanotubes (MWCNT) following in vitro sub-chronic exposures. $J$ Hazard Mater 306: 193-202, 2015.

45. Veljkovic E, Jiricny J, Menigatti M, Rehrauer H, Han W. Chronic exposure to cigarette smoke condensate in vitro induces epithelial to mesenchymal transitionlike changes in human bronchial epithelial cells, BEAS-2B. Toxicol Vitr 25: 446453, 2011.

46. Vermeulen K, Van Bockstaele DR, Berneman ZN. The cell cycle: a review of regulation, deregulation and therapeutic targets in cancer. Cell Prolif 36: 131-149, 2003.

47. Wang L, Luanpitpong S, Castranova V, Tse W, Lu Y, Pongrakhananon V, Rojanasakul Y. Carbon nanotubes induce malignant transformation and tumorigenesis of human lung epithelial cells. Nano Lett 11: 2796-2803, 2011. 
48. Wang L, Stueckle TA, Mishra A, Derk R, Meighan T, Castranova V, Rojanasakul Y. Neoplastic-like transformation effect of single-walled and multiwalled carbon nanotubes compared to asbestos on human lung small airway epithelial cells. Nanotoxicology 8: 485-507, 2014.

49. Wang Y, Yu Y, Tsuyada a, Ren X, Wu X, Stubblefield K, Rankin-Gee EK, Wang SE. Transforming growth factor- $\beta$ regulates the sphere-initiating stem celllike feature in breast cancer through miRNA-181 and ATM. Oncogene 30: 14701480, 2011.

50. Zhang D, Kobayashi T, Kojima T, Kanenishi K, Hagiwara Y, Abe M, Okura H, Hamano Y, Sun G, Maeda M, Jishage K, Noda T, Hino O. Deficiency of the Erc/mesothelin gene ameliorates renal carcinogenesis in Tsc2 knockout mice. Cancer Sci 102: 720-727, 2011. 


\section{Chapter 4}

\section{Project Insights and Future Directions}

\subsection{Design and Evaluation of Multi-functional SPION Platform}

Well-designed, multi-functional SPION platforms have the potential to improve cancer diagnostics and treatment. ${ }^{1-3}$ Initial studies of our therapeutic SPION platform, presented in Chapter 2, demonstrated the biocompatibility of our formulation and its potential for delivering ASO. Although these results were in line with previous reports on both accounts, our inability to consistently functionalize our platform presented a significant obstacle to biological testing.

Further work is needed to improve the reproducibility of the SPION-ASO coupling reaction (4.1.1 Optimization of SPION-DNA Coupling Procedure). The current procedures employ EDC, a carbodiimide coupling reagent that is widely used for biological coupling reactions. Despite its widespread use, EDC-mediated coupling reactions are notoriously difficult to optimize. ${ }^{4-9}$ More recently, other amide-forming biological linking agents have been developed to help overcome the limitations of EDC. ${ }^{10-15}$ Because consistent coupling methods are needed to move the SPION platform forward, ${ }^{16}$ these newer linking agents should also be considered.

Once the coupling process is consistent, the composition and physical properties of the conjugated SPIONs need to be fully characterized (4.1.2 Characterization of Functionalized SPION Platform). Our freshly synthesized SPIONs have been thoroughly 
evaluated, ${ }^{17}$ as have several batches of PAA-coated SPIONs. However, the final, ASOSPIONs have not been available in large enough quantities to evaluate.

Fully characterized SPIONs can then be introduced to biological systems (4.1.3 Evaluation of Biological Effects of SPION-ASO) and additional targeting mechanisms can be investigated (4.1.4 Selection of a Targeting Ligand to Improve the Selectivity of SPION Uptake by Survivin-Expressing Cancer Cells).

\subsubsection{Optimization of SPION-DNA Coupling Procedure}

There is little tolerance for formulation inconsistencies of therapeutic agents. Toxicity testing, and eventual clinical use, require careful control and characterization of all drug parameters. ${ }^{16}$ The proof-of-concept preliminary data presented in Chapter 2 supports the biocompatibility and therapeutic potential of our SPION-ASO conjugate. However, the attachment our amine-terminal ASO to the PAA coated SPION was inefficient and highly variable. ASO coupling efficiency was also directly related to the therapeutic efficacy of the SPION-ASO, even when doses were calculated to ensure equivalent ASO concentrations. The impact of the loading density of similarly arranged nucleic acids on NP biological effects has been previously described. ${ }^{25,26}$ Therefore, it is critically important that the SPION synthesis, preparation, and ligand loading be highly reproducible.

The development of a consistent, well-characterized SPION system will provide a platform that can be customized for other biological and research purposes. Although this 
specific SPION formulation was chosen as a cancer therapeutic, and thus targets the production of a cancer-specific protein, any number of amine-modified ligands can be conjugated to the PAA-coated SPION using similar methods. Combinations of targeting and therapeutic ligands would allow for highly specific in vivo delivery of drug-bearing SPIONs. ${ }^{27,28}$ Alternatively, ASO-SPIONs could be used for in vitro gene regulation, where they could serve as a gentler alternative to transfection. ${ }^{25}$

Therapeutic SPION platforms typically incorporate a polymer coating around SPION core to enhance biocompatibility and to provide functional groups for further functionalization. ${ }^{15,29-35}$ Our platform incorporates a $40 \%$ octylamine modified PAA, synthesized in-house, to serve this purpose ${ }^{36}$ Hydrophobic interactions are expected to drive an association between the PAA octylamine groups and the oleic acid surfactant that surrounds freshly synthesized SPIONs. The unmodified carboxylic acids of the PAA, which are hydrophilic, are expected to face outward and provide reactive sites for further modification. The SPION-PAA platform is intended to provide a versatile base to which biomolecules, including nucleic acids, aptamers, and antibodies, can be conjugated. Due to the role of the PAA coating in SPION functionalization, the first step in optimizing the coupling reaction will assess SPION-PAA consistency (4.1.1a Ensuring Particle Homogeneity).

Amide bonds, which are commonly used to covalently attach biomolecules, are possible when primary-amine containing ligands are introduced to the PAA surface. A coupling reagent is needed to drive amide bond formation, as direct condensation of the 
amine and carboxylic acid would require destructively high temperatures. ${ }^{37}$ Although there are a variety of coupling reagents available, the majority are not compatible with biomolecules. Many methods require the use of halogenated reagents, take place in organic solvents, or result in hazardous byproducts that are difficult to remove. Carbodiimide reagents, predominantly in the form of EDC, overcome many of the limitations of other coupling reagents and have become popular for covalent attachment of biological amines. However, EDC coupling is far from efficient and protocols often require extensive refinement (4.1.1b EDC-Mediated Amide Bond Formation). Newer carbodiimide reagents, including DMTMM, have emerged as more efficient and easier to use alternatives (4.1.1c Alternative Coupling Reagent: DMTMM).

\subsection{1a Ensuring Particle Homogeneity}

Based on our preliminary results, the inconsistency in our SPION-DNA platform was largely due to the DNA attachment reaction. The SPION platform is designed so that amine-terminal ligands, including ASO, can be covalently attached to the reactive carboxyl moieties on the surface of the PAA coated SPION. Thus, the issues with DNA attachment have two probable sources: improper orientation of the polymer coating used to transfer the SPIONs to aqueous solutions and ineffective coupling conditions.

To pinpoint the source of error, freshly synthesized and PAA-coated SPIONs were characterized determine their homogeneity. Uncoated SPIONs were had homogenous size distributions, as determined by TEM and DLS, and consistent composition by TGA. Composition analysis of PAA-coated SPIONs revealed that PAA consistently accounts 
for $75 \%$ of the mass of the coated SPIONs. Although the intention was to have the PAA form a single layer coating around the SPION, the composition analysis suggests that there are multiple layers of PAA, creating a thick coating. The orientation of this thick, multi-layer coating may contribute to the difficulty in attaching the DNA ligands as, if the carboxylic acids of the PAA are not facing outward, they will not be able to participate in the EDC-mediated covalent attachment of the DNA. However, it is likely that this is not the entire problem as some portion of the carboxylic acids in the outer layer of the polymer are facing outward since the PAA-coated SPIONs were dispersed in aqueous solutions, and alternative PAA orientations would result in exposed hydrophobic alkanes. Although it is not likely that the PAA coating is completely unable to be functionalized, further analysis may be required to ensure that it is reactive.

\subsection{1b EDC-Mediated Amide Bond Formation}

EDC is widely used for the formation of amides between biological molecules because it is water soluble, the excess reagent is easy to remove, and the reaction can take place at room temperature. ${ }^{15,38-42}$ Despite its widespread use, EDC reactivity and ideal conditions are poorly understood and some of its limitations contradict the major advantages of its use. ${ }^{42}$ For example, EDC can be used in aqueous systems, but interaction with water also leads to rapid hydrolysis of the EDC to inactive byproducts. ${ }^{13,38}$ In addition, EDC can cause rapid NP precipitation even at concentrations needed for the reaction to proceed. ${ }^{6,15,36}$ There is no standard protocol for EDC-mediated amide formation, and published procedures vary dramatically in terms of reaction time, 
temperature, EDC concentration, and coupling efficiency. ${ }^{4,10,15,39,42,43}$ Until recently, a lack of suitable alternatives contributed to the popularity of EDC in spite of its often poor coupling efficiency $y^{4,15,39,42,43}$ and the difficulty of optimizing reaction conditions. ${ }^{4-10}$

Optimization of the coupling reaction will first require PAA-coated SPIONs with consistent composition. The PAA-SPIONs should be fully coated, washed, filtered, and suspended in distilled water, PBS, or the chosen reaction buffer. It is important that SPIONs remain stably suspended in the reaction solution, both to improve coating efficiency and to reduce particle aggregation. SPION suspension can be improved initially by filtering the PAA-SPIONs and, later, by adjusting the reaction $\mathrm{pH} .{ }^{36}$ However, $\mathrm{pH}$ adjustments should be used with caution to avoid creating unfavorable reaction conditions for the amine or inactivation of EDC. EDC coupling reactions have been carried out in distilled water, ${ }^{44} \mathrm{PBS},{ }^{45}$ MES buffer, $, 7,46-48$ and borate buffer, ${ }^{49,50}$ with the $\mathrm{pH}$ maintained at $4 \cdot 5-8 \cdot 0.5,7,46-49,51$ The reaction has the best chance of succeeding if the $\mathrm{pH}$ is maintained between 4.5 and 6.5, which balances EDC stability with maintaining the reactivity of the carboxy groups. ${ }^{51}$

After identifying a solvent that will complement all of the reaction components, a systematic evaluation of reaction conditions, including time, temperature, and reactant concentrations, can be carried out. Ideally this information can be used to graphically describe the relationship between parameters like EDC, SPION, or DNA concentration and the coupling efficiency. ${ }^{7}$ EDC concentrations of 30-50 mM may be required for DNA coupling ${ }^{5,7,45}$ although the coupling efficiency will need to be balanced with minimizing 
EDC-induced particle precipitation. ${ }^{36}$ A sampling of published procedures for similar coupling setups describe EDC concentrations of $0.04 \mathrm{mM},{ }^{47} 30 \mathrm{mM},{ }^{5,45}$ and $4 \mathrm{M} .{ }^{46}$ One optimization study assessed oligonucleotide binding to carboxylic acid functionalized glass beads using 0-100 mM EDC, which revealed maximum binding at $\geq 50 \mathrm{mM} \mathrm{EDC.}{ }^{7}$ Other reports suggest that the ratio of EDC to carboxylic acid and/or amine groups may be more important than the absolute EDC concentration. ${ }^{44}$ Reported reaction times vary from 20 minutes to 18 hours. $5,7,44-47,49$ Realistically the reaction has occurred within the first 20 to 30 minutes, at which point the carboxyl groups have been activated ${ }^{51}$ and the majority of the EDC has hydrolyzed. ${ }^{13,38}$ Most protocols perform the coupling at room temperature $\left(25^{\circ} \mathrm{C}\right),{ }^{5,7,45-47,49}$ although temperatures from $0-60^{\circ} \mathrm{C}$ have been reported. ${ }^{44}$ For this specific SPION formulation, heat should be approached with caution as it causes the oleic acid surrounding the SPION to core to melt, destroying the SPIONs.

Reactions performed during the optimization procedures should be performed in at least triplicate, using SPIONs from the same batch, identical DNA sequences and concentrations, and EDC from the same container. To reduce variability due to EDC degradation during storage, EDC should either be purchased in small quantities (>1 gram) or aliquoted into single-use storage containers when the container is first opened. Reaction controls with SPIONs and DNA, but no EDC, should be included in the optimization process to control for DNA binding through non-covalent mechanisms. Additional controls include SPIONs with EDC, without DNA, and EDC with DNA, without SPIONs. 


\subsection{1c Alternative Coupling Reagent: DMTMM}

Considering the issues with EDC-mediated amide bond formation, perhaps the simplest approach to improving the reproducibility of the SPION-DNA coupling reaction is to utilize an alternative attachment method. DMTMM (4-(4,6-dimethody-1,3,5-triazin-2yl)-4-methylmorpholinium chloride) is a commercially available, easier to use alternative that overcomes many of the limitations of EDC while retaining the major advantages. ${ }^{10-}$ ${ }^{12}$ Specifically, DMTMM is stable over a wider $\mathrm{pH}$ range than EDC and has higher reaction yields. DMTMM has been used to conjugate amine-containing ligands to $P A A,,^{10,11,13}$ and is may be more efficient than EDC for doing so. ${ }^{14,15}$ Like EDC, however, high concentrations of DMTMM can also result in particle precipitation. Reported DMTMM coupling protocols are less varied than EDC-mediated procedures. DMTMM protocols are available for coupling amine-terminal ligands to PAA-coated NPs, ${ }^{10,11,13}$ which should allow for faster optimization of reaction conditions. However, minor modifications to the reagent concentrations or buffer $\mathrm{pH}$ may still be needed, as the system still needs to allow the reactants to the dissolve or suspend. ${ }^{10}$

\subsubsection{Characterization of Functionalized SPION platform}

The SPION platform is intended to be used as therapeutic, and thus it is required to have a safety profile compatible with systemic administration. That is, the underlying SPION platform should be biologically inert and therapeutic effects of the survivin ASO are expected to be tumor-specific. SPION toxicity is heavily dependent on particle 
composition and surface properties. ${ }^{19,23,52-56}$ These effects are described in greater detail in (1.2.2 Therapeutic NPs: Design Considerations). Briefly, the size and surface charge of the final SPION-ASO conjugate affect particle internalization in vitro and distribution, clearance, and half-life in vivo. ${ }^{15,23,54,57-62}$ Choice of ligand or ASO can also impact uptake, biodistribution, or biological effects. ${ }^{1,16,24,63-67}$ For ASO specifically, greater surface density enhances protein regulatory effects. ${ }^{25}$ Additionally, coatings can affect the magnetic properties of the SPION, which would in turn affect their utility as MRI contrast agents. ${ }^{32}$ Thus, obtaining reproducible toxicity results requires consistently formulated SPION conjugates..$^{30,52,55,58,59,68}$

SPIONs are prepared in a step-wise fashion, and it is important to characterize the platform at each step of the synthesis. The addition of each layer is expected to change the overall diameter, surface charge, composition, and, ultimately, biocompatibility. Thermal decomposition is used to obtain the oleic acid coated SPION core, ${ }^{69}$ which is then coated with octyl-amine modified PAA to impart aqueous solubility ${ }^{36}$ before surface functionalization. Oleic acid coated SPIONs have been thoroughly characterized, providing a baseline for comparison. ${ }^{17}$ The chosen synthesis method consistently yields SPIONs with a narrow size distribution. Although the SPION core diameter is consistently homogeneous, modifications to the procedure, like changing the ratio of oleic acid to iron pentacarbonyl, affect the mean particle size. ${ }^{69}$ Thus, the uncoated SPIONs should be regularly assessed. SPION-PAA has also been characterized, as presented in Chapter 2. However, the final, functionalized SPIONs have not yet been analyzed. Because 
SPION physical properties are dependent on the particle's composition and surface functionalization, each specific formulation will require independent analysis.

Functionalized SPIONs should be analyzed to ensure consistent formulation, to ensure that physical properties are optimized for biological use, and to allow for appropriate, reproducible dosing for further testing. Ideally the physical properties and composition of functionalized SPIONs will remain consistent within and across batches of similarly prepared particles; however, previous experience has shown that this is not always the case. Consequently, all measurements should be made on replicate samples from independently coupling reactions.

Thorough analysis of particle properties, including composition, size, charge, ligand density, will allow SPIONs from different batches to be compared (4.1.2a Characterization of Functionalized SPIONs: Size, Surface Charge, and Composition), while platform stability can be assessed by monitoring the physical properties over time (4.1.2b Characterization of Functionalized SPIONs: Environmental Interactions). Understanding of particle components will contribute to the determination, and reporting, of relevant, reproducible dose schemes. ${ }^{30}$ It will also allow for comparison across experiments and formulations, allowing the effects of various modifications to be teased out. With full characterization and detailed reporting, platforms can be compared on the basis of number of particles, iron concentration, ligand concentration, or other measures, regardless of which parameter is used to determine the platform dose. 


\subsection{2a Characterization of Functionalized SPIONs: Size, Surface Charge, and Composition}

\section{Particle Size and Surface Charge}

Size and surface charge are limiting factors in the biocompatibility, metabolism, and biodistribution of NP constructs in vivo. ${ }^{61}$ SPION synthesis via thermal decomposition results in homogeneous particle cores, ${ }^{17,69}$ but the addition of subsequent organic layers is subject to greater variability. Analysis of SPION diameter and surface charge at each step of synthesis is needed to determine the effects of each additional layer. Understanding the contribution of each layer will be critical to the presentation of this formulation as a proof-of-concept platform that can be modified with other ligands or loaded with drugs. SPION core diameter is easily assessed using transmission electron

microscopy (TEM). ${ }^{19,58}$ However, TEM does not provide information about the organic coatings, which are not expected to affect the diameter of the metallic core. To determine the diameter of the entire coated SPION system, dynamic light scattering (DLS) can be used. ${ }^{58}$ DLS measures the hydrodynamic diameter of NPs in solution, which does require that the SPIONs be stably suspended in solution. Ideally the combination of TEM and DLS will reveal changes in diameter that are consistent with the expected thickness of the coatings. Changes that are much greater than are expected may indicate particle agglomeration or issues with the coating and functionalization protocols. A narrow size distribution, in the expected diameter range, will be indicative of successful, homogeneous surface modifications. 
SPION surface charge, or z-potential, can be assessed using the DLS instrumentation. ${ }^{20,70}$ PAA-coated SPIONs need a slightly negative surface charge to remain suspended in aqueous solutions. The negative charge, arising from deprotonation of the surface carboxylic acid groups, is also required for formation of the amide bond during the coupling reaction. ${ }^{15}$ SPION-ASO is expected to have a significantly more negative surface charge due to the phosphate backbone of the nucleic acids. Under most conditions, the significant negative surface charge of the particles would be considered a hindrance to their eventual clinical use; $;^{23,61,62}$ however, it has been demonstrated the negative surface charge arising from densely packed ASO on a NP surface actually enhances particle internalization. ${ }^{25,26}$

\section{Overall Composition}

Platform composition can be analyzed in a variety of ways, all of which provide information about the composition of the entire sample being analyzed rather than the individual particles. To overcome this limitation, inter- and intra- batch replicates should be assessed. Thermogravimetric analysis (TGA) can be used to determine the contribution of each component to the total mass. ${ }^{17,32,71,72}$ We have previously found that SPION-OA is $20 \%$ oleic acid and $80 \%$ iron oxide, ${ }^{17}$ while SPION-PAA is $75 \%$ PAA, $5 \%$ oleic acid and $20 \%$ iron oxide. Analysis of composition by mass can be used to determine the number of particles in a given mass and the consistency of the formulation over time.

\section{Iron Quantification}


For SPION platforms, the iron concentration is an important factor in clinical utility ${ }^{16,29,72,73}$ and toxicity potential. ${ }^{30,57}$ While TGA can give an approximation of the iron content of a sample, as a percentage of the total mass, analytical techniques like inductively coupled plasma (ICP), ${ }^{19,61,74,75}$ atomic absorption spectroscopy (AA), ${ }^{76,77}$ and colorimetric detection methods allow quantification accurate to parts per million (ppm) or parts per billion $(\mathrm{ppb})$. Iron concentration can also be determined from magnetic relaximetry ${ }^{61,72,78,79}$ or with commercially available assay kits like the Quantichrome iron assay. ${ }^{80}$

ICP is the most sensitive iron quantification method, with limits of detection for iron in the ppb to ppt range. ${ }^{19} \mathrm{ICP}$ also has a detection range over several orders of magnitude, which is helpful when the solution concentration is entirely unknown. An alternative to ICP is $A A$, which is mechanistically similar to ICP but requires slightly higher concentrations of analyte. AA can only be used to quantify a single element per sample/run while ICP can quantify a number of elements simultaneously. A third method of quantifying iron, which can be performed with more commonly available equipment, is the use of an indicator dye that changes color upon reacting with iron. The resulting color change can be quantified with UV-vis spectrometers, including the forms commonly found in biological labs, like a plate reader or Nano Drop. A number of colorimetric iron indicators have been described, including Prussian blue,,$^{81,82}$ ferrozine, ${ }^{83-85}$ and o-phenanthroline. The major limitation of colorimetric methods is that they are expected to have a much more limited 
linear range of detection than ICP or AA, meaning it could require several trials to dilute the SPION sample of unknown starting concentration to the appropriate range.

One unfortunate limitation of all the iron quantification techniques mentioned is that they detect soluble iron, which requires acid digestion of the sample to fully dissolve all particulates. The sample is then diluted for analysis, and the iron concentration of the final, diluted sample must be within the detection limits of the instrument being employed. When synthesis and coupling steps are carried out on a small scale, as they are during optimization studies, iron analysis can destroy most or all of the prepared SPIONs. However, with consistently formulated SPIONs, iron content could be correlated to a less intrusive measure, such as ligand concentration, rather than carrying out iron analysis on each batch.

\section{Ligand (ASO) Quantification}

This SPION platform is functionalized with a survivin ASO, ${ }^{86}$ a ligand chosen for the specificity of its therapeutic effects. Quantifying the SPION-bound ASO is a critical step characterization, as it will allow doses to be calculated based on the therapeutic ligand. Then, the degree to which a given concentration of ASO alters protein expression when introduced as SPION-bound, free, and through traditional transfection methods can be compared. ASO are expected to have time- and concentration dependent effects on protein expression ${ }^{87}$ and comparing the efficacy our formulation to existing ASO technologies will require a measure of equivalent exposure. Additionally, ligand density 
on the SPION surface may impact biological effects, even at the same ASO concentrations. When gold nanoparticles are similarly loaded with ASO, greater surface density of the ASO resulted in stronger protein regulatory effects in vitro. ${ }^{25,26}$ The concentration of SPION-bound ASO can be determined indirectly by collecting unbound from the coupling reaction. ${ }^{7}$ SPION-ASO conjugates can also be directly evaluated via UV-vis ${ }^{88}$ or gel electrophoresis. ${ }^{45,89}$ Incorporation of fluorescently labeled ASO can be quantified using flow cytometry or a fluorimeter. ${ }^{90,91}$

\subsection{2b Characterization of Functionalized SPIONs: Environmental Interactions}

\section{Interactions with Plasma Proteins}

NPs interact with biological molecules and salts in media and storage solutions. ${ }^{26,58,60,61}$ Although these interactions are primarily electrostatic, as opposed to covalent, they can still have a significant impact on the particle size, shape, and surface charge. Protein adsorption, then, can have a direct and drastic impact on NP uptake in vitro and distribution and clearance in vivo. ${ }^{21} \mathrm{NP}$ surface chemistry affects particle interactions with plasma proteins. ${ }^{26,58}$ Negatively charged NPs are more likely to interact with proteins that have specialized cellular uptake mechanisms. ${ }^{60,61}$ With spherical nucleic acids, increased ASO density on the particle surface increased interactions with plasma proteins, resulting in a more positive (but still negative) zeta potential and leading to increased uptake. ${ }^{26}$ In vivo, many NPs are rapidly coated with opsinins, or proteins that recruit macrophages to initiate clearance of the foreign material. ${ }^{15,23,54,58,59}$ Inert, 
biocompatible surface coatings like poly(ethylene glycol) (PEG), dextrans, or synthetic block co-polymers increases circulation half-life by reducing plasma protein interactions and, thus, minimizing clearance by macrophages. ${ }^{15,16,23,29,92}$ However, nucleic acids, including the ASO used in our platform, have a strong negative charge and are expected to interact with plasma proteins. ${ }^{26}$ In addition to directing SPION uptake and clearance, plasma protein interactions can have the unintended, and often unaccounted for, consequence of binding to the soluble nutrients in in vitro setups and effectively starving the cells. ${ }^{20}$

To account for the interaction of SPIONs with biological media, the physical properties of SPION-ASO should be re-characterized after incubation with cell culture media or plasma and these properties should be reported with the properties of the platform prior to the incubation. ${ }^{58}$ If further analysis is desired, protein-particle interactions can be quantified and adsorbed proteins can separated and identified using gel electrophoresis and mass spectrometry. ${ }^{70}$ SPIONs can be incubated with cell-free media prior to in vitro experiments to avoid unintentionally inducing toxicity through nutrient depletion. ${ }^{20,90}$

\section{Solution stability}

SPION size, surface charge, and composition will be evaluated after several days, several weeks, and several months in the storage solution. Changes in particle size, due to particle agglomeration or interaction with solution components, will impact biocompatibility and cellular uptake.$^{93}$ Solution $\mathrm{pH}$ can affect surface charge, as can 
interactions with salts in the storage solution that effectively screen surface charges. ${ }^{21,93}$ Composition will be assessed to ensure that the particle coating, ligand binding, and, if relevant, drug loading, remain stable.

\subsubsection{Evaluation of Biological Effects of SPION-ASO}

In order for SPION-ASO to progress towards eventual clinical use, biocompatibility and therapeutic efficacy of the SPION-ASO platform must be demonstrated both in vitro and in vivo. The biological effects will first be assessed in vitro (4.1.3a Evaluation of the Toxicity of SPION-ASO In Vitro), where the specificity of the therapeutic effects can be validated. The SPION platform is expected to be inherently non-toxic, and functionalization with the survivin ASO should limit toxicity to survivin expressing cells. Cells with varied basal survivin expression will be exposed to SPION-ASO and toxicity will measured through multiple endpoints, including particle uptake, changes in metabolic viability, apoptosis, and sensitization to chemotherapy drugs. In vivo murine and (rat) models will then be employed to assess biodistribution, clearance, systemic toxicity, MRI contrast enhancement, and anti-tumor effects (4.1.3b Evaluation of the Toxicity of SPIONASO In Vivo).

\subsection{3a Evaluation of the Toxicity of SPION-ASO In Vitro}

\section{Appropriate Cell Lines}


The survivin ASO was chosen to impact survivin expressing cancer cells without impacting healthy cells. Thus, the aim of the in vitro studies is to demonstrate specificity of therapeutic action. The first step of the in vitro evaluation involves demonstrating that SPION-ASO has a measurable effect on a survivin-expressing cell line. Controls for these studies should include SPIONs conjugated to a non-targeted DNA sequence of equivalent length (NTC). Preliminary studies were carried out on A549 lung adenocarcinoma cells. A549 cells have been used for many previous studies on the effects of regulating survivin expression in vitro, ${ }^{86,94-97}$ which serves to both validate the choice of cell line and to provide resources for comparing results.

Once effects therapeutic effects have been demonstrated, the specificity of the effects can be evaluated in cell lines with varied basal survivin expression. ${ }^{95}$ ASO targeting survivin have previously been found to have little to no effects on cell lines that do not express survivin; 86,98 however, validating this specificity will also provide further evidence that the SPION-ASO construct is not inherently toxic. Survivin expression has been quantified in many commonly used cancer cell lines. ${ }^{94,95}$ Differences in survivin expression between parental and modified, including drug resistant or transformed, cells of the same origin would also provide interesting comparisons on the effects of basal survivin expression level.

\section{SPION Uptake and Intracellular Localization}


Monitoring of SPION uptake and intracellular localization will provide insight into the mechanisms of therapeutic effect and, if relevant, toxicity. SPION uptake can be tracked both qualitatively and quantitatively. Qualitatively, TEM can be used to visualize the locations of the SPION cores relative to intracellular structures. ${ }^{19}$ Detailed intracellular images can be obtained with TEM, although laborious sample preparation is required for visualization of biological samples. TEM is most valuable for viewing metals, like the SPION core, and does not provide information on the state of the particles coatings or ligands. Thus TEM images of complex SPION platforms should be used in conjunction with other techniques to draw conclusions about particle intracellular localization. Alternatively, the ASO can be fluorescently tagged prior to conjugation with the SPION and uptake can be tracked with fluorescent microscopy. ${ }^{58}$ While this technique is commonly used, there is a possibility that the fluorescent dyes will change the uptake and distribution patterns by altering particle size, surface charge, and plasma protein interactions. However, tracking SPION uptake using fluorescent microscopy can complement TEM localization data. While TEM is reliable for locating the SPION core, fluorescent microscopy is only able to convey the location of the tracer dye, which in our setup is covalently linked to the ASO ligand. If fluorescent images conflict with TEM images, the possibility that the ASO has been cleaved from the SPION core or the dye has been cleaved from the ASO should be considered.

Particle uptake can be determine quantitatively using measures on intracellular iron. The analytical techniques are the same as those used to determine the iron concentration during particle characterization, which is discussed in greater detail in 4.1.2a 
Characterization of Functionalized SPIONs: Size, Surface Charge, and Composition. However, modifications to the procedures may be needed to remove cellular debris. It is also important to note that the iron quantification techniques measure all soluble iron, including iron that was present in the cell before treatment. Thus, untreated controls are needed to determine the baseline iron concentration the cells. Additionally, these iron quantification methods require acid digestion of the entire sample prior to analysis. As a result, they do not provide any information about intracellular particle degradation prior to analysis.

\section{Changes in Cell Behavior}

Changes in cellular behavior and function will give further insight into the biocompatibility and mechanisms of action of ASO-SPIONs. Viability assays are an efficient way to rapid assess a range of doses and time points. The results can then be used to direct the dosing and time points for additional studies. Cell viability can be quantified colorimetrically by incubating cells with tetrazolium reagents like MTT (3-(4,5dimethylthiazol-2-yl)-2,5-diphenyl tetrazolium bromide), MTS (Cell Titer 96 Aqueous One Solution Cell Proliferation Assay from Promega, or WST-1. ${ }^{19,30,58}$ Mitochondrial enzymes convert the reagent to a formazan dye, resulting in a color change proportional to the number of metabolically active cells. SPION-ASO is expected to reduce the viability of survivin expressing cells but should not alter the viability of non-survivin expressing cells. SPION-NTC is not expected to affect the viability of any cell line, which will confirm that the platform is not inherently toxic. In preliminary studies, none of the SPION-NTC doses 
tested decreased cell viability. Changes in viability should be assessed, at a minimum, after 24,48 , and 72 hour exposures. ${ }^{86}$ In our preliminary studies, changes in viability were not apparent following the 24 hour exposure. By 48 hours, however, significant changes were noted. One measure of the therapeutic efficacy of the SPION-ASO will be the cell viability following a 72 hour exposure, which can be compared to the effects of our specific ASO alone. ${ }^{86}$

Decreased cell membrane integrity, measured by lactate dehydrogenase (LDH) release, can serve as an indicator of cell death. ${ }^{19,30} \mathrm{LDH}$ is in an intracellular enzyme that is released when the cell membrane integrity is compromised, as it is in necrosis and the late stages of apoptosis. Like viability assessments, $\mathrm{LDH}$ is a rapid, colorimetric assay that can be used to screen a variety of conditions. However, LDH has a half-life of 9 hours once released from the cells and assays must be carefully timed to capture LDH within this window. ${ }^{99}$ Improperly timed assays of treatments that cause rapid cell lysis, and thus fewer viable cells will detect less LDH in the treated cells. ${ }^{100}$ Examination of cells prior to conducting the assay will help validate results, as will comparing the LDH results to the number of viable cells determined by MTT, MTS, or WST-1.

Cell viability assays function as a rapid indicator of changes in cell proliferation, but changes in viability alone do not give a complete picture of toxic effects. The combination of viability assays and an indicator of cell death, like LDH release, allows for comparison of live, metabolically active versus dead cell populations. Although that is an improvement 
over either measure alone, cells that are in intermediate states, like those that are in the process of dying, are still not captured.

Apoptosis is the process of orderly cell death. The cell membrane remains intact until late in the process, meaning that the cell will not contribute LDH during early stages despite the cells inevitable demise. Induction of cell death through apoptosis, as opposed to necrosis, is particularly relevant to our SPIONs, as the SPION-ASO platform was designed for eventual clinical use. In addition, the target protein, survivin, is anti-apoptotic and decreases in survivin expression, as would be seen with SPION-ASO, are expected to induce apoptosis. Apoptosis can be assessed by Hoescht staining, flow cytometry (FACS), or caspase activation assays. ${ }^{19,58,93,101}$

\section{Survivin ASO Specific Effects}

Ligand specific effects should also be monitored. With the SPION-ASO platform, changes in survivin expression are the intended mechanism of therapeutic benefit. Downstream effects of the decrease in survivin expression, including changes in cell cycle distribution, proliferation, and sensitivity to chemotherapeutic drugs and other apoptotic stimuli, are also quantifiable.

Changes in survivin expression can be directly assessed via Western blots. With ASO introduced through transfection, changes in survivin expression are notable at 24 hours and progress until at least 48 hours post-treatment. ${ }^{102}$ Changes in survivin expression should not occur when cells are treated with SPION-NTC. 
Changes in cell cycle distribution are expected when survivin is overexpressed or inhibits. Survivin overexpression is correlated with increased proliferation rates, while survivin inhibition has been linked with cell cycle disruptions. The increased proliferation rates were associated with accelerated S phase and resistance to G1 arrest. ${ }^{101}$ Survivin inhibition, and the resulting increase in apoptosis, were associated with G2/M phase arrest. ${ }^{101,103}$ Cell cycle distribution can be assessed using flow cytometry. ${ }^{104,105}$

Survivin expression is correlated with chemotherapy and radiation resistance in vitro and in vivo. ${ }^{86,98,103,106-116}$ When survivin expression is knocked out, with ASO or small molecule inhibitors, these effects are reversed. The ability of SPION-ASO to sensitize cancer cells to treatments will contribute to improvements in quality of life during chemotherapy treatment, as lower systemic doses will be needed to kill sensitized cells. SPIONs can also be loaded with chemotherapeutic agents, like doxorubicin or cisplatin, allowing for simultaneous sensitization and treatment. ${ }^{34,46,117-121}$ Cell viability, assessed with WST-1 or MTS, can be used to demonstrate sensitization.

To best demonstrate the tumor sensitization potential, the timing of ASO-induced survivin downregulation will need to be considered when establishing a treatment schedule. The strongest sensitization effects are most likely to be noted while survivin expression is at a minimum, which may require SPIONs to be added 24 to 48 hours before the cells are treated with the chemotherapy drug. Survivin inhibition has been shown to increase cell sensitivity to cisplatin, $86,106,122$ gemcitabine, ${ }^{107}$ taxols, ${ }^{123}$ and tamoxifen. ${ }^{124}$ 
Cisplatin sensitivity would be a good starting measure, as cisplatin resistant A549 cells are readily available and the results could be easily compared to the initial toxicity studies. Previous reports show significant differences in cell proliferation between the parental and cisplatin-resistant lines of both $\mathrm{A} 549$ and $\mathrm{H} 460$ at cisplatin concentrations from $0.1 \mu \mathrm{M}$ to $100 \mu \mathrm{M}^{125}$

\section{Mechanisms of Toxicity}

SPION-ASO is not expected to be associated with significant toxicity to non-survivin expressing cells, and the toxicity in survivin expressing cells should be a consequence of the ASO rather than inherent SPION toxicity. If toxicity is discovered, efforts should be redirected to reformulating the platform. However, demonstrating an understanding NP toxicity, and the mechanisms of that toxicity, may be required to move NP-based therapeutics forward in the regulatory process. A major concern for general NP toxicity, as well SPIONs specifically, is the generation of reactive oxygen species (ROS). ${ }^{19,30,58}$ ROS are most likely to be generated due to the reactivity of the SPION core, ${ }^{52,53,59}$ a risk that is mitigated in this case by the thick polymer coating. ${ }^{15,29-35}$ Once present, ROS are themselves highly reactive and can cause damage to DNA, proteins, lipids, and nucleic acids. Electron paramagnetic resonance (EPR) and fluorescent assays can be used to detect ROS. NP-induced genotoxicity, due to ROS or direct interaction of NPs with genetic material, is also possible..$^{19,30,58}$ Flow cytometry and comet assays can be used to assess physical DNA damage, while microarrays can offer insight into changes in gene regulation. 


\subsection{3b Evaluation of the Toxicity of SPION-ASO In Vivo}

After demonstrating the biocompatibility of SPION-ASO in vitro, the safety of the platform must be evaluated in vivo. SPION-ASO is intended to be safe for systemic intravenous administration. Systemic administration will theoretically allow the SPIONs to localize to tumors and metastases due to the enhanced permeability and retention effect. $^{29,57,126}$ However, local delivery, like direct injection into a known tumor, is also a possibility. Initial in vivo studies will focus on monitoring SPION-ASO biodistribution and systemic adverse reactions. Therapeutic endpoints will include tumor growth inhibition and tumor survivin expression. SPION-related MRI contrast enhancement will also be assessed.

Biodistribution will be determined from tumor and organ analysis. Organ and tumor iron content will be quantified using ICP. ${ }^{19,127}$ Tissue sections will be stained for iron, using Prussian blue, to visualize tissue distribution. ${ }^{19,76,93,128}$ The contrast-enhancing effects of SPIONs will also allow their distribution to be visualized with MRI. ${ }^{76,128}$ Systemic toxicity will be evaluated by monitoring changes in organism behavior, weight, appearance, and blood chemistry. Induction of systemic inflammatory or immune responses would increase plasma levels of IL-1 $\beta$, IL- 6 and TNF- $\alpha$ cytokines. ${ }^{93}$ SPION interactions with the liver and kidneys as a result of clearance and physiologic filtration could cause target organ damage. Serial blood levels of serum proteins that indicate hepatic or renal 
damage, including alanine aminotransferase, aspartate aminotransferase, bilirubin, creatinine, albumin, and total protein will be used to monitor for target organ damage. ${ }^{19}$

\subsubsection{Selection of a Targeting Ligand to Improve the Selectivity of SPION Uptake by Survivin-Expressing Cancer Cells}

A well-designed SPION system can serve as both a drug delivery vehicle and an MRI contrast agent. SPION conjugation with an ASO targeting a protein primarily found in cancer cells, like survivin, allows for specificity of therapeutic action. While specificity of action will reduce unintended adverse effects, SPION-ASO conjugates have an intracellular target and thus do not contribute to tumor-specific particle distribution or uptake. A ligand directing improved specificity of uptake by target cells would allow the platform to be used as an MRI contrast agent and/or a delivery vector for high concentrations of cytotoxic drugs. Previously, SPION conjugation with aptamers, ${ }^{27}$ antibodies, ${ }^{15,27,28,49}$ and biomolecules like folate ${ }^{34,66,84,129-132}$ has been shown to increase the specificity of accumulation in vivo. Thus, identification of a target specific to survivinexpressing malignant cells would enhance the utility of the SPION system described in Chapter 2.

One potential target is the unique manner in which many cancer cells process survivin. Survivin in cancer patients, but not healthy people, is digested and select fragments are then displayed on the cell surface as part of the major histocompatibility type I complex $(\mathrm{MHC})$, particularly on human leukocyte antigen A (HLA-A), through a pathway that 
typically handles foreign proteins. ${ }^{133}$ There are several HLA-A subtypes, and there is evidence that HLA-A subtype is the defining factor in the survivin epitope displayed, independent of the cancer type. ${ }^{134}$ The viability of this complex as a therapeutic target was demonstrated with the development of a vaccine that sensitized patient T-cells to a survivin epitope. ${ }^{116}$ Thus, a ligand that could possibly be engineered to be selective for this survivin-HLA complex, which is unique to cancer cells, could be conjugated to SPIONs to increase the selectivity of SPION uptake in vivo.

Aptamer selection is a time- and labor-intensive process. Although our intended target is well described, it is not known if an aptamer can provide the specificity needed to recognize the survivin nonapeptide bound to HLA. To streamline the selection process, in silico modeling will be employed as a first step (4.1.4a In Silico selection of an aptamer targeting survivin-expressing cells). Starting with in silico modeling will provide a better understanding of the possibilities of targeting peptide bound HLA, and will allow for prediction of cross-reactivity between the survivin peptide fragments displayed on different HLA isoforms. The modeling process will, ideally, identify aptamer sequences that are expected to bind the target with high affinity and specificity. If the aptamer modeling fails to identify potential aptamer sequences, aptamer selection can be carried out in vitro using either isolated MHC-survivin epitope complexes ${ }^{134}$ or whole-cell SELEX ${ }^{135}$ (4.1.4b Selection of an aptamer targeting external feature of survivin expressing cells).

\subsection{4a In Silico selection of an aptamer targeting survivin-expressing cells}


The basic approach will be align published peptide sequences (survivin derived) with the crystal structure of a peptide bound to HLA (here called the reference nonapeptide). This will provide a composite from which the predicted binding elements involved will be identified, based on the known binding of the reference nonapeptide. These peptides will then be docked to a library of aptamers and the result rank-ordered. Selection of the best peptide-aptamer complexes (best will be defined) will be further examined. In particular, high scoring complexes that utilize the predicted region for binding to the aptamer will be selected. The aptamer and peptide comprising this complex will be made and their binding measured by surface plasmon resonance (SPR). The aptamer yielding the highest affinity by SPR will then be attached to SPIONs that also bear antisense survivin, and the resulting SPIONs will be assayed for binding to survivin expressing and nonexpressing cells.

The peptides that will be used come from Bachinsky, et.al. ${ }^{136}$ and Reker, et.al. ${ }^{133}$ The binding affinity of each peptide to HLA-A is known and combining these two data sets will provide approximately 100 peptides. Each nonapeptide will be built and energy minimized using Amber. ${ }^{137}$ Since nonapeptides are too short for accurate structure prediction, we will use simulated annealing to generate multiple conformations. Only the conformation with the lowest root-mean-squared deviation (RMSd) to the reference nonapeptide will be retained. Once all nonapeptides are aligned with the reference peptide, the predicted binding regions of each peptide will be determined by comparison to the known binding regions of the reference nonapeptide. All peptides will then be screen against a library of aptamer candidates ${ }^{138}$ using AutoDock. ${ }^{139}$ Chushak has provided us with this database. 
The resulting set of peptide-aptamer complexes will be rank-ordered based on the docking score. The top 100 complexes will be examined to determine the binding mode of the peptide and those that do not use the binding motif predicted from their alignment with the reference nonapeptide will be discarded. Further, the aptamer component of these complexes will screened in silico peptides that are known to bind poorly or not at all to HLA-A and those ranked high will also be removed with this process simulate negative selection. The peptides that remain from this process will be examined for their affinity for HLA-A complexes containing common viral peptides.

Surface Plasmon Resonance (SPR) measurements of relative binding affinities will be made on a Biacore X1000. ${ }^{140}$ Gold films on glass will be treated in an ethanolic solution of mercaptoundecanoic acid (MUA) and octane thiol (1:3), placed in the sample holder and activated with NHS/EDC followed by exposure to the peptide target (purchased from Peptide 2.0, Chantilly, VA) under examination (one selected from a nonapeptide-aptamer complex predicted from the in silico studies). This will result in an amide bond between the N-terminus of the peptide and the MUA. For peptides containing non-terminal lysines, mecaptoundecanoaimine will be used in place of MUA and the C-terminus of the peptide will be activated with NHS/EDC and bonded to the SAM. The aptamer partner will then be flowed over the chip and the binding constants determined by measuring the SPR response over time (i.e., during association and dissociation phases) as a function of aptamer concentration. 


\subsection{4b Selection of an aptamer targeting external feature of survivin expressing cells}

If potential aptamer characteristics are identified with in silico modeling, sequences with the identified features will be used as the starting point for our library for in vitro selection against an isolated recombinant target. E. coli will be modified to produce human HLA-A, which will then be mixed with survivin peptide fragments to generate the isolated protein target. ${ }^{134}$ Negative selection will be carried out against HLA-A bearing common viral proteins.

Although the survivin peptide - HLA-A complex has been isolated, it is possible that we will be unable to replicate this procedure. If this becomes a problem, we will proceed with whole cell SELEX, ${ }^{135}$ beginning with a library based off our most likely aptamer sequences from modeling. In the event that whole cell selection is required, HLA-type would be used to match survivin expressing cell lines with appropriate survivin-deficient cell lines for negative selection.

After several rounds of both positive and negative aptamer selection, we anticipate identifying and sequencing an aptamer that binds selectively to our target. Although the aptamers are expected to be relatively HLA-type specific, an aptamer that binds to more than one type might actually be advantageous in vivo since even small mutations can impede aptamer binding and cancer cells are known for their high mutation rates. This aptamer can then be coupled to the SPION platform to allow for specific targeting of survivin expressing cancer cells. 


\subsection{Role of MSLN in CNT-induced Malignant Transformation}

Malignant transformation takes place over a protracted period of time, during which genetic damage accumulates. ${ }^{141}$ Although cancer development occurs as more of a continuum than as a series of discrete steps, it is usually difficult to identify and study the early stages of the transformation period. Consequently, most cancer studies are conducted using definitively malignant cells. Even for particle-induced malignancies, there can be several decades between the inciting exposure and clinical detection. Methods allowing for the identification and investigation of early genetic and behavioral changes would offer greater mechanistic insight than can be obtained from the current binary classification of malignant or non-malignant.

An in vitro chronic exposure model has been developed to study the effects of chronic (6 month) exposures to sub-acute doses of SWCNT, ${ }^{142-144}$ MWCNT, ${ }^{142,144}$ asbestos, ${ }^{142,144}$ and metals. ${ }^{145}$ Using this model, the transformative effects of chronic CNT exposures have been demonstrated in non-cancerous bronchial epithelial cells (BEAS$2 \mathrm{~B}^{143,146,147}$ and SAEC ${ }^{142}$ ) as well as in a mesothelioma line (Met5A). ${ }^{144}$ So far this exposure model has been used to study the transformed cells, but, moving forward, this chronic exposure model could be used to better understand the dynamic transformation process. Studying cell populations from numerous time points throughout the 6 month exposure would help map out the order in which changes occur, and would allow for comparison of the damages from different materials. Obviously studying all possible 
changes would be inefficient. A more streamlined approach would be to look at specific pathways, mediators, or expected changes.

Specifically, using this chronic transformation model, subtle changes in cellular morphology, behavior, and function can be assessed over time to better understand the course of cancer development. Using our previously generated, CNT-transformed bronchial epithelial cell line (BSW), we have begun to look at the functional role of MSLN in the malignant phenotype (Chapter 3). After determining that MSLN was overexpressed in the BSW cells, a stable MSLN knockdown line (BSW shMSLN) was generated. We found that while BSW cells were more aggressive than their untransformed counterparts, knocking down MSLN reverted BSW shMSLN to more normal behavior. To better understand the mechanism by which MSLN was contributing to the malignant behavior, IPA was used to identify potential upstream mediators and downstream effects of mesothelin overexpression in CNT-transformed bronchial epithelial cells (BSW). Many likely relationships were identified, including mediators involved in cell proliferation and cell cycle regulation, apoptosis, and metastasis. We previously confirmed MSLN-related changes in cyclin E expression; however, the remaining relationships have not been validated (4.2.1 MSLN-Related Changes in Gene Expression). A potential relationship between MSLN and the anti-apoptotic mediator survivin has also been identified (4.2.2 MSLN as a Survivin Regulator).

\subsubsection{MSLN-Related Changes in Gene Expression of CNT-transformed Cells}


We demonstrated the functional contribution of MSLN to the development of malignant phenotypes following chronic CNT exposure (Chapter 3); however, the mechanisms behind those changes are still largely unaccounted for. The mechanisms by which MSLN contribute to carcinogenesis and tumor aggression, in general, are not well understood. ${ }^{148,149}$ Predictions as to the molecular mechanisms driving the CNT-induced carcinogenesis were generated to focus our mechanistic studies. Gene expression changes following chronic CNT-exposure of bronchial epithelial cells were analyzed using microarrays. ${ }^{143,150}$ Gene expression in CNT-transformed cells was compared to unexposed controls to identify the pathways that were most affected. IPA was then used to predict the effects of knocking out MSLN expression in the transformed BSW cells (Chapter 3). Although the predicted effects are in line with our observed changes in cell behavior following MSLN knockdown, the majority of the IPA predictions have not yet been verified in our transformed cells. MSLN-related changes in gene expression will be investigated (4.2.1a Validation of Predicted MSLN-induced Changes in Protein Expression). Upstream changes expected to contribute to MSLN overexpression include increase in TNF- $\alpha$, K-ras, and decreases in cyclin A, cyclin B, and CDK1. MSLN overexpression then induces, directly or indirectly, IL-6, Stat3, cyclin E, MMP-9, and MMP-7, while inhibiting p53 and the pro-apoptotic mediator BAX. MSLN and other relevant mediators will then be quantified in cell samples collected from various time points through the transformation process, with the aim of determining a time line of the changes (4.2.1b Evolution of Gene Expression During CNT-induced Transformation). Changes in gene and/or protein expression will be correlated with cell behaviors. At the 
conclusion of these studies, significant insight into the mechanistic role of MSLN in the development of MSLN-related aggressive behaviors will have been gained.

\subsection{1a Validation of Predicted MSLN-induced Changes in Protein Expression}

Our previous MSLN studies were carried out with CNT-transformed bronchial epithelial cells (BSW) and a stably transfected BSW MSLN knockdown line (BSW shMSLN) has already been generated. IPA predictions of MSLN interactions were specific to this cell line, although many of the relationships are expected to be generalizable as they have been shown previously in other cancer cell lines. Western blots will be used as a first step to identify significant changes in protein expression between BSW, BSW shMSLN, and passage matched BEAS-2B controls. Expected changes include increased TNF- $\alpha$, K-ras IL-6, Stat3, cyclin E, MMP-9, and MMP-7, and decreased cyclin A, cyclin B, CDK1, p53, and BAX. Changes in protein expression detected by Western blot will be correlated with the appropriate cell behaviors. The altered protein expression profile is expected to be readily detectable, as MSLN overexpression has previously been shown to induce cyclin E, ${ }^{151}$ MMP-7, ${ }^{152}$ MMP-9,,${ }^{152,153}$ IL-6, ${ }^{149,151,154}$ and Stat3 ${ }^{151}$ and to inhibit p53 ${ }^{155}$ and BAX. ${ }^{155}$ Additionally, decreases in p53 are known to have an important role in CNT-induced cell cycle dysfunction. ${ }^{143,156}$ To give a more complete picture of MSLN effects, BSW shMSLN gene and protein expression will be assessed through DNA microarray analysis and an apoptosis protein array, comparable to the ones used on the BSW cells previously. ${ }^{150}$ 
The role of MSLN overexpression in the transformations observed in our other chronic exposure models has not been studied. However, similar post-exposure effects, including increased proliferation, increased invasion and migration, and apoptosis resistance have been noted following a variety of chronic in vitro NP exposures. ${ }^{142,144,145}$ To use these cell lines, MSLN expression in the transformed and passage matched controls would first need to be quantified. MSLN overexpression in these lines, including a second variety of non-cancerous lung epithelial cells, ${ }^{142,144}$ would allow for further validation of the role of MSLN in CNT-induced carcinogenic transformation. A mesothelioma line, Met5A, has also been used to model chronic transformation. ${ }^{144}$ Mesotheliomas, including Met5A cells, highly express MSLN at baseline. Comparison of the MSLN- and whole-genome related changes in transformed Met5A cells versus non-cancerous lung epithelial cells would offer insight into the role of MSLN in the transformation process. Thorough analysis of the genomic and protein expression changes in multiple cell lines following exposure to CNT through the same model can be used to identify recurrent patterns in the molecular mechanisms of chronic CNT-induced damage.

\subsection{1b Evolution of Gene Expression During CNT-induced Transformation}

Validation of MSLN-related changes in CNT-transformed cells offers a starting point for understanding the transformation process. Ultimately, however, the best way to understand the transformation is to study the cells as they transition from the noncancerous parental line to the aggressive and tumorigenic state. Presumably sample populations of CNT-exposed and passage-matched controls were frozen down 
throughout the 6 month exposure. These stored cells can be used to document the changes in cell morphology, behavior, and gene expression, allowing a step-by-step reconstruction of the transformation process. Cells from the transition period may further change if they are passaged too many times, so thawed cells should be used quickly and passage number should be meticulously documented to minimize the impact of these effects. Limitations on handling these cells may preclude longer experiments but are not expected to impede short-term studies.

Expression of MSLN and other critical mediators, including those discussed in the previous section, should be quantified in the transition-phase cells. Based on the IPA predictions, MLSN overexpression is induced at least in part by increases in TNF- $\alpha$ and K-ras, and the increases in either or both before MSLN levels rise would lend support to that prediction. There is also a chance that MSLN is involved in a positive feedback loop where TNF- $\alpha$ induces MSLN, which induces IL-6, and then IL-6 further induces TNF- $\alpha$. Cell proliferation rate can be used as a fast and simple screening to identify the onset of cell cycle dysfunction. Similar setups can be used to determine apoptosis resistance. ${ }^{143,147}$

\subsubsection{MSLN as a Survivin Regulator}

Tumor overexpression of both MSLN $148,149,157,158,152$ and survivin $86,103,106,109-116$ is, independently, correlated with more aggressive phenotypes and overall worse outcomes. The presence of either protein is strongly associated with more rapid cell proliferation, 
apoptosis resistance, and increased invasion and migration in vitro and metastases in vivo. While survivin has been hot topic in cancer research for over a decade, MSLN has not been as well described. Although on the surface MSLN and survivin seem to have little in common, there is significant overlap between the consequences and proposed molecular pathways driving the consequences of their upregulation. Specifically, MSLN constitutively activates NF-k $\beta$, increasing the production of IL-6, which increases Stat3 expression. ${ }^{149}$ Stat3 is an upstream regulator of various anti-apoptotic mediators, including survivin. As Stat3 binds acts as a transcription factor for survivin, by binding directly to the survivin promoter, ${ }^{159,160}$ increases/decreases in Stat3 expression have been linked to increases/decreases, respectively in survivin expression. ${ }^{161,162}$ It seems reasonable, then, that decreasing MSLN expression would indirectly result in decreased survivin expression. Identification of a relationship between MSLN and survivin would significantly enhance the understanding of MSLN downstream effects.

Although MSLN is probably not the sole inducer of survivin in real-world settings, it is very likely that is plays an important role in MSLN-related aggressive behaviors and that this relationship can be detected in vitro. To determine if MSLN overexpression can upregulate survivin, appropriate cell lines must first be chosen. To do so, MSLN and survivin expression of a variety of cell lines will be quantified (4.2.2a Identification of MSLN and Survivin Co-Expressing Cell Lines). Then stable MSLN knockdowns will be generated and used to investigate the resultant changes in survivin expression as well as changes in cell behavior and expression of target genes (4.2.2b MSLN Knockdown is Expected to Decrease Survivin Expression and Mitigate Malignant Phenotype). Last, 
survivin expression in MSLN-knockdown cells will be induced and changes in cell behavior and target gene expression will again be assessed (Overexpression of both survivin and MSLN is known to increase MMPs, 152,164,153 cyclin $E,{ }^{101,160,151}$ and $\mathrm{Bcl}-$ $2.149,165,166$ Repression of p53 is also expected. ${ }^{108,143,160,167}$

\subsection{2c Induced Survivin Expression Restores Apoptosis Resistance, Cell Cycle} Dysfunction, and Invasiveness. Restoring survivin expression independent of MSLN will aid in identifying any survivin-related changes that are induced by MSLN overexpression.

\subsection{2a Identification of MSLN and Survivin Co-Expressing Cell Lines}

Cell lines with high levels of both MSLN and survivin are expected to have the greatest potential for demonstrating MSLN-related changes in survivin expression. MSLN and survivin co-expression will be quantified in a number of cell lines and human tumor lysates. The easiest starting point for this step would be to use cell lines that are already known to have high MSLN expression, including BSW, mesothelioma, ${ }^{163}$ or pancreatic cancer ${ }^{154}$ cell lines. Transformed cell lines generated using our in vitro chronic exposure model ${ }^{142-145}$ are also likely to have increased MSLN expression. Analysis of cell populations from time points throughout the exposure and transformation process would provide a timeline and progression of the changes. Additionally, analysis of human tumor lysates will ensure that the studies are providing clinically relevant insight. 


\subsection{2b MSLN Knockdown is Expected to Decrease Survivin Expression and Mitigate Malignant Phenotype}

MSLN and survivin co-expressing cell lines, identified in the previous step, will be used to study the role of MSLN in survivin overexpression. Stable MSLN knockout lines (shMSLN) will be generated using shRNA. MSLN expression in the knockout lines will be assessed and lines with the least MSLN will be selected for initial analysis. However, if MSLN knockdown is found to have an effect on survivin expression than the dosedependence of that effect can be quantified ${ }^{164}$ using cell lines with intermediate levels of MSLN expression. Evaluation of the aggressiveness of the shMSLN lines can then be

assessed in terms of apoptosis resistance, ${ }^{143}$ cell cycle dysfunction, and invasion and migration. The effects of MSLN knockdown, and subsequent changes in survivin expression, on drug sensitivity and tumor formation in vivo can also be investigated. Microarrays will be used to identify changes in gene expression. Overexpression of both survivin and MSLN is known to increase MMPs, ${ }^{152,164,153}$ cyclin $\mathrm{E},{ }^{101,160,151}$ and $\mathrm{Bcl}-$ 2. ${ }^{149,165,166}$ Repression of p53 is also expected. ${ }^{108,143,160,167}$

\subsection{2c Induced Survivin Expression Restores Apoptosis Resistance, Cell Cycle Dysfunction, and Invasiveness}

Survivin expression will then be rescued in the shMSLN cells. ${ }^{168}$ Survivin rescue, while still inhibiting MSLN, will demonstrate which pathways are downstream of survivin versus those effects that are unique to MSLN. Cell behaviors and changes in gene expression 
will be assessed as described in the previous section. Strong correlations between survivin expression and tumor aggressiveness have been documented in across nearly all types of cancer, so survivin rescue is expected to restore these behaviors. However, demonstrating that these behaviors can be reduced or eliminated by knocking out MSLN, and that they are restored when survivin expression is rescued, would significantly advance the current understanding of the role of MSLN in carcinogenesis. 


\subsection{References}

1. Ai, J. et al. Nanotoxicology and nanoparticle safety in biomedical designs. Int. J. Nanomedicine 6, 1117-27 (2011).

2. Faraji, A. H. \& Wipf, P. Nanoparticles in cellular drug delivery. Bioorg. Med. Chem. 17, 2950-62 (2009).

3. Child, H. W. et al. Working together: the combined application of a magnetic field and penetratin for the delivery of magnetic nanoparticles to cells in 3D. ACS Nano 5, 7910-9 (2011).

4. Sam, S. et al. Semiquantitative study of the EDC/NHS activation of acid terminal groups at modified porous silicon surfaces. Langmuir 26, 809-14 (2010).

5. Thorek, D. L. J., Elias, D. R. \& Tsourkas, A. Comparative Analysis of NanoparticleAntibody Conjugations: Carbodiimide versus Click Chemistry. Mol. Imaging 8, 221229 (2009).

6. Wang, T.-H. \& Lee, W.-C. Immobilization of proteins on magnetic nanoparticles. Biotechnol. Bioprocess Eng. 8, 263-267 (2003).

7. Walsh, M. K., Wang, X. \& Weimer, B. C. Optimizing the immobilization of singlestranded DNA onto glass beads. J. Biochem. Biophys. Methods 47, 221-231 (2001).

8. Christiaens, P. et al. EDC-mediated DNA attachment to nanocrystalline CVD diamond films. Biosens. Bioelectron. 22, 170-177 (2006). 
9. Millan, K. M., Saraullo, a \& Mikkelsen, S. R. Voltammetric DNA biosensor for cystic fibrosis based on a modified carbon paste electrode. Anal. Chem. 66, 2943-2948 (1994).

10. Pelet, J. M. \& Putnam, D. An in-depth analysis of polymer-analogous conjugation using DMTMM. Bioconjug. Chem. 22, 329-37 (2011).

11. Smith, A. M. \& Nie, S. Compact Quantum Dots for Single-Molecule Imaging. J. Vis. Exp. August, 1-15 (2012).

12. Kunishima, M., Kawachi, C., Hioki, K., Terao, K. \& Tani, S. Formation of carboxamides by direct condensation of carboxylic acids and amines in alcohols using a new alcohol- and water-soluble condensing agent: DMT-MM. Tetrahedron 57, 1551-1558 (2001).

13. Thompson, K. \& Michielsen, S. Novel synthesis of N-substituted polyacrylamides: Derivatization of poly(acrylic acid) with amines using a triazine-based condensing reagent. J. Polym. Sci. Part A Polym. Chem. 44, 126-136 (2006).

14. Zhang, X., Mohandessi, S., Miller, L. W. \& Snee, P. T. Efficient functionalization of aqueous CdSe/ZnS nanocrystals using small-molecule chemical activators. Chem. Commun. (Camb). 47, 3532-4 (2011).

15. Conde, J. J. et al. Revisiting 30 years of biofunctionalization and surface chemistry of inorganic nanoparticles for nanomedicine. Front. Chem. 2, 1-27 (2014). 
16. Jin, R., Lin, B., Li, D. \& Ai, H. Superparamagnetic iron oxide nanoparticles for MR imaging and therapy: design considerations and clinical applications. Curr. Opin. Pharmacol. 18, 18-27 (2014).

17. Pisane, K., Despeaux, E. \& Seehra, M. S. Magnetic relaxation and correlating effective magnetic moment with particle size distribution in maghemite nanoparticles. J. Magn. Magn. Mater.

18. Mahmoudi, M., Hofmann, H., Rothen-Rutishauser, B. \& Petri-Fink, A. Assessing the in vitro and in vivo toxicity of superparamagnetic iron oxide nanoparticles. Chem. Rev. 112, 2323-38 (2012).

19. Marquis, B. J., Love, S. a, Braun, K. L. \& Haynes, C. L. Analytical methods to assess nanoparticle toxicity. Analyst 134, 425-39 (2009).

20. Laurent, S., Burtea, C., Thirifays, C., Häfeli, U. O. \& Mahmoudi, M. Crucial ignored parameters on nanotoxicology: the importance of toxicity assay modifications and "cell vision." PLoS One 7, e29997 (2012).

21. Fadeel, B. \& Garcia-Bennett, A. E. Better safe than sorry: Understanding the toxicological properties of inorganic nanoparticles manufactured for biomedical applications. Adv. Drug Deliv. Rev. 62, 362-74 (2010).

22. Gupta, A. K. \& Gupta, M. Cytotoxicity suppression and cellular uptake enhancement of surface modified magnetic nanoparticles. Biomaterials 26, 156573 (2005). 
23. Alexis, F., Pridgen, E., Molnar, L. K. \& Farokhzad, O. C. Factors Affecting the Clearance and Biodistribution of Polymeric Nanoparticles. Mol. Pharm. 5, 505-515 (2008).

24. Laurent, S. \& Mahmoudi, M. Superparamagnetic iron oxide nanoparticles: promises for diagnosis and treatment of cancer. Int. J. Mol. Epidemiol. Genet. 2, 367-90 (2011).

25. Cutler, J. I., Auyeung, E. \& Mirkin, C. a. Spherical nucleic acids. J. Am. Chem. Soc. 134, 1376-91 (2012).

26. Giljohann, D. a et al. Oligonucleotide loading determines cellular uptake of DNAmodified gold nanoparticles. Nano Lett. 7, 3818-21 (2007).

27. Hrkach, J. et al. Preclinical development and clinical translation of a PSMA-targeted docetaxel nanoparticle with a differentiated pharmacological profile. Sci. Transl. Med. 4, 128ra39 (2012).

28. Zhang, K., Hao, L., Hurst, S. J. \& Mirkin, C. a. Antibody-linked spherical nucleic acids for cellular targeting. J. Am. Chem. Soc. 134, 16488-91 (2012).

29. Mornet, S., Vasseur, S., Grasset, F. \& Duguet, E. Magnetic nanoparticle design for medical diagnosis and therapy. J. Mater. Chem. 14, 2161 (2004).

30. Lewinski, N., Colvin, V. \& Drezek, R. Cytotoxicity of nanoparticles. Small 4, 26-49 (2008). 
31. Mahmoudi, M., Laurent, S., Shokrgozar, M. a \& Hosseinkhani, M. Toxicity evaluations of superparamagnetic iron oxide nanoparticles: cell "vision" versus physicochemical properties of nanoparticles. ACS Nano 5, 7263-76 (2011).

32. Mikhaylova, M. et al. Superparamagnetism of magnetite nanoparticles: dependence on surface modification. Langmuir 20, 2472-7 (2004).

33. Yu, W. W., Chang, E., Sayes, C. M., Drezek, R. \& Colvin, V. L. Aqueous dispersion of monodisperse magnetic iron oxide nanocrystals through phase transfer. Nanotechnology 17, 4483-4487 (2006).

34. Tassa, C., Shaw, S. Y. \& Weissleder, R. Dextran-Coated Iron Oxide Nanoparticles: a Versatile Platform for Targeted Molecular Imaging, Molecular Diagnostics and Therapy. Acc Chem Res 44, 842-852 (2011).

35. Muldoon, L. L., S??ndor, M., Pinkston, K. E. \& Neuwelt, E. a. Imaging, Distribution, and Toxicity of Superparamagnetic Iron Oxide Magnetic Resonance Nanoparticles in the Rat Brain and Intracerebral Tumor. Neurosurgery 57, 785-796 (2005).

36. Shen, H., Jawaid, A. M. \& Snee, P. T. Poly (ethylene glycol) Carbodiimide Coupling Reagents for the Biological and Chemical Functionalization of Water- Soluble Nanoparticles. ACS Nano 3, 915-23 (2009).

37. Montalbetti, C. a. G. N. \& Falque, V. Amide bond formation and peptide coupling. Tetrahedron 61, 10827-10852 (2005). 
38. Wrobel, N., Schinkinger, M. \& Mirsky, V. M. A novel ultraviolet assay for testing side reactions of carbodiimides. Anal. Biochem. 305, 135-8 (2002).

39. Palazon, F. et al. Carbodiimide/NHS Derivatization of $\mathrm{COOH}-$ Terminated SAMs: Activation or Byproduct Formation? Langmuir 30, 4545-4550 (2014).

40. Kocbek, P., Obermajer, N., Cegnar, M., Kos, J. \& Kristl, J. Targeting cancer cells using PLGA nanoparticles surface modified with monoclonal antibody. J. Control. release 120, 18-26 (2007).

41. Natarajan, A. et al. NanoFerrite Particle Based Radioimmunonanoparticles: Binding affinity and in vivo pharmacokinetics. Bioconjug. Chem. 19, 1211-1218 (2008).

42. Booth, M. A., Kannappan, K., Hosseini, A. \& Partridge, A. In-Depth Electrochemical Investigation of Surface Attachment Chemistry via Carbodiimide Coupling. Langmuir 31, 8033-41 (2015).

43. Banerjee, A. et al. Fast, Efficient, and Stable Conjugation of Multiple DNA Strands on Colloidal Quantum Dots. Bioconjug. Chem. 26, 1582-9 (2015).

44. Tobiesen, F. A. \& Michielsen, S. Method for grafting poly(acrylic acid) onto nylon 6,6 using amine end groups on nylon surface. J. Polym. Sci. Part A Polym. Chem. 40, 719-728 (2002).

45. Wagner, K. et al. Synthesis of oligonucleotide-functionalized magnetic nanoparticles and study on their in vitro cell uptake. Appl. Organomet. Chem. 18, $514-519$ (2004). 
46. Santra, S., Kaittanis, C., Grimm, J. \& Perez, J. M. Drug/dye-Loaded, Multifunctional Iron Oxide Nanoparticles for Combined Targeted Cancer Therapy and Dual Optical/MR-Imaging. Small 5, 1862-8 (2009).

47. Abdollah, M. R. a et al. Prolonging the circulatory retention of SPIONs using dextran sulfate: in vivo tracking achieved by functionalisation with near-infrared dyes. Faraday Discuss. 175, 41-58 (2014).

48. Schneider, U. V et al. Increasing the analytical sensitivity by oligonucleotides modified with para- and ortho-twisted intercalating nucleic acids--TINA. PLoS One 6, e20565 (2011).

49. Wu, X. et al. Immunofluorescent labeling of cancer marker Her2 and other cellular targets with semiconductor quantum dots. Nat. Biotechnol. 21, 41-6 (2003).

50. $\mathrm{Bi}, \mathrm{X}$. et al. Gene Detection in Complex Biological Media Using Semiconductor Nanorods within an Integrated Microfluidic Device. Anal. Chem. 87, 10292-8 (2015).

51. Liu, L. et al. Activity analysis of the carbodiimide-mediated amine coupling reaction on self-assembled monolayers by cyclic voltammetry. Electrochim. Acta 89, 616622 (2013).

52. Holsapple, M. P. et al. Research strategies for safety evaluation of nanomaterials, part II: toxicological and safety evaluation of nanomaterials, current challenges and data needs. Toxicol. Sci. 88, 12-7 (2005). 
53. Warheit, D. B. How Meaningful are the Results of Nanotoxicity Studies in the Absence of Adequate Material Characterization? Toxicol. Sci. 101, 183-185 (2008).

54. Soenen, S. J., De Cuyper, M., De Smedt, S. C. \& Braeckmans, K. Investigating the toxic effects of iron oxide nanoparticles. Methods Enzymol. 509, 195-224 (Elsevier Inc., 2012).

55. Liu, Y., Zhao, Y., Sun, B. \& Chen, C. Understanding the Toxicity of Carbon Nanotubes. Acc. Chem. Res. 46, 702-713 (2013).

56. Heyder, J. Deposition of inhaled particles in the human respiratory tract and consequences for regional targeting in respiratory drug delivery. Proc. Am. Thorac. Soc. 1, 315-20 (2004).

57. Wahajuddin \& Arora, S. Superparamagnetic iron oxide nanoparticles: magnetic nanoplatforms as drug carriers. Int. J. Nanomedicine 7, 3445-71 (2012).

58. Love, S. a, Maurer-Jones, M. a, Thompson, J. W., Lin, Y.-S. \& Haynes, C. L. Assessing nanoparticle toxicity. Annu. Rev. Anal. Chem. (Palo Alto. Calif). 5, 181205 (2012).

59. Kroll, A., Pillukat, M. H., Hahn, D. \& Schnekenburger, J. Current in vitro methods in nanoparticle risk assessment: limitations and challenges. Eur. J. Pharm. Biopharm. 72, 370-7 (2009). 
60. Schulze, C. et al. Not ready to use - overcoming pitfalls when dispersing nanoparticles in physiological media. Nanotoxicology 2, 51-61 (2009).

61. Schweiger, C. et al. Quantification of the internalization patterns of superparamagnetic iron oxide nanoparticles with opposite charge. $\mathrm{J}$. Nanobiotechnology 10, 28 (2012).

62. Safi, M., Sarrouj, H., Sandre, O., Mignet, N. \& Berret, J.-F. Interactions between sub-10-nm iron and cerium oxide nanoparticles and 3 T3 fibroblasts: the role of the coating and aggregation state. Nanotechnology 21, 145103 (2010).

63. Li, S.-D. \& Huang, L. Targeted delivery of antisense oligodeoxynucleotide and small interference RNA into lung cancer cells. Mol. Pharm. 3, 579-88 (2006).

64. Kaaki, K. et al. Magnetic nanocarriers of doxorubicin coated with poly(ethylene glycol) and folic acid: relation between coating structure, surface properties, colloidal stability, and cancer cell targeting. Langmuir 28, 1496-505 (2012).

65. Omidirad, R., Rajabi Hosseinpour, F. \& Farahani, B. Preparation and in vitro drug delivery response of doxorubicin loaded PAA coated magnetite nanoparticles. J. Serbian Chem. Soc. 78, 1609-1616 (2013).

66. Mahajan, S., Koul, V., Choudhary, V., Shishodia, G. \& Bharti, A. C. Preparation and in vitro evaluation of folate-receptor-targeted SPION-polymer micelle hybrids for MRI contrast enhancement in cancer imaging. Nanotechnology 24, 015603 (2013). 
67. Shi, J., Xiao, Z., Kamaly, N. \& Farokhzad, O. C. Self-assembled targeted nanoparticles: evolution of technologies and bench to bedside translation. Acc. Chem. Res. 44, 1123-34 (2011).

68. Gangwal, S. et al. Informing Selection of Nanomaterial Concentrations for ToxCast in Vitro Testing Based on Occupational Exposure Potential. Environ. Health Perspect. 119, 1539-1547 (2011).

69. Hyeon, T., Lee, S. S., Park, J., Chung, Y. \& Na, H. B. Synthesis of highly crystalline and monodisperse maghemite nanocrystallites without a size-selection process. J. Am. Chem. Soc. 123, 12798-801 (2001).

70. Lundqvist, M. et al. Nanoparticle size and surface properties determine the protein corona with possible implications for biological impacts. Proc. Natl. Acad. Sci. U. S. A. 105, 14265-70 (2008).

71. Troutier, A.-L., Véron, L., Delair, T., Pichot, C. \& Ladavière, C. New insights into self-organization of a model lipid mixture and quantification of its adsorption on spherical polymer particles. Langmuir 21, 9901-10 (2005).

72. Qin, J. et al. A High-Performance Magnetic Resonance ImagingT2 Contrast Agent. Adv. Mater. 19, 1874-1878 (2007).

73. Petri-Fink, A., Steitz, B., Finka, A., Salaklang, J. \& Hofmann, H. Effect of cell media on polymer coated superparamagnetic iron oxide nanoparticles (SPIONs): colloidal 
stability, cytotoxicity, and cellular uptake studies. Eur. J. Pharm. Biopharm. 68, 129-37 (2008).

74. Gonzales, M., Misumori, L. M., Kushleika, J. V., Rosenfeld, M. E. \& Krishnan, K. M. Cytotoxicity of iron oxide nanoparticles made from the thermal decomposition of organometallics and aqueous phase transfer with Pluronic F127. Contrast Media Mol. Imaging 5, 286-293 (2011).

75. Thode, K., Luck, M., Semmler, W., Muller, R. H. \& Kresse, M. Determination of Plasma Protein Adsorption on Magnetic Iron Oxide_Sample Preparation.pdf. Pharm. Res. 14, 905-910 (1997).

76. Kumar, M. et al. Cellular interaction of folic acid conjugated superparamagnetic iron oxide nanoparticles and its use as contrast agent for targeted magnetic imaging of tumor cells. Int. J. Nanomedicine 7, 3503-16 (2012).

77. Chang, S. Y. et al. Analysis of peptides and proteins affinity-bound to iron oxide nanoparticles by MALDI MS. J. Am. Soc. Mass Spectrom. 18, 910-8 (2007).

78. Kotek, G. et al. Cell quantification: evolution of compartmentalization and distribution of iron-oxide particles and labeled cells. Contrast Media Mol. Imaging 7, 195-203 (2012).

79. Huang, J., Zhong, X., Wang, L., Yang, L. \& Mao, H. Improving the magnetic resonance imaging contrast and detection methods with engineered magnetic nanoparticles. Theranostics 2, 86-102 (2012). 
80. Wu, Y. J. et al. In vivo leukocyte labeling with intravenous ferumoxides / protamine sulfate complex and in vitro characterization for cellular magnetic resonance imaging. Am. J. Physiol. Cell Physiol. 293, C1698-C1708 (2007).

81. Boutry, S. et al. How to quantify iron in an aqueous or biological matrix: a technical note. Contrast Media Mol. Imaging 4, 299-304 (2009).

82. Boutry, S. et al. Magnetic labeling of non-phagocytic adherent cells with iron oxide nanoparticles: a comprehensive study. Contrast Media Mol. Imaging 3, 223-32 (2008).

83. Riemer, J., Hoepken, H. H., Czerwinska, H., Robinson, S. R. \& Dringen, R. Colorimetric ferrozine-based assay for the quantitation of iron in cultured cells. Anal. Biochem. 331, 370-5 (2004).

84. Fan, C. et al. Tumor selectivity of stealth multi-functionalized superparamagnetic iron oxide nanoparticles. Int. J. Pharm. 404, 180-90 (2011).

85. Kalambur, V. S., Longmire, E. K. \& Bischof, J. C. Cellular level loading and heating of superparamagnetic iron oxide nanoparticles. Langmuir 23, 12329-36 (2007).

86. Olie, R. A., Simões-wüst, A. P., Baumann, B. \& Simo, A. P. A Novel Antisense Oligonucleotide Targeting Survivin Expression Induces Apoptosis and Sensitizes Lung Cancer Cells to Chemotherapy. Cancer Res. 60, 2805-2809 (2000).

87. Dias, N. \& Stein, C. A. Antisense Oligonucleotides: Basic Concepts and Mechanisms. Mol. Cancer Ther. 1, 347-355 (2002). 
88. Carter, J. D. \& Labean, T. H. Organization of Inorganic Nanomaterials via Programmable DNA Self-Assembly and Peptide Molecular Recognition. ACS Nano 5, 2200-2205 (2012).

89. Shukoor, M. I. et al. dsRNA-functionalized multifunctional gamma-Fe2O3 nanocrystals: a tool for targeting cell surface receptors. Angew. Chem. Int. Ed. Engl. 47, 4748-52 (2008).

90. Patel, P. C. et al. Scavenger Receptors Mediate Cellular Uptake of Polyvalent Oligonucleotide-Functionalized Gold Nanoparticles. Bioconjug. Chem. 21, 22502256 (2010).

91. Farokhzad, O. C. et al. Nanoparticle-aptamer bioconjugates: a new approach for targeting prostate cancer cells. Cancer Res. 64, 7668-72 (2004).

92. Reddy, L. H., Arias, J. L., Nicolas, J. \& Couvreur, P. Magnetic nanoparticles: design and characterization, toxicity and biocompatibility, pharmaceutical and biomedical applications. Chem. Rev. 112, 5818-78 (2012).

93. Caballero-Díaz, E. \& Valcárcel Cases, M. Analytical methodologies for nanotoxicity assessment. Trends Anal. Chem. (2016). doi:10.1016/j.trac.2016.03.007

94. Tamm, I. et al. IAP-Family Protein Survivin Inhibits Caspase Activity and Apoptosis Induced by Fas (CD95), Bax, Caspases, and Anticancer Drugs. Cancer Res. 58, 5315-5320 (1998). 
95. Nakahara, T. et al. Broad spectrum and potent antitumor activities of YM155, a novel small-molecule survivin suppressant, in a wide variety of human cancer cell lines and xenograft models. Cancer Sci. 102, 614-21 (2011).

96. Jarzynka, M. J., Guo, P., Bar-Joseph, I., Hu, B. \& Cheng, S.-Y. Estradiol and nicotine exposure enhances A549 bronchioloalveolar carcinoma xenograft growth in mice through the stimulation of angiogenesis. Int. J. Oncol. 28, 337-44 (2006).

97. Wang, W. et al. Anti-lung cancer effects of novel ginsenoside 25-OCH(3)-PPD. Lung Cancer 65, 306-11 (2009).

98. Altieri, D. C. The molecular basis and potential role of survivin in cancer diagnosis and therapy. Trends Mol. Med. 7, 542-7 (2001).

99. Monteiro-Riviere, N. a, Inman, a O. \& Zhang, L. W. Limitations and relative utility of screening assays to assess engineered nanoparticle toxicity in a human cell line. Toxicol. Appl. Pharmacol. 234, 222-35 (2009).

100. Smith, S. M., Wunder, M. B., Norris, D. a \& Shellman, Y. G. A simple protocol for using a LDH-based cytotoxicity assay to assess the effects of death and growth inhibition at the same time. PLoS One 6, e26908 (2011).

101. Li, F. Survivin study: what is the next wave? J. Cell. Physiol. 197, 8-29 (2003).

102. Carter, B. Z., Wang, R., Schober, W. D., Milella, M. \& Andreeff, M. Targeting Survivin Expression Induces Cell Proliferation Defect and Subsequent Cell Death 
Involving Mitochondrial Pathway in Myeloid Leukemic Cell. Cell Cycle 2, 488-493 (2003).

103. Cong, X. L. \& Han, Z. C. Survivin and Leukemia. Int. J. Hematol. 80, 232-238 (2004).

104. Carrasco, R. a et al. Antisense inhibition of survivin expression as a cancer therapeutic. Mol. Cancer Ther. 10, 221-32 (2011).

105. Rivadeneira, D. B. et al. Survivin promotes oxidative phosphorylation, subcellular mitochondrial repositioning, and tumor cell invasion. Sci. Signal. 8, ra80 (2015).

106. Li, H., Niederkorn, J. Y., Neelam, S. \& Alizadeh, H. Downregulation of survivin expression enhances sensitivity of cultured uveal melanoma cells to cisplatin treatment. Exp. Eye Res. 83, 176-82 (2006).

107. Liu, W.-S. et al. siRNA directed against survivin enhances pancreatic cancer cell gemcitabine chemosensitivity. Dig. Dis. Sci. 54, 89-96 (2009).

108. Altieri, D. C. Validating survivin as a cancer therapeutic target. Nat. Rev. Cancer 3, $46-54$ (2003).

109. Mita, A. C., Mita, M. M., Nawrocki, S. T. \& Giles, F. J. Survivin: key regulator of mitosis and apoptosis and novel target for cancer therapeutics. Clin. Cancer Res. $14,5000-5(2008)$. 
110. Sasaki, T., Lopes, M. B. S., Hankins, G. R. \& Helm, G. a. Expression of survivin, an inhibitor of apoptosis protein, in tumors of the nervous system. Acta Neuropathol. 104, 105-9 (2002).

111. Zhang, M., Latham, D. E., Delaney, M. a \& Chakravarti, A. Survivin mediates resistance to antiandrogen therapy in prostate cancer. Oncogene 24, 2474-82 (2005).

112. Osaka, E. et al. Survivin as a prognostic factor for osteosarcoma patients. Acta Histochem. Cytochem. 39, 95-100 (2006).

113. Zamparese, R. et al. Survivin expression in renal cell carcinoma. Cancer Invest. 26, 929-35 (2008).

114. Kawasaki, H., Altieri, D. C. \& Lu, C. Inhibition of Apoptosis by Survivin Predicts Shorter Survival Rates in Colorectal Cancer. Cancer Res. 58, 5071-5074 (1998).

115. Hansen, J. B. et al. SPC3042: a proapoptotic survivin inhibitor. Mol. Cancer Ther. 7, 2736-45 (2008).

116. Wobser, M. et al. Complete remission of liver metastasis of pancreatic cancer under vaccination with a HLA-A2 restricted peptide derived from the universal tumor antigen survivin. Cancer Immunol. Immunother. 55, 1294-8 (2006).

117. Yu, M. K. et al. Drug-loaded superparamagnetic iron oxide nanoparticles for combined cancer imaging and therapy in vivo. Angew. Chem. Int. Ed. Engl. 47, $5362-5(2008)$. 
118. Zhu, L. et al. Multifunctional pH-sensitive superparamagnetic iron-oxide nanocomposites for targeted drug delivery and MR imaging. J. Control. Release $169,228-38$ (2013).

119. Akbarzadeh, A. et al. Preparation and in vitro evaluation of doxorubicin-loaded $\mathrm{Fe}_{3} \mathrm{O}_{4}$ magnetic nanoparticles modified with biocompatible copolymers. Int. J. Nanomedicine 7, 511-26 (2012).

120. Wilson, M. W. et al. Hepatocellular carcinoma: regional therapy with a magnetic targeted carrier bound to doxorubicin in a dual MR imaging/ conventional angiography suite--initial experience with four patients. Radiology 230, 287-93 (2004).

121. Munnier, E. et al. Novel method of doxorubicin-SPION reversible association for magnetic drug targeting. Int. J. Pharm. 363, 170-6 (2008).

122. Fuessel, S. et al. Chemosensitization of bladder cancer cells by survivin-directed antisense oligodeoxynucleotides and siRNA. Cancer Lett. 232, 243-54 (2006).

123. Zaffaroni, N. et al. Expression of the anti-apoptotic gene survivin correlates with taxol resistance in human ovarian cancer. Cell. Mol. Life Sci. 59, 1406-12 (2002).

124. Moriai, R., Tsuji, N., Moriai, M., Kobayashi, D. \& Watanabe, N. Survivin plays as a resistant factor against tamoxifen-induced apoptosis in human breast cancer cells. Breast Cancer Res. Treat. 117, 261-71 (2009). 
125. Barr, M. P. et al. Generation and characterisation of cisplatin-resistant non-small cell lung cancer cell lines displaying a stem-like signature. PLoS One 8, e54193 (2013).

126. Jain, T. K., Reddy, M. K., Morales, M. A., Leslie-pelecky, D. L. \& Labhasetwar, V. Biodistribution, Clearance, and Biocompatibility of Iron Oxide Magnetic Nanoparticles in Rats. Mol. Pharm. 5, 316-327 (2008).

127. Maurizi, L., Sakulkhu, U., Gramoun, A., Vallee, J.-P. \& Hofmann, H. A fast and reproducible method to quantify magnetic nanoparticle biodistribution. Analyst 139, 1184-91 (2014).

128. Schlachter, E. K. et al. Metabolic pathway and distribution of superparamagnetic iron oxide nanoparticles: in vivo study. Int. J. Nanomedicine 6, 1793-800 (2011).

129. Hoebeke, J. et al. Design of Folic Acid-Conjugated Nanoparticles for Drug Targeting. J. Pharm. Sci. 89, 1452-1464 (2000).

130. Kim, S. H., Jeong, J. H., Chun, K. W. \& Park, T. G. Target-specific cellular uptake of PLGA nanoparticles coated with poly(L-lysine)-poly(ethylene glycol)-folate conjugate. Langmuir 21, 8852-7 (2005).

131. Dey, S. \& Maiti, T. K. Superparamagnetic Nanoparticles and RNAi-Mediated Gene Silencing: Evolving Class of Cancer Diagnostics and Therapeutics. J. Nanomater. 2012, 1-15 (2012). 
132. Mahmoudi, M., Sant, S., Wang, B., Laurent, S. \& Sen, T. Superparamagnetic iron oxide nanoparticles (SPIONs): development, surface modification and applications in chemotherapy. Adv. Drug Deliv. Rev. 63, 24-46 (2011).

133. Reker, S. et al. Identification of Novel Survivin-Derived CTL Epitopes. Cancer Biol. Ther. 3, 173-179 (2004).

134. Andersen, M. H. et al. Spontaneous Cytotoxic T-Cell Responses against Survivinderived MHC Class I-restricted T-Cell Epitopes in Situ As Well As ex Vivo in Cancer Patients Advances in Brief As Well As ex Vivo in Cancer Patients 1. Cancer Res. 61, 5964-5968 (2001).

135. Shamah, S. M., Healy, J. M. \& Cload, S. T. Complex target SELEX. Acc. Chem. Res. 41, 130-8 (2008).

136. Bachinsky, M. M. et al. Mapping and binding analysis of peptides derived from the tumor-associated antigen survivin for eight HLA alleles. Cancer Immun. 5, 6-15 (2005).

137. Case, D. A. et al. Amber 12. (2012).

138. Chushak, Y. \& Stone, M. O. In silico selection of RNA aptamers. Nucleic Acids Res. 37, e87 (2009).

139. Morris, G. M. et al. AutoDock4 and AutoDockTools4: Automated Docking with Selective Receptor Flexibility. J. Comput. Chem. 30, 2785-2791 (2009). 
140. Di Primo, C. \& Lebars, I. Determination of refractive index increment ratios for protein-nucleic acid complexes by surface plasmon resonance. Anal. Biochem. 368, 148-55 (2007).

141. Shukla, A., Vacek, P. \& Mossman, B. T. Dose-Response Relationships in Expression of Biomarkers of Cell Proliferation in In Vitro Assays and Inhalation Experiments. Nonlinearity Biol. Toxicol. Med. 2, 117-28 (2004).

142. Wang, L. et al. Neoplastic-like transformation effect of single-walled and multiwalled carbon nanotubes compared to asbestos on human lung small airway epithelial cells. Nanotoxicology 8, 485-507 (2014).

143. Wang, L. et al. Carbon Nanotubes Induce Malignant Transformation and Tumorigenesis of Human Lung Epithelial Cells. Nano Lett. 11, 2796-2803 (2011).

144. Lohcharoenkal, W. et al. Chronic exposure to carbon nanotubes induces invasion of human mesothelial cells through matrix metalloproteinase-2. ACS Nano 7, 7711$23(2013)$.

145. Stueckle, T. a et al. Chronic occupational exposure to arsenic induces carcinogenic gene signaling networks and neoplastic transformation in human lung epithelial cells. Toxicol. Appl. Pharmacol. 261, 204-16 (2012).

146. Luanpitpong, S., Wang, L., Castranova, V. \& Rojanasakul, Y. Induction of stem-like cells with malignant properties by chronic exposure of human lung epithelial cells to single-walled carbon nanotubes. Part. Fibre Toxicol. 11, 22 (2014). 
147. Pongrakhananon, V. et al. Carbon nanotubes induce apoptosis resistance of human lung epithelial cells through FLICE-inhibitory protein. Toxicol. Sci. 143, 499511 (2015).

148. Kachala, S. S. et al. Mesothelin overexpression is a marker of tumor aggressiveness and is associated with reduced recurrence-free and overall survival in early-stage lung adenocarcinoma. Clin. cancer Res. 20, 1020-8 (2014).

149. Tang, Z., Qian, M. \& Ho, M. The role of mesothelin in tumor progression and targeted therapy. Anticancer Agents Med Chem 13, 276-280 (2013).

150. Chen, D. et al. Gene expression profile of human lung epithelial cells chronically exposed to single-walled carbon nanotubes. Nanoscale Res. Lett. 10, (2015).

151. Bharadwaj, U., Li, M., Chen, C. \& Yao, Q. Mesothelin-induced pancreatic cancer cell proliferation involves alteration of cyclin E via activation of signal transducer and activator of transcription protein 3. Mol. Cancer Res. 6, 1755-65 (2008).

152. Chang, M.-C. et al. Mesothelin enhances invasion of ovarian cancer by inducing MMP-7 through MAPK/ERK and JNK pathways. Biochem. J. 442, 293-302 (2012).

153. Servais, E. L. et al. Mesothelin overexpression promotes mesothelioma cell invasion and MMP-9 secretion in an orthotopic mouse model and in epithelioid pleural mesothelioma patients. Clin. cancer Res. 18, 2478-89 (2012). 
154. Bharadwaj, U., Marin-Muller, C., Li, M., Chen, C. \& Yao, Q. Mesothelin overexpression promotes autocrine IL-6/sIL-6R trans-signaling to stimulate pancreatic cancer cell proliferation. Carcinogenesis 32, 1013-24 (2011).

155. Zheng, C. et al. Mesothelin regulates growth and apoptosis in pancreatic cancer cells through p53-dependent and-independent signal pathway. J. Exp. Clin. Cancer Res. 31, 84-98 (2012).

156. Luanpitpong, S. et al. Caveolin-1 regulates lung cancer stem-like cell induction and p53 inactivation in carbon nanotube-driven tumorigenesis. Oncotarget 5, 35413554 (2014).

157. Alvarez, H. et al. Mesothelin is a specific biomarker of invasive cancer in the Barrettassociated adenocarcinoma progression model: translational implications for diagnosis and therapy. Nanomedicine 4, 295-301 (2008).

158. $\mathrm{Li}, \mathrm{M}$. et al. Mesothelin is a malignant factor and therapeutic vaccine target for pancreatic cancer. Mol. Cancer Ther. 7, 286-96 (2008).

159. Gritsko, T. et al. Persistent activation of stat3 signaling induces survivin gene expression and confers resistance to apoptosis in human breast cancer cells. Clin. cancer Res. 12, 11-9 (2006).

160. Chen, X., Duan, N., Zhang, C. \& Zhang, W. Survivin and Tumorigenesis: Molecular Mechanisms and Therapeutic Strategies. J. Cancer 7, 314-23 (2016). 
161. Aoki, Y., Feldman, G. M. \& Tosato, G. Inhibition of STAT3 signaling induces apoptosis and decreases survivin expression in primary effusion lymphoma. Blood 101, 1535-1542 (2003).

162. Diaz, N. et al. Activation of stat3 in primary tumors from high-risk breast cancer patients is associated with elevated levels of activated SRC and survivin expression. Clin. cancer Res. 12, 20-8 (2006).

163. Melaiu, O. et al. MSLN gene silencing has an anti-malignant effect on cell lines overexpressing mesothelin deriving from malignant pleural mesothelioma. PLoS One 9, e85935 (2014).

164. Gao, F. et al. Survivin promotes the invasion of human colon carcinoma cells by regulating the expression of MMP-7. Mol. Med. Rep. 9, 825-30 (2014).

165. Bharadwaj, U., Marin-Muller, C., Li, M., Chen, C. \& Yao, Q. Mesothelin confers pancreatic cancer cell resistance to TNF- $\alpha$-induced apoptosis through Akt/PI3K/NF-kB activation and IL-6/Mcl-1 overexpression. Mol. Cancer 10, 106 (2011).

166. Kanwar, J. R., Kamalapuram, S. K. \& Kanwar, R. K. Survivin Signaling in Clinical Oncology: A Multifaceted Dragon. Med. Res. Rev. 765-789 (2012). doi:10.1002/med 
167. Hoffman, W. H., Biade, S., Zilfou, J. T., Chen, J. \& Murphy, M. Transcriptional repression of the anti-apoptotic survivin gene by wild type p53. J. Biol. Chem. 277, 3247-57 (2002).

168. Kanda, N. et al. STAT3 is constitutively activated and supports cell survival in association with survivin expression in gastric cancer cells. Oncogene 23, 4921-9 (2004). 